\title{
Developments for improved diagnosis of bloodstream infections
}

Citation for published version (APA):

Loonen, A. J. M. (2013). Developments for improved diagnosis of bloodstream infections. [Doctoral Thesis, Maastricht University]. Maastricht University. https://doi.org/10.26481/dis.20131217al

Document status and date:

Published: 01/01/2013

DOI:

10.26481/dis.20131217al

Document Version:

Publisher's PDF, also known as Version of record

\section{Please check the document version of this publication:}

- A submitted manuscript is the version of the article upon submission and before peer-review. There can be important differences between the submitted version and the official published version of record.

People interested in the research are advised to contact the author for the final version of the publication, or visit the DOI to the publisher's website.

- The final author version and the galley proof are versions of the publication after peer review.

- The final published version features the final layout of the paper including the volume, issue and page numbers.

Link to publication

\footnotetext{
General rights rights.

- You may freely distribute the URL identifying the publication in the public portal. please follow below link for the End User Agreement:

www.umlib.nl/taverne-license

Take down policy

If you believe that this document breaches copyright please contact us at:

repository@maastrichtuniversity.nl

providing details and we will investigate your claim.
}

Copyright and moral rights for the publications made accessible in the public portal are retained by the authors and/or other copyright owners and it is a condition of accessing publications that users recognise and abide by the legal requirements associated with these

- Users may download and print one copy of any publication from the public portal for the purpose of private study or research.

- You may not further distribute the material or use it for any profit-making activity or commercial gain

If the publication is distributed under the terms of Article $25 \mathrm{fa}$ of the Dutch Copyright Act, indicated by the "Taverne" license above, 
Developments for improved diagnosis of bloodstream infections 
(C) A.J.M. Loonen, 2013.

All rights reserved. No part of this thesis may be reproduced or transmitted in any form or by any means, electronic or mechanical, including photocopy, without prior permission of the publisher and copyright owner, or where appropriate, the publisher of the articles.

ISBN/EAN: $\quad 978-94-6108-546-7$

Cover design: Annelies Santegoets

Lay-out: $\quad$ Gildeprint, Enschede

Printed by: Gildeprint, Enschede 


\title{
Developments for improved diagnosis of bloodstream infections
}

\author{
PROEFSCHRIFT \\ Ter verkrijging van de graad van doctor aan de Universiteit Maastricht, \\ op gezag van de Rector Magnificus Prof. dr. L.L.G. Soete \\ volgens het besluit van het College van Decanen, \\ in het openbaar te verdedigen op dinsdag 17 december 2013 om 16.00 uur
}

door

Anna Johanna Maria Loonen geboren te Goirle op 18 januari 1983 


\section{Promotor}

Prof. dr. C.A. Bruggeman

\section{Copromotores}

Dr. A.J.C. van den Brule (Jeroen Bosch Ziekenhuis)

Dr. ir. P.F.G. Wolffs

\section{Beoordelingscommissie}

Prof. dr. E.J.M. Speel (voorzitter)

Dr. D.C.J.J. Bergmans

Dr. W.B. van Leeuwen (Erasmus Medisch Centrum)

Prof. dr. H.J.M. Smeets

Prof. dr. L.J.I. Zimmermann

Financial support for the publication of this thesis was kindly provided by:

- Fontys Hogeschool Toegepaste Natuurwetenschappen

- Jeroen Bosch Academy

- Stichting PAMM

- Gambro Lundia AB

- Biocartis N.V.

- Roche Diagnostics Nederland B.V. 
When things go wrong as they sometimes will When the road you're trudging seems all up hill.

Don't give up though the pace seems slow You may succeed with another blow.

Success is failure turned inside out The silver tint of the clouds of doubt

And you never can tell how close you are It may be near when it seems so far.

So stick to the fight when you're hardest hit It's when things seem worst that you must not quit.

Author unknown 



\section{Table of contents}

Chapter 1 General Introduction and Outline of the Thesis 9

Chapter 2 Development and clinical evaluation of a tuf gene based realtime PCR for Gram positive cocci: a study using blood cultures and whole blood

Chapter 3 Acceleration of direct identification of Staphylococcus aureus versus coagulase-negative staphylococci from blood culture material: a comparison of six bacterial DNA extraction methods

Chapter 4 Comparative study using phenotypic, genotypic and proteomics methods for identification of coagulase-negative staphylococci

Chapter 5 Speciation of coagulase-negative staphylococci from positive blood cultures using MALDI-TOF MS: clinical relevance

Chapter 6 An evaluation of three processing methods and the effect of reduced culture times for faster direct identification of pathogens from BacT/ALERT blood cultures by MALDI-TOF MS

Chapter 7 Comparison of pathogen DNA isolation methods from large volumes of whole blood to improve molecular diagnosis of bloodstream infections

Chapter 8 Biomarkers and molecular analysis to improve bloodstream infection diagnostics in an emergency care unit

Chapter 9 Tuf mRNA might be a promising marker to detect viable Staphylococcus aureus in blood of bacteremic patients

Chapter 10 Summary, General Discussion and Future Perspectives

Chapter 11 Samenvatting, Discussie en Toekomstvisies 



\section{Chapter 1}

\section{General Introduction and Outline of the Thesis}




\section{Preface}

Bloodstream infections (BSIs) are characterized by high morbidity and mortality all over the world. BSI can be categorized as either community acquired or hospital acquired and can be the result of a broad variety of microorganisms. Both bacteria and fungi can enter the, normal sterile, bloodstream. These conditions are called bacteremia and fungemia, respectively. The first case of bacteremia was already reported in 1897 by Libman [1]. Since then, culture methods for blood have evolved, with the development of guidelines for optimal handling of blood cultures [2]. Currently, blood cultures are still the gold standard, for detection of pathogens from blood, in the medical microbiology laboratories. The possibility to detect and identify the pathogen, and simultaneously determine the antimicrobial susceptibility pattern is very helpful to guide physicians to optimal patient-tailored antibiotic therapy. However, this traditional approach for detection of pathogens from blood is time-consuming (minimal 24-72 hours), and prior antibiotic treatment of a patient can result in false-negative blood cultures. New developments to detect bloodstream infections (BSI) are needed as it has been shown by Kumar et al. [3] that initiation of inappropriate antibiotic therapy decreases the survival rate of patients fivefold. However, misuse of antibiotics increases the emergence of antibiotic resistance among bacteria, which in turn hampers further antibiotic treatment [4]. This means that other techniques, besides conventional culture approaches, need to be developed, and used, to improve the diagnostic process of detection of bloodstream infections to be able to initiate adequate therapy.

In the present chapter, an overview will be given of bloodstream infections, and the currently available diagnostic tools in the laboratories for molecular diagnostics and medical microbiology.

\section{Bloodstream infections}

BSI can be caused by a broad scala of microorganisms, including bacteria (bacteremia) and fungi (fungemia). It is defined as the presence of viable microorganisms in the bloodstream [5]. BSI is often a result of infection due to trauma, use of intravascular devices, invasive procedures (surgery), or a result of infection at another organ site (i.e. respiratory tract infections) [6]. The host response to (systemic) infection, such as BSI, can lead to Systemic Inflammatory Response Syndrome (SIRS). The term SIRS was introduced in 1992 at the American College of Chest Physicians/Society of Critical Care Medicine (ACCP/SCCM) Consensus Conference and was used to describe a systemic inflammatory process, despite of its cause. Four clinical symptoms can be recognised and are indicative of SIRS, as summarized in Table 1 [7]. 
Table 1. Systemic Inflammatory Response Syndrome (SIRS) and sepsis stages.

\begin{tabular}{|c|c|c|}
\hline Stage & Clinical symptoms & Value \\
\hline SIRS & Temperature & $<36^{\circ} \mathrm{C}$ or $>38^{\circ} \mathrm{C}$ \\
\hline \multirow[t]{3}{*}{ ( $\geq 2$ symptoms) } & Heart rate & $>90$ beats/minute \\
\hline & Respiratory rate & $>20$ breaths/minute or $\mathrm{PaCO}_{2}<32 \mathrm{mmHg}$ \\
\hline & White blood cell count & $>12,000$ cells $/ \mathrm{mm}^{3}$ or $<$ than 4000 cells $/ \mathrm{mm}^{3}$ \\
\hline Sepsis & SIRS with proven infection & \\
\hline \multirow[t]{2}{*}{ Severe sepsis } & Sepsis with organ dysfunction & \\
\hline & hypoperfusion and/or hypotension & \\
\hline \multirow[t]{3}{*}{ Septic shock } & Severe sepsis, hypoperfusion & \\
\hline & and/or hypotension despite & \\
\hline & adequate fluid resuscitation & \\
\hline
\end{tabular}

A patient is diagnosed with sepsis when $\geq 2$ SIRS symptoms are present in combination with a proven infection. Sepsis has different severity stages and can develop to severe sepsis and septic shock (Table 1). Frequently, infection is strongly suspected but not microbiologically confirmed [8]. In other words, blood cultures drawn from a suspected sepsis patient can be negative.

Sepsis ranks in the top 10 causes of death [9]. The prevalence of sepsis in intensive care units (ICU) remains increasing [10]. A Dutch study performed in 2004 by Van Gestel et al. [11] calculated that the annual number of admissions for severe sepsis in Dutch ICUs was $8643+/-929$ cases/year, which is $0,054 \%$ of the total population, $0,61 \%$ of hospital admissions and $11 \%$ of ICU admissions. On average 750,000 patients develop sepsis in the United States annually [12]. The mortality rate ranges from 20 to $70 \%$ and is related to severity of underlying illness [13-16]. In Germany, approximately 79,000 new sepsis cases, and 75,000 severe sepsis cases are annually expected [17].

The accurate and timely detection of sepsis remains a challenge. The main problem is that the clinical signs of a patient with sepsis resemble that of a patient with SIRS caused by non-infectious causes, such as pancreatitis or burns [7]. Accurate and fast identification of pathogens involved and biomarkers might be of additional value in the diagnosis of BSI in suspected sepsis patients.

\section{$2.1 \quad$ Biomarkers}

In general, a biomarker has been defined as the "quantifiable measurement(s) of biological homeostasis that define(s) what is normal; therefore providing a frame of reference for predicting or detecting what is abnormal" [18]. It is highly unlikely that one single biomarker is able to predict BSI in sepsis patients. 
Several studies have been published which investigated the diagnostic value of several biomarkers, such as C-reactive protein (CRP) [19-21], procalcitonin (PCT) [19-22], neutrophil lymphocyte-count-ratio (NLCR) [23], and soluble urokinase plasminogen activator receptor (suPAR) [24-28].

$\mathrm{CRP}$ is an acute-phase protein released by the liver after the onset of inflammation or tissue damage. CRP is a frequently used marker to detect infection and inflammation. It is cheap, but not sufficiently specific for an accurate diagnosis of severe sepsis [19, 21]. Reasons for this might be that concentrations of CRP also increase during minor infections and therefore do not adequately predict the severity of the infection. Additionally, plasma levels remain elevated for several days, even when the infection is treated [29]. There has been a lot of debate about the usefulness of CRP in determining the prognosis of sepsis [30], and a more promising candidate might be PCT.

PCT is the prohormone of calcitonin, and is produced ubiquitously in response to endotoxin or to mediators released in response to bacterial infections (i.e. Interleukin 6). It strongly correlates with the extent and severity of bacterial infections [31]. Several meta-analyses studies have been published but results remain contradictory [32-34]. This is mainly the result of different patient inclusion criteria. However, in most studies PCT appears superior to CRP in differentiating and predicting severe sepsis patients from other patients [21, 35].

As the physiological immune response of circulating leucocytes to various stressful events is often characterized by an increase in neutrophil counts and a decline in lymphocyte counts, Zahorec et al. proposed to use the ratio of both as an additional infection marker in clinical ICU practice [36]. This so-called neutrophil-lymphocyte stress factor was found to correlate well with the severity of disease and outcome. De Jager et al. have investigated the ratio of neutrophil and lymphocyte counts, referred to as the NLCR, in predicting bacteremia in an emergency care unit [23]. They showed that this marker has a high value in predicting bacteremia, is simple, easily obtained and calculated, easy to integrate in daily practice and without extra costs. It would be very interesting to investigate if NLCR has more value in daily practice to predict $\mathrm{BSI}$ in sepsis patients as compared to i.e. CRP, which is determined standard in most hospitals in The Netherlands.

The urokinase-type plasminogen activator system consists of a proteinase (UPA), a receptor (UPAR) and inhibitors. suPAR is the soluble form of UPAR. In 1990, UPAR was cloned [37], and, in 1991 the soluble form was discovered [38]. The system plays a role in various immunological functions, including cell adhesion, migration, chemotaxis, proteolysis, immune activation, invasion, and signal transduction [39]. UPA and UPAR are predominantly expressed by tumor cells and blood cells, including neutrophils, monocytes, macrophages, and activated T-cells [40]. After cleavage from the cell surface, suPAR can be found in the blood and other body fluids in all individuals. Elevated suPAR levels, in septic intensive care unit patients, were already reported in 1995 by Mizukami et al. [41]. Koch et al. published one 
of the first studies in which the diagnostic and prognostic impact of suPAR was investigated in a large cohort of critically ill patients [28]. They found that suPAR serum concentrations, in sepsis and non-sepsis patients, are increased upon admission to the intensive care unit, probably reflecting the state of the immune system. These levels remained stably elevated in the initial course of treatment. Low suPAR levels were reported to be a positive predictor of overall survival in critically ill patients, including sepsis patients [26, 28]. Especially in the emergency department, it is crucial to categorize individual patients according to their disease severity and determine treatment priority. In the heterogeneous patient population which presents at the emergency department, suPAR might be very valuable to determine who needs intensive monitoring [42]. More clinical studies are needed to determine suPAR cut-off levels for optimal clinical management. Moreover, the association of biomarkers needs to be further addressed in different patient populations such as the intensive care and the emergency care unit.

\subsection{Etiologic agents of BSI and clinical load}

BSI can be the result of a broad scala of pathogens. One of the largest multicenter studies found that hospital acquired (nosocomial) BSI is in $65 \%$ of the cases a result of Gram positive bacteria, in $25 \%$ Gram negative bacteria are found, and $10 \%$ of BSI cases are caused by fungi [43]. The most commonly isolated pathogens were coagulase-negative staphylococci (31\%), Staphylococcus aureus (20\%), and Enterococcus spp. (9\%) [43]. Gram-negative bacteria that are frequently detected from blood cultures are Escherichia coli $(27 \%)$, Pseudomonas aeruginosa (15\%), and Klebsiella pneumoniae (7\%) [44]. Nosocomial fungal infections are mostly the result of Candida spp., and are associated with high morbidity and mortality [45]. Recently, a large study was performed in The Netherlands investigating microorganisms causing BSI in patients with and without cancer [46]. Again, Gram-positive isolates were more prevalent than Gram-negative isolates. Amongst Gram-positive organisms, the pathogens making up the top 3 were similar as found in the US study [43]. In cancer patients, twice as many BSIs were caused by Enterococcus faecalis and E. faecium. Amongst Gramnegative organisms, E. coli was the most common isolate in non-cancer patients. However, in cancer patients, twice as many BSIs were caused by $P$. aeruginosa and Enterobacter cloacae. Yeasts were grown twice as much from cancer patients as compared to cultures from non-cancer patients.

Different pathogens were cultured from blood cultures in different patient groups as shown from prevalence data obtained from different departments of a Dutch teaching hospital (unpublished data A.J.M. Loonen, G. Janssen, A.R. Jansz, and A.J.C. van den Brule, PAMM regional laboratories and Catharina Hospital, Eindhoven, The Netherlands). In Figure 1, the five departments which order most blood cultures are displayed, together with the top 5 of most frequently recovered pathogens from positive blood cultures. This again demonstrates 
that BSI can be caused by a broad scala of pathogens and the causative pathogens differ in distinct patient populations. A diagnostic test should be able to detect the most frequently encountered pathogens.

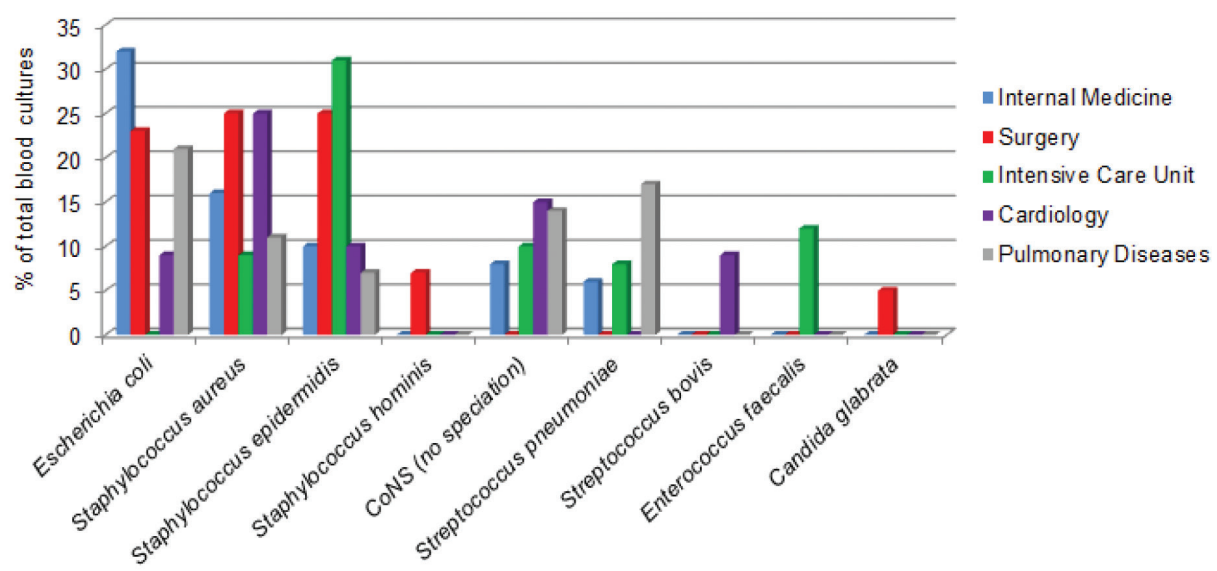

Figure 1. Overview of pathogens recovered from blood cultures of five departments of the Catharina Hospital (Eindhoven, The Netherlands).

The most frequently recovered pathogens (top 5) per department are depicted. Only S. aureus and $S$. epidermidis are detected from cultures collected from every department. (Unpublished data A.J.M. Loonen, G. Janssen, A.R. Jansz, and A.J.C. van den Brule, 2008).

$\mathrm{BSI}$ refers to the condition in which pathogens have entered the bloodstream. Bacteremia is a BSI caused by bacteria. Bacteremia in adults is characterized by low numbers of circulating bacteria, often 1-100 colony forming unit (CFU) /millilitre ( $\mathrm{ml})[47,48]$. However, other studies indicate a number of circulating bacteria ( $E$. coli and $S$. aureus) of less than $0.04 \mathrm{CFU} /$ $\mathrm{ml}$ [49]. BSI in young children are presumed to be characterized by higher bacterial loads (> $100 \mathrm{CFU} \mathrm{ml}$ ) as compared to bacteremia in adults $[48,50]$. Therefore, the volume of blood cultured is a critical factor in the detection of BSI [51-55]. The higher the blood volume cultured, the higher the detection rate of BSI.

It has been shown that inappropriate antibiotic therapy can result in higher mortality rates [3, 56], increased antibiotic resistance [57], and increased length of hospital stay [58]. Increased nosocomial BSI rates due to antibiotic resistant bacteria increase the total burden of disease $[59,60]$. Therefore, a fast diagnosis and adequate management of $\mathrm{BSI}$ is necessary for successful patient outcome. 


\section{Diagnostics of bloodstream infection}

\subsection{Blood cultures: the reference method}

Blood cultures are regarded as the "gold standard" for the detection of viable bacterial and fungal organisms from blood. Blood is usually collected from a single venipuncture and at least 2 culture bottles (aerobe and anaerobe) are filled with approximately 8-10 ml blood. However, this volume cannot be drawn from infants and children. The volume for pediatric blood cultures should be based on body weight and the estimated total blood volume in each child. Recent recommendations suggest the collection of no more than $1 \%$ of the total blood volume for children [2, 61].

When microorganisms start to grow, $\mathrm{CO}_{2}$ is produced, and the bottom of each culture bottle contains a $\mathrm{CO}_{2}$ indicator, which can change colour upon exposure. The automated, continuous-monitoring culture system detects this alteration. When a positive blood culture bottle is detected it is used for subsequent Gram staining, subculture on agar plates, biochemical testing, and antibiotic susceptibility testing (AST). The management of BSI is based on important parameters; 1) the detection and identification of (a) viable pathogen(s), and 2) the AST results. General principles and procedures for blood cultures from suspected sepsis patients are described in the Clinical and Laboratory Standards (CLSI) guidelines [2]. Despite the fact that improved media and automated culture systems, e.g., BacT/ALERT 3D (bioMérieux) and BACTEC (BD), have been developed and microorganisms are detected faster and more frequently [62], several factors are known which limit the clinical value of blood cultures.

The improved media in the culture bottles have indeed increased the diagnostic yields and decreased the time to detection $[63,64]$. However, not every culture bottle is immediately inserted in the automated culture system, as not every medical microbiology laboratory is in progress for 24 hours / 7 days a week. Several studies have demonstrated that a $24 \mathrm{~h}$ storage of blood culture bottles at room temperature negatively influenced the time to positivity [6567]. Furthermore, fast-growing strains (e.g. E. coli) and high bacterial loads inhibited bacterial detection due to exhausted $\mathrm{CO}_{2}$ production on which most culture systems are based. This indicates that delays from sample collection to insertion in the automated culture device affect the time to the detection of viable pathogens and might even lead to false-negative results.

Coagulase-negative staphylococci (CoNS) are normal habitants of human skin and mucous membranes. For decades they have been considered as culture contaminants (false-positive blood cultures). Since recent years, an important role of CoNS as pathogens has been recognized [68]. Several studies have provided guidelines on how to differentiate between contaminants and true pathogens [6,69]. Usually, when only one of four taken blood culture bottles is positive for CoNS, this blood culture is considered as contaminant. The 
microbiologist will also consider patient history to differentiate a true pathogen from possible contaminants. In case of CoNS it is important to know if a patient has indwelling medical devices, such as intravascular catheters, artificial heart valves, and orthopedic appliances [68]. Guidelines exist which describe approaches to limit blood culture contamination [2]. Thorough skin antisepsis, an adequate venipuncture method, and well-trained phlebotomist teams should reduce the chance of blood culture contamination [70-72].

Blood cultures are known to have a low sensitivity for slow-growing (e.g. Mycobacterium tuberculosis and most fungi) and fastidious microorganisms. Some examples of a highly fastidious microorganisms are Coxiella burnetii and Bartonella spp. which have been found in blood culture negative infective endocarditis [73, 74]. Furthermore, the sensitivity of blood cultures decreases significantly when antibiotic therapy has been started before blood samples are taken [75-77].

After the detection of microbial growth in a culture bottle, the pathogen(s) need(s) to be identified and the antibiotic susceptibility profile of the causative agent is needed. Subculturing from a positive blood culture takes at least 24 hours. For pathogen identification a wide variety of (semi-automated) biochemical tests are available. However, phenotypical tests (i.e. API tests) are slow and can display low specificity [78]. Clearly, Gram staining and fast biochemical tests (i.e. catalase test) can provide an initial diagnosis about the etiologic agent. However, detailed pathogen identification and AST take up several days and cause a delay in time-to-results.

\subsection{Identification of pathogens from positive blood cultures}

Recently, new techniques have become available for the rapid identification of pathogens from positive blood cultures. Most techniques are only suitable for identification of the causative pathogen and do not allow determination of antibiotic resistance. The antimicrobial susceptibility status of most locally prevalent bacterial and fungal species is largely predictable from past experience, and this information guides physicians to start empirical antibiotic treatment for patients with sepsis caused by these pathogens. Thus, rapid identification of pathogens commonly causing BSI is important and could allow species specific therapy to be started earlier.

The next paragraphs will highlight the latest technologies employed to aid in the identification of BSI pathogens. These techniques are based on the detection of proteins (matrix-assisted laser desorption ionization time-of-flight mass spectrometry (MALDI-TOF MS)), or DNA (fluorescence in situ hybridization (FISH) and (real-time) PCR). By using these techniques, results can be available within a few hours, from growth positive cultures, reducing turnaround time. Furthermore, fast identification of the disease causing pathogen allows more rapid initiation of adequate antibiotic therapy. 


\subsubsection{Protein based diagnostics}

\section{MALDI-TOF MS}

Matrix-assisted laser desorption ionization time-of-flight mass spectrometry (MALDI-TOF MS) is increasingly used for high-throughput identification of bacteria and yeast from agar plates [79]. Each individual microorganism contains an unique protein profile (fingerprint) that allows identification of microorganisms to the species level. Figure 2 illustrates the principle of MALDI-TOF MS. In 1975, Anhalt et al. were the first to describe the use of mass spectrometry for bacterial identification [80]. Several studies have been published that show that MALDI-TOF MS can be employed for identification of a wide variety of pathogens, including Gram-positive and Gram-negative bacteria [81-84], non-fermenting bacteria [85], anaerobes [86, 87], and yeasts [88].

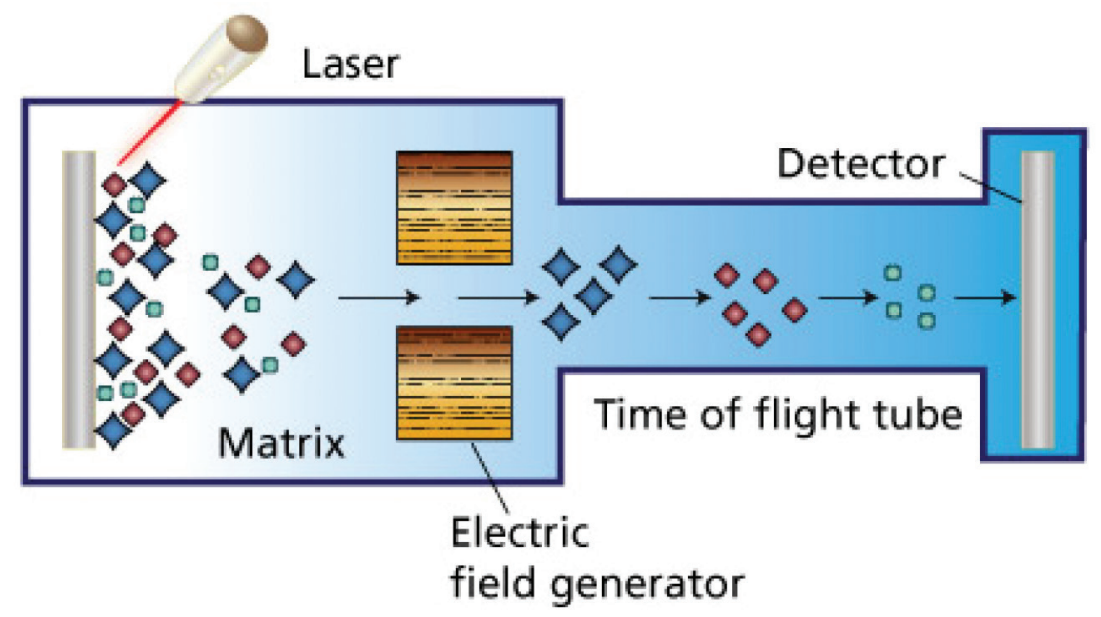

Figure 2. Overview of the general principle of MALDI-TOF MS.

A colony is picked from an agar plate, spotted on a steel target plate, and overlayed with matrix. The laser hits the samples and charged ions of various sizes are generated on the sample slide. Ions with a smaller mass to charge value (lighter ions) move faster through the flight tube until they reach the detector. A peak pattern is generated and this pattern is in most cases specific per bacterial species. This figure was redrawn from sigmaaldrich.com.

The main advantage of the use of MALDI-TOF is that results can be available within minutes and in most cases no sample preparation is needed. For fungal identification sample pretreatment with formic acid might be necessary. One limitation of the method is that for a successful protein profile at least $>10^{7} \mathrm{CFU} / \mathrm{ml}$ need to be present in the sample. Christner et al. have shown that when only $10^{6} \mathrm{CFU} / \mathrm{ml}$ are present in a sample this peak pattern resembles that of a negative culture [89]. This indicates that a pre-culture step remains crucial 
for optimal results. In addition, MALDI-TOF MS has been shown to encounter difficulties when identifying viridans streptococci [79, 90, 91] and mixed infections [90, 91].

Aside from bacterial identification, the first reports are available in which successful identification of antibiotic resistance, e.g. methicillin resistance in $S$. aureus, is described $[92$, 93]. Schaumann et al. described a first effort towards the discrimination of betalactamase-producing clinical isolates of Enterobacteriaceae and $P$. aeruginosa by MALDITOF MS [94]. The role of MALDI-TOF MS in the determination of antibiotic resistance is not yet reliable for routine microbiological diagnostics. It is expected that implementation of this technique, in diagnostics of BSI, will result in an increased number of patients on adequate antimicrobial treatment within 24 hours of onset of disease [95].

\subsubsection{DNA based diagnostics}

\section{Fluorescence in situ hybridization}

Fluorescence in situ hybridization (FISH) is a technique in which fluorochrome-labelled oligonucleotide probes are used to target rRNA molecules of different microorganisms. Subsequently, these probes are visualised by microscopy (Figure 3). FISH can be used on colonies as well as positive blood cultures.

FISH assays were developed for the detection of $S$. aureus and CoNS [96, 97], Enterococcus spp. [98, 99], Streptococcus spp. [98], P. aeruginosa [100], E. coli [100], Brucella spp. [101], and fungi [102]. Peters et al. developed a FISH protocol which allows identification of bacteria and yeast from positive blood cultures within 1 hour [103]. Although this technique seems promising, it is hampered by the permeabilization of the cell wall during preparation, hybridisation conditions, and the available probe collection. Due to the limited probe collection, species-specific identification for all BSI causing pathogens is not feasible. 


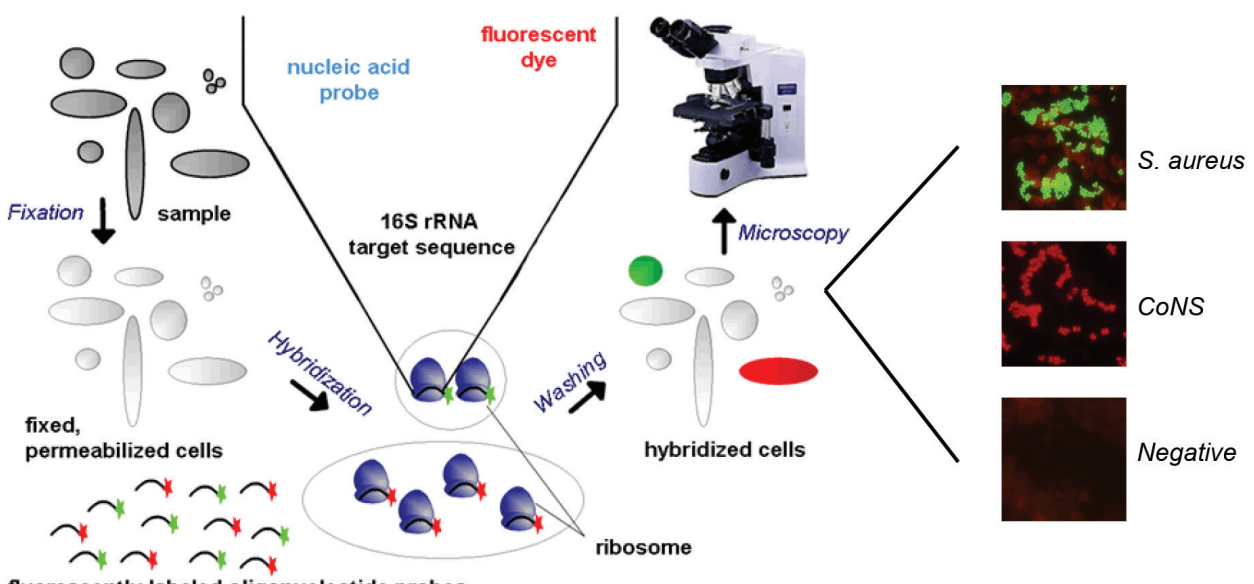

fluorescently labeled oligonucleotide probes

Figure 3. Overview of the FISH workflow.

When a positive blood culture bottle is recovered from the blood culture system a Gram strain is performed. Based on the Gram stain a suitable FISH probe-set can be chosen. First, the sample is fixed on a glass slide and permeabilized. Next, fluorescent-labelled probes are added (hybridization) and these can be visualised by using a microscope. This figure was redrawn from wrhsrc.oregonstate. edu.

\section{PCR based technologies}

\section{Conventional PCR}

The polymerase chain reaction (PCR) is one of the most important nucleic acid amplification techniques, in the laboratory, available today and can be used for pathogen identification from both colonies as well as blood cultures. It was already developed 25 years ago, by Mullis and Faloona [104]. PCR is an enzyme-driven process, and makes it possible to turn a few DNA or RNA molecules into large quantities. First, DNA is denatured, and in the presence of nucleotides, buffer and primers, DNA can be copied by using a heat-stable polymerase (Figure 4). Primers are short DNA oligomers that are complementary to the ends of a target sequence. 

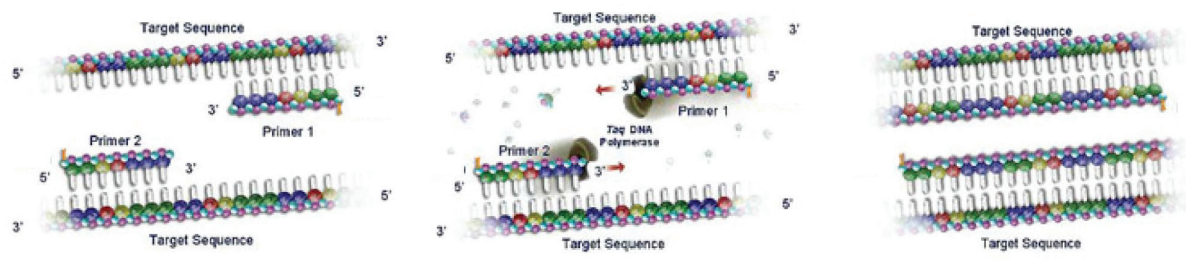

Figure 4. General principle of the polymerase chain reaction (PCR).

DNA is heated to $95^{\circ} \mathrm{C}$ to denature the DNA strands. Subsequently, the temperature is lowered (usually to $60^{\circ} \mathrm{C}$, this depends on the primer sequences) to allow the primers to bind to the complementary sequences in the target (left). At $72^{\circ} \mathrm{C}$, Taq polymerase extends the complementary strands from the primers (middle) by using nucleotides. The DNA target has been doubled (right), and the second cycle can start. This results in an exponential increase of target DNA molecules. This figure was redrawn from molecular.roche.com.

In the conventional PCR procedure amplification and detection are separated. To check whether the PCR generated the anticipated DNA fragment, agarose gelelectrophoresis is employed for size separation of the PCR fragments. Before size separation, DNA has to be stained (ethidiumbromide or Midori Green). When exposed to ultraviolet light, these dyes will fluoresce and DNA will be visible [105].

\section{Microarray}

Recently, a new platform was launched, Prove-It Sepsis (Mobidiag, Helsinki, Finland). The assay constitutes of a broad-range PCR with a microarray-based platform that is designed to identify 60 bacterial pathogens, 13 fungal pathogens, and mecA (methicillin resistance) from positive blood cultures [106]. After growth positivity, results can be available within 3.5 hours. Tissari et al. evaluated the clinical performance of Prove-It Sepsis and found a sensitivity and specificity of $94.7 \%$ and $98.8 \%$, respectively [106].

\section{Sequencing}

DNA sequencing (Sanger) is a technology that can be employed to determine unknown amplified DNA fragments [107]. The Sanger sequencing method is well known and the procedure is similar to conventional PCR. The main difference is that, in addition to the other compounds, modified nucleotides (dideoxy-nucleotides) are used which terminate DNA strand elongation. The modified nucleotides are fluorescently labelled for detection in automated sequencing machines [108]. Currently, newer sequencing technologies are emerging. The use of next generation sequencing for BSI diagnostics needs to be demonstrated in future studies. 


\section{Real-time PCR}

In real-time PCR, nucleic acid amplification and detection are performed simultaneously within the same vial. This approach reduces contamination risks significantly. This technique is easy to perform, fast, and characterized by high sensitivity and specificity making it a suitable alternative to use in the clinical microbiology setting [109]. In the real-time PCR approach, conventional PCR can be combined with fluorescent probe detection of the amplified target DNA. The probe is an oligonucleotide with a fluorescent label (i.e. FAM or VIC) that consists of a reporter dye that is quenched in proximity of a quencher (i.e. BHQ or TAMRA). As Taq polymerase extends the new DNA strand, it cleaves the probe releasing the reporter from the quencher. The resulting fluorescence is measured in each PCR cycle and plotted against the cycle number [110]. Another way to visualize DNA in a real-time setting is by using SYBR Green. SYBR Green binds all double-stranded DNA, making it unspecific, and detection is monitored by measuring the increase in fluorescence throughout the PCR cycles. By using melting curve analysis, it is possible to differentiate between targets.

After its first development, PCR was considered a promising approach for diagnosis of infectious diseases [111, 112]. PCR turned out to be a major development in the area of molecular diagnostics. Various studies have been published describing real-time PCR based assays for the identification of pathogens from clinical samples. These are based either on broad-range (e.g. 16S or 18S rDNA), pathogen-specific or multiplex detection [113-116]. When using universal amplification, DNA sequencing analysis can provide identification within 4 hours after the initial positive PCR result. This approach is useful when unexpected pathogens are recovered by conventional methods or confirmation of results is necessary. In previous studies, both conventional as well as real-time PCR assays were developed for the detection of CoNS, S. aureus, and/or methicillin resistant S. aureus (MRSA) [117-122], S. pneumoniae [123, 124], E. faecalis [125], Neisseria meningitides [124], Aspergillus spp. [126], and Candida spp. [127]. The use of pathogen specific assays proves challenging as the wide variety of causative pathogens would lead to a complex combination of assays [128]. Pathogen-specific assays might be clinically valuable when combined with the clinical presentation of a patient or Gram staining, as it is then known which microorganism is most likely present in the sample. Broad-range or multiplex assays enable detection of a broad scala of pathogens, this makes these assays more suitable for BSI diagnostics [129, 130].

Overall, the described technologies allow pathogen identification results to be available within several hours after growth positive blood cultures are detected by the automated culture system. When sequencing is necessary, time to results increase significantly. More rapid pathogen detection and identification can be achieved when whole blood is used, because the time consuming pre-culture steps can then be eliminated. 


\subsection{PCR based detection and identification of pathogens directly from whole blood}

Direct detection from blood, and thus eliminating the time consuming culturing step, will result in faster pathogen detection and identification. Molecular assays can overcome the main limitations associated with the conventional blood culture based approach such as decreased sensitivity for fastidious or slow-growing pathogens, and prior antibiotic treated patients. Pathogen detection and identification can be available within a few hours, but first the crucial step of sample preparation will be discussed in this non-comprehensive overview.

\subsubsection{Sample preparation}

A crucial step in the whole process of pathogen detection using DNA based assays is sample preparation [131]. Cultured specimens contain high bacterial or fungal loads, but compounds present in the culture media can interfere in the downstream PCR reaction (e.g. charcoal, sodium polyanethol sulfonate (SPS, anticoagulant)) [132]. Due to the presence of high pathogen loads, the nucleic acid extraction procedure is less important. By applying a few washing steps (water), subsequent dilution, and heating of the sample, PCR results can already be obtained. When PCR is performed on positive culture material a reduction in timeto-result can be achieved [133].

The first attempts to directly detect bacteria in whole blood date from 1993 [134, 135]. The numbers of clinical samples were small, but the sensitivities were $80 \%$ and $92 \%$ as compared to blood culture. As Gram-positive bacteria contain another cell-wall composition as compared to Gram-negative bacteria or fungi it is difficult to apply one nucleic acid isolation procedure for all these different pathogens [136].

Several challenges need to be dealt with when pathogens need to be detected directly from whole blood of suspected BSI patients. First, the low pathogen load in whole blood results in low amounts of pathogen DNA versus human DNA [137]. Second, whole blood contains inhibitory compounds which can interfere in the PCR (i.e. hemoglobin) [138]. Third, commercial PCR and DNA isolation reagents can be contaminated with bacterial of fungal DNA, leading to false-positive results [139, 140].

A frequently used nucleic acid isolation procedure in the molecular diagnostic laboratory is the NucliSENS EasyMAG (Biomérieux, Marcy l'Etoile, France). It is an automated system for total nucleic acid extraction from a variety of sample types and volumes. Whole blood can be used $(200 \mu \mathrm{l})$ which is first lysed. Then, magnetic silica particles (beads) are added to which total nucleic acids can bind. Denatured proteins and other compounds are washed away and finally bound DNA and RNA are removed from the beads by using a buffer with a low-salt concentration.

To circumvent the difficulties encountered when working with whole blood (high human DNA levels), pathogen enrichment might be a solution. Recently, commercial pathogen DNA 
isolation methods became available; SeptiFAST (Roche Diagnostics, Mannheim, Germany), Looxter (SIRS-Lab, Jena, Germany) and MolYsis (Molzym GmbH, Bremen, Germany). Looxter allows total genomic DNA isolation from $5 \mathrm{ml}$ whole blood, followed by the specific binding of prokaryotic DNA to a column. Specific bacterial DNA isolation is based upon the presence of non-methylated CpG dinucleotides in the bacterial but not in the human DNA [141, 142]. Most eukaryotic DNA is removed by washing the column. Thus, Looxter concentrates the prokaryotic DNA and eliminates a high amount of background DNA. The MolYsis method can process 1 to $5 \mathrm{ml}$ whole blood. This method enables selective lysis of human cells, followed by the enzymatic degradation of human DNA and DNA of compromised pathogens. Subsequently, bacteria are enriched by centrifugation and lysed. Finally, pathogen DNA is isolated. SeptiFAST does not allow specific isolation of only pathogen DNA. It isolates total nucleic acids from the whole blood sample and might therefore be comparable with the EasyMAG. The only difference is that the EasyMAG can process only $200 \mu$, while SeptiFAST enables processing of $1.5 \mathrm{ml}$ whole blood. It has been shown by Handschur et al. that human DNA can interfere in a $16 \mathrm{~S}$ rDNA real-time PCR, and when human DNA is removed from the sample these unspecific signal are eliminated [137]. Other studies have shown that when combining a human DNA removal protocol (MolYsis or Looxter) with EasyMAG sensitive results can be obtained as well $[143,144]$. A detection limit of $10-100 \mathrm{CFU} / \mathrm{ml}$ in whole blood was obtained. This pathogen enrichment strategy can be applied to other clinical specimens as well (i.e. biopsy, pleural fluid, and cerebrospinal fluid) [136]. MolYsis and Looxter allow removal of at least $90 \%$ of the human DNA [141]. The perfect method which works optimal for all pathogens and every clinical specimen still needs to be developed.

Besides the commercially available DNA isolation methods, new pathogen enrichment strategies are being established. Smith et al, have developed a solution-based hybridisation method for the enrichment of pathogen DNA from samples that contain human DNA [145]. This technique utilises off-the-shelf reagents and home-made probes which can be made using whole-genome DNA from any organism of interest. This is an example of an approach which might result in optimal enrichment of every pathogen. There seems to be a continuous development of techniques which further optimise sample preparation.

\subsubsection{Multiplex PCR assays for detecting pathogen DNA}

To date, several commercially available assays have been developed, which allow detection of the most frequently encountered and clinically relevant pathogens causing BSI (Table 2). SeptiFAST (Roche Diagnostics, Mannheim, Germany) ensures detection of up to 25 pathogens and mecA (methicillin resistance) from whole blood by using real-time PCR coupled to melting curve analysis. Results can be available in less than 6 hours. Most studies have demonstrated the benefits of using SeptiFAST in addition to blood culture $[129,146$, 147], especially in antibiotic pre-treated patients. 
SepsiTest (Molzym GmbH, Bremen, Germany) is a real-time PCR test in which 16S rDNA and 18S rDNA are being amplified by using broad-range primers and SYBR Green. Subsequent sequencing analysis needs to be performed to identify the microorganism in the sample. By using SepsiTest, more than 345 bacteria and fungi can be detected from whole blood samples within 8 hours. In a study performed by Kuhn et al., SepsiTest was used to diagnose infectious endocarditis and proved to be twice as sensitive as compared to culture methods [148]. Wellinghausen et al. have shown that the concordance of SepsiTest with blood culture is $86 \%$ [149].

The VYOO method (SIRS-Lab GmbH, Jena, Germany) simultaneously detects 34 bacterial and 6 fungal species as well as 5 of the most frequent resistance markers from whole blood within 8 hours. First, a multiplex PCR is performed followed by microarray analysis. Fitting et al. found a concordance in bacterial identification between microbiology and the VYOO test of $46.2 \%$ [150]. Based on the results obtained in their first patient cohort, they decided not to continue the study.

Seegene's MagicPlex Sepsis Test (Seoul, Korea), allows detection of up to 90 pathogens and 3 resistance markers (methicillin and vancomycin A and B) from whole blood. First an amplicon bank needs to be created by conventional PCR and subsequent signal amplification serves as primary detection system. Next, 27 pathogens can be identified to the species level: 21 bacterial species and 6 fungal species. The results can be available within 6 hours, including pathogen DNA isolation. Serra et al. clinically evaluated the MagicPlex Sepsis Test to detect candida DNA in pediatric patients [151]. They considered the test as an effective diagnostic tool for diagnosing invasive candidiasis along with conventional cultures. No studies have been published in which the MagicPlex Sepsis Test has been evaluated in adult BSI patients.

Table 2. Overview of pathogen detection and identification tests for whole blood.

\begin{tabular}{lllll}
\hline Test (company) & $\begin{array}{l}\text { Pathogen DNA } \\
\text { enrichment }\end{array}$ & $\begin{array}{l}\text { \# of pathogens } \\
\text { detected }\end{array}$ & $\begin{array}{l}\text { \# of resistance } \\
\text { markers }\end{array}$ & Time to results \\
$\begin{array}{l}\text { SeptiFAST } \\
\text { (Roche Diagnostics) }\end{array}$ & No & 25 & 1 (mecA) & 6 hours \\
$\begin{array}{l}\text { SepsiTest (Molzym) } \\
\text { VYOO (SIRS-Lab) }\end{array}$ & Yes, MolYsis & 345 & 0 & 8 hours \\
& Yes, Looxter & 40 & 5 (mecA, vanA, 8 hours \\
vanB, blaSHV, & \\
MagicPlex Sepsis & Yes, MolYsis & $90(27$ to species & $\begin{array}{l}3 \text { (mecA, vanA, } 6 \text { hours } \\
\text { vanB) }\end{array}$ & \\
\hline
\end{tabular}

MecA: methicillin resistance, vanA and vanB: vancomycin resistance, blaSHV and blaCTX-M: $\beta$-lactamases. 
The clinical interpretation of a positive PCR signal with a negative culture result is still unclear. The PCR results might be correct and be the result of the higher sensitivity of the PCR for slow-growing microorganisms, or pathogens that do not grow due to presence of antibiotics. However, the PCR signal might be false-positive and due to detection of dead microorganisms or contamination. Due to the lack of an appropriate "gold standard" to compare to, the (discordant) results of new diagnostics methods need to be related to the clinical context of the patient. In such studies, patient outcome and possible modification in treatment regime of a patient, based on results from new diagnostic tests, need to be monitored.

\subsubsection{Detection of active BSI}

As bacteremia is defined as the presence of viable bacteria in the bloodstream, it might be important to assess the viability of the bacteria in blood samples. Classical methods for the detection of $\mathrm{BSI}$ are based on culturing which intrinsically confirm the viability of the microorganisms. However, culturing methods could be hampered by the fact that many pathogens can, when exposed to stress (i.e. starvation), enter either a dormancy state or die [152]. In this viable but non-culturable state, the pathogens are not able to form colonies on solid media but can become infective again when exposed to a good environment.

The presence of pathogen DNA in a clinical sample does not immediately provide information about the viability status of that pathogen as it can originate from both living and dead microorganisms [153-156]. The clinical significance of pathogen DNA detection is therefore not clear.

It has been shown, by others, that DNA detection in time (measured for several days), after initiation of antibiotic therapy, can be used to provide information about the rate of bacterial clearance $[157,158]$. The cycle threshold (Ct) values in the real-time PCR increase, indicating a lower amount of pathogen DNA present in the sample. Furthermore, it has been shown by Peters et al. that DNA load (related to the amount of pathogens) in blood can be used as a marker of severity of infection in patients with community acquired pneumonia [159]. This indicates that the amount of pathogen DNA present in a patient needs to be monitored in time to provide clinical useful information (e.g. initiation of appropriate therapy). Currently, most (commercially) available molecular sepsis tests are only used to detect (absence or presence) and identify pathogens from whole blood at a single moment in time. There are ways to ensure detection of DNA solely from living microorganisms. The dye propidium monoazide (PMA) is membrane-impermeable and can only selectively penetrate pathogens with compromised membranes, which can be considered dead. Subsequently, PMA binds to the DNA thereby inhibiting PCR amplification [160].

Other molecules, such as messenger RNA (mRNA) or ribosomal RNA (rRNA), might also be more suitable to determine pathogen viability status as compared to pathogen DNA detection 
$[161,162]$. It has been shown that DNA can be detected after viable organisms have been eradicated [163]. It is clear that the clinical value of pathogen DNA detection from whole blood needs further investigation.

\section{Outline of the thesis}

It is highly unlikely that culture techniques can still be improved to significantly decrease timeto- results. Therefore, the research presented in this thesis focussed on improving turnaround times by using new technologies for detection and identification of microorganisms (directly) from clinical specimens of BSI patients. The techniques used for this study, needed to be fast, robust, sensitive and specific.

A first step forward, to decrease time-to-result, can be achieved by using faster identification technologies on growth positive blood cultures. Moreover, eliminating culture steps will lead to the most optimal turnaround time. This means that using molecular assays directly on whole blood might be promising. When using molecular diagnostics, sample preparation is crucial and needs careful investigation, as it is known that no single pathogen nucleic acid isolation procedure is optimal for the different causative pathogens of BSI. Furthermore, large whole blood volumes need to be used to have optimal detection rates as compared to blood cultures. Whole blood contains lots of human cells, and as a consequence human DNA, and often only few microorganisms. Pathogen enrichment technologies for whole blood might be the solution for this problem.

Various biomarkers might have predictive value for BSI in patients with (suspected) sepsis. Nonetheless, the single biomarker which can predict BSI in different patient populations remains to be found. Furthermore, it would be interesting to use biomarkers as patient preselection tools for the new and still expensive molecular assays. When BSI can be predicted, molecular assays can provide results within hours as compared to culture methods, which take several days. Although molecular assays are promising, their performance needs thorough investigation. The main objective of this research was to improve laboratory diagnostics of BSI.

In the first part of this thesis the main focus was to decrease time-to-results by using faster pathogen identification technologies on (positive) blood cultures. First, sensitive and specific real-time PCR assays, based on the tuf gene, were developed enabling accurate and fast detection of pathogens from different clinical specimens indicative for Gram-positive cocci, as these microorganisms are most frequently encountered in de medical microbiology laboratory (Chapter 2).

Second, we evaluated a number of bacterial DNA extraction methods for blood culture material to obtain optimal results. Then, we attempted to accelerate the direct identification of $S$. aureus and CoNS from blood culture bottles by reducing culture times, isolate bacterial DNA with the best method, and using real-time PCR analysis (Chapter 3). 
As the clinical relevance of CoNS infections is not clear, we performed a comparative study using phenotypic, genotypic and proteomics methods to find the best identification method for CoNS spp. available today (Chapter 4). Next, the best method found, MALDI-TOF MS, was used to identify more than 300 CoNS strains obtained from bacteremia patients of 2 academic centres (Rotterdam and Maastricht). The bacterial identification was linked to clinical parameters to investigate the clinical relevancy of this bacterial identification (Chapter 5).

After that, we evaluated processing methods and the effect of reduced culture times for faster direct identification of pathogens from blood cultures by using MALDI-TOF MS (Chapter 6). In this way, pathogens are identified faster and this might improve patient care.

In the second part of the thesis, we focussed on direct pathogen detection and identification from whole blood. Polaris, a novel non-enzymatic method for enrichment of pathogen DNA from large volumes of whole blood was developed and evaluated in comparison with available methods (i.e. MolYsis and EasyMAG). This was done for commonly detected microorganisms from blood of sepsis patients (Chapter 7).

In Chapter 8, several biomarkers were investigated for their ability to predict BSI in patients showing two or more SIRS symptoms. These patients originated from the emergency department. Subsequently, molecular assays were investigated in comparison to blood culture to determine the additional value of these assays.

RNA was investigated as a viability marker, as compared to DNA, for $S$. aureus after antibiotic treatment in both culture broth as well as whole blood (Chapter 9).

In Chapter 10, a summary is provided about the preceding findings and future perspectives are given. 


\section{References}

1. Libman, E., Weitere Mitteilungen über die Streptokokken-enteritis bei Säuglingen. Zentralbl Bakteriology, 1897. XXII: p. 376.

2. Institute, C.a.L.S., Principles and Procedures for Blood Cultures; Approved Guidline, in CLSI Document M47-A (ISBN 1-56238-641-7). 2007.

3. Kumar, A., et al., Initiation of inappropriate antimicrobial therapy results in a fivefold reduction of survival in human septic shock. Chest, 2009. 136(5): p. 1237-48.

4. Lim, S.M. and S.A. Webb, Nosocomial bacterial infections in Intensive Care Units. I: Organisms and mechanisms of antibiotic resistance. Anaesthesia, 2005. 60(9): p. 887-902.

5. Spraycar, M., in Stedman's Medical Dictionary. 1995, Williams \& Wilkins.

6. Weinstein, M.P., et al., The clinical significance of positive blood cultures in the 1990s: a prospective comprehensive evaluation of the microbiology, epidemiology, and outcome of bacteremia and fungemia in adults. Clin Infect Dis, 1997. 24(4): p. 584-602.

7. Bone, R.C., et al., Definitions for sepsis and organ failure and guidelines for the use of innovative therapies in sepsis. The ACCP/SCCM Consensus Conference Committee. American College of Chest Physicians/Society of Critical Care Medicine. Chest, 1992. 101(6): p. 1644-55.

8. Levy, M.M., et al., 2001 SCCM/ESICM/ACCP/ATS/SIS International Sepsis Definitions Conference. Crit Care Med, 2003. 31(4): p. 1250-6.

9. Lever, A. and I. Mackenzie, Sepsis: definition, epidemiology, and diagnosis. BMJ, 2007. 335(7625): p. 879-83.

10. Valles, J., et al., Evolution over a 15-year period of clinical characteristics and outcomes of critically ill patients with community-acquired bacteremia. Crit Care Med, 2013. 41(1): p. 76-83.

11. van Gestel, A., et al., Prevalence and incidence of severe sepsis in Dutch intensive care units. Crit Care, 2004. 8(4): p. R153-62.

12. CDC, Report of mortality statistics. Monthly Vital Statistics Report, 1992. 40(11): p. 1-23.

13. Alberti, C., et al., Epidemiology of sepsis and infection in ICU patients from an international multicentre cohort study. Intensive Care Med, 2002. 28(2): p. 108-21.

14. Angus, D.C. and R.S. Wax, Epidemiology of sepsis: an update. Crit Care Med, 2001. 29(7 Suppl): p. S109-16.

15. Garrouste-Orgeas, M., et al., Excess risk of death from intensive care unit-acquired nosocomial bloodstream infections: a reappraisal. Clin Infect Dis, 2006. 42(8): p. 1118-26.

16. Kim, P.W., et al., Risk of mortality with a bloodstream infection is higher in the less severely ill at admission. Am J Respir Crit Care Med, 2005. 171(6): p. 616-20.

17. Engel, C., et al., Epidemiology of sepsis in Germany: results from a national prospective multicenter study. Intensive Care Med, 2007. 33(4): p. 606-18.

18. Dalton, W.S. and S.H. Friend, Cancer biomarkers--an invitation to the table. Science, 2006. 312(5777): p. 1165-8.

19. Petrikkos, G.L., et al., Value of measuring serum procalcitonin, C-reactive protein, and mannan antigens to distinguish fungal from bacterial infections. Eur J Clin Microbiol Infect Dis, 2005. 24(4): p. 272-5.

20. Standage, S.W. and H.R. Wong, Biomarkers for pediatric sepsis and septic shock. Expert Rev Anti Infect Ther, 2011. 9(1): p. 71-9.

21. Uusitalo-Seppala, R., et al., Early detection of severe sepsis in the emergency room: diagnostic value of plasma C-reactive protein, procalcitonin, and interleukin-6. Scand J Infect Dis, 2011. 43(11-12): p. 883-90.

22. Mencacci, A., et al., Procalcitonin predicts real-time PCR results in blood samples from patients with suspected sepsis. PLoS One, 2012. 7(12): p. e53279.

23. de Jager, C.P., et al., Lymphocytopenia and neutrophil-lymphocyte count ratio predict bacteremia better than conventional infection markers in an emergency care unit. Crit Care, 2010. 14(5): p. R192.

24. Backes, Y., et al., Usefulness of suPAR as a biological marker in patients with systemic inflammation or infection: a systematic review. Intensive Care Med, 2012. 38(9): p. 1418-28. 
25. Donadello, K., et al., suPAR as a prognostic biomarker in sepsis. BMC Med, 2012. 10: p. 2.

26. Haupt, T.H., et al., Plasma suPAR levels are associated with mortality, admission time, and Charlson Comorbidity Index in the acutely admitted medical patient: a prospective observational study. Crit Care, 2012. 16(4): p. R130.

27. Hoenigl, M., et al., Diagnostic accuracy of soluble urokinase plasminogen activator receptor (sUPAR) for prediction of bacteremia in patients with systemic inflammatory response syndrome. Clin Biochem, 2013. 46(3): p. 225-9.

28. Koch, A., et al., Circulating soluble urokinase plasminogen activator receptor is stably elevated during the first week of treatment in the intensive care unit and predicts mortality in critically ill patients. Crit Care, 2011. 15(1): p. R63.

29. Meisner, M., et al., Comparison of procalcitonin (PCT) and C-reactive protein (CRP) plasma concentrations at different SOFA scores during the course of sepsis and MODS. Crit Care, 1999. 3(1): p. 45-50.

30. Silvestre, J., L. Coelho, and P. Povoa, Should C-reactive protein concentration at ICU discharge be used as a prognostic marker? BMC Anesthesiol, 2010. 10: p. 17.

31. Gogos, C.A., et al., Pro-versus anti-inflammatory cytokine profile in patients with severe sepsis: a marker for prognosis and future therapeutic options. J Infect Dis, 2000. 181(1): p. 176-80.

32. Simon, L., et al., Serum procalcitonin and C-reactive protein levels as markers of bacterial infection: a systematic review and meta-analysis. Clin Infect Dis, 2004. 39(2): p. 206-17.

33. Tang, B.M., et al., Accuracy of procalcitonin for sepsis diagnosis in critically ill patients: systematic review and meta-analysis. Lancet Infect Dis, 2007. 7(3): p. 210-7.

34. Uzzan, B., et al., Procalcitonin as a diagnostic test for sepsis in critically ill adults and after surgery or trauma: a systematic review and meta-analysis. Crit Care Med, 2006. 34(7): p. 1996-2003.

35. Reinhart, K., et al., New approaches to sepsis: molecular diagnostics and biomarkers. Clin Microbiol Rev, 2012. 25(4): p. 609-34.

36. Zahorec, R., Ratio of neutrophil to lymphocyte counts--rapid and simple parameter of systemic inflammation and stress in critically ill. Bratisl Lek Listy, 2001. 102(1): p. 5-14.

37. Roldan, A.L., et al., Cloning and expression of the receptor for human urokinase plasminogen activator, a central molecule in cell surface, plasmin dependent proteolysis. EMBO J, 1990. 9(2): p. 467-74.

38. Ploug, M., et al., Cellular receptor for urokinase plasminogen activator. Carboxyl-terminal processing and membrane anchoring by glycosyl-phosphatidylinositol. J Biol Chem, 1991. 266(3): p. 1926-33.

39. Eugen-Olsen, J., suPAR - a future risk marker in bacteremia. J Intern Med, 2011. 270(1): p. 2931.

40. Thuno, M., B. Macho, and J. Eugen-Olsen, suPAR: the molecular crystal ball. Dis Markers, 2009. 27(3): p. 157-72.

41. Mizukami, I.F., et al., Enzyme-linked immunoabsorbent assay detection of a soluble form of urokinase plasminogen activator receptor in vivo. Blood, 1995. 86(1): p. 203-11.

42. Koch, A. and F. Tacke, Risk stratification and triage in the emergency department: has this become 'suPAR' easy? J Intern Med, 2012. 272(3): p. 243-6.

43. Wisplinghoff, H., et al., Nosocomial bloodstream infections in US hospitals: analysis of 24,179 cases from a prospective nationwide surveillance study. Clin Infect Dis, 2004. 39(3): p. 309-17.

44. Annane, D., E. Bellissant, and J.M. Cavaillon, Septic shock. Lancet, 2005. 365(9453): p. 6378.

45. Shoham, S. and S. Marwaha, Invasive fungal infections in the ICU. J Intensive Care Med, 2010. 25(2): p. 78-92.

46. Bos, M.M., et al., Bloodstream infections in patients with or without cancer in a large community hospital. Infection, 2013.

47. Mermel, L.A. and D.G. Maki, Detection of bacteremia in adults: consequences of culturing an inadequate volume of blood. Ann Intern Med, 1993. 119(4): p. 270-2.

48. Yagupsky, P. and F.S. Nolte, Quantitative aspects of septicemia. Clin Microbiol Rev, 1990. 3(3): p. 269-79. 
49. Jonsson, B., A. Nyberg, and C. Henning, Theoretical aspects of detection of bacteraemia as a function of the volume of blood cultured. APMIS, 1993. 101(8): p. 595-601.

50. Kennaugh, J.K., et al., The effect of dilution during culture on detection of low concentrations of bacteria in blood. Pediatr Infect Dis, 1984. 3(4): p. 317-8.

51. Bouza, E., et al., Is the volume of blood cultured still a significant factor in the diagnosis of bloodstream infections? J Clin Microbiol, 2007. 45(9): p. 2765-9.

52. Cockerill, F.R., 3rd, et al., Optimal testing parameters for blood cultures. Clin Infect Dis, 2004. 38(12): p. 1724-30.

53. Gonsalves, W.I., et al., Effects of volume and site of blood draw on blood culture results. J Clin Microbiol, 2009. 47(11): p. 3482-5.

54. Hall, M.M., D.M. IIstrup, and J.A. Washington, 2nd, Effect of volume of blood cultured on detection of bacteremia. J Clin Microbiol, 1976. 3(6): p. 643-5.

55. Tenney, J.H., et al., Controlled evaluation of the volume of blood cultured in detection of bacteremia and fungemia. J Clin Microbiol, 1982. 15(4): p. 558-61.

56. Valles, J., et al., Community-acquired bloodstream infection in critically ill adult patients: impact of shock and inappropriate antibiotic therapy on survival. Chest, 2003. 123(5): p. 161524.

57. Harbarth, S., et al., Inappropriate initial antimicrobial therapy and its effect on survival in a clinical trial of immunomodulating therapy for severe sepsis. Am J Med, 2003. 115(7): p. 52935.

58. Shorr, A.F., et al., Inappropriate antibiotic therapy in Gram-negative sepsis increases hospital length of stay. Crit Care Med, 2011. 39(1): p. 46-51.

59. Ammerlaan, H.S., et al., Secular trends in nosocomial bloodstream infections: antibioticresistant bacteria increase the total burden of infection. Clin Infect Dis, 2013. 56(6): p. 798805 .

60. de Kraker, M.E., et al., The changing epidemiology of bacteraemias in Europe: trends from the European Antimicrobial Resistance Surveillance System. Clin Microbiol Infect, 2012.

61. Kellogg, J.A., J.P. Manzella, and D.A. Bankert, Frequency of low-level bacteremia in children from birth to fifteen years of age. J Clin Microbiol, 2000. 38(6): p. 2181-5.

62. Engler, H.D., G.A. Fahle, and V.J. Gill, Clinical evaluation of the BacT/Alert and isolator aerobic blood culture systems. Am J Clin Pathol, 1996. 105(6): p. 774-81.

63. McDonald, L.C., et al., Clinical importance of increased sensitivity of BacT/Alert FAN aerobic and anaerobic blood culture bottles. J Clin Microbiol, 1996. 34(9): p. 2180-4.

64. Weinstein, M.P., et al., Controlled evaluation of BacT/Alert standard aerobic and FAN aerobic blood culture bottles for detection of bacteremia and fungemia. J Clin Microbiol, 1995. 33(4): p. 978-81.

65. Akan, O.A. and E. Yildiz, Comparison of the effect of delayed entry into 2 different blood culture systems (BACTEC 9240 and BacT/ALERT 3D) on culture positivity. Diagn Microbiol Infect Dis, 2006. 54(3): p. 193-6.

66. Sautter, R.L., et al., Effects of delayed-entry conditions on the recovery and detection of microorganisms from BacT/ALERT and BACTEC blood culture bottles. J Clin Microbiol, 2006. 44(4): p. 1245-9.

67. Schwetz, I., et al., Delayed processing of blood samples influences time to positivity of blood cultures and results of Gram stain-acridine orange leukocyte Cytospin test. J Clin Microbiol, 2007. 45(8): p. 2691-4.

68. Piette, A. and G. Verschraegen, Role of coagulase-negative staphylococci in human disease. Vet Microbiol, 2009. 134(1-2): p. 45-54.

69. Bates, D.W. and T.H. Lee, Rapid classification of positive blood cultures. Prospective validation of a multivariate algorithm. JAMA, 1992. 267(14): p. 1962-6.

70. Hall, K.K. and J.A. Lyman, Updated review of blood culture contamination. Clin Microbiol Rev, 2006. 19(4): p. 788-802.

71. Surdulescu, S., D. Utamsingh, and R. Shekar, Phlebotomy teams reduce blood-culture contamination rate and save money. Clin Perform Qual Health Care, 1998. 6(2): p. 60-2. 
72. Weinbaum, F.I., et al., Doing it right the first time: quality improvement and the contaminant blood culture. J Clin Microbiol, 1997. 35(3): p. 563-5.

73. Martin, L., et al., Bartonella as a cause of blood culture-negative endocarditis. Description of five cases. Rev Esp Cardiol, 2009. 62(6): p. 694-7.

74. Trubiano, J.A., et al., Coxiella burnetii endocarditis after Q fever vaccination. J Med Microbiol, 2012. 61(Pt 12): p. 1775-9.

75. Glerant, J.C., et al., Utility of blood cultures in community-acquired pneumonia requiring hospitalization: influence of antibiotic treatment before admission. Respir Med, 1999. 93(3): p. 208-12.

76. Grace, C.J., et al., Usefulness of blood culture for hospitalized patients who are receiving antibiotic therapy. Clin Infect Dis, 2001. 32(11): p. 1651-5.

77. McKenzie, R. and L.G. Reimer, Effect of antimicrobials on blood cultures in endocarditis. Diagn Microbiol Infect Dis, 1987. 8(3): p. 165-72.

78. Zadoks, R.N. and J.L. Watts, Species identification of coagulase-negative staphylococci: genotyping is superior to phenotyping. Vet Microbiol, 2009. 134(1-2): p. 20-8.

79. van Veen, S.Q., E.C. Claas, and E.J. Kuijper, High-throughput identification of bacteria and yeast by matrix-assisted laser desorption ionization-time of flight mass spectrometry in conventional medical microbiology laboratories. J Clin Microbiol, 2010. 48(3): p. 900-7.

80. Anhalt, J.P. and C. Fenselau, Identification of bacteria using mass spectrometry. Anal. Chem, 1975. 47: p. 219-225.

81. Dubois, D., et al., Identification of a variety of Staphylococcus species by MALDI-TOF mass spectrometry. J Clin Microbiol, 2010. 48: p. 941-945.

82. Ferreira, L., et al., [Identifying bacteria using a matrix-assisted laser desorption ionization time-of-flight (MALDI-TOF) mass spectrometer. Comparison with routine methods used in clinical microbiology laboratories]. Enferm Infecc Microbiol Clin, 2010. 28(8): p. 492-7.

83. Bernardo, K., et al., Identification and discrimination of Staphylococcus aureus strains using matrix-assisted laser desorption/ionization-time of flight mass spectrometry. Proteomics, 2002. 2(6): p. 747-53.

84. Friedrichs, C., et al., Rapid identification of viridans streptococci by mass spectrometric discrimination. J Clin Microbiol, 2007. 45(8): p. 2392-7.

85. Degand, N., et al., Matrix-assisted laser desorption ionization-time of flight mass spectrometry for identification of nonfermenting gram-negative bacilli isolated from cystic fibrosis patients. $\mathrm{J}$ Clin Microbiol, 2008. 46(10): p. 3361-7.

86. Nagy, E., et al., The value of MALDI-TOF MS for the identification of clinically relevant anaerobic bacteria in routine laboratories. J Med Microbiol, 2012. 61(Pt 10): p. 1393-400.

87. Knoester, M., et al., Routine identification of clinical isolates of anaerobic bacteria: matrixassisted laser desorption ionization-time of flight mass spectrometry performs better than conventional identification methods. J Clin Microbiol, 2012. 50(4): p. 1504.

88. Marklein, G., et al., MALDI - TOF Mass-Spectrometry for Fast and Reliable Identification of Clinical Yeast Isolates. J Clin Microbiol, 2009.

89. Christner, M., et al., Rapid identification of bacteria from positive blood culture bottles by use of matrix-assisted laser desorption-ionization time of flight mass spectrometry fingerprinting. J Clin Microbiol, 2010. 48(5): p. 1584-91.

90. La Scola, B. and D. Raoult, Direct identification of bacteria in positive blood culture bottles by matrix-assisted laser desorption ionisation time-of-flight mass spectrometry. PLoS One, 2009. 4(11): p. e8041.

91. Prod'hom, G., et al., Matrix-assisted laser desorption ionization-time of flight mass spectrometry for direct bacterial identification from positive blood culture pellets. J Clin Microbiol, 2010. 48(4): p. 1481-3.

92. Edwards-Jones, V., et al., Rapid discrimination between methicillin-sensitive and methicillinresistant Staphylococcus aureus by intact cell mass spectrometry. J Med Microbiol, 2000. 49(3): p. 295-300. 
93. Walker, J., et al., Intact cell mass spectrometry (ICMS) used to type methicillin-resistant Staphylococcus aureus: media effects and inter-laboratory reproducibility. J Microbiol Methods, 2002. 48(2-3): p. 117-26.

94. Schaumann, R., et al., A step towards the discrimination of beta-lactamase-producing clinical isolates of Enterobacteriaceae and Pseudomonas aeruginosa by MALDI-TOF mass spectrometry. Med Sci Monit, 2012. 18(9): p. MT71-7.

95. Vlek, A.L., M.J. Bonten, and C.H. Boel, Direct matrix-assisted laser desorption ionization timeof-flight mass spectrometry improves appropriateness of antibiotic treatment of bacteremia. PLoS One, 2012. 7(3): p. e32589.

96. Hartmann, H., et al., Rapid identification of Staphylococcus aureus in blood cultures by a combination of fluorescence in situ hybridization using peptide nucleic acid probes and flow cytometry. J Clin Microbiol, 2005. 43(9): p. 4855-7.

97. Wang, P., Simultaneous detection and differentiation of Staphylococcus species in blood cultures using fluorescence in situ hybridization. Med Princ Pract, 2010. 19(3): p. 218-21.

98. Gescher, D.M., et al., Fluorescence in situ hybridisation (FISH) accelerates identification of Gram-positive cocci in positive blood cultures. Int J Antimicrob Agents, 2008.

99. Jansen, G.J., et al., Rapid identification of bacteria in blood cultures by using fluorescently labeled oligonucleotide probes. J Clin Microbiol, 2000. 38(2): p. 814-7.

100. Sogaard, M., H. Stender, and H.C. Schonheyder, Direct identification of major blood culture pathogens, including Pseudomonas aeruginosa and Escherichia coli, by a panel of fluorescence in situ hybridization assays using peptide nucleic acid probes. J Clin Microbiol, 2005. 43(4): p. 1947-9.

101. Wellinghausen, N., et al., Rapid detection of Brucella spp. in blood cultures by fluorescence in situ hybridization. J Clin Microbiol, 2006. 44(5): p. 1828-30.

102. Kempf, V.A., K. Trebesius, and I.B. Autenrieth, Fluorescent In situ hybridization allows rapid identification of microorganisms in blood cultures. J Clin Microbiol, 2000. 38(2): p. 830-8.

103. Peters, R.P., et al., Rapid identification of pathogens in blood cultures with a modified fluorescence in situ hybridization assay. J Clin Microbiol, 2006. 44(11): p. 4186-8.

104. Mullis, K.B. and F.A. Faloona, Specific synthesis of DNA in vitro via a polymerase-catalyzed chain reaction. Methods Enzymol, 1987. 155: p. 335-50.

105. Huang, Q. and W.L. Fu, Comparative analysis of the DNA staining efficiencies of different fluorescent dyes in preparative agarose gel electrophoresis. Clin Chem Lab Med, 2005. 43(8): p. 841-2.

106. Tissari, P., et al., Accurate and rapid identification of bacterial species from positive blood cultures with a DNA-based microarray platform: an observational study. Lancet, 2010. 375(9710): p. 224-30.

107. Sanger, F., S. Nicklen, and A.R. Coulson, DNA sequencing with chain-terminating inhibitors. Proc Natl Acad Sci U S A, 1977. 74(12): p. 5463-7.

108. Smith, L.M., et al., Fluorescence detection in automated DNA sequence analysis. Nature, 1986. 321(6071): p. 674-9.

109. Espy, M.J., et al., Real-time PCR in clinical microbiology: applications for routine laboratory testing. Clin Microbiol Rev, 2006. 19(1): p. 165-256.

110. Mackay, I.M., Real-time PCR in the microbiology laboratory. Clin Microbiol Infect, 2004. 10(3): p. 190-212.

111. leven, M. and H. Goossens, Relevance of nucleic acid amplification techniques for diagnosis of respiratory tract infections in the clinical laboratory. Clin Microbiol Rev, 1997. 10(2): p. 24256.

112. Persing, D.H., Diagnostic molecular microbiology. Current challenges and future directions. Diagn Microbiol Infect Dis, 1993. 16(2): p. 159-63.

113. Greisen, K., et al., PCR primers and probes for the 16S rRNA gene of most species of pathogenic bacteria, including bacteria found in cerebrospinal fluid. J Clin Microbiol, 1994. 32(2): p. 335-51.

114. Jou, N.T., et al., Single-tube, nested, reverse transcriptase PCR for detection of viable Mycobacterium tuberculosis. J Clin Microbiol, 1997. 35(5): p. 1161-5. 
115. Klausegger, A., et al., Gram type-specific broad-range PCR amplification for rapid detection of 62 pathogenic bacteria. J Clin Microbiol, 1999. 37(2): p. 464-6.

116. Van Burik, J.A., et al., Panfungal PCR assay for detection of fungal infection in human blood specimens. J Clin Microbiol, 1998. 36(5): p. 1169-75.

117. Brakstad, O.G., K. Aasbakk, and J.A. Maeland, Detection of Staphylococcus aureus by polymerase chain reaction amplification of the nuc gene. J Clin Microbiol, 1992. 30(7): p. $1654-60$.

118. Costa, A.M., I. Kay, and S. Palladino, Rapid detection of mecA and nuc genes in staphylococci by real-time multiplex polymerase chain reaction. Diagn Microbiol Infect Dis, 2005.

51(1): p. 13-7.

119. Iwase, T., et al., Rapid identification and specific quantification of Staphylococcus epidermidis by 5' nuclease real-time polymerase chain reaction with a minor groove binder probe. Diagn Microbiol Infect Dis, 2008. 60(2): p. 217-9.

120. Martineau, F., et al., Development of a PCR assay for identification of staphylococci at genus and species levels. J Clin Microbiol, 2001. 39(7): p. 2541-7.

121. Prere, M.F., et al., Genotype MRSA, a new genetic test for the rapid identification of staphylococci and detection of mecA gene. Pathol Biol (Paris), 2006. 54(8-9): p. 502-5.

122. Wellinghausen, N., et al., Rapid detection of Staphylococcus aureus bacteremia and methicillin resistance by real-time PCR in whole blood samples. Eur J Clin Microbiol Infect Dis, 2009. 28(8): p. 1001-5.

123. Hassan-King, M., et al., Detection of Streptococcus pneumoniae DNA in blood cultures by PCR. J Clin Microbiol, 1994. 32(7): p. 1721-4.

124. Corless, C.E., et al., Simultaneous detection of Neisseria meningitidis, Haemophilus influenzae, and Streptococcus pneumoniae in suspected cases of meningitis and septicemia using real-time PCR. J Clin Microbiol, 2001. 39(4): p. 1553-8.

125. Peters, R.P., et al., Quantitative detection of Staphylococcus aureus and Enterococcus faecalis DNA in blood to diagnose bacteremia in patients in the intensive care unit. J Clin Microbiol, 2007. 45(11): p. 3641-6.

126. Loeffler, J., et al., Nucleic acid sequence-based amplification of Aspergillus RNA in blood samples. J Clin Microbiol, 2001. 39(4): p. 1626-9.

127. Wellinghausen, N., et al., Rapid diagnosis of candidaemia by real-time PCR detection of Candida DNA in blood samples. J Med Microbiol, 2009. 58(Pt 8): p. 1106-11.

128. Mancini, N., et al., The era of molecular and other non-culture-based methods in diagnosis of sepsis. Clin Microbiol Rev, 2010. 23(1): p. 235-51.

129. Dierkes, C., et al., Clinical impact of a commercially available multiplex PCR system for rapid detection of pathogens in patients with presumed sepsis. BMC Infect Dis, 2009. 9: p. 126.

130. Hansen, W.L., et al., Molecular probes for diagnosis of clinically relevant bacterial infections in blood cultures. J Clin Microbiol, 2010. 48(12): p. 4432-8.

131. Barken, K.B., J.A. Haagensen, and T. Tolker-Nielsen, Advances in nucleic acid-based diagnostics of bacterial infections. Clin Chim Acta, 2007. 384(1-2): p. 1-11.

132. Fredricks, D.N. and D.A. Relman, Improved amplification of microbial DNA from blood cultures by removal of the PCR inhibitor sodium polyanetholesulfonate. J Clin Microbiol, 1998. 36(10): p. 2810-6.

133. Klouche, M. and U. Schroder, Rapid methods for diagnosis of bloodstream infections. Clin Chem Lab Med, 2008. 46(7): p. 888-908.

134. Song, J.H., et al., Detection of Salmonella typhi in the blood of patients with typhoid fever by polymerase chain reaction. J Clin Microbiol, 1993. 31(6): p. 1439-43.

135. Iralu, J.V., et al., Diagnosis of Mycobacterium avium bacteremia by polymerase chain reaction. J Clin Microbiol, 1993. 31(7): p. 1811-4.

136. Rantakokko-Jalava, K. and J. Jalava, Optimal DNA isolation method for detection of bacteria in clinical specimens by broad-range PCR. J Clin Microbiol, 2002. 40(11): p. 4211-7.

137. Handschur, M., et al., Preanalytic removal of human DNA eliminates false signals in general $16 S$ rDNA PCR monitoring of bacterial pathogens in blood. Comp Immunol Microbiol Infect Dis, 2008. 
138. Al-Soud, W.A. and P. Radstrom, Purification and characterization of PCR-inhibitory components in blood cells. J Clin Microbiol, 2001. 39(2): p. 485-93.

139. Muhl, $\mathrm{H}$. , et al., Activity and DNA contamination of commercial polymerase chain reaction reagents for the universal $16 S$ rDNA real-time polymerase chain reaction detection of bacterial pathogens in blood. Diagn Microbiol Infect Dis, 2010. 66(1): p. 41-9.

140. Corless, C.E., et al., Contamination and sensitivity issues with a real-time universal 16S rRNA PCR. J Clin Microbiol, 2000. 38(5): p. 1747-52.

141. Horz, H.P., et al., Selective isolation of bacterial DNA from human clinical specimens. J Microbiol Methods, 2008. 72(1): p. 98-102.

142. Horz, H.P., et al., New methods for selective isolation of bacterial DNA from human clinical specimens. Anaerobe, 2010. 16(1): p. 47-53.

143. Wiesinger-Mayr, $\mathrm{H}$., et al., Establishment of a semi-automated pathogen DNA isolation from whole blood and comparison with commercially available kits. J Microbiol Methods, 2011. 85(3): p. 206-13.

144. Gebert, S., D. Siegel, and N. Wellinghausen, Rapid detection of pathogens in blood culture bottles by real-time PCR in conjunction with the pre-analytic tool MolYsis. J Infect, 2008. 57: p. 307-316.

145. Smith, M., et al., An In-Solution Hybridisation Method for the Isolation of Pathogen DNA from Human DNA-rich Clinical Samples for Analysis by NGS. The Open Genomics Journal, 2012. 5: p. 18-29.

146. Casalta, J.P., et al., Evaluation of the LightCycler(R) SeptiFast test in the rapid etiologic diagnostic of infectious endocarditis. Eur J Clin Microbiol Infect Dis, 2009. 28(6): p. 569-73.

147. Mauro, M.V., et al., Diagnostic utility of LightCycler SeptiFast and procalcitonin assays in the diagnosis of bloodstream infection in immunocompromised patients. Diagn Microbiol Infect Dis, 2012. 73(4): p. 308-11.

148. Kuhn, C., et al., Evaluation of commercial universal rRNA gene PCR plus sequencing tests for identification of bacteria and fungi associated with infectious endocarditis. J Clin Microbiol, 2011. 49(8): p. 2919-23.

149. Wellinghausen, N., et al., Diagnosis of bacteremia in whole-blood samples by use of a commercial universal 16S rRNA gene-based PCR and sequence analysis. J Clin Microbiol, 2009. 47(9): p. 2759-65.

150. Fitting, C., et al., DNAemia Detection by Multiplex PCR and Biomarkers for Infection in Systemic Inflammatory Response Syndrome Patients. PLoS One, 2012. 7(6): p. e38916.

151. Serra, J., et al., Clinical evaluation of the Magicplex Sepsis Real-time Test (Seegene) to detect Candida DNA in pediatric patients. Crit Care, 2012. 16: p. 21.

152. Oliver, J.D., The viable but nonculturable state in bacteria. J Microbiol, 2005. 43 Spec No: p. 93-100.

153. Birch, L., et al., A comparison of nucleic acid amplification techniques for the assessment of bacterial viability. Lett Appl Microbiol, 2001. 33(4): p. 296-301.

154. Aellen, S., et al., Detection of live and antibiotic-killed bacteria by quantitative real-time PCR of specific fragments of rRNA. Antimicrob Agents Chemother, 2006. 50(6): p. 1913-20.

155. Morre, S.A., et al., Monitoring of Chlamydia trachomatis infections after antibiotic treatment using RNA detection by nucleic acid sequence based amplification. Mol Pathol, 1998. 51(3): p. 149-54.

156. Lindahl, T., Instability and decay of the primary structure of DNA. Nature, 1993. 362(6422): p. 709-15.

157. Chuang, Y.C., S.C. Chang, and W.K. Wang, Using the rate of bacterial clearance determined by real-time polymerase chain reaction as a timely surrogate marker to evaluate the appropriateness of antibiotic usage in critical patients with Acinetobacter baumannii bacteremia. Crit Care Med, 2012. 40(8): p. 2273-80.

158. Sakka, S.G., et al., Blood infection diagnosis by $16 \mathrm{~S}$ rDNA broad-spectrum polymerase chain reaction: the relationship between antibiotic treatment and bacterial DNA load. Anesth Analg, 2009. 109(5): p. 1707-8. 
159. Peters, R.P., et al., Streptococcus pneumoniae DNA load in blood as a marker of infection in patients with community-acquired pneumonia. J Clin Microbiol, 2009. 47(10): p. 3308-12.

160. Nocker, A., et al., Use of propidium monoazide for live/dead distinction in microbial ecology. Appl Environ Microbiol, 2007. 73(16): p. 5111-7.

161. Josephson, K.L., C.P. Gerba, and I.L. Pepper, Polymerase chain reaction detection of nonviable bacterial pathogens. Appl Environ Microbiol, 1993. 59(10): p. 3513-5.

162. Sheridan, G.E., et al., Detection of $m R N A$ by reverse transcription-PCR as an indicator of viability in Escherichia coli cells. Appl Environ Microbiol, 1998. 64(4): p. 1313-8.

163. Hellyer, T.J., et al., Detection of viable Mycobacterium tuberculosis by reverse transcriptasestrand displacement amplification of mRNA. J Clin Microbiol, 1999. 37(3): p. 518-23. 



\section{Chapter 2}

Development and clinical evaluation of a tuf gene based realtime PCR for Gram positive cocci: a study using blood cultures and whole blood

PAMM Laboratory, Department of Medical Microbiology, Eindhoven/Veldhoven, The Netherlands ${ }_{2}^{2}$ Fontys University of Applied Science, Department of Medical Molecular Diagnostics, Eindhoven, The Netherlands ${ }^{3}$ Maastricht University Medical Centre, CAPHRI, Department of Medical Microbiology, Maastricht, The Netherlands ${ }^{4}$ Catharina Hospital, Department of Intensive Care, Eindhoven, The Netherlands

5 Jeroen Bosch Hospital, Department of Molecular Diagnostics, 's-Hertogenbosch, The Netherlands 8

Submitted 


\section{Abstract}

Bloodstream infections (BSI) are often nosocomial infections that are frequently caused by Gram-positive cocci and are associated with high morbidity and mortality rates. Although culture techniques remain the gold standard for pathogen identification they are hampered by the lengthy time-to-result. To accelerate the diagnostic procedure, two multiplex real-time PCRs were developed to detect these pathogens.

The PCRs were evaluated using 100 bacterial strains to determine the specificity. Additionally, 317 blood cultures and 30 whole blood samples were analysed, to study the potential clinical value of this multiplex assay. Five bacterial DNA isolation methods were compared to determine the optimal sensitivity of this assay on remnant whole blood samples.

The analytical sensitivity of this real-time PCR is 0.4 CFU for Staphylococcus aureus and $4 \mathrm{CFU}$ for Enterococcus faecalis. Both the S. aureus and the Enterococcus spp. probes indicated to be $100 \%$ sensitive and specific. The PCRs showed $100 \%$ sensitivity and specificity on positive blood culture bottles containing Gram-positive cocci. The optimal DNA detection limit from $1 \mathrm{ml}$ whole blood was $10 \mathrm{CFU} / \mathrm{ml}$, obtained with the MolYsis Complete kit. This method was therefore used on $1 \mathrm{ml}$ EDTA blood of 30 patients suspected of having BSI, to show proof-of-principle. Of the whole blood samples analysed, identical results between culture and PCR were found in $81.8 \%(18 / 22)$ of the cases. All CoNS and S. aureus samples were confirmed with PCR.

In conclusion, sensitive and specific real-time PCR assays were developed enabling accurate and fast detection of pathogens from clinical samples indicative for Gram-positive cocci. The pilot study on remaining EDTA blood of suspected sepsis patients supports further evaluation in a larger patient group. 


\section{Introduction}

Bloodstream infection (BSI) is a serious medical condition. Gram-positive microorganisms, especially Staphylococcus aureus, coagulase-negative staphylococci (CoNS), and Enterococcus species, account for the majority of episodes of bacteremia in critically ill patients in the intensive care unit (ICU) [1, 2]. CoNS are increasingly recognized as causative agents for both community-acquired and nosocomial bloodstream infections worldwide, mainly due to increased usage of medical devices [3]. A rapid and reliable method to detect these pathogens is therefore of clinical importance. Early detection and subsequent adequate treatment of bloodstream infections is critical for patient outcomes [4].

Blood cultures are still considered as the gold standard for pathogen detection from blood. However, this technique is slow, taking 24-72 hours, and can result in false-negative results $(30 \%)$ even when the patient shows clear signs of systemic infection [5]. This is frequently thought to be the result of previous administration of antibiotics [1].

Nucleic acid based techniques offer a rapid and sensitive option for detecting pathogens directly from clinical specimens [6-11]. However, blood consists of many cells containing genomic DNA. This highly abundant genomic DNA might decrease the sensitivity of a PCR specifically detecting bacterial DNA. An optimal sample processing method should diminish human DNA, concentrate pathogen DNA and wash out inhibitory factors present in clinical samples. Several studies have investigated both automated and manual methods for isolation of bacterial DNA from several clinical specimens [12-16]. Sample preparation is of crucial importance when a sensitive test result needs to be obtained.

This study aims to develop a fast and sensitive PCR to detect $S$. aureus, CoNS, and Enterococcus spp, based on the tuf gene, which has been described as a reliable target for detection of staphylococci $[17,18]$. Furthermore, this assay will be clinically evaluated using blood culture materials and whole blood specimens of sepsis suspected patients, using a combined sensitive pathogen DNA isolation method and in-house PCR.

\section{Materials and Methods}

\section{Bacterial strains}

Bacterial strains $(n=100)$, commonly encountered in our laboratory were selected (Table 1$)$. The bacteria tested were mainly clinical isolates $(n=95)$, although a few reference strains (ATCC 33591 MRSA, ATCC 25923 MSSA, ATCC 12228 S. epidermidis, RIVM 122 S. hominis, and ATCC 29212 E. faecalis) were used (derived from the American Type Culture Collection and the National Institute for Health and Environment (RIVM, Bilthoven, The Netherlands)). All clinical isolates were identified by standard culture and biochemical methods used in our certified diagnostic laboratory (PAMM laboratories, Veldhoven, The Netherlands). 
Table 1. Bacterial strains used for specificity of the tuf real-time PCR assay.

\begin{tabular}{|c|c|c|c|c|}
\hline Strains & $\mathbf{n}$ & Staphylococcus probe & S.aureus Probe & Enterococcus Probe \\
\hline S.aureus & 22 & 22 & 22 & 0 \\
\hline $\begin{array}{l}\text { CoNS: S.epidermidis (4), S.haemolyticus } \\
\text { (2), S.hominis (1), S.schleiferi (1), } \\
\text { S.lugdunensis (2), S.arlettae (1), S.sciuri } \\
\text { (1), S.auricularis (1), S.capitis (1), } \\
\text { S.simulans (1), S. saprofyticus (1), } \\
\text { S.cohnii (1), S. warneri (1) }\end{array}$ & 18 & 18 & 0 & 0 \\
\hline $\begin{array}{l}\text { Non-staphylococcus: E.coli (3), } \\
\text { C.albicans (1), Hemolytical Strep A (1), } \\
\text { Hemolytical Strep B (3), S. marcescens } \\
\text { (1), M.morgani (1), P. aeruginosa (1), } \\
\text { K.plantico (1), Enterobacter spp (1), } \\
\text { Yeast (2), Difteroides (1), P.vulgaris (2), } \\
\text { S.sonnei (1), Y. enterocolitica (2), K. } \\
\text { pneumoniae (3), B. cereus (2), S. mutans } \\
\text { (1), S. pneumoniae (2), E. cloacae (2), } \\
\text { Lactobacillus spp (1), L. acidophilus (1), } \\
\text { S. flexneri type } 6 \text { (1), S. enteritidis (1), M. } \\
\text { catarrhalis (2), S. typhimurium (1), S. } \\
\text { paratyphi (1), S. bovis (1), S. pyogenes } \\
\text { (2), L. monocytogenes (2), } \\
\text { Campylobacter spp (2), Bacteroides spp } \\
\text { (3), H. influenzae (2), V. cholerae (2) }\end{array}$ & 53 & L. monocytogenes & 0 & 0 \\
\hline E.Faecalis (4) and E.faecium (3) & 7 & 7 & 0 & 7 \\
\hline
\end{tabular}

\section{Clinical samples}

Three hundred and seventeen positive blood cultures were used, which consisted of BacT/ ALERT Standard Aerobe (SA) and Standard Anaerobe (SN) bottles (bioMérieux, Marcy L'Etoile, France). Bottles were handled according to manufacturer's instructions. After being flagged positive by the BacT/ALERT 3D system (bioMérieux), Gram staining was performed. Positive blood cultures containing either Gram-positive cocci or Gram-negatives were included in the study. All positive blood cultures were further evaluated by routine culture and biochemical methods. The negative culture bottles had been incubated for 1 week and remained negative before inclusion in this study.

Whole blood was obtained from patients admitted to the Intensive Care Unit (ICU) of the Catharina Hospital, Eindhoven, The Netherlands. Patients were included in the study when showing $\geq 2$ signs of systemic inflammatory response syndrome (SIRS) and were suspected of having a bloodstream infection [19]. Duration of hospital stay was not taken into account. Patients $(n=30)$ were selected by the intensive care physician. For routine diagnostic purposes both blood cultures $(n=22)$ for pathogen detection and EDTA whole blood were collected. After routine diagnostics, $1 \mathrm{ml}$ remnant whole blood of these patients was obtained after blood culture was drawn. Whole blood was used for pathogen DNA isolation. Laboratory and clinical data were anonimised for analyses and no informant consent was obtained. This 
is in agreement with the code for proper use of human tissue as formulated by the Dutch Federation of Medical Scientific Societies.

\section{DNA isolation}

DNA from bacterial strains was obtained by 10 min boiling of $1 \mathrm{McF}$ bacterial suspensions in Tris-HCL $20 \mathrm{mM} \mathrm{pH}$ 8.3. Blood culture DNA was prepared by diluting positive blood culture material 1:100 in Tris- $\mathrm{HCl} 20 \mathrm{mM} \mathrm{pH}$ 8.3. This was centrifuged for $5 \mathrm{~min}$ at maximum speed and the pellet was subsequently dissolved in $100 \mu \mathrm{l}$ aquadest. When the DNA sample of blood culture material showed to be red/brownish of colour, it was additionally diluted 10 fold before real-time PCR analysis.

\section{EDTA whole blood sample preparation}

For spiking experiments, randomly taken EDTA blood (remaining after diagnostics) of the same blood type (i.e. $\mathrm{O}^{+}$), was pooled and subsequently spiked with a $2 \mathrm{McF}$ MRSA (ATCC 33591), to obtain a ten-fold dilution series. Bacterial DNA was isolated with 5 different methods: EasyMAG generic protocol, EasyMAG specific A off-board lysis protocol, EasyMAG specific B off-board lysis protocol (bioMérieux, Marcy L'Etoile, France), MolYsis Complete kit (1 and $5 \mathrm{ml}$ ) according to manufacturer's instructions (Molzym $\mathrm{GmbH}$, Bremen, Germany), and the MolYsis Basic kit (Molzym GmbH, Bremen, Germany) followed by the EasyMAG spec B protocol. Input for each isolation method was $200 \mu \mathrm{l}$, except for the Molzym Complete kit isolations for which the recommended volumes of $1 \mathrm{ml}$ and $5 \mathrm{ml}$ were used. Samples isolated with one of the EasyMAG protocols were eluted in $70 \mu$ l elution buffer provided; all other samples were eluted in $100 \mu$ l elution buffer provided with the isolation system. From each sample of the dilution series, $100 \mu \mathrm{l}$ was plated on bloodagar to determine CFU/ml. DNA samples were analysed with the tuf LightCycler assay described below. Each isolation was at least performed in three independent experiments. Of the EDTA blood samples derived from sepsis suspected patients $1 \mathrm{ml}$ was processed with the best performing protocol (see Results section).

\section{Tuf real-time PCR assay}

Two multiplex real-time PCRs were developed to detect S. aureus, CoNS, and Enterococcus spp., as schematically shown in Figure 1. PCR 1 detects and differentiates both Staphylococcus spp. and S. aureus specifically, and PCR 2 detects both Enterococcus spp. and Phocine herpesvirus ( $\mathrm{PhHV}, \mathrm{gB}$ polymerase), which is used as an internal amplification control and to check for inhibitory factors.

Primers and probes are described in Table 2. All Staphylococcus spp. and S. aureus primers and probe positions are based on $S$. aureus tuf gene sequence (GenBank accession no AF298796). Enterococcus probe position is based on E. faecium and E. faecalis tuf gene 


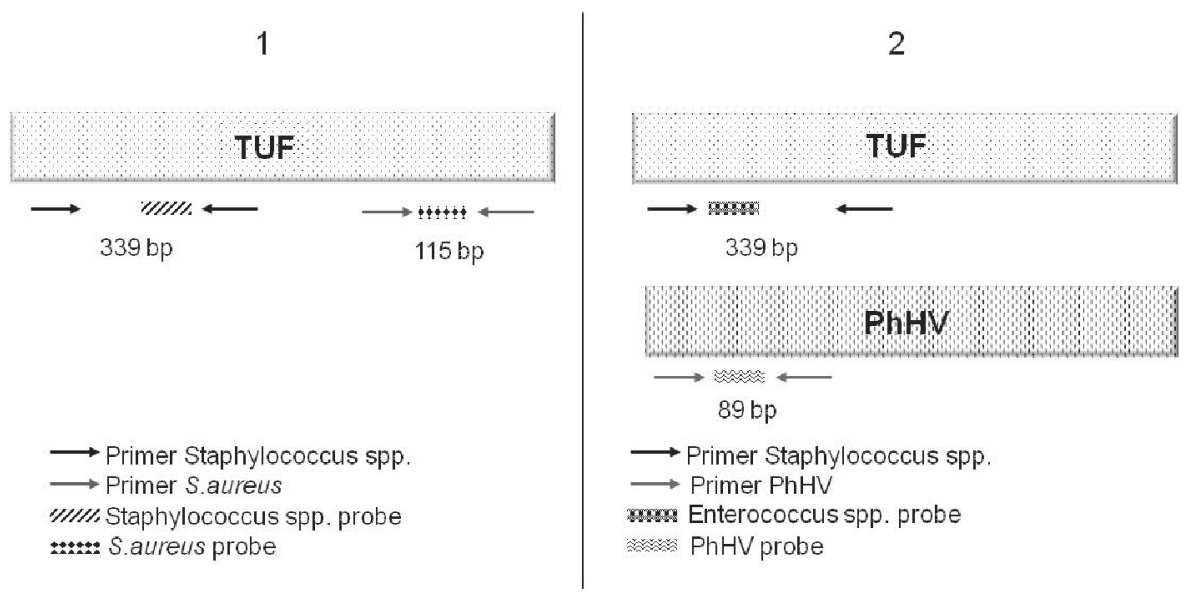

Figure 1. Schematic overview of tuf real-time PCR duplex assays.

Two real-time PCR duplex tests are performed on each sample. By using duplex 1 (left), two fragments of the tuf gene are amplified detecting both Staphylococcus spp. (339bp) and S. aureus (115bp) specifically. Duplex 2 is used to detect Enterococcus spp, based on the tuf gene, and PhHV (gB polymerase) which is added to each eluate as amplification and inhibition control. The figure is not presented on correct scale.

Table 2. Primers and probes used for tuf real-time PCR assays.

\begin{tabular}{llll}
\hline Primers and Probes & Sequence 5'-3' & Position & Reference \\
\hline Staphylococcus FW & ccaatgccacaaactcgtga & $32-51$ & {$[25]$} \\
Staphylococcus RV & cacgaccagtgattgagaatacg & $371-349$ & {$[25]$} \\
Staphylococcus probe & VIC-ccattcatgatgccagttg-BHQ1 & $326-344$ & {$[25]$} \\
S. aureus FW & tcctggttcaattacaccacatactg & $583-608$ & {$[25]$} \\
S. aureus RV & ggaaatagaattgtggacgatagtttga & $698-671$ & {$[25]$} \\
S. aureus probe & FAM-tgataatacrtawacttctgc-BHQ1 & $637-617$ & This study \\
Enterococcus probe & FAM-gctgcagttgacgaata-BHQ1 & $281-297$ & This study \\
PhHV-1 FW & gggcgaatcacagattgaatc & & {$[20]$} \\
PhHV-1 RV & gcggttccaaacgtaccaa & & {$[20]$} \\
PhHV-1 probe & VIC-ttttatgtgtccgccaccatctggatc-BHQ1 & & {$[20]$} \\
\hline
\end{tabular}

sequences (GenBank accession no AF124222 and no AF124221). Oligonucleotide primers were obtained from Eurogentec (Liège, Belgium). XS probes were obtained from Biolegio (Nijmegen, The Netherlands). PhHV-1 primers and VIC labeled probe [20] were obtained from Applied Biosystems (Nieuwerkerk aan den IJssel, The Netherlands). In silico analysis (BLAST) of the probes revealed cross-reactivity of the Staphylococcus spp. probe with Enterococcus spp, and the Enterococcus spp. probe detects besides E. faecalis and 
E. faecium 9 other Enterococcus spp. (E. saccharolyticus, E. mundtii, E. hirae, E. urans, E. dispar, E. columbae, E. cecorum, E. casseliflavus, E. qallinarum).

PCR mixtures consisted of 5x LightCycler TaqMan Master mixture (Roche Diagnostics, Almere, The Netherlands), $0.9 \mu \mathrm{M}$ concentration of each primer, $0.2 \mu \mathrm{M}$ concentration of each probe, and aquadest in a volume of $15 \mu$ to which $5 \mu$ I DNA sample was added, leading to a final reaction volume of $20 \mu \mathrm{l}$ in each capillary. Primers and probe targeting PhHV had a concentration of $0.1 \mu \mathrm{M}$ each. All samples were amplified on the LightCycler v2.0 (Roche Diagnostics) according to the following cycling parameters: an initial incubation step for $10 \mathrm{~min}$ at $95^{\circ} \mathrm{C}$, subsequently $45 \mathrm{cycli}$ of $15 \mathrm{sec} 95^{\circ} \mathrm{C}$ followed by $1 \mathrm{~min} 60^{\circ} \mathrm{C}$. Results were analyzed using LightCycler Software version 4.05 (Roche Diagnostics).

\section{Results}

\section{Analytical specificity of tuf real-time PCR}

Specificity of the assay was determined using a representative panel of bacteria (Table 1). The $S$. aureus and Enterococcus spp. probe showed correct results in the tuf real-time PCR assay with all tested bacterial strains. The Staphylococcus spp. probe showed crosshybridisation with E. faecalis, E. faecium, and L. monocytogenes, although in silico analysis indicated at least 1 to 3 mismatches with the primers.

\section{Analytical sensitivity of tuf real-time PCR}

The analytical sensitivity of the tuf real-time PCR was determined using 10-fold serial dilutions in Tris- $\mathrm{HCl}$ buffer in combination with MolYsis Complete DNA isolation (Molzym), and was shown to be $0.4 \mathrm{CFU}$ per PCR reaction for $S$. aureus and 4 CFU per PCR for E. faecalis. Additionally, the sensitivity of the tuf PCR was analysed on serial dilutions of $S$. aureus spiked EDTA whole blood samples. From whole blood bacterial DNA was isolated according to 5 different methods as described in Materials and Methods (Table 3). All isolations were performed in 3 independent experiments. The automated extractor EasyMAG did not result in sensitive detection from whole blood material. The generic protocol resulted in a detection limit of $10^{5} \mathrm{CFU} / \mathrm{ml}$, however in one run $10^{4} \mathrm{CFU} / \mathrm{ml}$ was detected. The most sensitive detection limit using the EasyMAG was $10^{3} \mathrm{CFU} / \mathrm{ml}$; this was reached by using the off-board lysis combined with the specific B protocol. Bacterial DNA isolation with the MolYsis Complete kit (Molzym) resulted in the most sensitive detection of $10 \mathrm{CFU} / \mathrm{ml}$ for $S$. aureus from $1 \mathrm{ml}$ EDTA blood. This equals a sensitivity of 0.5 CFU/PCR. Occasionally, $1 \mathrm{CFU} / \mathrm{ml}$ could be detected when bacterial DNA was isolated from $5 \mathrm{ml}$ spiked EDTA blood with the MolYsis Complete kit (1 out of 3 isolations). Inhibiting factors were not present since the internal control, i.e. PhHV gave comparable signals $(\mathrm{Cp} \pm 0.5)$ for all samples in one run. 
Table 3. Evaluation of 5 different DNA isolation protocols on EDTA whole blood spiked with 10 -fold dilution series of Staphylococcus aureus.

\begin{tabular}{lcccccc}
\hline & \multicolumn{7}{c}{ Detection limit in colony forming units/ ml } \\
DNA isolation protocol & $10^{5}$ & $\mathbf{1 0}$ & $\mathbf{1 . 0 0 0}$ & $\mathbf{1 0 0}$ & $\mathbf{1 0}$ & $\mathbf{1}$ \\
\hline EasyMAG generic & + & + & - & - & - & - \\
EasyMAG specific A & + & + & - & - & - & - \\
EasyMAG specific B & + & + & + & - & - & - \\
MolYsis Basic + EasyMAG specific B & + & + & + & + & - & - \\
MolYsis Complete 1 ml & + & + & + & + & + & - \\
MolYsis Complete 5 ml & + & + & + & + & + & \pm \\
\hline
\end{tabular}

+ detected; - not detected; \pm detected in 1 of 3 experiments (in tuf real-time PCR)

\section{Clinical evaluation}

\section{Blood cultures}

The tuf assay was clinically evaluated on 317 blood cultures which were either culture negative or showed presence of Gram-positive cocci or Gram-negative bacteria (Table 4). Conventional culture showed that 79 bottles contained $S$. aureus, 78 bottles CoNS, 5 bottles showed Micrococcus spp., 22 bottles Enterococcus spp. (E. faecalis or E. faecium), 97 bottles indicated Gram negatives to be present, 30 bottles were culture negative and 6 bottles contained double infections. All 79 blood cultures containing $S$. aureus were correctly identified by this PCR assay. CoNS containing bottles correctly only showed a positive signal with the Staphylococcus spp. probe. Bottles containing Micrococcus spp. were all negative with this assay, and the Enterococcus spp. were all positive with the Enterococcus spp. probe. As described before, Enterococcus spp. also showed a false positive signal with the Staphylococcus spp. probe. All double infections found $(n=6)$ during this study were correctly identified by PCR. In addition to the microorganisms detected by culture, the tuf assay detected CoNS in $13 / 97$ Gram-negative bottles (13.4\%), and in 5/30 culture negative bottles (16.6\%). In 5/13 Gram-negative bottles, which showed the unexpected presence of CoNS by PCR, a high load was detected as $\mathrm{Cp}$ values of less than 36 were found. In the culture negative bottles the CoNS detected by PCR showed a Cp value higher than 40, and this was also found for 8/13 Gram-negative bottles that indicated presence of CoNS ( $\mathrm{Cp}>39)$. The PhHV signals were comparable for all samples, as well as the negative (water) control, tested in one run $(\mathrm{Cp} \pm 0.5)$.

\section{EDTA blood samples}

In addition to blood cultures, whole blood samples from a total of 30 patients with suspected bloodstream infection were evaluated in a small proof-of-principle pilot study. The MolYsis Complete kit was used to isolate bacterial DNA from $1 \mathrm{ml}$ residual EDTA blood after completion of all other routine diagnostics. Available blood cultures data of these patients 
Table 4. Comparison of blood culture results with tuf real-time PCR.

\begin{tabular}{lllll}
\hline & \multicolumn{4}{c}{ tuf real-time PCR } \\
Blood culture results & & $\begin{array}{l}\text { S. aureus } \\
\text { probe }\end{array}$ & $\begin{array}{l}\text { Staphylococcus } \\
\text { probe }\end{array}$ & $\begin{array}{l}\text { Enterococcus } \\
\text { probe }\end{array}$ \\
\hline S. aureus & 79 & 79 & 79 & - \\
CoNS & 78 & - & 78 & - \\
Micrococcus spp. & 5 & - & - & - \\
Enterococcus spp. & 22 & - & 22 & 22 \\
Enterococcus spp. + S. aureus & 3 & 3 & 3 & 3 \\
Gram negative culture & 97 & - & $13(8 / 13 \mathrm{Cp}>39 ;$ & - \\
& & & $5 / 13 \mathrm{Cp}<36)$ & - \\
Gram negative + CoNS & 1 & - & $1(\mathrm{Cp}<24)$ & $2(\mathrm{Cp}<29)$ \\
Gram negative + Enterococcus spp. & 2 & - & $2(\mathrm{Cp}<29)$ & - \\
Negative blood culture & 30 & - & $5(\mathrm{Cp}>40)$ & \\
Total & 317 & & & \\
\hline
\end{tabular}

CoNS: coagulase-negative Staphylococci; - indicates negative test result

were compared to tuf real-time PCR data. Of 22/30 patients it was possible to compare PCR data to blood cultures results (Table 5). From 8/30 patients no blood cultures were drawn. In 18 out of the 22 samples $(81.8 \%)$ of which blood culture and PCR data were available, identical results were found. Fourteen culture negative samples were also negative in PCR, whereas S. aureus (sample 5 and 26 ) and CoNS (sample 1) positivity was confirmed in PCR. In 4/22 (18.2\%) of the blood samples different results were obtained using either blood culture technique or PCR (Table 5). Cp values of positive samples were found in the range of 32-40. PhHV signals were comparable for all samples in one run ( $\mathrm{CP} \pm 0.5)$, indicating that none of the samples contained inhibiting factors.

\section{Discussion}

In this study, we developed and evaluated the potential clinical value of a real-time PCR assay for detection of commonly encountered Gram-positive cocci from clinical samples.

The analytical sensitivity of this assay has shown to be 0.4 CFU/PCR for S. aureus, and 4 CFU/PCR for E. faecalis. Some clinical specimens (i.e. blood) contain many cells containing genomic DNA. This highly abundant genomic DNA might decrease the sensitivity of a PCR specifically detecting bacterial DNA [21]. An optimal sample processing method should diminish human DNA, concentrate pathogen DNA, and wash out inhibitory factors present in clinical specimens. As specimen preparation is of crucial importance when a sensitive test result needs to be obtained, the analytical sensitivity of the developed real-time PCR was investigated from S. aureus spiked EDTA whole blood. By using MolYsis complete 
Table 5. Comparison of blood culture results with tuf real-time PCR from blood of suspected sepsis patients.

\begin{tabular}{|c|c|c|c|}
\hline Number & Blood culture result & TTP (days) & PCR result \\
\hline 1 & Staphylococcus epidermidis & 1 & CoNS \\
\hline 2 & Not done & & CoNS \\
\hline 3 & Negative & & Negative \\
\hline 4 & Negative & & Negative \\
\hline 5 & Staphylococcus aureus & 6 & S. aureus \\
\hline 6 & Negative & & Negative \\
\hline 7 & Enterococcus faecium & $<1$ & CoNS \\
\hline 8 & Negative & & Negative \\
\hline 9 & Negative & & Negative \\
\hline 10 & Negative & & Negative \\
\hline 11 & Negative & & Negative \\
\hline 12 & Negative & & Negative \\
\hline 13 & Not done & & Negative \\
\hline 14 & Negative & & Negative \\
\hline 15 & Klebsiella pneumoniae & 1 & CoNS \\
\hline 16 & Not done & & Negative \\
\hline 17 & Not done & & Negative \\
\hline 18 & Not done & & Negative \\
\hline 19 & Bacillus fragilis & 1 & Negative \\
\hline 20 & Negative & & Negative \\
\hline 21 & Negative & & Negative \\
\hline 22 & Negative & & CoNS \\
\hline 23 & Not done & & Negative \\
\hline 24 & Negative & & Negative \\
\hline 25 & Not done & & Negative \\
\hline 26 & Staphylococcus aureus & $<1$ & S. aureus \\
\hline 27 & Negative & & Negative \\
\hline 28 & Negative & & Negative \\
\hline 29 & Negative & & CoNS \\
\hline 30 & Not done & & CoNS \\
\hline
\end{tabular}

CoNS: coagulase-negative staphylococci; TTP: time to positivity 
(Molzym) a detection limit of $0.5 \mathrm{CFU} / \mathrm{PCR}$ was obtained, which is similar as the detection limit obtained from spiked Tris-buffer.

The S. aureus and Enterococcus spp. probe are shown to be $100 \%$ specific. The crosshybridisation observed of the Staphylococcus spp. probe with L. monocytogenes is a limitation of this assay. However, as this assay is proposed to be used in combination with Gram staining, the observed aspecific PCR detection of Listeria is considered less important due to the fact that this pathogen shows a different Gram stain. Additionally, Listeria is a food-borne pathogen and is not commonly described to cause bloodstream infections as staphylococci and enterococci do [3, 22]. A potential limitation of this assay, as previously discussed by Sakai et al. [10], is that diseases resulting from mixed infections of both $S$. aureus and CoNS will not be correctly identified by this assay. If both probes show a positive signal, one would automatically conclude the presence of $S$. aureus bacteria and not the presence of CoNS. This also holds true for mixed infections of CoNS with Enterococcus spp.

Several clinical applications of the tuf gene based PCR assay were investigated in which the detection of $S$. aureus, CoNS, and enterococci are of importance; 1) blood cultures containing Gram-positive cocci, and 2) whole blood from patients of the ICU.

Firstly, the multiplex PCRs described were evaluated using 317 blood culture bottles of the BacT/ALERT system. All positive cultures containing Gram-positive cocci were detected with $100 \%$ sensitivity and specificity. In addition, all double infections were correctly identified with this assay. CoNS were detected in blood culture negative bottles (16.6\%) as well as in positive bottles containing Gram negatives (13.4\%). Both Kocuglu et al. [23] and Shigei et al. [24] describe a 3-6\% false negative rate in culture negative blood culture bottles. These false negative bottles mostly result, when subcultured, in Gram positives (83.3\%) [23]. However, no DNA detection was used to investigate false negative rates in those studies, indicating that more false negatives might have been detected when PCR based methods were used due to the fact that this is a more sensitive method. When taking into account the Cp levels obtained in the present study, CoNS contamination might be indicated in cases where the Cp value is higher than 39 and true false negatives in culture when the $\mathrm{Cp}$ value is lower than 39. This needs to be further investigated in more detail using a larger sample group to draw definitive conclusions. Although the PCR described is potentially of use for determination of S. aureus, CoNS, and Enterococcus spp. from positive blood cultures, the new technology matrix assisted laser desorption ionisation-time of flight mass spectrometry (MALDI-TOF MS) is an even faster alternative for pathogen identification from positive cultures. This relatively new technique has shown its value in the microbiology laboratory for identification of bacterial strains from positive blood cultures. However, not every diagnostic laboratory can afford a MALDI-TOF MS system. Due to the high sensitivity of this tuf based PCR assay it can be applied directly on positive blood culture bottles, but, also on blood cultures with reduced incubation times [25]. MALDI-TOF MS can thus only be applied on positive blood cultures, because $>10^{7}$ CFU needs to be used for this technique [26]. 
In addition to blood cultures, the application of the assay for whole blood samples was studied. When using the MolYsis Complete kit on EDTA whole blood samples of patients suspected of having a bloodstream infection, a similar result between blood culture results and PCR data was found in $81.8 \%$ of the samples. For one patient it took 6 days for the culture bottle to become positive for $S$. aureus, whereas PCR results were available within 4 hours (including pathogen DNA isolation). It cannot be excluded that CoNS are causative agents of disease as this group of microorganisms is increasingly detected in nosocomial infections [27-29]. Bacterial loads in these samples can be low and antibiotics could be administered to patients before blood cultures were taken resulting in negative culture results and positive PCR signals. Although strict regulations and precautions are part of standard procedures in our hospital, contamination from skin during blood sample taking cannot be excluded. Double infections can also explain differences between culture and PCR data. We are able to detect double infections in a sensitive way in reconstruction experiments (data not shown) and in blood cultures. However, in this whole blood pilot study only single infections were detected by PCR as well as in blood cultures. A positive blood culture for K. pneumoniae could not be confirmed by this PCR (Table 1); however PCR showed the presence of CoNS, which might indicate a double infection in this patient. Commercially available sepsis tests for whole blood, for example SepsiTest (Molzym), SeptiFAST (Roche) and MagicPlex Sepsis Test (Seegene), are currently under investigation in several laboratories. Preliminary results obtained with these assays [30-33] showed that real-time multiplex PCR has potential value in addition to blood culture. This is in agreement with the outcome in this small pilot study. However, molecular testing still needs further optimization and detection of more clinical relevant pathogens besides $S$. aureus, CoNS, and Enterococcus spp. needs to be added in blood based diagnostics. Furthermore, a more extended clinical evaluation, using a larger group of patients, is of the essence.

In summary, sensitive and specific tuf gene based real-time multiplex PCR assays were developed for $S$. aureus, CoNS, and Enterococcus spp., which enabled fast and accurate detection from positive blood cultures. In a small pilot study using whole blood samples of ICU patients suspected of having bloodstream infection, the developed PCR assays combined with a sensitive bacterial DNA isolation method have shown potential use in detecting frequently encountered Gram-positive cocci. These results justify further clinical evaluation of this PCR assay in a larger group of patients.

\section{Acknowledgements}

We thank Jitske Stalpers, Mariëlle Bierma, Christel van Herk, Jan Michielse, Paul van Kaathoven and Gitta Stienen for their technical support. 


\section{References}

1. Alberti, C., et al., Epidemiology of sepsis and infection in ICU patients from an international multicentre cohort study. Intensive Care Med, 2002. 28(2): p. 108-21.

2. Bearman, G.M. and R.P. Wenzel, Bacteremias: a leading cause of death. Arch Med Res, 2005. 36(6): p. 646-59.

3. von Eiff, C., et al., Infections associated with medical devices: pathogenesis, management and prophylaxis. Drugs, 2005. 65(2): p. 179-214.

4. Kumar, A., et al., Duration of hypotension before initiation of effective antimicrobial therapy is the critical determinant of survival in human septic shock. Crit Care Med, 2006. 34(6): p. 158996.

5. Annane, D., E. Bellissant, and J.M. Cavaillon, Septic shock. Lancet, 2005. 365(9453): p. 6378.

6. Klaschik, S., et al., Real-time PCR for detection and differentiation of gram-positive and gramnegative bacteria. J Clin Microbiol, 2002. 40(11): p. 4304-7.

7. Kilic, A., et al., Triplex real-time polymerase chain reaction assay for simultaneous detection of Staphylococcus aureus and coagulase-negative staphylococci and determination of methicillin resistance directly from positive blood culture bottles. Diagn Microbiol Infect Dis, 2010. 66(4): p. 349-55.

8. Riyaz-Ul-Hassan, S., V. Verma, and G.N. Qazi, Evaluation of three different molecular markers for the detection of Staphylococcus aureus by polymerase chain reaction. Food Microbiol, 2008. 25(3): p. 452-9.

9. Klouche, M. and U. Schroder, Rapid methods for diagnosis of bloodstream infections. Clin Chem Lab Med, 2008. 46(7): p. 888-908.

10. Sakai, H., et al., Simultaneous detection of Staphylococcus aureus and coagulase-negative staphylococci in positive blood cultures by real-time PCR with two fluorescence resonance energy transfer probe sets. J Clin Microbiol, 2004. 42(12): p. 5739-44.

11. Casalta, J.P., et al., Evaluation of the LightCycler(R) SeptiFast test in the rapid etiologic diagnostic of infectious endocarditis. Eur J Clin Microbiol Infect Dis, 2009. 28(6): p. 569-73.

12. Horz, H.P., et al., Selective isolation of bacterial DNA from human clinical specimens. J Microbiol Methods, 2008. 72(1): p. 98-102.

13. Rantakokko-Jalava, K. and J. Jalava, Optimal DNA isolation method for detection of bacteria in clinical specimens by broad-range PCR. J Clin Microbiol, 2002. 40(11): p. 4211-7.

14. Smith, K., M.A. Diggle, and S.C. Clarke, Comparison of commercial DNA extraction kits for extraction of bacterial genomic DNA from whole-blood samples. J Clin Microbiol, 2003. 41(6): p. 2440-3.

15. Hansen, W.L., C.A. Bruggeman, and P.F. Wolffs, Evaluation of new preanalysis sample treatment tools and DNA isolation protocols to improve bacterial pathogen detection in whole blood. J Clin Microbiol, 2009. 47(8): p. 2629-31.

16. Loens, K., et al., Evaluation of NucliSens easyMAG for automated nucleic acid extraction from various clinical specimens. J Clin Microbiol, 2007. 45(2): p. 421-5.

17. Heikens, E., et al., Comparison of genotypic and phenotypic methods for species-level identification of clinical isolates of coagulase-negative staphylococci. J Clin Microbiol, 2005. 43(5): p. 2286-90.

18. Martineau, F., et al., Development of a PCR assay for identification of staphylococci at genus and species levels. J Clin Microbiol, 2001. 39(7): p. 2541-7.

19. Bone, R.C., et al., Definitions for sepsis and organ failure and guidelines for the use of innovative therapies in sepsis. The ACCP/SCCM Consensus Conference Committee. American College of Chest Physicians/Society of Critical Care Medicine. Chest, 1992. 101(6): p. 1644-55.

20. van Doornum, G.J., et al., Diagnosing herpesvirus infections by real-time amplification and rapid culture. J Clin Microbiol, 2003. 41(2): p. 576-80.

21. Sachse, S., et al., Truncated human cytidylate-phosphate-deoxyguanylate-binding protein for improved nucleic acid amplification technique-based detection of bacterial species in human samples. J Clin Microbiol, 2009. 47(4): p. 1050-7. 
22. Neely, A.N. and M.P. Maley, Survival of enterococci and staphylococci on hospital fabrics and plastic. J Clin Microbiol, 2000. 38(2): p. 724-6.

23. Kocoglu, M.E., A. Bayram, and I. Balci, Evaluation of negative results of BacT/Alert 3D automated blood culture system. J Microbiol, 2005. 43(3): p. 257-9.

24. Shigei, J.T., et al., Value of terminal subcultures for blood cultures monitored by BACTEC 9240. J Clin Microbiol, 1995. 33(5): p. 1385-8.

25. Loonen, A.J., et al., Acceleration of the direct identification of Staphylococcus aureus versus coagulase-negative staphylococci from blood culture material: a comparison of six bacterial DNA extraction methods. Eur J Clin Microbiol Infect Dis, 2011. 30(3): p. 337-42.

26. Christner, M., et al., Rapid identification of bacteria from positive blood culture bottles by use of matrix-assisted laser desorption-ionization time of flight mass spectrometry fingerprinting. J Clin Microbiol, 2010. 48(5): p. 1584-91.

27. Kloos, W.E. and T.L. Bannerman, Update on clinical significance of coagulase-negative staphylococci. Clin Microbiol Rev, 1994. 7(1): p. 117-40.

28. Piette, A. and G. Verschraegen, Role of coagulase-negative staphylococci in human disease. Vet Microbiol, 2009. 134(1-2): p. 45-54.

29. Stefani, S. and P.E. Varaldo, Epidemiology of methicillin-resistant staphylococci in Europe. Clin Microbiol Infect, 2003. 9(12): p. 1179-86.

30. Lehmann, L.E., et al., A multiplex real-time PCR assay for rapid detection and differentiation of 25 bacterial and fungal pathogens from whole blood samples. Med Microbiol Immunol, 2008. 197(3): p. 313-24.

31. Tsalik, E.L., et al., Multiplex PCR to diagnose bloodstream infections in patients admitted from the emergency department with sepsis. J Clin Microbiol, 2010. 48(1): p. 26-33.

32. Wallet, F., et al., Preliminary clinical study using a multiplex real-time PCR test for the detection of bacterial and fungal DNA directly in blood. Clin Microbiol Infect, 2010. 16(6): p. 774-779.

33. Wellinghausen, N., et al., Diagnosis of bacteremia in whole-blood samples by use of a commercial universal 16S rRNA gene-based PCR and sequence analysis. J Clin Microbiol, 2009. 47(9): p. 2759-65. 


\section{Chapter 3}

Acceleration of direct identification of Staphylococcus aureus

versus coagulase-negative staphylococci from blood culture

material: a comparison of six bacterial DNA extraction methods

Anne J.M. Loonen ${ }^{1,2,3}$, Arjan R. Jansz', Herman Kreeftenberg ${ }^{4}$, Cathrien A. Bruggeman ${ }^{3}$, Petra F.G. Wolffs ${ }^{3}$, and Adriaan J.C. van den Brule ${ }^{1,2,5}$

1 PAMM Laboratory for Medical Microbiology, Eindhoven/Veldhoven, The Netherlands ${ }_{2}^{2}$ Fontys University of Applied Science, Centre for Medical Molecular Diagnostics, Eindhoven, The Netherlands ${ }^{3}$ Maastricht University Medical Centre, CAPHRI, Department of Medical Microbiology, Maastricht, The Netherlands ${ }^{4}$ Catharina Hospital, Department of Intensive Care, Eindhoven, The Netherlands

${ }^{5}$ Catharina Hospital, Department of Molecular Diagnostics, Eindhoven, The Netherlands

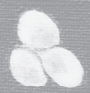

Eur J Clin Microbiol Infect Dis 2011, Mar;30(3):337-42 


\section{Abstract}

To accelerate differentiation between Staphylococcus aureus and coagulase-negative staphylococci (CoNS), this study aimed to compare six different DNA extraction methods from two commonly used blood culture materials, i.e. BACTEC and BacT/ALERT. Furthermore, we analysed the effect of reduced blood culture incubation for detection of staphylococci directly from blood culture material.

A real-time polymerase chain reaction (PCR) duplex assay was used to compare the six different DNA isolation protocols on two different blood culture systems. Negative blood culture material was spiked with methicillin-resistant $S$. aureus (MRSA). Bacterial DNA was isolated with automated extractor EasyMAG (three protocols), automated extractor MagNA Pure LC (LC Microbiology Kit MGrade), a manual kit MolYsis Plus and a combination of MolYsis Plus and the EasyMAG. The most optimal isolation method was used to evaluate reduced bacterial incubation times.

Bacterial DNA isolation with the MolYsis Plus kit in combination with the specific B protocol on the EasyMAG resulted in the most sensitive detection of $S$. aureus, with a detection limit of $10 \mathrm{CFU} / \mathrm{ml}$, in BacT/ALERT material, whereas using BACTEC resulted in a detection limit of $100 \mathrm{CFU} / \mathrm{ml}$.

An initial S. aureus or CoNS load of $1 \mathrm{CFU} / \mathrm{ml}$ blood can be detected after $5 \mathrm{~h}$ of incubation in BacT/ALERT 3D by combining the sensitive isolation method and the tuf LightCycler assay. 


\section{Introduction}

Staphylococcus aureus is a pathogen which can cause both hospital- and community associated infectious diseases, ranging from minor skin infections to endocarditis, bacteremia, sepsis and septic shock [1]. Sepsis can result in high morbidity and mortality. In the United States, bloodstream infections develop in approximately 250.000 people annually [2]. In The Netherlands, the incidence of patients admitted to the intensive care unit (ICU) with severe sepsis is in the range of around $8643 \pm 929$ per year [3]. Currently, blood culture is the gold standard for identification of pathogens from suspected bacterial sepsis patients. Unfortunately, blood culture is time-consuming, taking at least 24-72 hours for the final determination of the bacteria causing the disease. Staphylococci are the most common Gram-positive organisms in blood cultures. Differentiating S. aureus from coagulasenegative staphylococci (CoNS) is important, because sepsis with $S$. aureus is common and virulent with mortality rates in the range of $20-30 \%$ [4]. CoNS are often considered as being contaminants in blood cultures due to the fact that these species are members of the normal skin flora and mucous membranes, and can contaminate the sample when it is taken. However, it is known that CoNS infections are increasingly recognized as clinically relevant infections and confirmation on the presence of these species in blood culture is, therefore, important (reviewed in [5-8]). Several molecular methods for rapid and accurate detection of bacteria from positive blood culture material have been described, including (commercial) real-time PCR based diagnostic tests [9-11], fluorescence in situ hybridization $[12,13]$, MALDI-TOF MS [14], and also DNA micro-arrays [15, 16]. However, all of these techniques are used on positive blood culture material.

Reduction in the time to obtaining results can be achieved by applying molecular methods either directly on whole blood or on blood culture material with reduced incubation times. Ideally, usage of whole blood is preferred but the techniques that are now available are often not sensitive enough, clinically, as has been shown by others investigating a commercial real-time PCR test currently available [17-19]. Blood culture materials are known to contain inhibiting factors which can reduce detection in a sensitive real-time PCR [20-23]. It is, therefore, important to include a good isolation method in the molecular diagnostic strategy, which is able to efficiently remove inhibiting factors and one which still allows sensitive DNA detection by PCR.

In this study we compared six different, both manual and automated, bacterial DNA isolation methods for two commonly used blood culture systems, i.e. BACTEC (Becton Dickinson) and BacT/ALERT (bioMérieux), to be able to find the most sensitive bacterial DNA isolation method. Additionally, we investigated the occurrence of inhibition in PCR amplification after DNA isolation. A sensitive real-time PCR assay was designed to be able to detect staphylococci and to differentiate S. aureus from CoNS [Loonen et al., manuscript submitted]. 
Subsequently, this real-time PCR was used in combination with the optimal DNA isolation method to investigate the level of time reduction to identify staphylococci from blood culture material. The results were compared with conventional blood culture techniques used in diagnostic laboratories.

\section{Materials and Methods}

\section{Culture methods}

Negatively cultured blood culture bottles, derived from routine diagnostics of both BACTEC (Becton Dickinson, The Netherlands) and BacT/ALERT (bioMérieux, The Netherlands) systems, were used in this study for spiking experiments (bottles have been cultured for 1 week and remained negative). Additionally, healthy volunteers donated $10 \mathrm{ml}$ blood as the input for the BacT/ALERT bottles. The bottles were incubated and handled according to manufacturer's protocol.

\section{DNA isolation methods}

Methicillin-resistant S. aureus (MRSA) ATCC 33592 was cultured overnight on blood agar. Fresh plates were used to make a 2-McF solution which was subsequently serially diluted (1:10) in negative blood culture material from both systems (BACTEC and BacT/ALERT). One hundred $\mu \mathrm{l}$ was plated on blood agar plates to check the number of CFU per sample. Three different isolation protocols on the NucliSens EasyMAG (bioMérieux) were tested (generic, specific A and specific $B$ protocol: the main difference between protocols is the number of washing steps, with least steps in the generic protocol and most washing steps in the specific B protocol), one isolation kit (LC Microbiology Kit $\mathrm{M}^{\text {Grade }}$ ) on the MagNA Pure LC (Roche Diagnostics), the MolYsis Plus kit (Molzym GmbH, Bremen, Germany) was used according to Molzym's instructions and in combination with the EasyMAG. When MolYsis was used in combination with the EasyMAG, manufacturer's instructions (Molzym) were followed until the Buglysis step and, subsequently, bacterial DNA was extracted according to the specific $B$ protocol on the EasyMAG. Input volume in each isolation was $200 \mu l$, only for the MagNA Pure LC $100 \mu \mathrm{l}$ was used, and samples were always eluted in $100 \mu$ l elution buffer provided with each isolation method. Each extraction was performed at least 2-3 times in independent experiments. Phocine herpes virus type 1 (PhHV-1) was added to each sample eluate as an amplification and inhibition control. Samples were analysed with the LightCycler assay described below.

\section{LightCycler assay}

A duplex real-time PCR, detecting the tuf gene of both staphylococci and $S$. aureus specifically, is used as described in detail elsewhere [Loonen et al., submitted]. In brief, the 
positions of the primers and probes were derived from the $S$. aureus tuf gene sequence (GenBank accession no. AF298796). The sequences of the primers and probes were as follows: Staphylococcus spp. forward primer ccaatgccacaaactcgtga (position 32-51), Staphylococcus spp. reverse primer cacgaccagtgattgagaatacg (position 371-349), S. aureus forward primer tcctggttcaattacaccacatactg (position 583-608), S. aureus reverse primer ggaaatagaattgtggacgatagtttga (position 698-671), Staphylococcus spp. probe VICccattcatgatgccagttg-BHQ1 (position 326-344), S. aureus probe FAM- tgataatacgtatacttctgcBHQ1 (position 637-617). Oligonucleotide primers were obtained from Eurogentec (Liège, Belgium), Staphylococcus XS probe and the $S$. aureus XS probe were obtained from Biolegio (Nijmegen, The Netherlands). PhHV-1 primers and VIC-labeled probe [24] were obtained from Applied Biosystems (Nieuwerkerk aan den IJssel, The Netherlands). PCR mixtures consisted of 5x LightCycler TaqMan Master mixture (Roche Diagnostics), $0.9 \mu \mathrm{M}$ concentration of each primer, $0.2 \mu \mathrm{M}$ concentration of each probe, and aquadest in a volume of $15 \mu \mathrm{l}$ to which $5 \mu \mathrm{l}$ DNA sample was added, leading to a final reaction volume of $20 \mu \mathrm{l}$ in each capillary. Primers and probe targeting PhHV had a concentration of $0.1 \mu \mathrm{M}$ each and were used in a separate single real-time PCR. All samples were amplified on the LightCycler v2.0 (Roche Diagnostics) according to the following cycling parameters: an initial incubation step for $10 \mathrm{~min}$ at $95^{\circ} \mathrm{C}$ for enzyme activation and DNA denaturation, then, 45 cycles of $15 \mathrm{~s}$ $95^{\circ} \mathrm{C}$ followed by $1 \mathrm{~min} 60^{\circ} \mathrm{C}$. The results were analysed with LightCycler Software version 4.05 (Roche Diagnostics).

\section{Results}

\section{Evaluation of different bacterial DNA extraction methods}

In Table 1, the results are presented of the comparison of six different bacterial DNA extraction methods on $S$. aureus spiked blood culture material. The MagNA Pure LC (Roche Diagnostics) resulted in a detection limit of $10^{4} \mathrm{CFU} / \mathrm{ml}$ for both culture systems. On the EasyMAG, three protocols were evaluated to isolate bacterial DNA from spiked clinical material, generic, specific $A$ and specific $B$. We tested all three protocols and usage of the specific $B$ protocol resulted in the best detection limit of $10^{2} \mathrm{CFU} / \mathrm{ml}$ of $\mathrm{S}$. aureus in BacT/ ALERT material. Although MolYsis Plus did not result in a low detection limit, we tested this manual kit in combination with the specific B protocol on the EasyMAG. By combining both methods, all human DNA was removed and bacteria were lysed by the MolYsis Plus kit, and, subsequently, the EasyMAG was used for optimal washing and elution of bacterial DNA. It was possible to reduce hands-on time and avoid possible contamination due to skipping of manual handlings. This combination of methods resulted in the most sensitive detection limit, i.e.10 CFU/ml, in BacT/ALERT material. The time to obtaining results for this combined method followed by real-time PCR is approximately $4-5 \mathrm{~h}$, but it strongly depends on the 
Table 1. Evaluation of six different DNA isolation protocols on blood culture material spiked with tenfold dilution series of Staphylococcus aureus

\begin{tabular}{lllllll}
\hline & \multicolumn{7}{c}{ Detection limit in CFU/mI } \\
DNA isolation protocol & $10^{5}$ & $10^{4}$ & $10^{3}$ & $10^{2}$ & 10 & 1 \\
\hline EasyMAG generic & $+^{*}$ & $+^{*}$ & + & - & - & - \\
EasyMAG specific A & $+^{*}$ & $+^{*}$ & $+^{*}$ & - & - & - \\
EasyMAG specific B & $+^{*}$ & $+^{*}$ & $+^{*}$ & + & - & - \\
MagNA Pure LC & $+^{*}$ & $+^{*}$ & - & - & - & - \\
MolYsis Plus & $+^{*}$ & $+^{*}$ & - & - & - & - \\
MolYsis Plus + EasyMAG specific B & $+^{*}$ & $+^{*}$ & $+^{*}$ & $+^{*}$ & + & - \\
\hline
\end{tabular}

*BACTEC; + BacT/ALERT; - not detected

number of samples. No inhibition was observed in the real-time PCR, and all samples and controls showed similar $\mathrm{Cp}$ values in one run $(\mathrm{Cp} \pm 0.5)$. All experiments were performed at least 2-3 times.

\section{Reduced blood culture times}

To investigate the effect of reduced blood culture incubation for pathogen detection by PCR, a combination between the sensitive tuf LightCycler assay and the sensitive isolation method, the MolYsis Plus Kit in combination with the specific B protocol on the EasyMAG, was analysed on S. aureus (ATCC 33592)-spiked BacT/ALERT blood culture bottles containing fresh blood of healthy volunteers. First, it was investigated how many CFU of $S$. aureus per $\mathrm{ml}$ needed to be present to be able to detect a signal with the LightCycler assay, therefore, $1.000,100$ and 10 CFU were spiked in different bottles, both SA (Standard Aerobic) and SN (Standard Anaerobic) BacT/ALERT bottles. BACTEC bottles were not investigated due to poorer results obtained with all isolation methods analysed. The results of this experiment are depicted in Figure 1 and indicate that $1.000 \mathrm{CFU}$ of $S$. aureus $(100 \mathrm{CFU} / \mathrm{ml}$ blood) could be detected by PCR immediately after spiking, indicating that no incubation step was required. The bottles containing $100 \mathrm{CFU}$ of $\mathrm{S}$. aureus (10 CFU/ml blood) resulted in positive real-time PCR signals after $1 \mathrm{~h}$ of incubation in the BacT/ALERT 3D. The 10-CFU bottles (1 $\mathrm{CFU} / \mathrm{ml}$ blood) needed a maximum of $5 \mathrm{~h}$ of incubation in the BacT/ALERT3D to be able to detect the $S$. aureus bacteria present using a subsequent PCR. The experiment with $10 \mathrm{CFU}$ $S$. aureus per bottle was repeated eight times with both aerobe and anaerobe bottles and showed reproducible results. Standard deviations (SD) of the $\mathrm{Cp}$ values were calculated for the $10 \mathrm{CFU} /$ bottle experiments (aureus probe). The 3-h time point (2/8 positive) showed an SD of 3.44, the 4-h time point (5/8 positive) indicated an SD of 1.09 , and the 5-h time point (8/8 positive) resulted in an SD of 1.09. Clinical strains of $S$. aureus and $S$. epidermidis were also spiked in BacT/ALERT blood culture bottles (aerobe (SA) and anaerobe (SN)) containing 
A

\begin{tabular}{lllllll}
\hline & \multicolumn{7}{c}{ Timepoints (hours) } \\
\hline Bacterial load CFU/bottle & $\mathbf{0}$ & $\mathbf{1}$ & $\mathbf{2}$ & $\mathbf{3}$ & $\mathbf{4}$ & $\mathbf{5}$ \\
1.000 & $1 / 1$ & $1 / 1$ & $1 / 1$ & $1 / 1$ & $1 / 1$ & $1 / 1$ \\
100 & $0 / 1$ & $1 / 1$ & $1 / 1$ & $1 / 1$ & $1 / 1$ & $1 / 1$ \\
10 & $0 / 8$ & $0 / 8$ & $0 / 8$ & $2 / 8$ & $5 / 8$ & $8 / 8$ \\
\hline
\end{tabular}

B

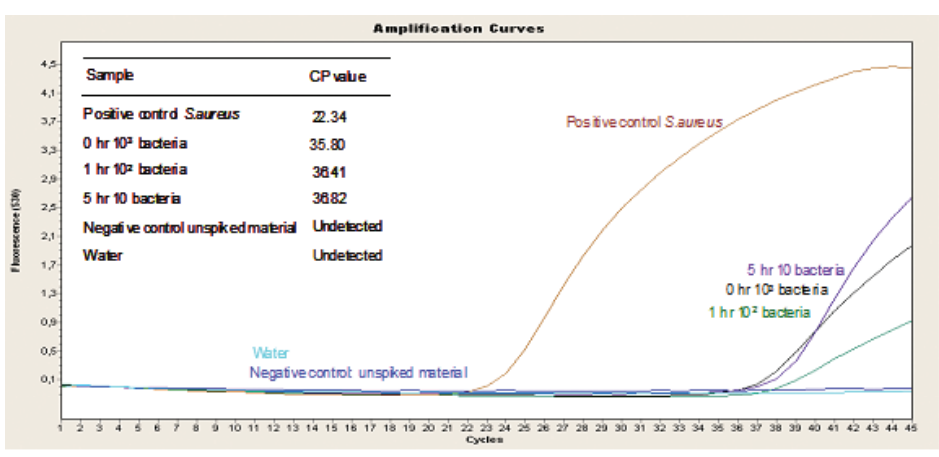

Figure 1. Staphylococcus aureus detection in relation to reduced bacterial culture times. A, Overview of the detection of $S$. aureus in relation to reduced culture times. BacT/ALERT blood culture bottles with blood of healthy volunteers were spiked with $S$. aureus and samples were taken every hour. The experiments with 1.000 and 100 bacteria per bottle were performed once. The 1.000 bacteria/bottle did not need a pre-culture step, a tuf real-time PCR signal could be detected immediately after spiking $(0 \mathrm{hr})$. The 100 bacteria/bottle needed a pre-culture step of 1 hour in the BacT/ALERT 3D to obtain a positive signal in the real-time PCR. The experiment with 10 bacteria/ bottle (10 ml blood) was repeated eight times. Real-time PCR signal could be detected after a preculture step of 3-5 hours. All bottles, 8 of 8 , were positive after $5 \mathrm{~h}$ of pre-culture. Standard deviations (SD) for 10 CFU/bottle were calculated as follows: $3 \mathrm{hr}$ SD 3.44, $4 \mathrm{hr}$ SD 1.09, and $5 \mathrm{hr}$ SD 1.09. B, LightCycler tuf real-time PCR result in the $530 \mathrm{~nm}$ fluorescence channel (S. aureus probe).

the blood of healthy volunteers. The results of these experiments were similar as the results presented for the $S$. aureus ATCC 33592 strain. With conventional detection methods, the results of the bottles containing $10 \mathrm{CFU}$ of $S$. aureus were available after at least $28 \mathrm{~h}$.

\section{Discussion}

In this study, six different bacterial DNA extraction protocols were evaluated, by using a sensitive and specific tuf gene based real-time PCR detection assay, which enabled the differentiation between $S$. aureus and CoNS. From this study, it can be concluded that the 
MolYsis Plus kit (Molzym) in combination with the specific B protocol on the EasyMAG (bioMérieux) resulted in the most optimal isolation of bacterial DNA from BacT/ALERT blood culture material. A detection limit of $10 \mathrm{CFU} / \mathrm{ml}$ was obtained in the tuf LightCycler assay for BacT/ALERT material. By using the tuf LightCycler assay, $1 \mathrm{CFU} / \mathrm{ml}$ of $\mathrm{S}$. aureus or CoNS could be detected from BacT/ALERT blood culture material when an incubation step of a maximum of $5 \mathrm{~h}$ was applied. By using this reduced incubation time, it was possible to identify $S$. aureus versus CoNS within one working day.

Six different bacterial DNA extraction methods were compared on two commonly used blood culture systems (BACTEC and BacT/ALERT). The specific B protocol on the EasyMAG contains the most washing steps compared to the generic and specific A protocol, possibly explaining the better results obtained with this isolation protocol. The bacterial DNA isolation performed with the MolYsis Plus kit resulted in a low detection limit for both culture systems. Gebert et al., published a study in 2008 in which they show that the MolYsis Plus kit performs well in their hands [25]. They used $500 \mu$ input material and were able to conclude that PCR analysis in combination with MolYsis Plus DNA isolation resulted in accelerated detection of pathogens in blood culture samples. Inhibiting factors were not found to be present in the samples in the study of Gebert et al. [25]. In our study, no PCR inhibition was observed (all samples and controls showed similar $\mathrm{Cp} \pm 0,5$ values in one run). The detection limit in the LightCycler assay, after MolYsis Plus isolation, was low, i.e. $10^{4} \mathrm{CFU} / \mathrm{ml}$. The MolYsis Plus kit eliminates human background DNA from the samples and enrichment of bacterial DNA takes place. Complete isolation with the MolYsis Plus kit takes $3 \mathrm{~h}$, of which $2 \mathrm{~h}$ is hands-on time. By using the EasyMAG specific B protocol after completion of the MolYsis Plus Buglysis step, the total isolation time is not significantly reduced. However, hands-on time and the chance for contamination are drastically reduced, due to the fact that column handling is skipped. A comparison between both culture systems resulted in a difference in bacterial DNA isolation efficiency. The use of BacT/ALERT blood culture material lead, in most isolation methods, to better results compared to BACTEC material. We experienced difficulties in the bacterial DNA isolations due to the beads present in BACTEC bottles, but we cannot conclude that the beads lead to the weaker performance of BACTEC material compared to BacT/ALERT material. However, due to this finding, all experiments were continued with BacT/ALERT material.

By applying a 5-h incubation step for blood culture bottles containing approximately 10 CFU of $S$. aureus or CoNS, it was possible to detect these bacteria by the tuf LightCycler assay. These results could be produced within one working day when starting early in the morning. Compared to conventional culture techniques for staphylococci, the results were available at least $19 \mathrm{~h}$ faster. Gebert et al. [25], also showed in their paper that, by combining a sensitive real-time PCR and a sensitive isolation method, culture times can be reduced. They showed a 5.0-8.7 $\mathrm{h}$ time gain in spiking experiments and an average time gain of 10.7 
h was obtained when the method was applied on blood culture bottles of septic patients. The maximum time gain can be reached when starting either immediately in the morning or when the microbiology laboratory has $24-\mathrm{h}$ service. Laboratories with a $24-\mathrm{h}$ setup will always benefit from this method.

The experiments described in this paper all focus on accelerated detection of staphylococci, especially on detection of $S$. aureus. Staphylococci were chosen as a model system because Gram-positive bacteria are frequently detected in blood culture bottles, making it a relevant group of microorganisms for such a study [26-28]. Infections are, however, caused by a variety of pathogens. Staphylococci are fast-growing bacteria and, in this study, a proof-of-principle is shown. The accelerated detection, of other pathogens causing bloodstream infections (i.e. Escherichia coli), directly from blood culture material in combination with reduced culture time and with this sensitive isolation method should be investigated in future experiments. It is of clinical importance in the future to focus especially on fastidious pathogens or fungi, since those pathogens are difficult to culture and, for these pathogens, time to result can then, hopefully, be drastically reduced. A similar approach as presented in this study can be applied to investigate reduced incubation times for other pathogens.

It is known from literature that staphylococci are the most frequently causative agent of medical-device-related and surgical-site infections [7]. Therefore, it is interesting to apply the tuf LightCycler duplex assay in these patient groups using whole blood samples. The preliminary results showed that the MolYsis Complete kit (Molzym) has already proven to be successful in our hands (data not shown), as well as in the hands of others [29].

In conclusion, by combining the MolYsis Plus kit and the specific B protocol on the EasyMAG, $10 \mathrm{CFU} / \mathrm{ml}$ could be detected from $\mathrm{S}$. aureus spiked BacT/ALERT blood culture material. By applying this isolation method followed by the sensitive tuf LightCycler duplex assay, using 5-h-incubated spiked blood culture material, 1 CFU S. aureus or CoNS per $1 \mathrm{ml}$ blood could be detected. Future studies are needed in order to address the clinical application of this approach.

\section{Acknowledgements}

We thank Wendy Hansen, Jitske Stalpers, Mariëlle Bierma, Christel van Herk, Paul van Kaathoven, Gitta Stienen and Jan Michielse for their technical support. 


\section{References}

1. Lowy, F.D., Staphylococcus aureus infections. N Engl J Med, 1998. 339(8): p. 520-32.

2. CDC, Report of mortality statistics. Monthly Vital Statistics Report, 1992. 40(11): p. 1-23.

3. van Gestel, A., et al., Prevalence and incidence of severe sepsis in Dutch intensive care units. Crit Care, 2004. 8(4): p. R153-62.

4. Saginur, R. and K.N. Suh, Staphylococcus aureus bacteraemia of unknown primary source: where do we stand? Int J Antimicrob Agents, 2008. 32 Suppl 1: p. S21-5.

5. von Eiff, C., G. Peters, and C. Heilmann, Pathogenesis of infections due to coagulasenegative staphylococci. Lancet Infect Dis, 2002. 2(11): p. 677-85.

6. Piette, A. and G. Verschraegen, Role of coagulase-negative staphylococci in human disease. Vet Microbiol, 2009. 134(1-2): p. 45-54.

7. Huebner, J. and D.A. Goldmann, Coagulase-negative staphylococci: role as pathogens. Annu Rev Med, 1999. 50: p. 223-36.

8. Kloos, W.E. and T.L. Bannerman, Update on clinical significance of coagulase-negative staphylococci. Clin Microbiol Rev, 1994. 7(1): p. 117-40.

9. Wellinghausen, N., et al., Algorithm for the identification of bacterial pathogens in positive blood cultures by real-time LightCycler polymerase chain reaction (PCR) with sequencespecific probes. Diagn Microbiol Infect Dis, 2004. 48(4): p. 229-41.

10. Sakai, H., et al., Simultaneous detection of Staphylococcus aureus and coagulase-negative staphylococci in positive blood cultures by real-time PCR with two fluorescence resonance energy transfer probe sets. J Clin Microbiol, 2004. 42(12): p. 5739-44.

11. Granger, K., et al., Multiplex PCR-Ligation Detection Reaction assay for the simultaneous detection of drug resistance and toxin genes from Staphylococcus aureus, Enterococcus faecalis and Enterococcus faecium. J Clin Microbiol, 2009. 48: p. 277-280.

12. Hartmann, H., et al., Rapid identification of Staphylococcus aureus in blood cultures by a combination of fluorescence in situ hybridization using peptide nucleic acid probes and flow cytometry. J Clin Microbiol, 2005. 43(9): p. 4855-7.

13. Peters, R.P., et al., Rapid identification of pathogens in blood cultures with a modified fluorescence in situ hybridization assay. J Clin Microbiol, 2006. 44(11): p. 4186-8.

14. Stevenson, L.G., S.K. Drake, and P.R. Murray, Rapid Identification of Bacteria in Positive Blood Culture Broths by MALDI-TOF Mass Spectrometry. J Clin Microbiol, 2010. 48(2): p. 4447.

15. Yoo, S.M., et al., DNA microarray-based identification of bacterial and fungal pathogens in bloodstream infections. Mol Cell Probes, 2010. 24: p. 44-52.

16. Cleven, B.E., et al., Identification and characterization of bacterial pathogens causing bloodstream infections by DNA microarray. J Clin Microbiol, 2006. 44(7): p. 2389-97.

17. Dierkes, C., et al., Clinical impact of a commercially available multiplex PCR system for rapid detection of pathogens in patients with presumed sepsis. BMC Infect Dis, 2009. 9: p. 126.

18. Wallet, F., et al., Preliminary clinical study using a multiplex real-time PCR test for the detection of bacterial and fungal DNA directly in blood. Clin Microbiol Infect, 2010. 16(6): p. 774-779.

19. Avolio, M., et al., Molecular identification of bloodstream pathogens in patients presenting to the emergency department with suspected sepsis. Shock, 2010. 34(1): p. 27-30.

20. Wilson, I.G., Inhibition and facilitation of nucleic acid amplification. Appl Environ Microbiol, 1997. 63(10): p. 3741-51.

21. Al-Soud, W.A. and P. Radstrom, Purification and characterization of PCR-inhibitory components in blood cells. J Clin Microbiol, 2001. 39(2): p. 485-93.

22. Millar, B.C., et al., A simple and sensitive method to extract bacterial, yeast and fungal DNA from blood culture material. J Microbiol Methods, 2000. 42(2): p. 139-47.

23. Fredricks, D.N. and D.A. Relman, Improved amplification of microbial DNA from blood cultures by removal of the PCR inhibitor sodium polyanetholesulfonate. J Clin Microbiol, 1998. 36(10): p. 2810-6. 
24. van Doornum, G.J., et al., Diagnosing herpesvirus infections by real-time amplification and rapid culture. J Clin Microbiol, 2003. 41(2): p. 576-80.

25. Gebert, S., D. Siegel, and N. Wellinghausen, Rapid detection of pathogens in blood culture bottles by real-time PCR in conjunction with the pre-analytic tool MolYsis. J Infect, 2008. 57: p. 307-316.

26. Alberti, C., et al., Epidemiology of sepsis and infection in ICU patients from an international multicentre cohort study. Intensive Care Med, 2002. 28(2): p. 108-21.

27. Annane, D., E. Bellissant, and J.M. Cavaillon, Septic shock. Lancet, 2005. 365(9453): p. 6378.

28. Martin, G.S., et al., The epidemiology of sepsis in the United States from 1979 through 2000. N Engl J Med, 2003. 348(16): p. 1546-54.

29. Hansen, W.L., C.A. Bruggeman, and P.F. Wolffs, Evaluation of new preanalysis sample treatment tools and DNA isolation protocols to improve bacterial pathogen detection in whole blood. J Clin Microbiol, 2009. 47(8): p. 2629-31. 



\section{Chapter 4}

Comparative study using phenotypic, genotypic and proteomics methods for identification of coagulase-negative staphylococci

PAMM Laboratories, Department of Medical Microbiology, Eindhoven/Veldhoven, The Netherlands ${ }^{2}$ Fontys University of Applied Science, Department of Medical Molecular Diagnostics, Eindhoven, The Netherlands

${ }_{3}^{3}$ Maastricht University Medical Centre, CAPHRI, Department of Medical Microbiology, Maastricht, The Netherlands

${ }^{4}$ Jeroen Bosch Hospital, laboratory for Molecular Diagnostics, Department of Medical Microbiology and Pathology,

's-Hertogenbosch, The Netherlands

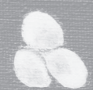

J Clin Microbiology 2012, Apr;50(4):1437-9 


\begin{abstract}
Five methods were compared to determine the most accurate method for identification of coagulase-negative staphylococci (CoNS) ( $n=142$ strains). Matrix-assisted laser desorption ionization time-of-flight mass spectrometry (MALDI-TOF MS) showed the best results for rapid and accurate CoNS differentiation (99.3\% of strains correctly identified). An alternative to this approach could be VITEK 2 combined with partial tuf gene sequencing ( $100 \%$ of strains correctly identified when both methods are performed simultaneously).
\end{abstract}


Coagulase-negative staphylococci (CoNS) have emerged as significant pathogens and are mainly found in hospitalized immunocompromised patients, often with indwelling or implanted medical devices [1-5]. Most infections are hospital acquired, and CoNS infections can result in several diseases, including bloodstream infection, endocarditis, mediastinitis, meningitis, urinary tract infections (Staphylococcus saprophyticus), and medical-devicerelated infections [1, 2, 6-9]. Accurate identification of CoNS is important when CoNS are isolated from multiple blood cultures of 1 patient. Many CoNS are described to be resistant to multiple antibiotics [7, 10-12]. Matrix-assisted laser desorption ionization time-of-flight mass spectrometry (MALDI-TOF MS) is a relatively new technique that allows examination of protein profiles from bacteria, and this technique has been shown to work in a fast and accurate manner [13-16].

In the present comparative study, five methods for differentiation of CoNS were compared, i.e., VITEK 2 (Gram Positive card REF 21342, bioMérieux), the ID 32 Staph strip (bioMérieux), partial 16S rRNA gene sequencing (MicroSeq, Applied Biosystems), partial tuf gene sequencing (inhouse), and MALDI-TOF MS (Bruker Daltonics) on the same set of CoNS strains in order to find the most suitable method available today for identification of staphylococci.

A total of 142 CoNS strains were included in this study. Isolates were derived from clinical cultures ( $n=117)$, and 25 reference strains from the American Type Culture Collection (ATCC) and the National Institute for Health and Environment (RIVM, Bilthoven, The Netherlands) were included. The clinical isolates were selected from positive blood cultures (two or more positive cultures from 1 patient), urinary tract infections (S. saprophyticus), or deep-seated infections. The CoNS species name that was found with $\geq 3$ of the 5 used methods was chosen as the true CoNS species name (referred to here as the true ID). When only 2 methods resulted in a similar true IDs (4/142 cases [2.8\%]), tuf sequencing was selected as most reliable method, as the result for 16S rRNA gene sequencing was noninformative (Staphylococcus spp.).

CoNS strains were cultured overnight at $35^{\circ} \mathrm{C}$ on blood plates (made in-house). The phenotypical methods VITEK 2 (bioMérieux, Marcy L'Etoile, France) and ID 32 Staph (bioMérieux, Marcy L'Etoile, France) were both performed as described by the manufacturer. DNA for sequencing was isolated from 2 McFarland bacterial suspensions by heating them (for $10 \mathrm{~min}$ at $95^{\circ} \mathrm{C}$ ). The $16 \mathrm{~S}$ rRNA primers from the MicroSeq kit were used, and these primers amplify approximately the first $500 \mathrm{bp}$ of the gene (Applied Biosystems, Foster City, CA). The tuf forward primer (tuf_32_FW) was described previously [17], and the tuf reverse primer (tuf_512_RV: 5'-AGCTTCAGCGTAGTCTAATAATTTACG-'3) was designed for this study. Positions of the tuf primers were derived from the Staphylococcus aureus tuf gene sequence (GenBank accession no. AF298796), and these primers amplify a 480bp fragment. The tuf primers were obtained from Eurogentec (Liège, Belgium). PCR was performed according to standard procedures. Sequencing was performed using the Big Dye 
XTerminator sequencing mix 3.1 (Applied Biosystems, Foster City, CA) and ran on an $\mathrm{ABI}$ 310 Prism genetic analyzer (Applied Biosystems, Foster City, CA). The 16S DNA sequences were analysed by using MicroSeqID Analysis software (Applied Biosystems, Foster City, CA). The tuf DNA sequences were analysed by using the Basic Local Assignment Search Tool (BLAST, http://blast.ncbi.nlm.nih.gov/Blast.cgi).

For MALDI-TOF MS (Bruker Daltonics) analysis, 1 colony of each CoNS strain was spotted with a sterile wooden stick, in duplicate, on the polished steel target plate. Samples that could not be identified directly by MALDI-TOF MS were retested after pre-treatment $(n=5$ clinical CoNS strains [1 strain each of Staphylococcus hominis, S. epidermidis, S. cohnii, S. schleiferi, and S. saprophyticus]) as described by van Veen et al., [13]. However, in this study formic acid $(70 \%)$ and acetonitrile were added according to pellet size at a $1: 1$ ratio. The BioTyper database version V3 1.1.0_3476-3740 was used as reference.

The results (Table 1) of the phenotypic identification methods used in this study indicate that the VITEK 2 performs best, with a correct-identification rate of $92.3 \%$, compared to $85.9 \%$ for ID 32 Staph. The acceptance criteria for all results are described in the footnote of Table 1. With the use of $16 \mathrm{~S}$ rRNA and tuf sequencing, $70.4 \%$ and $93 \%$ of the CoNS strains, respectively, were correctly identified. When the first hit with the MicroSeqID database was selected as true finding (disregarding analysis rules), then 137 out of 142 strains were correctly identified (96.5\%). Application of the CLSI guidelines [18], which are stricter than those used in this study, will lead to even more results that are noninformative (Staphylococcus spp.) and thus incorrect identifications with $16 \mathrm{~S}$ sequencing in this study. Whole-16S-gene sequencing will improve the Staphylococcus spp. differentiation ability of this specific gene but will also increase the number of required sequence reactions and consequently slightly increase costs and, in some cases, time-to results. Becker et al., [19] showed with the use of the quality-controlled Ribosomal Sequence Database (RIDOM), identification of staphylococci improved from $83.6 \%$ to $98.2 \%$ compared to the results obtained with the NCBI database. With the use of the RIDOM database, the results obtained in this study might positively change as well. However, tuf gene sequencing resulted in more informative data (species name) and was also in concordance with MALDI-TOF MS results. Although all Staphylococcus warneri strains $(n=10)$ resulted in incorrect identification with tuf gene sequencing, due to high homology to Staphylococcus pasteuri, extensive analysis of S. pasteuri and S. warneri strains might solve the current problems. Therefore, the tuf gene seems more suitable for identification of staphylococci, as described by others [20, 21].

The MALDI-TOF MS data obtained in this evaluation indicate the superiority in identification of CoNS, as $99.3 \%$ of the strains were correctly identified with this method. Strains were spotted in duplicate and resulted in the same identification (strain name). The database (V3 1.1.0_3476-3740) used for this study contained the following numbers of reference spectra per tested CoNS species: Staphylococcus capitis (6), S. caprae (1), S. cohnii (5), 
Table 1. Summary of the performance of methods for differentiation of CoNS*

\begin{tabular}{|c|c|c|c|c|c|c|c|c|c|c|c|}
\hline \multirow[b]{3}{*}{ True organism } & \multirow[b]{3}{*}{ No. of strains } & \multicolumn{10}{|c|}{ No. of strains identified by indicated method } \\
\hline & & \multicolumn{2}{|c|}{ ID 32 Staph } & \multicolumn{2}{|c|}{ Vitek2 } & \multicolumn{2}{|c|}{$16 \mathrm{~S}$ sequencing } & \multicolumn{2}{|c|}{ tuf sequencing } & \multicolumn{2}{|c|}{ MALDI-TOF MS } \\
\hline & & Correctly & Incorrectly & Correctly & Incorrectly & Correctly & Incorrectly & Correctly & Incorrectly & Correctly & Incorrectly \\
\hline S.capitis & 13 & 12 & 1 & 12 & 1 & 11 & 2 & 13 & & 13 & \\
\hline S.caprae & 1 & & 1 & 1 & & & 1 & 1 & & 1 & \\
\hline S.cohnii & 1 & 1 & & 1 & & & 1 & 1 & & 1 & \\
\hline S.epidermidis & 29 & 24 & 5 & 26 & 3 & 24 & 5 & 29 & & 29 & \\
\hline S. haemolyticus & 18 & 18 & & 16 & 2 & 13 & 5 & 18 & & 18 & \\
\hline S.hominis & 22 & 19 & 3 & 21 & 1 & 17 & 5 & 22 & & 21 & 1 \\
\hline S.lentus & 1 & 1 & & 1 & & & 1 & 1 & & 1 & \\
\hline S.lugdunensis & 11 & 11 & & 11 & & 9 & 2 & 11 & & 11 & \\
\hline S.pasteuri & 1 & & 1 & & 1 & 1 & & 1 & & 1 & \\
\hline S.pettenkoferi & 2 & & 2 & & 2 & & 2 & 2 & & 2 & \\
\hline S.saprophyticus & 12 & 12 & & 12 & & 8 & 4 & 12 & & 12 & \\
\hline S.schleiferi & 10 & 4 & 6 & 10 & & 4 & 6 & 10 & & 10 & \\
\hline S.sciuri & 5 & 5 & & 5 & & & 5 & 5 & & 5 & \\
\hline S. simulans & 6 & 6 & & 5 & 1 & 6 & & 6 & & 6 & \\
\hline S. warneri & 10 & 9 & 1 & 10 & & 7 & 3 & & 10 & 10 & \\
\hline Total (\%) & $142(100)$ & $122(85.9)$ & $20(14.1)$ & $131(92.3)$ & $11(7.7)$ & $100(70.4)$ & $42(29.6)$ & $132(93)$ & $10(7)$ & 141 (99.3) & $1(0.7)$ \\
\hline
\end{tabular}

*The acceptance criteria for the results were as follows: for ID 32 Staph, a score of $\geq 70 \%$; for VITEK 2 a score of $\geq 70 \%$ (in-house validation); for tuf sequencing, a species name provided by BLAST showing ${ }^{3} 99 \%$ sequence match (identity) and a mismatch of at least 1 nucleotide with the second hit; for $16 \mathrm{~S}$ sequencing, a first hit showing ${ }^{3} 99 \%$ sequence match (identity) and a second hit having a mismatch of at least 1 nucleotide (MicroSeq software); for MALDI-TOF MS, a score of $\geq 1.7$, however, $<2$ were considered reliable to the species level if the first and second database matches resulted in the same strain name; ideally, $\geq 2$ reference spectra need to be available in the database, and scores $\geq 2$ were considered reliable to the species level.

S. epidermidis (9), S. haemolyticus (8), S. hominis (6), S. lentus (2), S. lugdunensis (5), S. pasteuri (2), S. pettenkoferi (4), S. saprophyticus (8), S. schleiferi (6), S. sciuri (4), S. simulans (5), and S. warneri (4). For S. caprae, only 1 reference spectrum is available, and duplicate measurement indicated $S$. caprae (hit 1: score, 1.853). The second hit for this CoNS species indicated $S$. epidermidis twice (<1.5). For the proposed result acceptance criteria for MALDITOF MS to be used, the number of reference spectra per CoNS strain ideally needs to be $\geq 2$. However, as hit 2 provided an unreliable score for $S$. epidermidis, we considered the $S$. caprea result as a correct identification. On average, there was a $4.82 \%$ difference in score value between hit 1 and 2 . The combination of MALDI-TOF MS with tuf gene sequencing resulted in a correct-identification rate of $100 \%$.

The 25 reference strains used in this study showed that different misidentifications occurred with the investigated methods. With the $16 \mathrm{~S}$ sequencing protocol 9/25 reference strains could not be identified to the species level (36\%). For the VITEK 2, ID32 Staph, and tuf sequencing 2/25 reference strains were misidentified (8\%). MALDI-TOF MS resulted in correct identification of all reference strains. MALDI-TOF MS has been investigated by others in relation to Staphylococcus species identification directly from agar plates [15, 16, 22, 23], and the correct-identification rates range from $74.2 \%-99.3 \%$. The data obtained in this study 
are comparable with data reported by Spanu et al., [23] and Dubois et al. [16], in which a sensitivity of $99.3 \%$ was found. In this study, only one $S$. hominis strain could not be identified with MALDI-TOF MS (Table 1), for unknown reasons.

Sequencing of the partial $16 \mathrm{~S}$ gene resulted in most clinical relevant misidentifications, as both S. Iugdunensis and S. saprophyticus were not correctly identified.

In conclusion, this is one of the most extensive comparative studies for CoNS identification. The results obtained in this study demonstrate the good performance of MALDI-TOF MS for identification of CoNS, a correct identification rate of $99.3 \%$ was achieved with this method. tuf gene sequencing is the most suitable substitute for MALDI-TOF MS. When cheap, fast and accurate identification of CoNS is needed, the usage of MALDI-TOF MS is the method of choice, and when necessary, the tuf gene can be sequenced. As an alternative method, due to the fact that not every diagnostic microbiology laboratory has access to a MALDI-TOF MS system, VITEK 2 combined with tuf sequencing is suggested as an accurate approach for CoNS differentiation.

\section{Acknowledgements}

We thank Jitske Stalpers and Christel van Herk for their technical support. 


\section{References}

1. Pfaller, M.A. and L.A. Herwaldt, Laboratory, clinical, and epidemiological aspects of coagulase-negative staphylococci. Clin Microbiol Rev, 1988. 1(3): p. 281-99.

2. von Eiff, C., et al., Infections associated with medical devices: pathogenesis, management and prophylaxis. Drugs, 2005. 65(2): p. 179-214.

3. Kloos, W.E. and T.L. Bannerman, Update on clinical significance of coagulase-negative staphylococci. Clin Microbiol Rev, 1994. 7(1): p. 117-40.

4. Hira, V., et al., Clinical and molecular epidemiologic characteristics of coagulase-negative staphylococcal bloodstream infections in intensive care neonates. Pediatr Infect Dis J, 2007. 26(7): p. 607-12.

5. Krediet, T.G., et al., Molecular epidemiology of coagulase-negative staphylococci causing sepsis in a neonatal intensive care unit over an 11-year period. J Clin Microbiol, 2004. 42(3): p. 992-5.

6. Karchmer, A.W., Nosocomial bloodstream infections: organisms, risk factors, and implications. Clin Infect Dis, 2000. 31 Suppl 4: p. S139-43.

7. Piette, A. and G. Verschraegen, Role of coagulase-negative staphylococci in human disease. Vet Microbiol, 2009. 134(1-2): p. 45-54.

8. Garcia, P., et al., Coagulase-negative staphylococci: clinical, microbiological and molecular features to predict true bacteraemia. J Med Microbiol, 2004. 53(Pt 1): p. 67-72.

9. Bearman, G.M. and R.P. Wenzel, Bacteremias: a leading cause of death. Arch Med Res, 2005. 36(6): p. 646-59.

10. Huebner, J. and D.A. Goldmann, Coagulase-negative staphylococci: role as pathogens. Annu Rev Med, 1999. 50: p. 223-36.

11. Natoli, S., et al., Characterization of coagulase-negative staphylococcal isolates from blood with reduced susceptibility to glycopeptides and therapeutic options. BMC Infect Dis, 2009. 9: p. 83.

12. Koksal, F., H. Yasar, and M. Samasti, Antibiotic resistance patterns of coagulase-negative staphylococcus strains isolated from blood cultures of septicemic patients in Turkey. Microbiol Res, 2007.

13. van Veen, S.Q., E.C. Claas, and E.J. Kuijper, High-throughput identification of bacteria and yeast by matrix-assisted laser desorption ionization-time of flight mass spectrometry in conventional medical microbiology laboratories. J Clin Microbiol, 2010. 48(3): p. 900-7.

14. Carbonnelle, E., et al., Rapid identification of Staphylococci isolated in clinical microbiology laboratories by matrix-assisted laser desorption ionization-time of flight mass spectrometry. J Clin Microbiol, 2007. 45(7): p. 2156-61.

15. Dupont, C., et al., Identification of clinical coagulase-negative staphylococci, isolated in microbiology laboratories, by matrix-assisted laser desorption/ionization-time of flight mass spectrometry and two automated systems. Clin Microbiol Infect, 2010. 16: p. 998-1001.

16. Dubois, D., et al., Identification of a variety of Staphylococcus species by MALDI-TOF mass spectrometry. J Clin Microbiol, 2010. 48: p. 941-945.

17. Loonen, A.J., et al., Acceleration of the direct identification of Staphylococcus aureus versus coagulase-negative staphylococci from blood culture material: a comparison of six bacterial DNA extraction methods. Eur J Clin Microbiol Infect Dis, 2011. 30(3): p. 337-42.

18. Wayne, P.A., Interpretive criteria for identification of bacteria and fungi by DNA target sequencing; approved guidline. CLSI document MM18-A. Clinical Laboratory Standards Institute, 2008: p. 26-30.

19. Becker, K., et al., Development and evaluation of a quality-controlled ribosomal sequence database for $16 S$ ribosomal DNA-based identification of Staphylococcus species. J Clin Microbiol, 2004. 42(11): p. 4988-95.

20. Heikens, E., et al., Comparison of genotypic and phenotypic methods for species-level identification of clinical isolates of coagulase-negative staphylococci. J Clin Microbiol, 2005. 43(5): p. 2286-90. 
21. Carpaij, N., et al., Comparison of the identification of coagulase-negative staphylococci by matrix-assisted laser desorption ionization time-of-flight mass spectrometry and tuf sequencing. Eur J Clin Microbiol Infect Dis, 2011. 30(10): p. 1169-72.

22. Bergeron, M., et al., Species identification of staphylococci by amplification and sequencing of the tuf gene compared to the gap gene and by matrix-assisted laser desorption ionization time-of-flight mass spectrometry. Eur J Clin Microbiol Infect Dis, 2011. 30(3): p. 343-54.

23. Spanu, T., et al., Evaluation of matrix-assisted laser desorption ionization-time-of-flight mass spectrometry in comparison to rpoB gene sequencing for species identification of bloodstream infection staphylococcal isolates. Clin Microbiol Infect, 2011. 17: p. 44-49. 


\title{
Chapter 5
}

\section{Speciation of coagulase-negative staphylococci from positive}

\author{
blood cultures using MALDI-TOF MS: clinical relevance
}

Judith Beuving ${ }^{1,2}$, Anne J.M. Loonen ${ }^{1,3,4}$, Margreet C. Vos ${ }^{5}$ Adriaan J.C. van den Brule ${ }^{3,4}$, Annelies Verbon ${ }^{1,6}$, and Petra F.G. Wolffs ${ }^{1}$

${ }_{1}$ Maastricht University Medical Centre, Department of Medical Microbiology, Maastricht, The Netherlands

2 St Elisabeth Hospital, Department of Medical Microbiology, Tilburg, The Netherlands

3 Jeroen Bosch Hospital, Laboratory for Molecular Diagnostics, Departments of Medical Microbiology and

Pathology, 's Hertogenbosch, The Netherlands

${ }^{4}$ Fontys University of Applied Sciences, Department of Medical Molecular Diagnostics, Eindhoven, The Netherlands

${ }^{5}$ Erasmus Medical Centre, Department of Medical Microbiology and Infectious Diseases, ${ }^{6}$ Department of Internal Medicine, division of infectious diseases, Rotterdam, The Netherlands

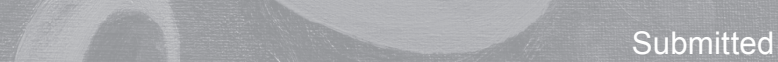




\section{Abstract}

The incidence of coagulase-negative staphylococci (CoNS) is rising, however, CoNS are also the most common blood culture contaminants. MALDI-TOF MS offers a cheap and reliable method for identification to species level of CoNS. In this study, we investigated the clinical relevance of various CoNS species isolated from positive blood cultures in a two center retrospective cohort study.

In two Dutch hospitals, CoNS blood culture isolates were collected and identified to species level using MALDI-TOF MS. Whether an isolate represented a bloodstream infection or contamination was established by CDC-criteria and by medical microbiologists or infectious diseases specialists.

265 strains were collected from 245 patients. Staphylococcus epidermidis was the most common species found $(n=182,68.7 \%)$ and the majority of these strains represented contamination (83.5\% (CDC criteria) and 59.3\% (clinical microbiologists/infectious diseases specialists)). It was also the most common cause of CoNS bloodstream infections $(66.7 \%$ (CDC criteria) and $75.5 \%$ (clinical microbiologists/infectious diseases specialists)). In comparison to the other CoNS species, Staphylococcus haemolyticus was significantly more frequently considered to cause a bloodstream infection (40.0\% (CDC criteria) and $70.0 \%$ (clinical microbiologists/infectious diseases specialists)). Some species, such as Staphylococcus warneri, were found to be always contaminants when using the CDC criteria. Clinicians were less likely to regard a strain as contaminant compared to the diagnosis according to the CDC-criteria.

In conclusion, determining whether or not a positive blood culture with CoNS represents a bloodstream infection needs standard use of criteria such as those of the CDC. This study shows that the various CoNS species vary considerably in pathogenicity. Speciation of CoNS by MALDI-TOF MS can therefore be helpful in establishing whether a patient is suffering from an actual CoNS bloodstream infection. 


\section{Introduction}

Coagulase-negative staphylococci (CoNS) have long been considered to be blood culture contaminants in the majority of patients. However, nowadays, CoNS is considered to be a true pathogen in special patient groups or conditions [1,2]. Several species can cause infections without the presence of foreign body material, such as $S$. lugdunensis and $S$. schleiferi causing bloodstream infections or endocarditis, and S. saprophyticus causing urinary tract infections [3]. However, most CoNS bacteremias are associated with prosthetic device and intravenous catheter infections [4].

Whether a CoNS from a grown blood culture represents true bacteremia or a contaminant is usually based on the number of grown blood culture vials and clinical data, for which strict CDC-criteria are available (www.cdc.gov/nhsnpdfs/pscmanual/4psc_clabscurrent. pdf). However, in clinical practice, treatment for CoNS bacteremia is also frequently started or maintained in patients not meeting these criteria [5]. Until now, it has been unclear whether identification of subspecies of CoNS may be helpful in the discrimination between contamination and infection.

Phenotypic methods (VITEK 2, API system) for identification of CoNS to species level are unreliable [6, 7]. PCR followed by sequencing is more reliable but also time-consuming and expensive. Furthermore, the gene most commonly used for identification of bacteria, the 16S rRNA-gene, is not specific enough for differentiation of different species of CoNS [6]. Therefore, up until now, most laboratories used coagulase production and DNAse production to discern CoNS from S. aureus and do not further determine the species of the CoNS strain, except in selected cases.

The recent introduction of MALDI-TOF MS systems, however, offers a cheap, rapid and reliable method for identification of staphylococci $[6,8,9]$. Therefore, identification to the species level of Staphylococcus strains can more easily be performed in routine patient care. It is necessary to evaluate the value of this further identification in the diagnosis of infection versus contamination. Therefore, we investigated the clinical relevance of various CoNS species isolated from positive blood cultures in a two center retrospective cohort study and compared the outcome to identification of the Staphylococcal species.

\section{Materials and Methods}

\section{Ethics statement}

All data and strains in this study were collected retrospectively and anonimised before analysis. No consent from the patients was required and the ethics committee did not have to be approached. This is in agreement with the code for proper use of human tissue as formulated by the Dutch Federation of Medical Scientific Societies. 


\section{Sample collection}

Strains were collected in two hospitals: the Erasmus MC, Medical Centre in Rotterdam, The Netherlands, a 1200-bed university hospital, and the Maastricht University Medical Centre, Maastricht, The Netherlands, a 750-bed university hospital.

In Rotterdam, blood culture CoNS strains were collected retrospectively between January and June 2011. In Maastricht, blood culture samples were collected between October 2009 and May 2011. Only one blood culture was included per patient. If this blood culture yielded 2 strains of CoNS, both strains were included in the study. Exclusion criteria were: insufficient clinical data, age $<18$ years, and blood cultures with mixed cultures containing non-CoNS strains.

\section{Blood cultures}

In both centres, blood cultures bottles were cultured and automatically monitored for microbial growth in the BACTEC ${ }^{\text {TM }} 9240$ instrument (BD Diagnostic Systems, Sparks, MD, USA). If growth was detected, blood culture broth was subcultured on Columbia sheep blood agar. From this subculture, Staphylococcus species were tested for clumping factor and DNAse production. If both tests were negative, the strain was identified as coagulase-negative Staphylococcus. In case of discrepancy between both tests, a tube coagulase test was performed as a gold standard.

In both centres, coagulase-negative staphylococci were not routinely identified to species level.

Antibiotic susceptibility testing was performed by automated systems: VITEK 2 system (bioMérieux, Marcy L'Etoile, France) in Rotterdam and BD Phoenix system (BD Diagnostic Systems, Sparks, MD, USA) in Maastricht. All strains were stored at $-80^{\circ} \mathrm{C}$.

\section{MALDI-TOF MS}

Strains stored at $-80^{\circ} \mathrm{C}$ were subcultured twice on Columbia sheep blood agar, after which MALDI-TOF MS analysis was performed in duplicate, as was described elsewhere [6]. Scores $<1.7$ were considered to be unreliable, scores between 1.7 and 2 were considered to be reliable to the genus level; however, if database match number 1 and 2 resulted in the same strain name, it was considered correct to the species level, and scores $\geq 2$ were considered to be reliable to the species level. Samples that could not be identified directly by MALDI-TOF MS were retested after pretreatment with formic acid and acetonitrile [6].

\section{CDC-criteria for laboratory-confirmed bloodstream infection}

A patient with a positive blood culture with CoNS was considered to have a bloodstream infection if the following criteria were met: fever $\left(>38^{\circ} \mathrm{C}\right)$ or chills or hypotension, and: positive laboratory results are not related to an infection at another site, and CoNS cultured 
from two or more blood cultures drawn on separate occasions (www.cdc.gov/nhsnpdfs/ pscmanual/4psc_clabscurrent.pdf).

\section{Data collection}

The following clinical data were collected from the patients' electronic medical record: number of blood cultures drawn within 24 hours of drawing of the positive blood culture, number of positive blood cultures, antibiotic susceptibility of all blood cultures growing CoNS, positive cultures from other body sites, presence or absence of fever $\left(>38^{\circ} \mathrm{C}\right)$ within 24 hours of blood culture drawing, presence or absence of hypotension (systolic blood pressure $<90 \mathrm{mmHg}$ ), other potential foci of fever, results of consultations of clinical microbiologists and infectious diseases specialists regarding the positive blood culture.

\section{Statistical analysis}

For comparison of the percentage of strains causing infections between the various species, Chi-square tests were used. Differences between groups with a $p$-value $<0.05$ were considered to be statistically significant.

\section{Results}

\section{Identification of CoNS strains}

In total, 304 strains were collected, 172 in a six months period in Rotterdam and 132 in a fifteen months period in Maastricht. These strains were cultured from 279 blood cultures. Four patients were excluded because their strains failed to grow after storage at $-80^{\circ} \mathrm{C}$. Of 29 patients, insufficient clinical data were available. One blood culture was excluded because it grew $>1$ genus (CoNS and Escherichia coli). This resulted in 265 strains (152 from Rotterdam and 113 from Maastricht) belonging to 245 patients being included in the study for analysis (Figure 1).

From 18 cultures, more than one species of CoNS was cultured, based on colony morphology and/or antibiotic resistance pattern: 17 grew 2 species and 1 grew 4 species.

The most commonly isolated species was $S$. epidermidis, followed by $S$. hominis, $S$. capitis and S. haemolyticus. S. hominis was almost twice as common in Maastricht than in Rotterdam, however, this did not reach statistical significance. Overall, no significant differences $(p=0.60)$ were observed between the two hospitals in species distribution. No $S$. lugdunensis or S. schleiferi were found. (Table 1)

Six strains were identified by MALDI-TOF MS as not being Staphylococcus species: two were Rothia mucilaginosa, four were Micrococcus luteus. One strain could not reliably be identified to species level in four attempts. 


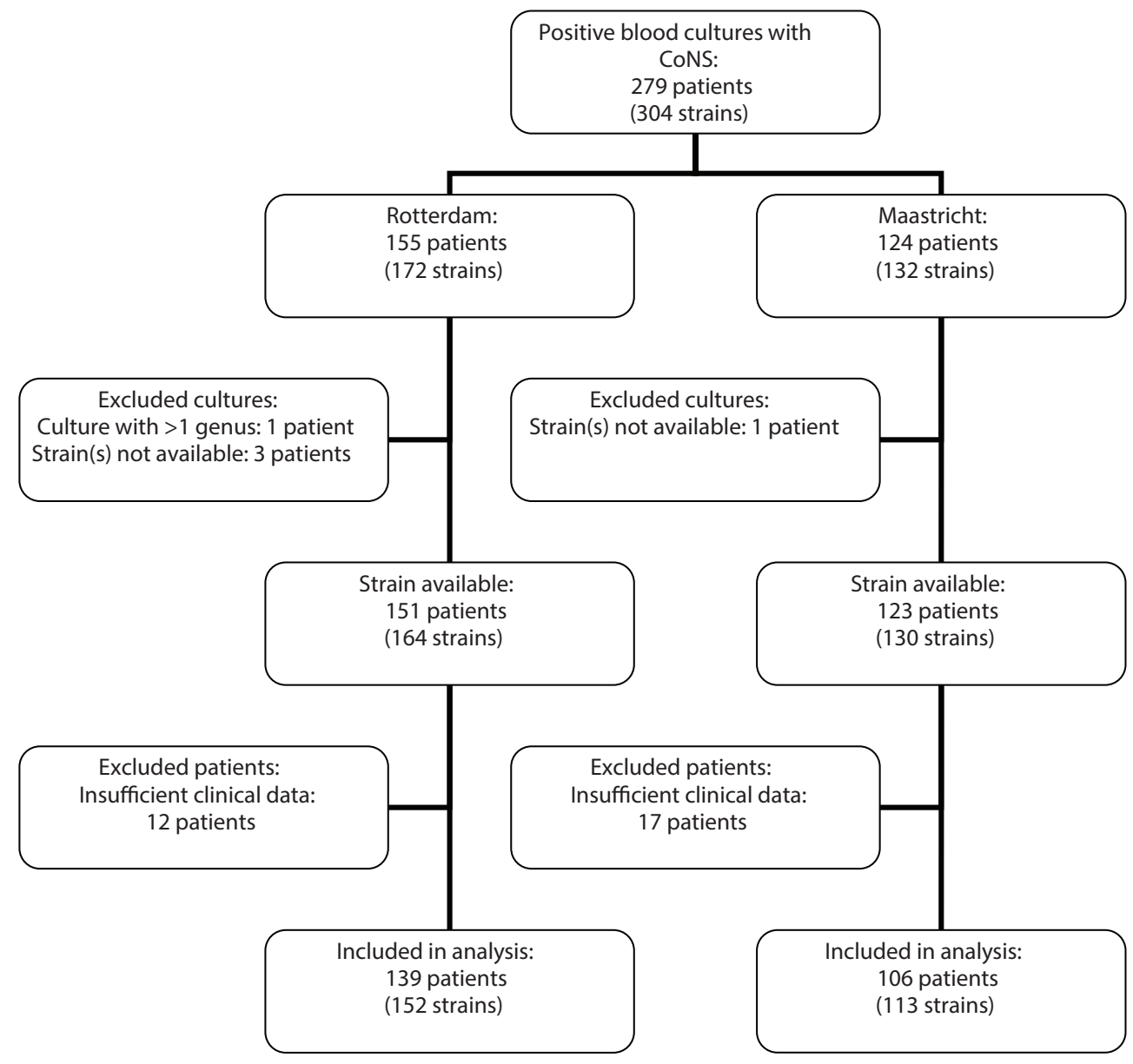

Figure 1. Flowchart showing included patients and strains.

\section{Diagnosis of infection with CoNS}

According to the CDC-criteria, $17.0 \%$ of all positive blood cultures with CoNS represented bloodstream infections. According to the consultations of clinical microbiologists and infectious diseases specialists, $37.0 \%$ of the CoNS strains caused bloodstream infections. (Table 2), which was significantly higher than the number of CoNS infections according to CDC-criteria ( $p<0.001)$. There was no significant difference ( $p=0.549$ for the CDC-criteria, $p=0.473$ for the clinicians' consultations) between the two hospitals in these numbers.

55 isolates were thought to be causative agents of a bloodstream infections by clinicians, whereas they were regarded to be blood culture contaminants by CDC-criteria (Table 2). 
Table 1. Results of MALDI-TOF MS.

\begin{tabular}{llll}
\hline & Total number of strains & $\begin{array}{l}\text { Number of strains in } \\
\text { Rotterdam }\end{array}$ & $\begin{array}{l}\text { Number of strains in } \\
\text { Maastricht }\end{array}$ \\
\hline S. epidermidis & $182(68.7 \%)$ & $112(73.7 \%)$ & $70(61.9 \%)$ \\
S. hominis & $38(14.3 \%)$ & $16(10.5 \%)$ & $22(19.5 \%)$ \\
S. haemolyticus & $10(3.8 \%)$ & $5(3.3 \%)$ & $5(4.4 \%)$ \\
S. capitis & $17(6.4 \%)$ & $9(5.9 \%)$ & $8(7.1 \%)$ \\
S. pettenkoferi & $2(0.8 \%)$ & $1(0.7 \%)$ & $1(0.9 \%)$ \\
S. caprae & $1(0.4 \%)$ & $1(0.7 \%)$ & $0(0.0 \%)$ \\
S. saprophyticus & $1(0.4 \%)$ & $1(0.7 \%)$ & $0(0.0 \%)$ \\
Rothia mucilaginosa & $2(0.8 \%)$ & $1(0.7 \%)$ & $1(0.9 \%)$ \\
Micrococcus luteus & $4(1.5 \%)$ & $1(0.7 \%)$ & $3(2.7 \%)$ \\
S. intermedius & $1(0.4 \%)$ & $1(0.7 \%)$ & $0(0.0 \%)$ \\
S. cohnii & $2(0.8 \%)$ & $1(0.7 \%)$ & $1(0.9 \%)$ \\
S. warneri & $4(1.5 \%)$ & $2(1.3 \%)$ & $2(1.8 \%)$ \\
No ID & $1(0.4 \%)$ & $1(0.7 \%)$ & $0(0.0 \%)$ \\
\hline
\end{tabular}

Table 2. Number of true bloodstream infections, according to CDC-criteria and clinical microbiologist/infectious diseases specialist.

\begin{tabular}{|c|c|c|c|}
\hline & Total & Rotterdam & Maastricht \\
\hline Infection according to CDC-criteria & $45(17.0 \%)$ & $24(15.8 \%)$ & $21(18.6 \%)$ \\
\hline $\begin{array}{l}\text { Infection according to clinical microbiologist/ } \\
\text { infectious diseases specialist }\end{array}$ & $98(37.0 \%)$ & $59(38.8 \%)$ & $39(34.5 \%)$ \\
\hline $\begin{array}{l}\text { Infection according to clinical microbiologist/ } \\
\text { infectious diseases specialist but not according } \\
\text { to CDC-criteria }\end{array}$ & $55(20.8 \%)$ & $35(23.0 \%)$ & $20(17.7 \%)$ \\
\hline $\begin{array}{l}\text { Infection according to CDC-criteria but not } \\
\text { according to clinical microbiologist/infectious } \\
\text { diseases specialist }\end{array}$ & $2(0.8 \%)$ & $0(0.0 \%)$ & $2(1.8 \%)$ \\
\hline
\end{tabular}

Of 100 patients, only one set of blood cultures was drawn, these patients therefore could never meet CDC-criteria. Twenty-two of them were considered to have a bloodstream infection by clinical microbiologists and infectious diseases specialists. Sixty-seven patients only had one positive blood culture set (of at least two sets drawn). Of these, 4 patients were regarded by clinicians, to have a CoNS infection.

Of the 82 patients not having either hypotension or fever at the time of blood culture drawing, 19 were considered by clinicians to suffer from CoNS bloodstream infection.

In total, 36 patients grew a CoNS from a culture from another body site or removed foreign body material. These strains were not available for MALDI-TOF MS analysis and could therefore not be identified to species level. Of these 36 patients, 12 patients met CDC- 
criteria, whereas 32 were regarded to suffer from a CoNS bloodstream infection by clinical microbiologists and infectious diseases specialists.

Two positive blood cultures meeting the CDC-criteria for bloodstream infection were regarded to be a contaminant by clinical microbiologists/infectious diseases specialists.

\section{Value of identification of CoNS in diagnosing infection}

The species most often isolated in true bloodstream infections was $S$. epidermidis, representing $66.7 \%$ of all bloodstream infections by CDC-criteria, and $75.5 \%$ of infections according to clinical microbiologists or infectious diseases specialists. This was followed by S. hominis and S. haemolyticus. The vast majority (83.5\%) of isolated S. epidermidis strains represented blood culture contamination as defined by CDC-criteria, thereby, also being the species most commonly isolated from contaminated blood cultures. $S$. saprophyticus and $R$. mucilaginosa were always associated with infections (Table $3 \mathrm{~A}$ and $\mathrm{B}$ ).

S. haemolyticus represented only $3.8 \%$ of all isolates. However, of all S. haemolyticus isolates, $40.0 \%$ represented infection by CDC-criteria, and even $70.0 \%$ by the clinicians' criteria, which was significantly more often than other species $(p=0.012)$. Thereby, it represented $8.9 \%$ of all bloodstream infections by CDC-criteria (Table $3 A$ and $B$ ).

Of all species isolated more than once, S. pettenkoferi, S. cohnii, S. warneri and M. Iuteus were never associated with infection by CDC-criteria.

\section{Discussion}

In this study, we examined the added value of identification of CoNS from positive blood cultures to species level and showed that identification to species level can be helpful in determining whether an isolate is likely to be a true pathogen or a contaminant. S. haemolyticus was significantly more often associated with bloodstream infections than other CoNS species: $40.0 \%$ according to CDC-criteria and even $70.0 \%$ according to clinicians. Several species were always found to be contaminants: S. pettenkoferi, S. caprae, S. intermedius, and S. cohnii. Six strains were identified as not being staphylococci, but Rothia spp. or Micrococcus spp. However, it can be difficult to discern these strains from staphylococci by using only colony morphology, Gram-staining and catalase tests. MALDI-TOF MS can therefore be very helpful in their identification, especially since all Micrococcus strains represented contaminants and both Rothia strains were shown to be clinically relevant.

The two medical centres in this study represent two completely separate patient populations, since they both have different adherence populations. We found that the distribution of species was similar in both hospitals, as well as the ratio true pathogens/contaminants, both by CDC-criteria and as judged by clinical microbiologists and infectious diseases specialists. 
Table 3A Species distribution between contaminants and true bloodstream infection, A) by CDCcriteria and B) clinical microbiologist/infectious diseases specialist.

\begin{tabular}{lllll}
\hline $\begin{array}{l}\text { CDC-criteria: } \\
\text { contamination or } \\
\text { infection, per species }\end{array}$ & No. of strains & $\begin{array}{l}\text { No. of } \\
\text { infections }\end{array}$ & $\begin{array}{l}\% \text { of species } \\
\text { representing } \\
\text { infection }\end{array}$ & $\begin{array}{l}\% \text { of total } \\
\text { number of } \\
\text { infections }\end{array}$ \\
\hline S. epidermidis & 182 & 30 & $16.5 \%$ & $66.7 \%$ \\
S. hominis & 38 & 6 & $15.8 \%$ & $13.3 \%$ \\
S. haemolyticus & 10 & 4 & $40.0 \%$ & $8.9 \%$ \\
S. capitis & 17 & 2 & $11.8 \%$ & $4.4 \%$ \\
S. pettenkoferi & 2 & 0 & $0.0 \%$ & $0.0 \%$ \\
S. caprae & 1 & 0 & $0.0 \%$ & $0.0 \%$ \\
S. saprophyticus & 1 & 1 & $100.0 \%$ & $2.2 \%$ \\
Rothia mucilaginosa & 2 & 2 & $100.0 \%$ & $4.4 \%$ \\
Micrococcus luteus & 4 & 0 & $0.0 \%$ & $0.0 \%$ \\
S. intermedius & 1 & 0 & $0.0 \%$ & $0.0 \%$ \\
S. cohnii & 2 & 0 & $0.0 \%$ & $0.0 \%$ \\
S. warneri & 4 & 0 & $0.0 \%$ & $0.0 \%$ \\
No ID & 1 & 0 & $0.0 \%$ & $0.0 \%$ \\
Total & 265 & 45 & $17.0 \%$ & \\
\hline
\end{tabular}

Table 3B

\begin{tabular}{|c|c|c|c|c|}
\hline $\begin{array}{l}\text { Clinical microbiologist/ } \\
\text { infectious diseases } \\
\text { specialist: } \\
\text { contamination or infection, } \\
\text { per species }\end{array}$ & No. of strains & $\begin{array}{l}\text { No. of } \\
\text { infections }\end{array}$ & $\begin{array}{l}\% \text { of species } \\
\text { representing } \\
\text { infection }\end{array}$ & $\begin{array}{l}\% \text { of total } \\
\text { number of } \\
\text { infections }\end{array}$ \\
\hline S. epidermidis & 182 & 74 & $40.7 \%$ & $75.5 \%$ \\
\hline S. hominis & 38 & 7 & $18.4 \%$ & $7.1 \%$ \\
\hline S. haemolyticus & 10 & 7 & $70.0 \%$ & $7.1 \%$ \\
\hline S. capitis & 17 & 6 & $35.3 \%$ & $6.1 \%$ \\
\hline S. pettenkoferi & 2 & 0 & $0.0 \%$ & $0.0 \%$ \\
\hline S. caprae & 1 & 0 & $0.0 \%$ & $0.0 \%$ \\
\hline S. saprophyticus & 1 & 1 & $100.0 \%$ & $1.0 \%$ \\
\hline Rothia mucilaginosa & 2 & 2 & $100.0 \%$ & $2.0 \%$ \\
\hline Micrococcus luteus & 4 & 0 & $0.0 \%$ & $0.0 \%$ \\
\hline S.intermedius & 1 & 0 & $0.0 \%$ & $0.0 \%$ \\
\hline S. cohnii & 2 & 0 & $0.0 \%$ & $0.0 \%$ \\
\hline S. warneri & 4 & 1 & $25.0 \%$ & $1.0 \%$ \\
\hline No ID & 1 & 0 & $0.0 \%$ & $0.0 \%$ \\
\hline Total & 265 & 98 & $37.0 \%$ & \\
\hline
\end{tabular}


In this study, two definitions were used for true bacteremia: CDC-criteria and the clinical microbiologists' or infectious diseases specialists' judgement. The species distribution between pathogens and contaminants was very similar with both criteria. However, clinical microbiologists and infectious diseases specialists were less likely to regard a strain as contaminant than the CDC-criteria.

Clinicians may be reluctant to not treat a potential pathogen in case of doubt, especially when a patient is severely ill, even when a patient has a confirmed infection at another site with another microorganism that could explain the patients' symptoms. This may result in an overestimation of the total number of actual CoNS bloodstream infections, and thus lead to excess use of antibiotics.

However, we have also shown that in clinical practise many patients will not meet CDCcriteria when having a CoNS bloodstream infection. In $41 \%$ of patients in this study, no more than one blood culture set was drawn. These patients can never meet CDC-criteria, since they require positive blood cultures from more than one set. Also, bloodstream infections with CoNS are often much less severe than with other microorganisms, these patients therefore may not have high fever or hypotension, as well as patients using medication that suppress fever or hypotension. Furthermore, clinicians can use additional patient data in his/her decision making process that are not included in the CDC-criteria, for example a risk factor for acquiring CoNS infection such as indwelling devices. Indeed, in 36 patients in this study, in addition to growing a CoNS from a blood culture, the same CoNS species was cultured from another body site or removed foreign body material, which was deemed clinically relevant in 32 patients. Most of these infections would have been missed when using CDC-criteria only. In these cases, MALDI-TOF MS could be used for comparing the blood culture strain with strains cultured from other materials in order to aid in the diagnosis of CoNS bloodstream infection. Unfortunately, the strains cultured from other body sites were not available for analysis in this study.

Thus, clinicians may overestimate the number of actual CoNS bloodstream infections, but CDC-criteria are also likely to underestimate the number of infections. Additional criteria for diagnosing CoNS bloodstream infections therefore seem necessary. As we have shown, further speciation of CoNS by MALDI-TOF MS could be a useful addition to these criteria. This study suggests that the various CoNS species may vary considerably in pathogenicity. S. schleiferi and S. lugdunensis possess virulence factors, such as extracellular slime which allows them to colonize tissues and evade phagocytosis, enzymes that allow them to invade tissues, and they can bind to a broad variety of molecules [3]. This makes it important to recognise them. Since they express clumping factor or DNAse, it can be difficult to discern them from $S$. aureus. For this, MALDI-TOF MS provides a useful tool. However, in this study of 265 CoNS strains from 245 blood cultures we did not find any of these species, indicating 
that they are rare and using MALDI-TOF MS for finding these species alone would therefore not be very useful.

$S$. saprophyticus is a well-known pathogen in urinary tract infections, but generally these are uncomplicated infections, without bloodstream infection [10]. When found in blood cultures it is most often a contaminant and unrelated to an urinary tract infection [11]. In this study, we found one $S$. saprophyticus strain. Indeed this patient had undergone surgery to the prostate. According to both the clinical microbiologist and CDC-criteria, this patient suffered from a CoNS bloodstream infection. The history of the patient and the CoNS species make it very likely that the focus of this bloodstream infection was the urinary tract.

In this study, the CoNS species most commonly found in bloodstream infections was $S$. epidermidis. However, S. epidermidis was also the most common blood culture contaminant. This was also shown for S. hominis and S. capitis. However, S. haemolyticus was shown to be significantly less likely to be a contaminant than the other species.

For S. haemolyticus, in comparison to S. aureus, S. lugdunensis, S. schleiferi, S. saprophyticus and $S$. epidermidis, much less information on virulence factors is known. $S$. haemolyticus is capable of biofilm formation, which allows it to adhere to foreign material and evade the immune system and antibiotics [12]. Their genome allows for frequent genetic rearrangements, which could contribute to the acquisition of antibiotic resistance genes [13]. Indeed, they show a very high resistance rate for various antibiotics, including heteroresistance to glycopeptides [14-16]. This may result in selection in patients using antibiotics. Also, this makes them very difficult to treat, another reason to recognise them rapidly.

In most other studies, S. epidermidis, S. hominis and S. haemolyticus were also more likely to cause infection than other species [2], although species distribution may vary between countries. For example, in an Indian study by Chaudhury and Kumar [17], S. haemolyticus was the strain most commonly found in blood cultures, however, this study involved only 14 CoNS blood culture isolates in total. Also, in a Japanese study by Kawamura et al. [18] on 1230 clinical CoNS isolates, S. caprae was the third most commonly isolated species, after S. epidermidis and S. haemolyticus. Unfortunately, they did not mention how many strains were isolated from blood cultures and whether they represented an actual infection.

The methods by which CoNS species were identified in all of those studies were not useful in clinical practise, such as sequencing, or the method used to identify them was not very reliable (for example VITEK or API system). MALDI-TOF MS provides a cheap, rapid and reliable alternative for these methods, making it more convenient to use for identification to the species level in the clinical decision making process.

In patients with multiple positive blood culture bottles, it is shown using typing methods that the different bottles contain different strains, thus representing skin flora rather than true infection [19]. These typing methods are helpful in determining whether a patient has a true CoNS infection and prevent overtreatment, but for use in clinical practise they are too time 
and money consuming. In the future however, MALDI-TOF MS might also offer a cheap and fast alternative for these typing methods, so typing may be used in clinical practise, allowing for a more accurate decision making process.

In conclusion, CoNS represent a large group of species, which vary considerably in pathogenicity and determining whether or not a positive blood culture represents a bloodstream infection remains difficult. We have shown in this study that speciation of CoNS could be helpful in establishing whether a patient is suffering from an actual CoNS bloodstream infection. The actual value of identification of CoNS by MALDI-TOF MS in clinical practise remains to be shown in a prospective trial. 


\section{References}

1. Kloos, W.E. and T.L. Bannerman, Update on clinical significance of coagulase-negative staphylococci. Clin Microbiol Rev, 1994. 7(1): p. 117-40.

2. Piette, A. and G. Verschraegen, Role of coagulase-negative staphylococci in human disease. Vet Microbiol, 2009. 134(1-2): p. 45-54.

3. von Eiff, C., G. Peters, and C. Heilmann, Pathogenesis of infections due to coagulasenegative staphylococci. Lancet Infect Dis, 2002. 2(11): p. 677-85.

4. Huebner, J. and D.A. Goldmann, Coagulase-negative staphylococci: role as pathogens. Annu Rev Med, 1999. 50: p. 223-36.

5. Souvenir, D., et al., Blood cultures positive for coagulase-negative staphylococci: antisepsis, pseudobacteremia, and therapy of patients. J Clin Microbiol, 1998. 36(7): p. 1923-6.

6. Loonen, A.J., et al., Comparative study using phenotypic, genotypic, and proteomics methods for identification of coagulase-negative staphylococci. J Clin Microbiol, 2012. 50(4): p. 1437-9.

7. Kim, M., et al., Comparison of the MicroScan, VITEK 2, and Crystal GP with 16S rRNA sequencing and MicroSeq 500 v2.0 analysis for coagulase-negative Staphylococci. BMC Microbiol, 2008. 8: p. 233.

8. Spanu, T., et al., Evaluation of matrix-assisted laser desorption ionization-time-of-flight mass spectrometry in comparison to rpoB gene sequencing for species identification of bloodstream infection staphylococcal isolates. Clin Microbiol Infect, 2011. 17(1): p. 44-9.

9. Dubois, D., et al., Identification of a variety of Staphylococcus species by matrix-assisted laser desorption ionization-time of flight mass spectrometry. J Clin Microbiol, 2010. 48(3): p. 941-5.

10. Ronald, A., The etiology of urinary tract infection: traditional and emerging pathogens. Am J Med, 2002. 113 Suppl 1A: p. 14S-19S.

11. Choi, S.H., et al., Clinical significance of Staphylococcus saprophyticus identified on blood culture in a tertiary care hospital. Diagn Microbiol Infect Dis, 2006. 56(3): p. 337-9.

12. Fredheim, E.G., et al., Biofilm formation by Staphylococcus haemolyticus. J Clin Microbiol, 2009. 47(4): p. 1172-80.

13. Takeuchi, F., et al., Whole-genome sequencing of staphylococcus haemolyticus uncovers the extreme plasticity of its genome and the evolution of human-colonizing staphylococcal species. J Bacteriol, 2005. 187(21): p. 7292-308.

14. Chiew, Y.F., et al., Detection of vancomycin heteroresistant Staphylococcus haemolyticus and vancomycin intermediate resistant Staphylococcus epidermidis by means of vancomycin screening agar. Pathology, 2007. 39(3): p. 375-7.

15. Froggatt, J.W., et al., Antimicrobial resistance in nosocomial isolates of Staphylococcus haemolyticus. Antimicrob Agents Chemother, 1989. 33(4): p. 460-6.

16. Barros, E.M., et al., Staphylococcus haemolyticus as an important hospital pathogen and carrier of methicillin resistance genes. J Clin Microbiol, 2012. 50(1): p. 166-8.

17. Chaudhury, A. and A.G. Kumar, In vitro activity of antimicrobial agents against oxacillin resistant staphylococci with special reference to Staphylococcus haemolyticus. Indian J Med Microbiol, 2007. 25(1): p. 50-2.

18. Kawamura, Y., et al., Distribution of Staphylococcus species among human clinical specimens and emended description of Staphylococcus caprae. J Clin Microbiol, 1998. 36(7): p. 2038-42.

19. Garcia, P., et al., Coagulase-negative staphylococci: clinical, microbiological and molecular features to predict true bacteraemia. J Med Microbiol, 2004. 53(Pt 1): p. 67-72. 



\section{Chapter 6}

An evaluation of three processing methods and the effect of reduced culture times for faster direct identification of pathogens from BacT/ALERT blood cultures by MALDI-TOF MS

\section{Anne J.M. Loonen ${ }^{1-4}$, Arjan R. Jansz' ${ }^{1}$, Jitske Stalpers ${ }^{1}$, Petra}

F.G. Wolffs ${ }^{3}$, and Adriaan J.C. van den Brule B $^{1,2,4}$

1 PAMM Laboratories, Department of Medical Microbiology, Veldhoven/Eindhoven, The Netherlands ${ }^{2}$ Fontys University of Applied Science, Department of Medical Molecular Diagnostics, Eindhoven, The Netherlands ${ }^{3}$ Maastricht University Medical Centre, CAPHRI, Department of Medical Microbiology, Maastricht, The Netherlands ${ }^{4}$ Jeroen Bosch Hospital, Laboratory for Molecular Diagnostics, Departments of Medical Microbiology and Pathology, 's-Hertogenbosch, The Netherlands 8 Eur J Clin Microbiol Infect Dis 2012, Jul;31(7):1575-83

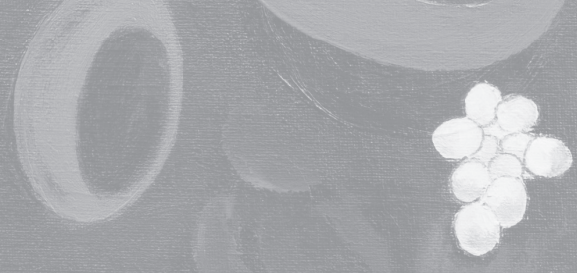




\section{Abstract}

Matrix-assisted laser desorption ionisation time-of-flight mass spectrometry (MALDI-TOF MS) is a fast and reliable method for the identification of bacteria from agar media. Direct identification from positive blood cultures should decrease the time to obtaining the result. In this study, three different processing methods for the rapid direct identification of bacteria from positive blood culture bottles were compared. In total, 101 positive aerobe BacT/ALERT bottles were included in this study. Aliquots from all bottles were used for three bacterial processing methods, i.e. the commercially available Bruker's MALDI Sepsityper kit, the commercially available Molzym's MolYsis Basic 5 kit and a centrifugation/washing method. In addition, the best method was used to evaluate the possibility of MALDI application after a reduced incubation time of $7 \mathrm{~h}$ of Staphylococcus aureus- and Escherichia coli -spiked (1.000, 100 and 10 colony-forming units [CFU]) aerobe BacT/ALERT blood cultures.

Sixty-six (65\%), $51(50.5 \%)$ and $79(78 \%)$ bottles were identified correctly at the species level when the centrifugation/washing method, MolYsis Basic 5 and Sepsityper were used, respectively. Incorrect identification was obtained in 35 (35\%), 50 (49.5\%) and 22 (22\%) bottles, respectively. Gram-positive cocci were correctly identified in 33/52 (64\%) of the cases. However, Gram-negative rods showed a correct identification in 45/47 (96\%) of all bottles when the Sepsityper kit was used. Seven hours of pre-incubation of $S$. aureus- and E. coli -spiked aerobe BacT/ALERT blood cultures never resulted in reliable identification with MALDI-TOF MS.

Sepsityper is superior for the direct identification of microorganisms from aerobe BacT/ ALERT bottles. Gram-negative pathogens show better results compared to Gram-positive bacteria. Reduced incubation followed by MALDI-TOF MS did not result in faster reliable identification. 


\section{Introduction}

Fast and accurate diagnostic methods are necessary in order to be able to initiate correct treatment for bloodstream infections. Adequate and fast antibiotic treatment reduces the mortality rates in these patients [1,2]. Cultivation techniques take at least a day, and although the subsequent Gram staining can be performed in several minutes, the result from this staining technique alone does often not provide sufficient information to administer appropriate antibiotic therapy. Therefore, additional tests have to be performed so as to identify the pathogen that causes the disease, leading to an increased time to initiate appropriate therapy.

Improved techniques have been developed to reduce the time-to-result, i.e. VITEK 2, FISH (fluorescent in situ hybridisation) and (commercial) real-time polymerase chain reaction (PCR)- based methods [3-6]. However, most of these techniques are limited by high costs and the fact that not every species can be detected in one assay. This makes it difficult to implement the technique in routine diagnostics.

Recently, a new method became available in several clinical microbiology laboratories, i.e. matrix-assisted laser desorption ionisation time-of-flight mass spectrometry (MALDITOF MS). This technique can be used for pathogen identification from agar media [7-9]. However, several reports are currently available which describe the possibility of direct identification from positive blood cultures [10-15]. Most reports describe the use of BACTEC bottles, but a few studies $[16,17]$ used BacT/ALERT bottles. This makes the BacT/ALERT system underrepresented in the literature, while it is a frequently used culture system in many laboratories. Pathogens from blood cultures need to be separated from the liquid culture media prior to detection by MALDI-TOF MS. Several similar protocols have been published consisting either of the removal of erythrocytes by centrifugation [11,12] or serum separation tubes [10,14], followed by subsequently inactivating the bacteria by using ethanol, protein precipitation and extraction by formic acid and acetonitrile.

Recently, new processing methods have become available. Sepsityper (Bruker Daltonics) allows the easy and fast identification of bacteria from positive blood cultures by using MALDI-TOF MS. Another commercially available method, MolYsis (Molzym), is capable of removing human background (cells and DNA) from blood (culture) samples by selective lysis and, additionally, ensures pathogen enrichment. This method has been shown to be suitable for molecular diagnostics $[4,18]$. However, it is still unknown as to whether this enrichment method is also feasible for direct pathogen identification from positive blood cultures by MALDI-TOF MS.

In addition, it was demonstrated that accelerated detection of staphylococci from spiked blood cultures was possible after only $5 \mathrm{~h}$ of pre-incubation when, subsequently, MolYsis enrichment was combined with real-time PCR [4]. 
In the present study, we compared three easy-to-perform protocols, i.e. commercially available MolYsis Basic 5 and Sepsityper, and a non-commercial centrifugation/washing method as described by Ferreira et al. [11], for the direct identification of bacteria from positive aerobe BacT/ALERT blood cultures with MALDI-TOF MS. The results were compared to conventional diagnostics and MALDI-TOF MS directly from blood agar. To evaluate the possibility of reduced time-to-identification from blood cultures when using MALDI-TOF MS analysis, the best processing method was also investigated in relation to the reduced incubation times (7 h) of Staphylococcus aureus- and Escherichia coli-spiked BacT/ALERT blood cultures.

\section{Materials and Methods}

\section{Clinical samples}

Blood samples (8-10 ml) from patients were collected in standard aerobe (SA) and anaerobe (SN) BacT/ALERT blood culture bottles (bioMérieux, Marcy L'Etoile, France) without charcoal, and handled according to the manufacturer's instructions. A total of 101 positive aerobe blood cultures (equal distribution of Gram-negative rods and Gram-positive cocci) were collected for this study (see Table 1 for details).

When patient samples were detected as positive by the BacT/ALERT 3D system (bioMérieux, Marcy L'Etoile, France), Gram staining was performed and, based on the Gram result, additional subculture and biochemical tests were performed (conventional diagnostics as schematically shown in Figure 3a). When Gram-negative rods were detected by Gram staining, several agar media were inoculated for subculture. One additional blood plate was inoculated for a spot-subculture (concentrated spot with blood culture material in the centre of the plate), which was used for the MALDI-TOF MS analysis. Resistance was determined by the use of VITEK 2 (bioMérieux). When Gram-positive cocci were detected in the blood culture sample, several agar media were inoculated for subculture. Standard biochemical tests were performed (based on the Gram result), because MALDI-TOF MS was still being validated in the laboratory for Gram-positive cultures, to obtain identification of the pathogen (coagulase, TLM plate, BAGG test, tellurite plate, rapid strep test, VITEK 2 [CoNS species]). As Streptococcus pneumoniae can die in the culture bottle (self-lysing ability), an LytA realtime PCR [19] was performed when no subculture on blood agar was detected. Resistance determination occurred by the use of the disk diffusion method.

One colony of the subculture on blood agar was used for MALDI-TOF MS.

The Internal Review Board of the hospital ethically approves the anonymous use of remaining patient material. 


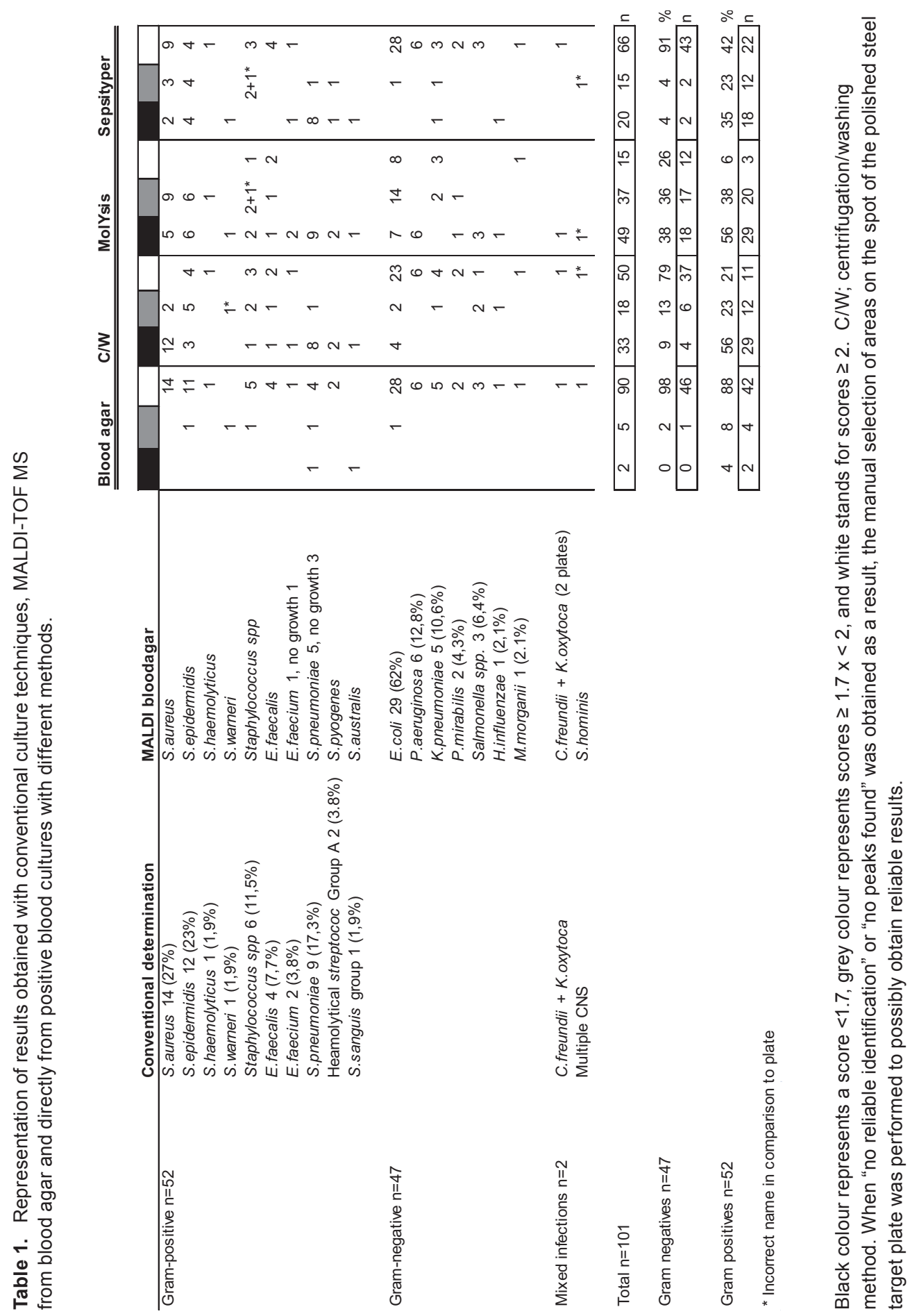




\section{Strains for spiking experiments}

The blood of healthy human volunteers $(10 \mathrm{ml})$ was collected in SA BacT/ALERT culture bottles. For spiking experiments, S. aureus (ATCC 25923) and E. coli (ATCC 25922) were used.

\section{Processing methods for MALDI-TOF MS}

From each positive culture bottle, three aliquots were taken; for clarification, see Figure 1. For the Sepsityper method (Bruker Daltonics $\mathrm{GmbH}$, Bremen, Germany) the recommended quantity of $1 \mathrm{ml}$ was used. The protocol was performed as recommended by the manufacturer. For the MolYsis Basic method (Molzym GmbH, Bremen, Germany), $1 \mathrm{ml}$ was used and the provided protocol was followed until the first washing step. For the third method, $4 \mathrm{ml}$ of positive culture material was used. The centrifugation/washing method was performed as described by Ferreira et al. [11]. All methods resulted in a pellet, which was subsequently dissolved in $300 \mu \mathrm{l}$ of water, to which $900 \mu \mathrm{l}$ of ethanol absolute was added. The mixture was centrifuged at 14,000 rpm for $2 \mathrm{~min}$, and the supernatant was discarded. The centrifugation step was repeated so as to remove all residual supernatant. The pellet size was estimated in $\mu \mathrm{l}$ and, to this pellet, in a $1: 1$ ratio, $70 \%$ formic acid was added and mixed thoroughly. Next, the same amount of acetonitrile was added (1:1 ratio with formic acid) and mixed well before centrifugation at $14,000 \mathrm{rpm}$ for $2 \mathrm{~min}$. One $\mu \mathrm{l}$ of the supernatant was applied on a spot of the polished steel target plate and air-dried at room temperature. The dried spot was overlaid with $1 \mu \mathrm{l}$ of HCCA matrix (Bruker Daltonics $\mathrm{GmbH}$, Bremen, Germany) solution (saturated alpha-cyano-4-hydroxycinnamic acid in 50\% acetonitrile and $2.5 \%$ trifluoroacetic acid) and allowed to air-dry at room temperature.

\section{MALDI-TOF MS analysis}

Samples were placed in the MALDI-TOF MS spectrometer (BioTyper, Bruker Daltonics $\mathrm{GmbH}$, Bremen, Germany). The spectrometer was calibrated with Bruker bacterial test standard (Bruker Daltonics $\mathrm{GmbH}$, Bremen, Germany). For pathogen identification, 240 shots in 40-shot steps from different positions of the target spot (automatic) were recorded to obtain a spectrum per strain and spectra were imported in the MALDI BioTyper 2.0 software (Bruker Daltonics $\mathrm{GmbH}$, Bremen, Germany) and analysed by standard pattern matching; the BioTyper database version V3 1.1.0_3476-3740 was used as a reference. No intervention took place in the process from measurement to identification. For each strain, the highest score obtained by pattern matching to the database was used for the identification. Scores $<1.7$ were considered to be unreliable, scores $\geq 1.7 x<2$ were considered to be reliable to the genus level; however, if database match number 1 and 2 resulted in the same strain name, it was considered correct to the species level, and scores $\geq 2$ were considered to be reliable to the species level. When "no reliable identification" or "no peaks found" was obtained 
as a result, the manual selection of areas on the spot of the polished steel target plate was performed to possibly obtain reliable results. All data were compared to conventional diagnostics and MALDI-TOF MS results obtained from a colony grown on blood agar.

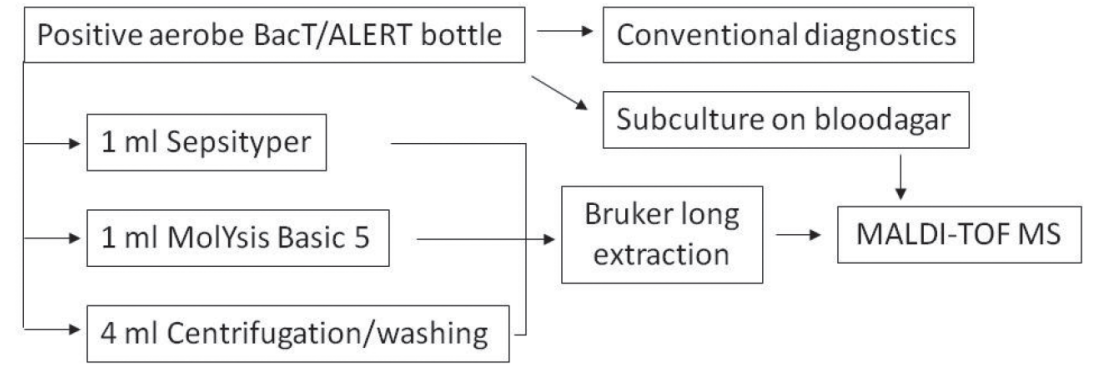

Figure 1. Overview of bacterial identification from positive aerobe BacT/ALERT cultures by using MALDI-TOF MS.

Three aliquots were taken from each positive blood culture bottle to analyse the different processing methods. All methods were performed as described in the Materials and Methods section. All results were compared to conventional diagnostics and/or MALDI-TOF MS directly from blood agar.

\section{Statistical analysis}

For analysis of the results, the McNemar test was used. The total amount of correct identifications (scores $\geq 1.7$ with hit 1 and 2 being similar, and scores $>2$ ) and incorrect identifications were compared for all of the methods investigated. A binomial distribution was used.

\section{Results}

One hundred and one aerobe blood cultures detected as positive by the BacT/ALERT 3D system were processed by conventional methods, tested by MALDI-TOF MS from blood agar (direct smear method) and tested directly from positive blood cultures by using three different bacterial isolation methods (Figure 1). Only aerobe BacT/ALERT bottles were used because, in pilot experiments (preliminary data were obtained with small sample numbers), anaerobe BacT/ALERT bottles frequently resulted in unreliable data. Fifty- two cultures showed the presence of Gram-positive bacteria after Gram staining, 47 cultures showed the presence of Gram-negative bacteria and two 2 cultures indicated, after conventional diagnostics, the presence of multiple bacterial species (Table 1). The direct smear method (blood agar) is the standard diagnostic method for Gram-negative bacteria and resulted in reliable identification. For the Gram-positive cultures, both conventional diagnostics and the direct smear method 
were performed (due to the ongoing process validation of MALDI-TOF MS diagnostics). Four of 52 blood agar plates did not result in growth. Of these four, three were characterised as $S$. pneumoniae by PCR and one was plated for a second time and was identified as Enterococcus faecium by conventional diagnostics. S. sanguis was detected as S. australis by MALDI-TOF MS from blood agar. Blood culture samples that resulted in "unreliable data" or "no peaks found" were retested immediately from the same spot by the manual selection of areas on the polished steel target plate. This procedure never resulted in acceptable results $(\geq 1.7)$ for further analysis.

Of the methods used for direct identification from positive blood cultures, Sepsityper showed (Table 1 and Figure 2) a correct identification in 79/101 cultures $(\geq 1.7)$ equal to $78 \%$, in contrast to MolYsis and the centrifugation/washing method, where only $50.5 \%$ and $65 \%$ were correctly identified, respectively. However, when Gram-negative cultures were separated from Gram-positive cultures and mixed cultures, it can be seen that cultures containing Gramnegatives show more correct identifications with all of the methods used. When Sepsityper was used, 96\% (45/47) of all Gram-negative cultures showed correct identification ( $\geq 1.7)$, on the contrary to cultures containing Gram-positives, where only $64 \%$ of the results $(33 / 52)$ were correct. For the centrifugation method, 92\% (43/47) and for the MolYsis method, 62\% $(29 / 47)$ of the Gram negatives were identified correctly $(\geq 1.7)$. With both the centrifugation and the MolYsis methods, $42 \%$ (22/52) of the Gram-positive cultures were correctly identified. Staphylococci were most frequently detected in the cultures containing Gram-positive bacteria, i.e. they represented $65 \%$ of these bottles (34/52). The direct smear method always resulted in reliable identification by MALDI-TOF MS. When identification directly from the positive blood culture was performed, Sepsityper resulted in the most correct identifications, as $12 / 14$ S. aureus, $8 / 12$ S. epidermidis, $1 / 1 \mathrm{~S}$. haemolyticus, $0 / 1 \mathrm{~S}$. warneri and $5 / 6$ Staphylococcus spp. showed identical results compared to the results obtained from the blood agar plate. In this study, streptococci showed to be the second most detected group of bacteria from culture bottles, with $12 / 52(23 \%)$ cases. S. pneumoniae was recovered most frequently, in 9/12 cases. In three cases, no growth was detected on blood agar and, therefore, the direct smear method could not be performed. In five cases, S. pneumoniae was identified correctly from blood agar ( $\geq 1.7$ ); however, directly from positive cultures successful detection of this pathogen proved to be difficult, as correct identification only occurred once with both the centrifugation/washing method and the Sepsityper method $(\geq 1.7 x<2)$. Enterococci were recovered from $6 / 52$ cultures (11.5\%); in four cases $E$. faecalis was found and in two cases, E. faecium was found. Enterococci were detected accurately $(>2)$ from blood agar (Table 1), except for one E. faecium strain which did not result in proper growth. Sepsityper characterised all $E$. faecalis cultures and one $E$. faecium culture accurately $(>2)$.

The McNemar test was used to investigate the statistical significance of the obtained results. From the analysis of the total amount of blood cultures tested, it was shown that the direct 


\section{A}
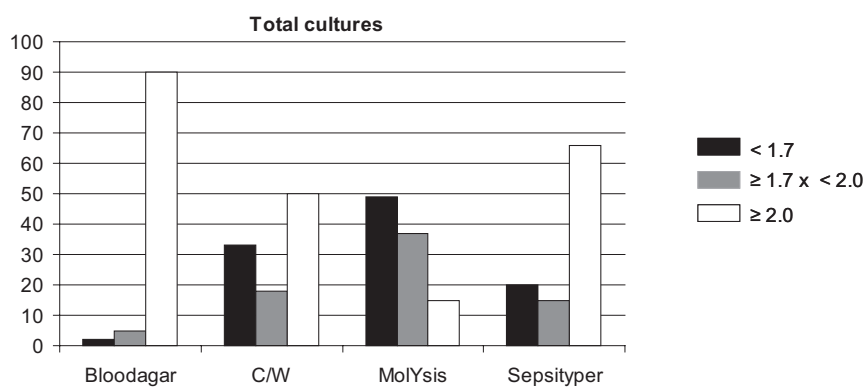

B
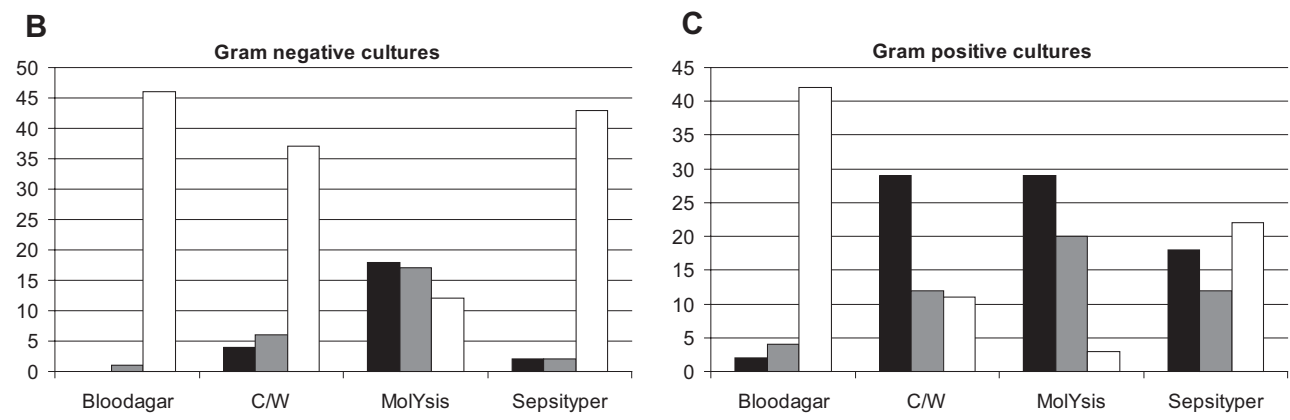

Figure 2. Overview of results obtained with different bacterial isolation methods from positive blood cultures in comparison to results obtained with the direct smear method directly from blood agar.

a The overall results are depicted obtained after the analysis of all bottles included in this study. $b$ Specific results obtained from the 47 bottles containing Gram-negative bacteria. $c$ The data of the 52 bottles in which Gram-positive bacteria were found are shown. C/W; centrifugation/washing method. Statistical significance was analysed by using the McNemar test, see the Results section for details.

smear method always showed significantly better results compared to identification directly from positive blood cultures (all methods), $p \leq 0.001$. The Sepsityper method showed to lead to significantly better results compared to the MolYsis enrichment, $p=0.000$. No significant difference was observed between the $\mathrm{C} / \mathrm{W}$ method and MolYsis $(p=0.11)$ and the $\mathrm{C} / \mathrm{W}$ method and Sepsityper $(p=0.19)$. When only the blood cultures containing Gram positives were analysed, by using the McNemar test, it was again shown that the direct smear method was always significantly better compared to analysis directly from blood cultures, $p \leq 0.002$. Sepsityper proved to be significantly better compared to the MolYsis treatment, $p=0.008$. The C/W method was comparable to Sepsityper and MolYsis. As the direct smear method for all Gram-negative-containing blood cultures always resulted in correct identification, these results were not used for McNemar analysis. The C/W method and the Sepsityper method performed on positive blood cultures (Gram-negative bacteria) lead to significantly better results compared to MolYsis ( $p \leq 0.01$ ), but not when compared to each other. 
The time needed to process positive cultures with the described methods varied. Sepsityper was fast (20 min) and easy to use, while MolYsis (50 min) had quite some incubation steps, took a lot of hands-on time and did not lead to reliable results. The centrifugation method is a good alternative (30 min), especially for Gram-negative pathogens, as it is cheap to use and shows promising results for this group of microorganisms. In contrast, the commercially available methods are more expensive (the list price in The Netherlands for the Sepsityper and Molzym methods are 4 and 8 Euros per test, respectively).

In preliminary experiments, BacT/ALERT bottles with charcoal were also analysed by using the Sepsityper method (Bruker) and the method described by Moussaoui et al. [14], using serum separation tubes. Bottles containing charcoal never showed reliable results and mostly resulted in "no peaks found", as the charcoal was always isolated with the bacteria from the positive cultures.

\section{Spiked aerobe cultures with reduced incubation times}

To decrease the time-to-result even more, aerobe BacT/ALERT blood culture bottles were spiked with approximately $1.000,100$, and 10 colony forming units (CFU) of $S$. aureus (ATCC 25923) or E. coli (ATCC 25922) per bottle (representative of 100,10 and $1 \mathrm{CFU} / \mathrm{ml}$ blood) and were incubated for $7 \mathrm{~h}$ in order to be able to obtain results within one working day $(8 \mathrm{~h})$. Sepsityper was used in combination with MALDI-TOF MS for bacterial identification from these bottles, as this method proved to be superior in previous experiments. After $7 \mathrm{~h}$ of incubation in the BacT/ALERT 3D system, a 1-ml aliquot was taken from each bottle and processed as described in the Sepsityper manual. The culture bottles were placed back in the BacT/ALERT 3D system to determine the time to positivity for each bottle and to use these bottles as positive controls for the Sepsityper procedure the next day. All experiments were performed three times (independently). Reduced incubation times of $7 \mathrm{~h}$ did not result in a peak pattern; no peaks were detected even after the manual selection of areas on the target plate. The $S$. aureus-spiked bottles were flagged positive after $16 \mathrm{~h}$ (1.000 CFU), $17.5 \mathrm{~h}$ (100 CFU) and 19.5 $\mathrm{h}$ (10 CFU) by the BacT/ALERT 3D system, and did show correct identification $(\geq 1.7 \mathrm{x}<2$ or $>2$ ) of $S$. aureus with the Sepsityper method. The $E$. coli-spiked cultures were flagged positive after $12 \mathrm{~h}$ (1.000 CFU), $13 \mathrm{~h}$ (100 CFU), and $14 \mathrm{~h}$ (10 CFU) by the BacT/ALERT 3D system, and were correctly identified (>2) as containing E. coli by MALDI-TOF MS analysis. As $1 \mathrm{ml}$ might be a low amount of material to analyse after only $7 \mathrm{~h}$ of incubation, it was hypothesised that an increase in the sample volume might lead to the reliable identification of $S$. aureus-spiked aerobe cultures; therefore, the centrifugation/washing method $(4 \mathrm{ml})$ was tested as well as the Sepsityper method, for which $2 \mathrm{ml}$ was used (the chemicals from the kit were increased in the same ratio). Again, for both methods, no reliable identification was found as a result, but the positive bottles did show the reliable identification of $S$. aureus the next day $(\geq 1.7 x<2$ or $>2$ ). As a last effort, a new protocol was investigated which was published by Moussaoui et al. 
[14] and describes the use of serum separation tubes (Becton Dickinson). This protocol was optimised for $3.5 \mathrm{ml}$ of culture material and combined with the saponin pre-treatment step as described by Lupetti et al. [20]. The results indicated a non-reliable identification after $7 \mathrm{~h}$ of incubation for $S$. aureus-spiked cultures. The positive bottles did result in reliable identification $(\geq 1.7 \times<2)$ the next day.

\section{Discussion}

In this study, Sepsityper was shown to be the best method for positive aerobe BacT/ALERT blood cultures as 79/101 cultures were correctly identified (78\%). By using the MolYsis Basic (Molzym) method, $50.5 \%$ of all bottles were correctly identified, and the centrifugation/ washing method according to Ferreira et al. [11] resulted in a correct identification rate of $65 \%$ of all cultures. In the current literature, a correct identification rate of between 30 and $90 \%$ is described $[10,12,14,15,17]$, and this is in agreement with the data from this study. Christner et al. [15] and Moussaoui et al. [14] both describe high correct identification rates of approximately $90 \%$, which is higher than the $78 \%$ found in this study. The most important difference is the use of BACTEC cultures (compared to BacT/ALERT cultures) in those studies. In addition, a clear difference was observed between the identification of Gram-negative versus Gram-positive bacteria, $96 \%$ versus 66\%, respectively (Sepsityper), which confirms the observations of previous studies [11, 12].

Streptococci were found to be problematic pathogens for MALDI-TOF MS identification directly from positive cultures. In three cases, $S$. pneumoniae had to be detected by LytA real-time PCR, because no growth was observed on agar media, as the bacteria died in the bottles. In these three specific cases, no reliable identification from the positive cultures was possible with all of the tested methods. For the other bottles containing streptococci $(n=9)$, the direct identification from blood culture material resulted mostly in unreliable identification $(<1.7)$ as well, while the identification from agar media was not problematic for the majority (7/9 cases). The problems encountered in this study have also been observed by others [12]. In pilot experiments, it was found that anaerobe bottles more frequently resulted in unreliable data. Therefore, in this study, only positive aerobe BacT/ALERT cultures were used. The reason for the better performance of aerobe cultures might be found in the fact that the most commonly detected bacteria grow better in aerobe culture circumstances. This can, subsequently, result in a higher bacterial load in aerobe culture bottles and, subsequently in better MALDI-TOF MS spectra. Further studies should focus on the usage of anaerobe BacT/ALERT blood cultures for the optimal implementation of MALDI-TOF MS in the clinical microbiology laboratory. In contrast, for BACTEC bottles, several groups have described similar results for both positive aerobe and anaerobe cultures [10,15]. This observation needs to be properly addressed in the future. 
Previously, we showed the possibility of reduced incubation times for blood cultures spiked with staphylococci $(1 \mathrm{CFU} / \mathrm{ml}$ blood); only $5 \mathrm{~h}$ of pre-incubation was sufficient to detect staphylococci when using real-time PCR as an analytical tool [4]. In this study, a pre-incubation of $7 \mathrm{~h}$ was investigated by using $S$. aureus-and E. coli-spiked BacT/ALERT bottles for subsequent rapid direct analysis with MALDI-TOF MS. Several approaches were undertaken but these never resulted in reliable results. It has been described that at least $>10^{8} \mathrm{CFU} / \mathrm{ml}$ need to be present in culture material in order to obtain a spectra that resembles that from a pure culture, and at $10^{6} \mathrm{CFU} / \mathrm{ml}$, the spectra resembles that of a sterile culture [15]. This can be the explanation for the results obtained in this study. It remains unexplained why unreliable data have been obtained when $4 \mathrm{ml}$ was used after $7 \mathrm{~h}$ of incubation.

In this study, S. aureus-containing cultures were never misidentified as CoNS and vice versa. Ferroni et al,[16] showed similar results with positive BacT/ALERT bottles concerning staphylococci. This may contribute to the discrimination of contamination versus true infection. Another positive effect of MALDI-TOF MS identification is that CoNS are now more frequently identified to the species level. This group of microorganisms is increasingly described in the literature in relation to infection [21, 22], making it clinically relevant to investigate this group of pathogens in more detail.

Positive BacT/ALERT bottles were tested immediately in the morning (9 am). If bottles became positive during the night in the BacT/ALERT 3D system, they remained in the culture machine until $8.30 \mathrm{am}$, and the time to positivity (TTP) was not registered for this study. Bottles were kept at room temperature, when positive, between 8.30 am and 6 pm (working hours); subsequently, they were stored for 1 week at $35^{\circ} \mathrm{C}$. Bottles that became positive in the afternoon were tested the next day. However, if only Sepsityper is used for the analysis, positive bottles can be collected several times a day; for example every $2 \mathrm{~h}$, starting at 8.30 am, with the last round at $5.00 \mathrm{pm}$.

The results of this study implicate that routine diagnostics can be optimised when positive blood cultures can be processed by Sepsityper followed by MALDI-TOF MS, as schematically represented in Figure 3b. Pathogen identification can be available within 20 min (depending on the number of positive cultures that need to be processed), in contrast to the current workflow (approximately 1-2 days; see Figure 3a). This rapid direct identification of microorganisms from positive cultures can have a major impact in the clinic. Patient-tailored treatment decisions can be made more rapidly, but the final antibiotic therapy can only be installed if antibiotic susceptibility results are available. Although Sepsityper was superior in this study, alternatives to this approach exist and clinical microbiology laboratories with a lower budget might implement the centrifugation/washing method for positive cultures containing Gramnegative pathogens and apply the Sepsityper method only for positive cultures containing Gram-positive pathogens. Another alternative might be the usage of the spot subculture agar plates (materials and methods) for cultures which became positive early in the morning and 
A

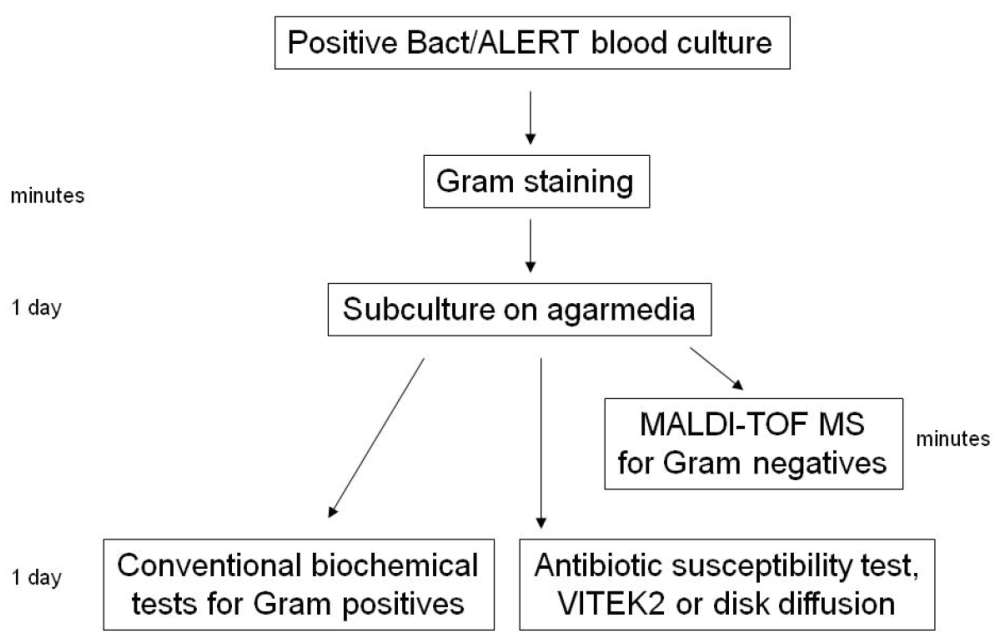

B

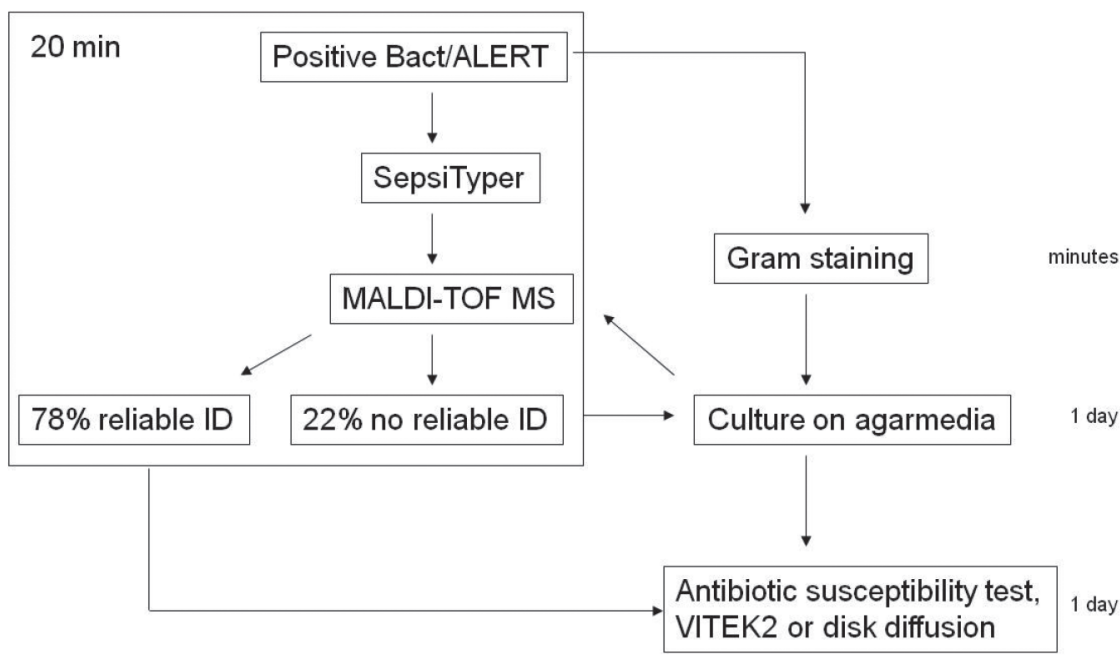

Figure 3. Schematic representation of the current (a) and possible new diagnostic workflow (b). Every positive culture (anaerobe cultures need further research) will be processed by Sepsityper and analysed by using MALDI-TOF MS to have optimal speed for the identification of pathogens. Additionally, Gram staining and subculture on agar media will be started for antibiotic susceptibility testing and, when necessary, these agar media can be used for MALDI-TOF MS when the direct identification from cultures is unsuccessful. 
Sepsityper for cultures that became positive later during the day. At the end of the working day, these spot subcultures contain enough bacteria for MALDI-TOF MS analysis via the direct smear method. Unfortunately, it is still not possible to use MALDI-TOF MS for antibiotic susceptibility testing or for detection of virulence factors. Additional studies are needed for this. In summary, after the analysis of more than 100 positive aerobe BacT/ALERT cultures with three different processing methods, it can be concluded that Sepsityper is superior for the detection of pathogens directly from positive aerobe BacT/ALERT cultures by MALDI-TOF MS in comparison to processing cultures by MolYsis or the centrifugation/washing method. Gram-negative pathogens are easier to identify from cultures compared to Gram-positive microorganisms. Reduced incubation ( $7 \mathrm{~h}$ ) of $S$. aureus-and E. coli-spiked aerobe BacT/ ALERT cultures did not result in reliable identification with all of the methods tested.

\section{Acknowledgements}

We thank Wouter Bolderdijk for the technical assistance, and Lieke Wielders, M.Sc. for her valuable contribution to this manuscript. 


\section{References}

1. Kumar, A., et al., Initiation of inappropriate antimicrobial therapy results in a fivefold reduction of survival in human septic shock. Chest, 2009. 136(5): p. 1237-48.

2. Kumar, A., et al., Duration of hypotension before initiation of effective antimicrobial therapy is the critical determinant of survival in human septic shock. Crit Care Med, 2006. 34(6): p. 158996.

3. Peters, R.P., et al., Rapid identification of pathogens in blood cultures with a modified fluorescence in situ hybridization assay. J Clin Microbiol, 2006. 44(11): p. 4186-8.

4. Loonen, A.J., et al., Acceleration of the direct identification of Staphylococcus aureus versus coagulase-negative staphylococci from blood culture material: a comparison of six bacterial DNA extraction methods. Eur J Clin Microbiol Infect Dis, 2011. 30(3): p. 337-42.

5. Wallet, F., et al., Preliminary clinical study using a multiplex real-time PCR test for the detection of bacterial and fungal DNA directly in blood. Clin Microbiol Infect, 2010. 16(6): $p$. 774-779.

6. Wellinghausen, N., et al., Diagnosis of bacteremia in whole blood samples by a commercial universal 16S rDNA-based PCR and sequence analysis. J Clin Microbiol, 2009. 47(9): p. 2759-2765.

7. van Veen, S.Q., E.C. Claas, and E.J. Kuijper, High-throughput identification of bacteria and yeast by matrix-assisted laser desorption ionization-time of flight mass spectrometry in conventional medical microbiology laboratories. J Clin Microbiol, 2010. 48(3): p. 900-7.

8. Sogawa, K., et al., Use of the MALDI BioTyper system with MALDI-TOF mass spectrometry for rapid identification of microorganisms. Anal Bioanal Chem, 2011. 400(7): p. 1905-11.

9. Bizzini, A., et al., Matrix-assisted laser desorption ionization-time of flight mass spectrometry as an alternative to $16 S$ rRNA gene sequencing for identification of difficult-to-identify bacterial strains. J Clin Microbiol, 2011. 49(2): p. 693-6.

10. Stevenson, L.G., S.K. Drake, and P.R. Murray, Rapid Identification of Bacteria in Positive Blood Culture Broths by MALDI-TOF Mass Spectrometry. J Clin Microbiol, 2010. 48(2): p. 4447.

11. Ferreira, L., et al., Microorganisms Direct Identification from Blood Culture by Maldi-Tof Mass Spectrometry. Clin Microbiol Infect, 2011. 17(4): p. 546-551.

12. La Scola, B. and D. Raoult, Direct identification of bacteria in positive blood culture bottles by matrix-assisted laser desorption ionisation time-of-flight mass spectrometry. PLoS One, 2009. 4(11): p. e8041.

13. Prod'hom, G., et al., Matrix-assisted laser desorption ionization-time of flight mass spectrometry for direct bacterial identification from positive blood culture pellets. J Clin Microbiol, 2010. 48(4): p. 1481-3.

14. Moussaoui, W., et al., Matrix-assisted laser desorption ionization time-of-flight mass spectrometry identifies $90 \%$ of bacteria directly from blood culture vials. Clin Microbiol Infect, 2010. 16(11): p. 1631-8.

15. Christner, M., et al., Rapid identification of bacteria from positive blood culture bottles by use of matrix-assisted laser desorption-ionization time of flight mass spectrometry fingerprinting. $\mathrm{J}$ Clin Microbiol, 2010. 48(5): p. 1584-91.

16. Ferroni, A., et al., Real-time identification of bacteria and Candida species in positive blood culture broths by matrix-assisted laser desorption ionization-time of flight mass spectrometry. J Clin Microbiol, 2010. 48(5): p. 1542-8.

17. Szabados, F., et al., The sensitivity of direct identification from positive BacT/ALERT (bioMerieux) blood culture bottles by matrix-assisted laser desorption ionization time-of-flight mass spectrometry is low. Clin Microbiol Infect, 2011. 17(2): p. 192-195.

18. Hansen, W.L., C.A. Bruggeman, and P.F. Wolffs, Evaluation of new preanalysis sample treatment tools and DNA isolation protocols to improve bacterial pathogen detection in whole blood. J Clin Microbiol, 2009. 47(8): p. 2629-31.

19. McAvin, J.C., et al., Sensitive and specific method for rapid identification of Streptococcus pneumoniae using real-time fluorescence PCR. J Clin Microbiol, 2001. 39(10): p. 3446-51. 
20. Lupetti, A., et al., Rapid identification and antimicrobial susceptibility profiling of Gram-positive cocci in blood cultures with the Vitek 2 system. Eur J Clin Microbiol Infect Dis, 2010. 29(1): p. 89-95.

21. Piette, A. and G. Verschraegen, Role of coagulase-negative staphylococci in human disease. Vet Microbiol, 2009. 134(1-2): p. 45-54.

22. von Eiff, C., G. Peters, and C. Heilmann, Pathogenesis of infections due to coagulasenegative staphylococci. Lancet Infect Dis, 2002. 2(11): p. 677-85. 


\section{Chapter 7}

Comparison of pathogen DNA isolation methods from large

volumes of whole blood to improve molecular diagnosis of

bloodstream infections

Anne J.M. Loonen ${ }^{12}$, Martine P. Bos ${ }^{3}$, Bart van Meerbergen ${ }^{4}$, Sigi Neerken ${ }^{5}$,

Arnold Catsburg ${ }^{3}$, Irene Dobbelaer ${ }^{5}$, Roel Penterman ${ }^{4}$, Geert Maertens $^{4}$,

Paul van de Wiel $^{5}$, Paul Savelkoul ${ }^{3}$, and Adriaan J.C. van den Brule ${ }^{1,2}$

Jeroen Bosch Hospital, Department of Molecular Diagnostics, 's-Hertogenbosch, The Netherlands ${ }^{2}$ Fontys University of Applied Sciences, Department of Medical Molecular Diagnostics, Eindhoven, The Netherlands

${ }^{3}$ VU University Medical Center, Department of Medical Microbiology and Infection Control, Amsterdam and

Microbiome Ltd., Houten, The Netherlands

${ }^{4}$ Biocartis NV, Mechelen, Belgium ${ }^{5}$ Philips Research, Department of Molecular Diagnostics, Eindhoven, The Netherlands

\section{PLOS ONE 2013, 8:e72349}




\section{Abstract}

For patients suffering from bloodstream infections (BSI) molecular diagnostics from whole blood holds promise to provide fast and adequate treatment. However, this approach is hampered by the need of large blood volumes.

Three methods for pathogen DNA isolation from whole blood were compared, i.e. an enzymatic method (MolYsis, 1-5 ml), the novel non-enzymatic procedure (Polaris, 1-5 ml), and a method that does not entail removal of human DNA (Triton-Tris-EDTA EasyMAG, $200 \mu \mathrm{l})$. These methods were evaluated by processing blood spiked with $0-1000 \mathrm{CFU} / \mathrm{ml}$ of Staphylococcus aureus, Pseudomonas aeruginosa and Candida albicans. Downstream detection was performed with real-time PCR assays.

Polaris and MolYsis processing followed by real-time PCRs enabled pathogen detection at clinically relevant concentrations of 1-10 CFU/ml blood. By increasing sample volumes, concurrent lower cycle threshold $(\mathrm{Ct})$ values were obtained at clinically relevant pathogen concentrations, demonstrating the benefit of using larger blood volumes. A 100\% detection rate at a concentration of $10 \mathrm{CFU} / \mathrm{ml}$ for all tested pathogens was obtained with the Polaris enrichment, whereas comparatively lower detection rates were measured for MolYsis (50$67 \%$ ) and EasyMAG (58-79\%). For the samples with a concentration of $1 \mathrm{CFU} / \mathrm{ml}$ Polaris resulted in most optimal detection rates of $70-75 \%$ (MolYsis $17-50 \%$ and TTE-EasyMAG 20-36\%). The Polaris method was more reproducible, less labour intensive, and faster (45 minutes (including Qiagen DNA extraction) vs. 2 hours (MolYsis)).

In conclusion, Polaris and MolYsis enrichment followed by DNA isolation and real-time PCR enables reliable and sensitive detection of bacteria and fungi from $5 \mathrm{ml}$ blood. With Polaris results are available within 3 hours, showing potential for improved BSI diagnostics. 


\section{Introduction}

Bloodstream infections (BSI) can be caused by a wide variety of pathogens and remain a significant cause of morbidity and mortality especially in the Intensive Care Unit [1-3]. This could be significantly improved by pathogen-tailored antibiotic and antifungal treatment [4]. This requires a fast identification of the infecting pathogen. Rapidly administered, targeted therapy is also important to reduce the risk of resistance development among pathogens. Current practice for pathogen identification in BSI consists of time-consuming (24-72 hours) blood cultures. To be able to provide fast and patient tailored treatment, identification of the pathogen should be available as soon as possible, as patients in septic shock with inappropriate treatment have significantly lower survival rates $[4,5]$.

Culture-independent identification techniques, such as molecular diagnostics, will shorten time to result. Pathogen levels in blood of BSI patients can be as low as 1-10 colony forming units (CFU) per $\mathrm{ml}$, therefore several millilitres of blood may be required to reach clinically relevant sensitivity. This poses a problem since the amount of human DNA and haemoglobin present in such samples inhibit the pathogen-specific PCR [6]. In order to reach similar sensitivities as blood cultures, where input is in the order of $10-20 \mathrm{ml}$ per blood culture set, pathogen DNA enrichment methods should precede the identification PCR.

Recently, several molecular diagnostic tests for whole blood became commercially available (SepsiTest (Molzym), MagicPlex Sepsis Real-Time Test (Seegene), VYOO (SIRS Lab), and SeptiFAST (Roche)) and were evaluated by several independent research groups [7-13]. However, none of the abovementioned tests combines pathogen DNA enrichment with fast identification, they provide either pathogen DNA enrichment or fast sensitive detection. Only the Molzym test enables pathogen DNA enrichment based on enzymatic removal of human DNA (MolYsis) using an input volume of 1 to $5 \mathrm{ml}$ whole blood [14, 15]. However, the method is labour-intensive and the use of enzymes may make this test less stable. We therefore tested and evaluated a novel non-enzymatic and more rapid pathogen DNA enrichment method for blood samples, designated Polaris.

The main goal of this study was to evaluate the Polaris method and to compare its performance to the MolYsis method and a method that does not entail removal of human DNA (Triton-TrisEDTA - EasyMAG) [16]. These methods were compared using whole blood samples spiked with frequently recovered BSI microorganisms Staphylococcus aureus, Pseudomonas aeruginosa and Candida albicans, representing Gram-positive and Gram-negative bacteria, and a fungus, respectively. 


\section{Materials and Methods}

\section{Ethics statement}

In The Netherlands, healthy blood donors have to sign an informed consent form when donating blood at the Sanquin institute. In this form, medical research purposes are mentioned. Research institutes can buy this blood, and donors are anonimised. Therefore, no additional informed consent was required

\section{Spiking experiments}

EDTA blood from healthy human volunteers was obtained from Sanquin (bloodbank, Eindhoven, The Netherlands). Staphylococcus aureus (ATCC 25923), Pseudomonas aeruginosa (ATCC 27853), and Candida albicans (ATCC 90028) were used for spiking. All microorganisms were cultured overnight $(\mathrm{O} / \mathrm{N})$ on blood agar plates (TSA plates with $5 \%$ Sheep Blood, Fischer scientific, Aalst, Belgium). Subsequently, the cells were grown to mid log phase in Brain Hearth Infusion broth ( $S$. aureus) or LB ( $P$. aeruginosa) to ensure having a majority of actively growing cells. $C$. albicans was in mid log phase after the O/N culturing step. Hereafter, a ten-fold serial dilution was made in PBS (Merck, Darmstadt, Germany) and before spiking a live/dead staining (Life Technologies, Gent, Belgium) was performed as described by the manufacturer, to determine the ratio between live and dead pathogens (criterium used $>90 \%$ living bacteria). This was performed to confirm that the majority of cells is intact since the MolYsis and Polaris method will not allow enrichment of damaged cells and free DNA. To determine the CFU per $\mathrm{ml}, 100 \mu \mathrm{l}$ from several dilutions were plated onto blood agar plates and cultured O/N. Blood was spiked with the different dilutions, yielding 0-1000 $\mathrm{CFU} / \mathrm{ml}$ blood. Reference samples consisted of similar amounts of pathogens taken from the PBS dilution series directly subjected to lysis and extraction. See Figure 1 for an overview of the experimental set-up.

\section{DNA isolation methods}

Pathogen DNA was isolated with three different methods. The Triton-Tris-EDTA (TTE) pretreatment procedure (input $200 \mu \mathrm{l}$ blood) followed by EasyMAG isolation (BioMérieux, Marcy L'Etoile, France) was performed as described by Peters et al. [16]. The MolYsis complete 5 kit was used for pathogen DNA isolation from 1 and $5 \mathrm{ml}$ spiked whole blood as described by the manufacturer (Molzym GmbH, Bremen, Germany).

Details of the Polaris technology (Biocartis, Mechelen, Belgium) are described elsewhere (patents WO2012168003 A1 and WO2011070507 A1). For Polaris (Figure 2), 1 or 5 ml blood was mixed with an equal volume of selective lysis buffer (SLB) for 3 minutes, to lyse blood cells and fragment the released human DNA and then 1 or $5 \mathrm{ml}$ neutralization buffer was added. The selective lysis is based on a mild detergent to degrade the human cell membranes but 


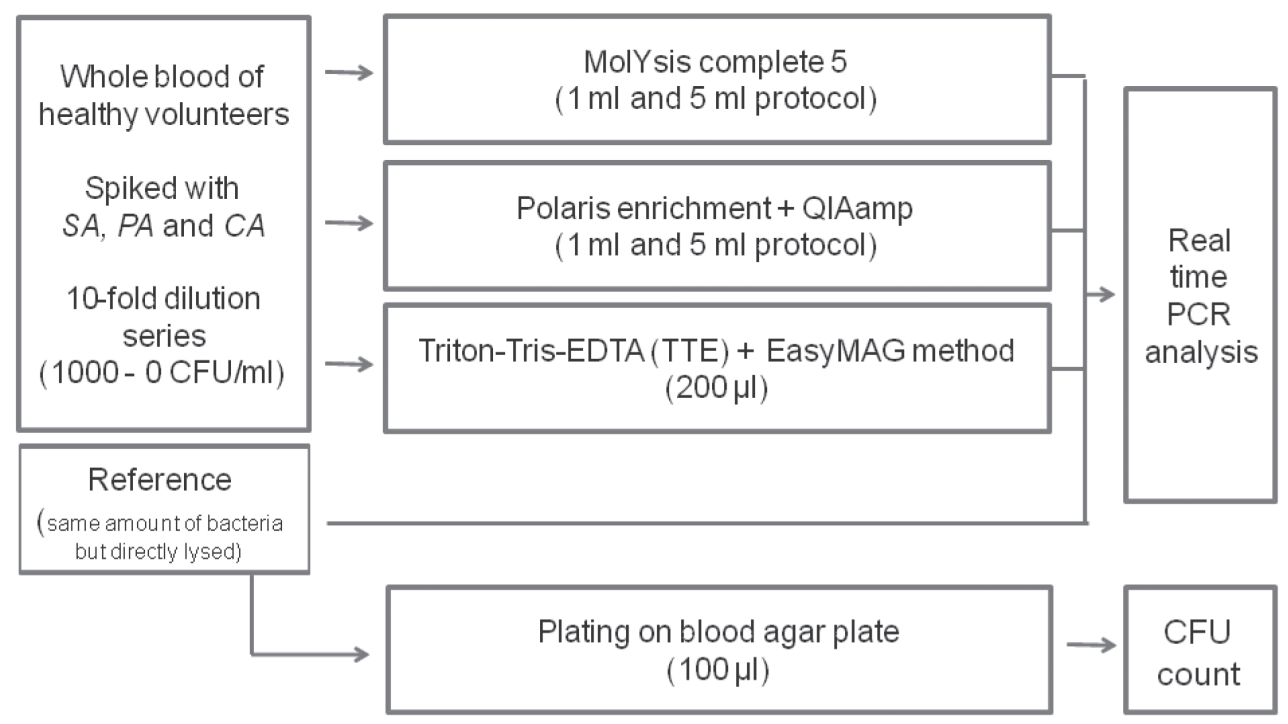

Figure 1. Flowchart of the experimental set-up.

SA, S. aureus; PA, P. aeruginosa; CA, C. albicans; CFU, colony forming unit.

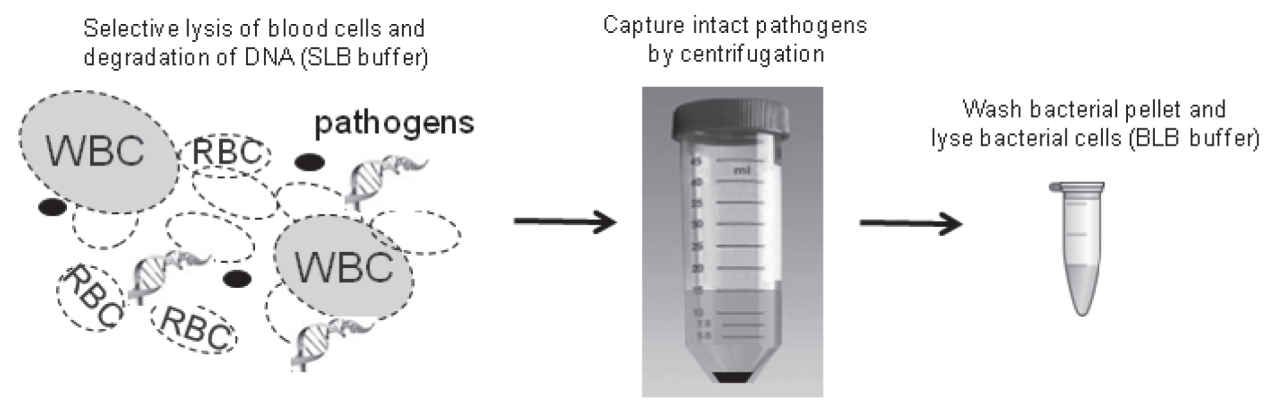

Figure 2. Overview of the Polaris method.

Whole blood is depicted, consisting of human cells and DNA, and some pathogens. In the first step, human cells and DNA are degraded and pathogens remain intact. In the second step, intact pathogens are pelleted by centrifugation. Finally, this pellet is washed and pathogens are lysed. Subsequently, DNA can be isolated (not depicted). WBC, white blood cell; RBC, red blood cell; SLB, selective lysis buffer; BLB, bug lysis buffer.

not the bacterial and fungal cell walls. An elevated $\mathrm{pH}$ will ensure degradation of the released nucleic acids. Therefore, this method focuses on the enrichment of the intact bacteria and fungi from blood and not potential free pathogen DNA. The selective lysis reaction needs to be controlled in time as Gram-negative bacteria might be lysed upon prolonged exposure. Therefore an equal volume of neutralization buffer is added after $3 \mathrm{~min}$. This buffer will ensure a complete arrest of the selective lysis treatment by lowering of the $\mathrm{pH}$ and dilution 
Table 1. Overview of primers and probes used for pathogen detection.

\begin{tabular}{|c|c|c|c|c|}
\hline Pathogen & gene & Forward primer & Reverse primer & Probe (FAM-BHQ1) \\
\hline$P$. aeruginosa & $\operatorname{reg} A$ & $\begin{array}{l}\text { TGCTGGTGGCACAG- } \\
\text { GACAT }\end{array}$ & $\begin{array}{l}\text { TTGTTGGTG- } \\
\text { CAGTTCCTCATTG }\end{array}$ & $\begin{array}{l}\text { CCAGATGCTTTGCCT- } \\
\text { CAACGTCG }\end{array}$ \\
\hline S. aureus & tuf & $\begin{array}{l}\text { TCCTGGTTCAATTA- } \\
\text { CACCACATACTG }\end{array}$ & $\begin{array}{l}\text { GGAAATAGAATTGTG- } \\
\text { GACGATAGTTTGA }\end{array}$ & $\begin{array}{l}\text { TGATAATACGTATACT- } \\
\text { TATGC }\end{array}$ \\
\hline C. albicans & ITS-2 & $\begin{array}{l}\text { GGAGGGCATGCCT- } \\
\text { GTTTG }\end{array}$ & $\begin{array}{l}\text { CAAGTCGTATTGCT- } \\
\text { CAACACCAA }\end{array}$ & $\begin{array}{l}\text { TCGTTTCTCCCT- } \\
\text { CAAACCGCTGGG }\end{array}$ \\
\hline
\end{tabular}

of the detergent to an ineffective concentration. At this moment in time, the pathogens will remain intact. Consecutively, suspensions were centrifuged for 15 minutes ( $5 \mathrm{ml}$ protocol) or 10 minutes ( $1 \mathrm{ml}$ protocol) at $2791 \mathrm{x}$ g. Pellets were resuspended in $1 \mathrm{ml}$ washing buffer and centrifuged for 10 minutes at maximum speed in a Eppendorf centrifuge. Resulting pellets were thoroughly resuspended in $200 \mu$ bacterial lysis buffer (BLB) and incubated for 10 minutes at $95^{\circ} \mathrm{C}$ on a thermomixer set at $1000 \mathrm{rpm}$. After addition of $20 \mu \mathrm{l}$ neutralization buffer 2, lysates were further processed for DNA purification using QIAamp blood mini kit columns (Qiagen, Venlo, The Netherlands) or the generic program of the EasyMAG device.

\section{Real-time PCR}

The RNAseP kit (Life technologies, Gent, Belgium) was used to measure the amount of human DNA. Species-specific real-time PCRs were performed to investigate the performance of each method. For detection of $S$. aureus the tuf gene based LightCycler 2.0 assay was used [17]. The primers and probes for detection of $S$. aureus, $P$. aeruginosa, and $C$. albicans are depicted in Table 1. PCR mix consisted of $12.5 \mu$ Taqman Universal fast $2 x$ mastermix (Applied Biosystems), $300 \mathrm{nM}$ primers, $200 \mathrm{nM}$ probe, and $10 \mu \mathrm{l}$ sample (1/10 of total eluate), water was added to an end volume of $25 \mu \mathrm{l}$. PCRs were performed on the Biorad CFX-96 under the following conditions; $3 \mathrm{~min} 95^{\circ} \mathrm{C}$ followed by 50 cycles of $15 \mathrm{sec}$ at $95^{\circ} \mathrm{C}$ and $1 \mathrm{~min}$ at $60^{\circ} \mathrm{C}$.

\section{Statistical analysis}

For analysis of the results the Fisher's exact test and one-way ANOVA were performed in SPSS (Version 19.0. Armonk, NY: IBM Corp). For one-way ANOVA analysis, the Bonferroni's correction for multiple comparisons was performed for comparison of the obtained Ct-values (RNAseP) for the different methods. For both statistical methods, a $p$-value less than 0.05 was considered significant. 


\section{Results}

\section{Performance of Polaris: effect of sample volume}

To test the effect of sample volume on sensitivity of the Polaris procedure, a range of pathogen concentrations was spiked in 1 and $5 \mathrm{ml}$ whole blood samples from healthy volunteers. Consistently lower cycle threshold (Ct) values were obtained in the PCRs when pathogen DNA enrichment was performed on $5 \mathrm{ml}$ instead of $1 \mathrm{ml}$ (Figure 3, grey bars (left side $1 \mathrm{ml}$, right side $5 \mathrm{ml}$ )). The difference in $\mathrm{Ct}$ value was less pronounced in the lower ranges of pathogen concentration (1 CFU/ml). However, at this concentration a higher detection rate (S. aureus $12.5 \%$ (1 $\mathrm{ml}$ ) versus 70\% (5 ml); $P$. aeruginosa 44\% (1 ml) versus 75\% (5 ml); C. albicans 75\% for both 1 and $5 \mathrm{ml}$ ) was observed for the $5 \mathrm{ml}$ samples compared to those derived from $1 \mathrm{ml}$, indicating that a $5 \mathrm{ml}$ sample provides a higher sensitivity than a $1 \mathrm{ml}$ sample. Furthermore, the Ct-values indicate that all tested pathogens were detected with similar efficiencies (Figure 4). The selective enrichment and the pathogen lysis step perform well for the different classes of pathogens, i.e. fungal, Gram-positive and Gram-negative bacterial organisms. At the same time, this demonstrates that no pathogens are lost during the selective lysis step.

Polaris-processed spiked blood samples were compared to reference samples, containing the same amount of pathogens, but then directly lysed in BLB. At all pathogen concentrations tested, the Polaris-processed samples yielded similar $\mathrm{Ct}$ values as the reference samples (Figure 4), demonstrating the absence of inhibition in the blood-derived samples. All nonspiked blood samples were negative in the PCRs.

\section{Effect of elution volume in DNA extraction}

Next, it was investigated which DNA purification method following Polaris enrichment would result in optimal detection, QIAamp (elution in $100 \mu$ I EB buffer as in protocol) or EasyMAG (elution in $25 \mu \mathrm{l}$ ), followed by PCR where in both cases $10 \mu \mathrm{l}$ eluate was used. To that end, 11 different $5 \mathrm{ml}$ blood samples each containing $1 \mathrm{CFU} / \mathrm{ml}$ of $S$. aureus were processed using Polaris. Five samples were purified using the QIAamp blood mini kit and six samples were processed on the EasyMAG. Using the EasyMAG generic protocol, 5 out of 6 samples were positive in the S. aureus PCR. With QIAamp only 3 out of 5 samples resulted in PCR signals. These preliminary results show that the combination of Polaris and EasyMAG makes it possible to put an equivalent of $10 / 25 \times 5 \mathrm{ml}=2 \mathrm{ml}$ blood in one PCR reaction and obtain an $5 / 6$ detection rate at a concentration of $1 \mathrm{CFU} / \mathrm{ml}$.

\section{Human DNA is efficiently removed}

To assess human DNA removal capacity of the different methods, an RNAseP PCR was performed. The obtained Ct-values (Figure 5) show that the TTE-EasyMAG method removed the least amount of human DNA (lowest Ct-value for RNAseP). One-way ANOVA analysis 

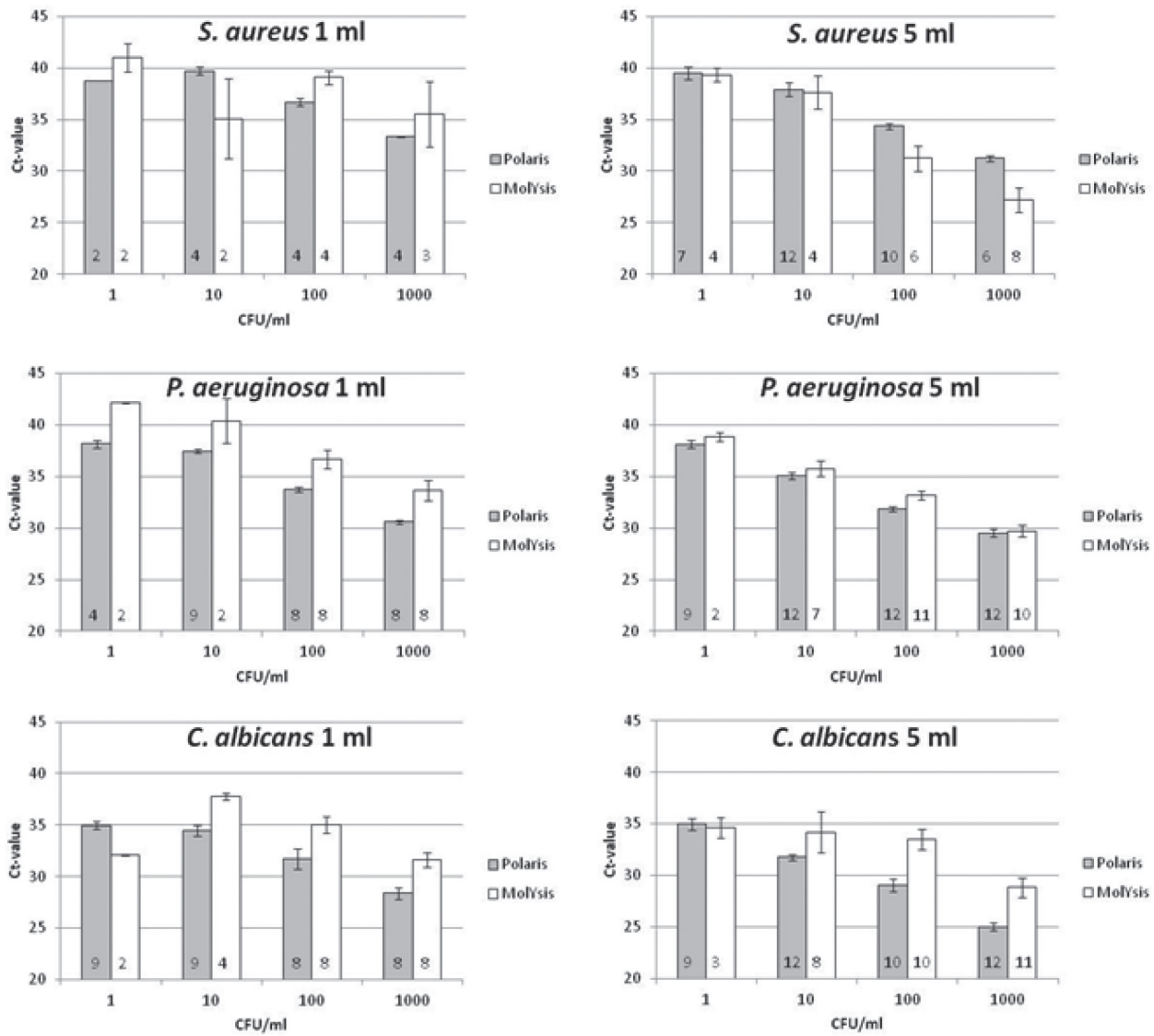

Figure 3. Comparison of Polaris and MolYsis methods using $1 \mathrm{ml}$ and $5 \mathrm{ml}$ spiked whole blood samples.

The grey bars represent the Polaris samples (1 or $5 \mathrm{ml}$ whole blood), and the white bars represent the MolYsis isolated samples ( 1 or $5 \mathrm{ml}$ whole blood). SEM is shown. The numbers in the bars represent the sample numbers. 

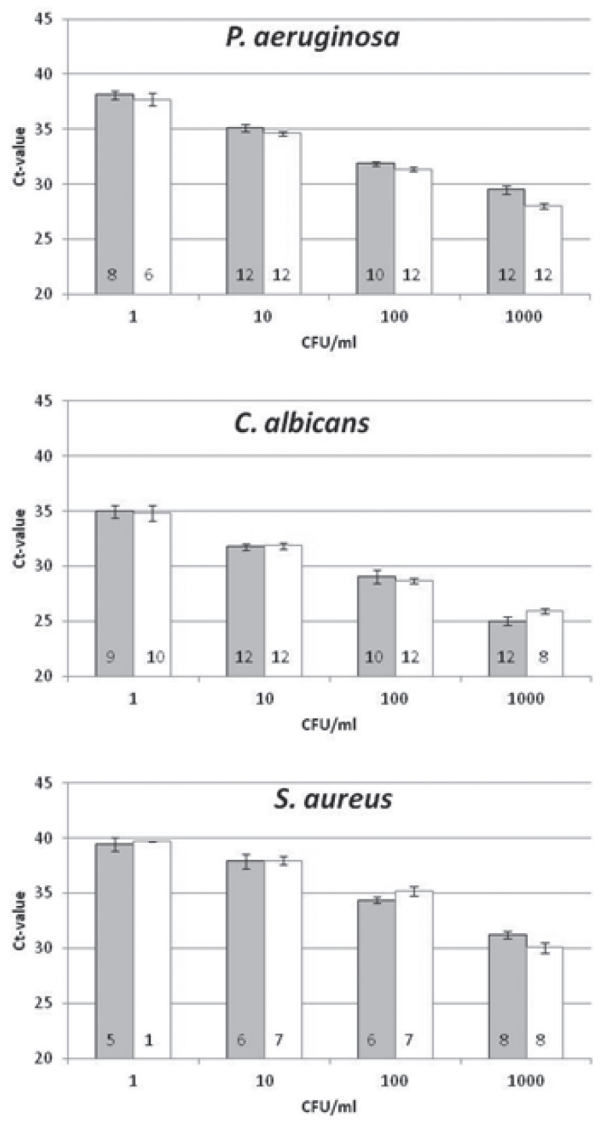

Figure 4. Polaris pathogen DNA isolation from reference (PBS) compared to $5 \mathrm{ml}$ whole blood. The indicated pathogens were spiked in $5 \mathrm{ml}$ whole blood or processed as reference samples as described in the Materials and Methods. For all pathogens similar $\mathrm{Ct}$ values were obtained when isolated from PBS or whole blood. The grey bars represent the spiked $5 \mathrm{ml}$ whole blood samples and the white bars the reference samples. SEM is shown. The numbers in the bars represent the sample numbers. 
indicated that this was statistically significant to all other methods ( $p<0.001)$, except when compared to MolYsis $1 \mathrm{ml}(p=0.156)$. The MolYsis method for $5 \mathrm{ml}$ of whole blood removes most human DNA as compared to all other methods $(p<0.000)$. Significant differences were also found when comparing Polaris for $1 \mathrm{ml}$ whole blood with both MolYsis for $1 \mathrm{ml}(p=0.002)$ and the MolYsis method for $5 \mathrm{ml}$ whole blood $(p<0.000)$, and when comparing MolYsis for $1 \mathrm{ml}$ with MolYsis for $5 \mathrm{ml}$ whole blood $(p<0.000)$. No significant difference in human DNA removal capacity was found between the Polaris methods for different volumes of whole blood $(p=0.548)$.

The lower $\mathrm{Ct}$ value for RNAseP in Figure 5 showed that the amount of residual human DNA was higher in the $1 \mathrm{ml}$ MolYsis protocol than in the $5 \mathrm{ml} \mathrm{MolYsis} \mathrm{protocol}$. For the $1 \mathrm{ml}$ protocol the volume of blood in the total lysate is $1 / 1.5$ or $66 \%$, whereas in the $5 \mathrm{ml}$ protocol this is $5 / 9$ or $55 \%$. In comparison, the $1 \mathrm{ml}$ Polaris protocol was more efficient in DNA removal than the $1 \mathrm{ml}$ MolYsis protocol, whereas for the $5 \mathrm{ml}$ protocols (MolYsis versus Polaris) it was the other way around. However, both the MolYsis and the Polaris method removed sufficient human background so no interference with the specific pathogen PCR was detected.

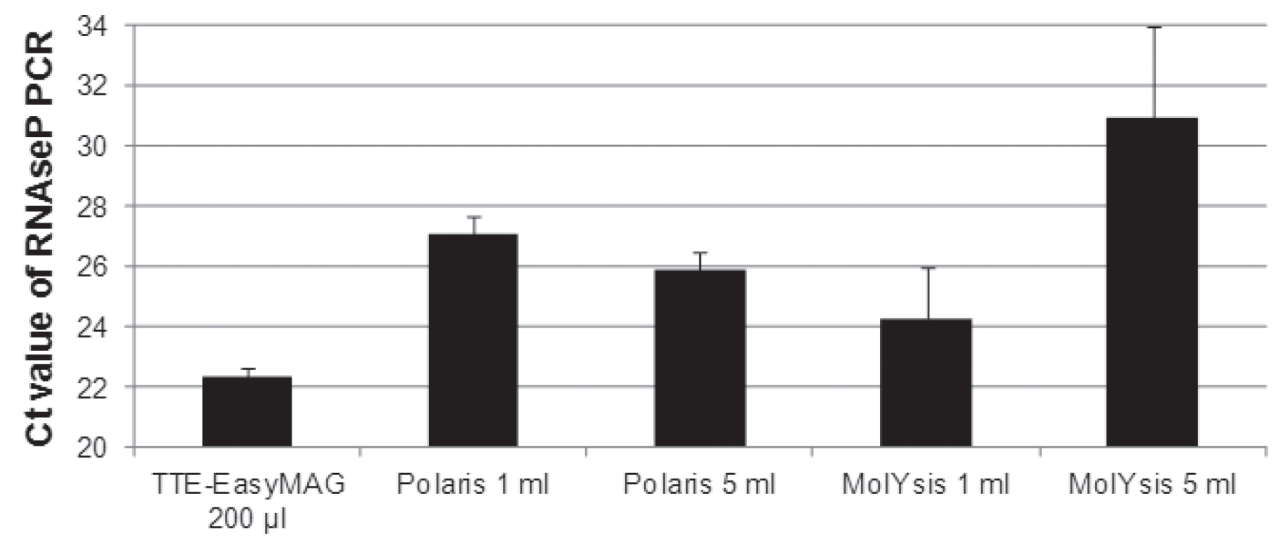

Figure 5. Human DNA removal by different procedures.

Ct value comparison of the RNAseP PCR for all pathogen DNA isolation procedures. RNAseP is a marker to measure human DNA removal after pathogen DNA isolation. Standard deviations are shown of at least 6 independent experiments. One-way ANOVA analysis indicated that TTE-EasyMAG removes the least amount of human DNA as compared to all other methods $(p<0.001)$, except when compared to MolYsis $1 \mathrm{ml}(p=0.156)$. MolYsis $5 \mathrm{ml}$ removes most human DNA as compared to all other methods $(p<0.000)$. Significant differences were also found when comparing Polaris $1 \mathrm{ml}$ with both MolYsis $1 \mathrm{ml}(p=0.002)$ and $5 \mathrm{ml}(p<0.000)$, and when comparing MolYsis $1 \mathrm{ml}$ with MolYsis 5 $\mathrm{ml}(p<0.000)$. 


\section{Comparison of Polaris, TTE-EasyMAG and MolYsis}

The TTE-EasyMAG procedure yielded higher Ct values for most samples compared to the Polaris samples (up to $6 \mathrm{Ct}$ difference). Polaris and MolYsis resulted in comparable Ct values for all pathogens (Figure 3). Both MolYsis and Polaris enabled detection of clinical relevant pathogen concentrations of 1-10 CFU/ml. In general, the variation in Ct values was much larger for MolYsis-processed samples than for samples processed with Polaris (Figure 3).

Calculations of detection rates, i.e. percentages of positive PCRs, demonstrated a detection rate of $100 \%$ for all pathogens at a concentration of $10 \mathrm{CFU} / \mathrm{ml}$ for the Polaris procedure (Table 2). The TTE-EasyMAG procedure performed much worse in this respect with a detection rate of only $58 \%, 60 \%$, and $79 \%$ for $10 \mathrm{CFU} / \mathrm{ml} \mathrm{S}$. aureus, C. albicans, and $P$. aeruginosa, respectively. MolYsis resulted in a detection rate of $50 \%, 67 \%$, and $58 \%$ for $10 \mathrm{CFU} / \mathrm{ml}$ S. aureus, C. albicans, and $P$. aeruginosa, respectively. Processing samples containing $1 \mathrm{CFU} / \mathrm{ml}$ never resulted in a $100 \%$ detection rate for the tested methods. The best results were obtained with Polaris as a $70 \%$ detection rate was obtained for S. aureus, and $75 \%$ for both $C$. albicans and $P$. aeruginosa. MolYsis detection rates at this pathogen concentration varied between 17 and 50\%, and TTE-EasyMAG between 20-36\%. All nonspiked blood samples were negative in the PCRs.

Table 2. Detection rates (percentage of positive PCRs) of 3 different DNA isolation methods in dilutions series of 1-1000 CFU/ml.

\begin{tabular}{|c|c|c|c|c|c|c|}
\hline & & \multicolumn{5}{|c|}{$\mathrm{CFU} / \mathrm{ml}$} \\
\hline & & 1000 & 100 & 10 & 1 & $p a, b, c$ \\
\hline \multirow{3}{*}{ 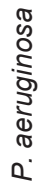 } & Polaris $5 \mathrm{ml}$ & $100 \%(12 / 12)$ & $100 \%(12 / 12)$ & $100 \%(12 / 12)$ & $75 \%(9 / 12)$ & $a(p=0.01)$ \\
\hline & MolYsis $5 \mathrm{ml}$ & $83 \%(10 / 12)$ & $92 \%(11 / 12)$ & $58 \%(7 / 12)$ & $17 \%(2 / 12)$ & $b(p=0.06)$ \\
\hline & TTE-EasyMAG & $100 \%(8 / 8)$ & $92 \%(11 / 12)$ & $79 \%(11 / 14)$ & $36 \%(5 / 14)$ & $c(p=0.39)$ \\
\hline \multirow{3}{*}{$\begin{array}{l}\mathscr{\infty} \\
\frac{d}{d} \\
\frac{\bigcup}{0} \\
\frac{0}{d} \\
ن\end{array}$} & Polaris $5 \mathrm{ml}$ & $100 \%(12 / 12)$ & $83 \%(10 / 12)$ & $100 \%(12 / 12)$ & $75 \%(9 / 12)$ & $a(p=0.04)$ \\
\hline & MolYsis $5 \mathrm{ml}$ & $92 \%(11 / 12)$ & $83 \%(10 / 12)$ & $67 \%(8 / 12)$ & $25 \%(3 / 12)$ & $b(p=0.08)$ \\
\hline & TTE-EasyMAG & $100 \%(10 / 10)$ & $100 \%(10 / 10)$ & $60 \%(6 / 10)$ & $30 \%(3 / 10)$ & $c(p=1.00)$ \\
\hline \multirow{3}{*}{ 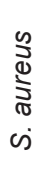 } & Polaris $5 \mathrm{ml}$ & $100 \%(6 / 6)$ & $100 \%(10 / 10)$ & $100 \%(12 / 12)$ & $70 \%(7 / 10)$ & $a(p=0.63)$ \\
\hline & MolYsis $5 \mathrm{ml}$ & $100 \%(8 / 8)$ & $75 \%(6 / 8)$ & $50 \%(4 / 8)$ & $50 \%(4 / 8)$ & $b(p=0.07)$ \\
\hline & TTE-EasyMAG & $100 \%(6 / 6)$ & $90 \%(9 / 10)$ & $58 \%(7 / 12)$ & $20 \%(2 / 10)$ & $c(p=0.32)$ \\
\hline
\end{tabular}

Fisher's exact test performed on $1 \mathrm{CFU} / \mathrm{ml}$ samples, statistically significant when $p \leq 0.05$. a; Polaris versus MolYsis, b; Polaris versus TTE-EasyMAG, c; MolYsis versus TTE-EasyMAG. 
The Fisher's exact test was performed to show significant differences between the obtained detection rates $(1 \mathrm{CFU} / \mathrm{ml})$ with the different pathogen DNA isolation methods for each pathogen. There was never a significant difference found, in detection rate, when comparing TTE-EasyMAG with MolYsis $(5 \mathrm{ml})$ or Polaris $(5 \mathrm{ml})$ for all tested pathogens. However, TTEEasyMAG compared with Polaris showed to have lower $p$-values ( $p$ between $0.06-0.08$ ) as when compared with MolYsis ( $p$ between $0.32-1.00$ ). For $P$. aeruginosa, Polaris had a significant better detection rate when compared to MolYsis $(p=0.01)$. This difference was also seen for $C$. albicans $(p=0.04)$. No significant difference in detection rate for $S$. aureus was found between Polaris and MolYsis $(p=0.63)$.

\section{Discussion}

In this study, different pathogen DNA isolation methods for whole blood were compared. We showed that both MolYsis and Polaris enrichment followed by DNA isolation and real-time PCR enabled reliable and sensitive detection of bacteria and fungi from $5 \mathrm{ml}$ blood. MolYsis and the TTE-EasyMAG procedure resulted in a lower number of positive PCRs (detection rate) as compared to Polaris, especially in the lower limit of detection (1-10 CFU/ml).

The detection rates for $P$. aeruginosa and $S$. aureus detection are similarly high at a pathogen concentration of $1 \mathrm{CFU} / \mathrm{ml}$ when using Polaris (9/12 vs. 7/10) (Table 2). In contrast, using MolYsis enrichment, the detection rate for $P$. aeruginosa is considerably lower than that for Gram-positive S. aureus detection (2/12 vs. 4/8). Possibly, Gram-negative bacteria which generally are considered to be more fragile than Gram-positives may be negatively affected by the chaotropic buffer used in the MolYsis protocol to lyse human cells [18]. Furthermore, the $\mathrm{Ct}$ values obtained in the $C$. albicans PCR are lower compared to $C t$ values obtained in the other PCRs. This might be the result of copy number variations ( 5 versus single copy) [19]. However, the detection limits of all PCRs are similar.

Human DNA, which can interfere in the PCR reaction, was not removed when the TTEEasyMAG procedure was used. In contrast, Polaris and MolYsis enrichment resulted in substantial removal of human DNA as was shown by the RNAseP results and the fact that the reference and whole blood samples showed similar $\mathrm{Ct}$ values. There are differences in the ratio of blood and lysis buffer volumes between the 1 and $5 \mathrm{ml} \mathrm{MolYsis} \mathrm{protocols.} \mathrm{It} \mathrm{was}$ noticed that when using MolYsis the $1 \mathrm{ml}$ blood lysates were much more viscous than the $5 \mathrm{ml}$ lysates. Apparently, DNAse treatment is much less efficient in the more viscous $1 \mathrm{ml}$ lysate. This might also explain the high variability in residual human DNA levels in the $1 \mathrm{ml}$ MolYsis protocol.

In general, Ct values obtained after Polaris processing were much more constant than those after MolYsis processing. Several steps in the MolYsis procedures may contribute to this 
variation. Next to the chaotropic buffer mentioned above, the use of an enzyme to degrade DNA may yield variable results, due to enzyme instability. Furthermore, bacterial lysis is based on a mix of lytic enzymes and proteinase K. The Polaris procedure does not use chaotropic agents nor enzymes, but only chemicals that should remain stable over time. Furthermore, it was demonstrated that Polaris pathogen enrichment can be combined with both QIAamp and EasyMAG (generic) DNA purification. The preliminary data showed that Polaris combined with EasyMAG DNA purification holds most promise to obtain reliable data at borderline concentrations of $1 \mathrm{CFU} / \mathrm{ml}$. The benefit of using more concentrated DNA as input (EasyMAG) in the PCR was not negatively affected by concurrent concentration of inhibitory substances.

Polaris and MolYsis have shown to be valuable in spiked blood samples since they can handle large blood volumes. Clinical evaluation of Polaris is presently ongoing in comparison to MolYsis, which is clinically validated. Preliminary results of this ongoing study (Emergency Care Unit, Jeroen Bosch Hospital) show that both pathogen enrichment procedures work for clinical samples. Residual blood was collected, left over from standard diagnostics, from patients with blood cultures positive for $S$. aureus (1 culture) or $S$. pneumoniae (2 cultures) (data not shown). This approach limited the volume of usable blood to $1 \mathrm{ml}$ for each method. Polaris was followed by DNA purification using EasyMAG. We were able to detect S. pneumoniae and $S$. aureus in all 3 samples with corresponding positive blood cultures, indicating promising potential for both the Polaris and MolYsis procedure in clinical use.

Several molecular sepsis diagnosis tests have become commercially available recently, i.e. Roche's SeptiFAST, Seegene's MagicPlex Sepsis Test, VYOO (SIRS lab) and Molzym's SepsiTest. It has been shown, by independent research groups, that these diagnostic tests are complementing conventional culture techniques [12, 13, 20, 21]. Especially in antibiotictreated patients, molecular diagnostics can provide identification under conditions where blood cultures remain negative. Recent publications by Kühn and Wellinghausen [11, 12] show the value of Molzym's SepsiTest. Both described that the initial analysis, indicating the absence or presence of pathogens, can be performed in approximately 4 hours. Subsequent sequencing needs to be performed for specific pathogen identification. This approach has the advantage that any pathogen will be identified, but it takes an additional 4 hours (in an optimal setting) to obtain that result. Still, pathogen identification is available within one working day, which is faster compared to conventional culture techniques that take at least 24-72 hours for pathogen identification. Assays like SeptiFAST and Seegene's MagicPlex Sepsis Test have other limitations. In the SeptiFAST procedure no enrichment of pathogen DNA is included, this limits the maximal useable input blood volume to $1.5 \mathrm{ml}$ with an equivalent of only $0.167 \mathrm{ml}$ blood present in the PCR reaction. The detection system used in the SeptiFAST method enables rapid identification of 25 pathogens by multiplex real-time PCR followed by melting curve analysis. Seegene's MagicPlex real-time PCR test can be used in combination 
with MolYsis pathogen enrichment. The real-time PCR test enables the detection of $90 \mathrm{BSI}$ causing pathogens, but only 27 pathogens can be identified to the species level. The main disadvantage of the Seegene system is the fact that one first needs to create an amplicon bank via conventional PCR. Next, the vial containing PCR amplicons needs to be opened for subsequent signal amplification in a real-time PCR instrument. Most routine diagnostic laboratories would not allow this setup, as contamination risks exist. Polaris enrichment can be combined with established sepsis tests to be able to ensure broad pathogen detection from clinical samples.

In conclusion, Polaris and MolYsis enrichment followed by DNA isolation and real-time PCR enables reliable and sensitive detection of bacteria and fungi from $5 \mathrm{ml}$ blood. However, Polaris is slightly more sensitive and faster providing pathogen identification within 3 hours. To further enable its clinical value, Polaris is currently being automated in a closed disposable cartridge to reduce the hands on time to 1-2 $\mathrm{min}$, to be faster, and less prone to contamination. Furthermore, the combination of the Polaris cartridge with commercially available sepsis tests is currently being evaluated in a prospective clinical study using $5 \mathrm{ml}$ whole blood.

\section{Acknowledgements}

We thank Lieke Wielders (M.Sc) and dr. Mirrian Hilbink for their valuable contribution to this manuscript. Part of this research was performed within the framework of CTMM, the Center for Translational Molecular Medicine (www.ctmm.nl), project MARS (grant 04I-201). Philips Research, and later, Biocartis kindly provided Polaris and MolYsis components for this study. 


\section{References}

1. van Gestel, A., et al., Prevalence and incidence of severe sepsis in Dutch intensive care units. Crit Care, 2004. 8(4): p. R153-62.

2. Vincent, J.L. and E. Abraham, The last 100 years of sepsis. Am J Respir Crit Care Med, 2006. 173(3): p. 256-63.

3. Wisplinghoff, H., et al., Nosocomial bloodstream infections in US hospitals: analysis of 24,179 cases from a prospective nationwide surveillance study. Clin Infect Dis, 2004. 39(3): p. 309-17.

4. Kumar, A., et al., Initiation of inappropriate antimicrobial therapy results in a fivefold reduction of survival in human septic shock. Chest, 2009. 136(5): p. 1237-48.

5. Valles, J., et al., Community-acquired bloodstream infection in critically ill adult patients: impact of shock and inappropriate antibiotic therapy on survival. Chest, 2003. 123(5): p. 1615-24.

6. Sachse, S., et al., Truncated human cytidylate-phosphate-deoxyguanylate-binding protein for improved nucleic acid amplification technique-based detection of bacterial species in human samples. J Clin Microbiol, 2009. 47(4): p. 1050-7.

7. Dierkes, C., et al., Clinical impact of a commercially available multiplex PCR system for rapid detection of pathogens in patients with presumed sepsis. BMC Infect Dis, 2009. 9: p. 126.

8. Lehmann, L.E., et al., A multiplex real-time PCR assay for rapid detection and differentiation of 25 bacterial and fungal pathogens from whole blood samples. Med Microbiol Immunol, 2008. 197(3): p. 313-24.

9. Tsalik, E.L., et al., Multiplex PCR to diagnose bloodstream infections in patients admitted from the emergency department with sepsis. J Clin Microbiol, 2010. 48(1): p. 26-33.

10. Wallet, F., et al., Preliminary clinical study using a multiplex real-time PCR test for the detection of bacterial and fungal DNA directly in blood. Clin Microbiol Infect, 2010. 16(6): p. 774-779.

11. Wellinghausen, N., et al., Diagnosis of bacteremia in whole-blood samples by use of a commercial universal 16S rRNA gene-based PCR and sequence analysis. J Clin Microbiol, 2009. 47(9): p. 2759-65.

12. Kuhn, C., et al., Evaluation of commercial universal rRNA gene PCR plus sequencing tests for identification of bacteria and fungi associated with infectious endocarditis. J Clin Microbiol, 2011. 49(8): p. 2919-23.

13. Fitting, C., et al., DNAemia Detection by Multiplex PCR and Biomarkers for Infection in Systemic Inflammatory Response Syndrome Patients. PLoS One, 2012. 7(6): p. e38916.

14. Hansen, W.L., C.A. Bruggeman, and P.F. Wolffs, Evaluation of new preanalysis sample treatment tools and DNA isolation protocols to improve bacterial pathogen detection in whole blood. J Clin Microbiol, 2009. 47(8): p. 2629-31.

15. Horz, H.P., et al., Selective isolation of bacterial DNA from human clinical specimens. J Microbiol Methods, 2008. 72(1): p. 98-102.

16. Peters, R.P., et al., Quantitative detection of Staphylococcus aureus and Enterococcus faecalis DNA in blood to diagnose bacteremia in patients in the intensive care unit. J Clin Microbiol, 2007. 45(11): p. 3641-6.

17. Loonen, A.J., et al., Acceleration of the direct identification of Staphylococcus aureus versus coagulase-negative staphylococci from blood culture material: a comparison of six bacterial DNA extraction methods. Eur J Clin Microbiol Infect Dis, 2011. 30: p. 337-342.

18. Schneegurt, M.A., S.Y. Dore, and C.F. Kulpa, Jr., Direct extraction of DNA from soils for studies in microbial ecology. Curr Issues Mol Biol, 2003. 5(1): p. 1-8.

19. Lan, J., et al., Direct detection and genotyping of Chlamydia trachomatis in cervical scrapes by using polymerase chain reaction and restriction fragment length polymorphism analysis. $\mathrm{J}$ Clin Microbiol, 1993. 31(5): p. 1060-5.

20. Yanagihara, K., et al., Evaluation of pathogen detection from clinical samples by real-time polymerase chain reaction using a sepsis pathogen DNA detection kit. Crit Care, 2010. 14(4): p. R159.

21. Bloos, F., et al., A multicenter trial to compare blood culture with polymerase chain reaction in severe human sepsis. Intensive Care Med, 2010. 36(2): p. 241-7. 



\section{Chapter 8}

Biomarkers and molecular analysis to improve bloodstream infection diagnostics in an emergency care unit

Anne J.M. Loonen 1,6 , Cornelis P.C. de Jager², Janna Tosserams², Ron Kusters ${ }^{3}$,

Mirrian Hilbink ${ }^{4}$, Peter C. Wever ${ }^{5}$, Adriaan J.C. van den Brule ${ }^{1,6}$

Jeroen Bosch Hospital, ${ }^{1}$ Laboratory of Molecular Diagnostics, ${ }^{2}$ Department of Intensive Care and Emergency Medicine, ${ }^{3}$ Department of Clinical Chemistry and Haematology, ${ }^{4}$ Jeroen Bosch Academy, ${ }^{5}$ Department of Medical

Microbiology and Infection Control, 's-Hertogenbosch, The Netherlands

${ }^{6}$ Fontys University of Applied Sciences, Department of Medical Molecular Diagnostics, Eindhoven, The Netherlands 


\section{Abstract}

Bloodstream infections (BSI) are associated with high morbidity and mortality. Fast and accurate identification of the etiologic pathogen is, therefore, of clinical importance. Molecular assays for pathogen detection from whole blood are currently available. However, their exact clinical value remains to be determined and these assays are still expensive. The use of biomarkers may assist the physician in preselecting patients for immediate molecular testing besides blood culture.

In this study, 140 patients with $\geq 2$ systemic inflammatory response syndrome criteria and clinical signs of infection presenting at the emergency department of our hospital were included. C-reactive protein (CRP), neutrophil-lymphocyte count ratio (NLCR), procalcitonin (PCT) and soluble urokinase plasminogen activator receptor (suPAR) levels were determined. One ml residual EDTA blood was obtained and selective pathogen DNA isolation was performed with MolYsis (Molzym). DNA samples were analysed for the presence of pathogens, using both the commercially available MagicPlex Sepsis Test (Seegene) and SepsiTest (Molzym), and results were compared to blood culture results.

Fifteen patients had to be excluded from the study, leaving 125 patients for further analysis. Of the 125 patient samples analysed, 27 presented with positive blood cultures of which 7 were considered to be contaminants. suPAR, PCT, and NLCR values were significantly higher in patients with positive blood cultures compared to patients without $(p<0.001)$. Receiver operating characteristic curves of the 4 biomarkers for differentiating bacteremia from nonbacteremia showed the highest area under the curve (AUC) for PCT ( 0.806 (95\% confidence interval 0.699-0.913)). When compared to blood cultures, the sensitivity, specificity, positive predictive value (PPV), and negative predictive value (NPV) for SepsiTest and MagicPlex Sepsis Test were $11 \%, 96 \%, 43 \%, 80 \%$, and $37 \%, 77 \%, 30 \%, 82 \%$, respectively.

In conclusion, both molecular assays perform poorly when one $\mathrm{ml}$ whole blood is used from emergency care unit patients. NLCR is a cheap, fast, easy to determine, and rapidly available biomarker, and therefore seems most promising in differentiating BSI from non-BSI patients for subsequent pathogen identification using molecular diagnostics. 


\section{Introduction}

Bloodstream infection (BSI) is a potential life-threatening condition that requires early diagnosis and rapid pathogen identification to initiate correct antibiotic or antifungal therapy [1-3]. BSI patients frequently display characteristic symptoms of Systemic Inflammatory Response Syndrome (SIRS) [4]. In general, blood culture sets are collected when $\geq 2$ SIRS symptoms are recognized and infection is suspected. Blood cultures are regarded as the "gold standard" for the detection of viable bacterial and fungal organisms from blood, but are time-consuming. Furthermore, the sensitivity of blood cultures decreases significantly when antibiotic therapy has been started before blood samples are taken $[5,6]$, or when fastidious or slow-growing pathogens need to be cultured.

Molecular assays may improve BSI diagnostics. Recently, several molecular assays became commercially available which can be used for pathogen detection from whole blood. SepsiTest (Molzym), a broad-range SYBR Green based real-time polymerase chain reaction (PCR) assay followed by sequencing, has been investigated in clinical studies and is considered a valuable tool in addition to blood cultures [7, 8]. Compared to blood cultures, the diagnostic sensitivity and specificity of the SepsiTest PCR were described to be 87.0 and $85.8 \%$, respectively [8]. MagicPlex Sepsis Test (Seegene) screens for 90 pathogens and 3 resistance markers (mecA, vanA, vanB). Subsequently, 27 pathogens can be identified to the species level. Recently, MagicPlex Sepsis Test has been investigated for rapid detection of invasive candidiasis in pediatric patients, and was shown to have a sensitivity and specificity of $50 \%$ and $94 \%$, respectively [9]. Currently no literature is available on the performance of MagicPlex Sepsis Test for detection of BSI in adults.

Several limitations of molecular assays currently exist. First, they require special pathogen DNA enrichment to detect the low number of pathogens present in whole blood samples. Second, technical expertise is required to perform the tests. Third, the clinical value of molecular assays remains to be elucidated. And finally, the DNA tests available are still expensive. These restrictions prevent molecular assays to become the next "gold standard" for diagnosis of BSI as it is difficult and costly to implement them in daily laboratory practise. Biomarkers can be used to preselect suspected BSI patients for additional DNA based assays.

Several biomarkers have been described as either being associated with the presence of BSI or suggested to have prognostic value for outcome of BSI. The most widely studied marker is C-reactive protein (CRP), which is an acute-phase protein released by the liver after the onset of inflammation. CRP is mostly used to assess the presence of infection and sepsis [10]. Procalcitonin (PCT) is the prohormone of calcitonin and was first reported as a marker of inflammation in 1993 [11]. Several studies have been published which investigated its clinical value in the diagnosis of bacterial infections, especially sepsis [12, 13]. Zahorec et 
al. were the first to propose to use the ratio of neutrophil and lymphocyte counts (neutrophil lymphocyte count ratio (NLCR)) as an additional marker of infection in clinical practice [14]. In patients with suspected community-acquired infection in an emergency care setting the NLCR proved to be a simple biomarker with discriminatory capacity in predicting bacteremia. Recently, it was shown that this marker can be used in the prediction of bacteremia in patients admitted to the emergency department [15].

The soluble form of the urokinase plasminogen activator receptor (suPAR) has gained growing interest because it is proposed as a predictor of disease severity and case fatality in patients with bacteremia [16]. suPAR plays a role in various immunological functions and is expressed on various cell types including neutrophils, lymphocytes, macrophages, endothelial cells and tumor cells [17]. The two biomarkers that have been most studied in patients with sepsis are CRP and PCT, both of which are described to be markedly elevated in patients with sepsis $[18,19]$.

In this study, we evaluated the ability of various biomarkers (CRP, PCT, suPAR, and NLCR) to predict $\mathrm{BSI}$ in patients with suspected community-acquired BSI upon admission to the emergency department (ED). Furthermore, the performances of two commercially available molecular assays were examined and compared to blood culture results.

\section{Materials and Methods}

\section{Ethics statement}

Individual patient consent was not obtained since all data used in this study were acquired retrospectively from the laboratory information system (LIS) without any additional blood sampling. The Internal Review Board of the Jeroen Bosch Hospital approved anonymous use of remnant whole blood, serum, and data retrieved from the LIS and waived the need for informed consent.

\section{Patients and microbiology}

In this retrospective study, 140 patients presenting at the ED with $\geq 2$ SIRS criteria as described by Bone et al. [4] were included during November-December 2011 and October-December 2012. Additional inclusion criteria were, (1) age above 18 years, (2) clinical suspicion of infection, (3) blood cultures ordered, (4) EDTA blood and serum drawn simultaneously with blood cultures, and (5) sufficient remnant EDTA blood and serum volume available for analysis. Fifteen patient samples were excluded from the study because of an alternative diagnosis without infection, leaving 125 patients for further analysis.

Blood cultures were drawn by the medical staff during the observation period in the ED. Routinely, two pairs of aerobic and anaerobic bottles were obtained and incubated for a 
maximum of 5 days (BacT/ALERT; bioMérieux, Marcy L'Etoile, France). All isolates from positive blood cultures were identified at the species level by using standard microbiological procedures including MALDI-TOF mass spectrometry (Bruker Daltonics $\mathrm{GmbH}$, Bremen, Germany). Positive blood cultures with Gram-positive skin bacteria, e.g. coagulase-negative staphylococci (CoNS) or infrequently isolated environmental bacteria were considered to be contaminants, and therefore excluded from statistical analysis.

After performing standard diagnostics, $1 \mathrm{ml}$ remnant EDTA blood (per patient) was frozen $\left(-80^{\circ} \mathrm{C}\right)$ in DNA free UMD tubes (Molzym, Bremen, Germany) until further processing. UMD tubes stabilize specimens, including whole blood, through avoidance of damage of pathogens by freeze-thaw effects. A minimum of $500 \mu \mathrm{l}$ serum was frozen $\left(-80^{\circ} \mathrm{C}\right)$ for retrospective determination of suPAR and procalcitonin levels.

\section{Biomarkers determination}

CRP levels were measured with the Dimension Vista 1500 (Siemens Healthcare diagnostics). WBC counts were determined on a Sysmex XE-2100 hematology analyzer (Sysmex Corporation, Kobe, Japan). Neutrophil-Lymphocyte Count Ratio (NLCR) was determined by dividing the absolute neutrophil count by the absolute lymphocyte count. To additionally determine suPAR and PCT levels, serum was thawed on ice. suPAR levels were determined by using the suPARnostic ELISA kit (Virogates, Copenhagen, Denmark) according to the manufacturer's instructions, and PCT levels were measured using the Cobas E411 (Roche Diagnostics).

\section{Molecular assays}

UMD tubes containing $1 \mathrm{ml}$ EDTA blood were thawed on ice. Subsequently, the UMD blood was used for pathogen DNA isolation with the semi-automated MolYsis method (Molzym, Bremen, Germany) as described by the manufacturer. Pathogen DNA was stored at $-20^{\circ} \mathrm{C}$ until further processing.

The obtained pathogen DNA was analysed with two molecular assays (1) SepsiTest (Molzym, Bremen, Germany), and (2) MagicPlex Sepsis Test (Seegene, Seoul, Korea). Both molecular assays were used as described in the manufacturers' manuals and can be used in combination with MolYsis pathogen DNA isolation.

SepsiTest is a broad-spectrum real-time PCR test using SYBR Green followed by sequencing of the positive samples. This test is able to detect more than 345 species within one working day (8 hours, including pathogen DNA isolation). Results were considered positive if sequencing was successful.

MagicPlex Sepsis Test is a real-time PCR test that screens for pathogens as well as for methicillin (mecA) and vancomycin (vanA and vanB) resistance at once. After creation of an amplicon bank via normal PCR, screening for more than 90 pathogens to the genus level and 
resistance markers is performed. Results are available within 5 hours (including pathogen DNA isolation). Subsequent selective identification of pathogens (only 27 pathogens can be identified to the species level) is possible within an additional 30 minutes. The 21 bacterial pathogens that can be identified to the species level are: Pseudomonas aeruginosa, Acinetobacter baumannii, Stenotrophomonas maltophilia, Serratia marcescens, Bacillus fragilis, Salmonella typhi, Klebsiella pneumoniae, Escherichia coli, Klebsiella oxytoca, Enterobacter cloacae, Proteus mirabilis, Enterobacter aerogenes, Staphylococcus aureus, Staphylococcus epidermidis, Staphylococcus haemolyticus, Streptococcus agalactiae, Streptococcus pyogenes, Streptococcus pneumoniae, Enterococcus faecalis, Enterococcus faecium, and Enterococcus gallinarum. In this study, the selective identification step for fungi was not performed (Candida albicans, Candida tropicalis, Candida parapsilosis, Candida glabrata, Candida krusei, and Aspergillus fumigatus).

\section{Statistical analyses}

Patients were divided in two groups, with or without positive blood cultures. The Chi-Square test was used to determine statistical differences based on age and gender distribution between the groups. With regard to the continuous variables, CRP, PCT, NLCR and suPAR, we firstly judged for fit to the normal distribution using stem-and-leaf plots and quantilequantile plots. As our data did not follow a normal distribution, Mann-Whitney $U$ tests were performed for comparison of variables in different groups. Receiver operating characteristics (ROC) curve analyses were performed for the single biomarkers and combinations in predicting blood culture positivity. ROC curves displayed sensitivity versus 1-specificity such that area under the curves (AUC) varied from 0.5-1.0, with higher values indicating increased discriminatory ability. A $p$-value of less than 0.05 was considered statistically significant. Statistical analyses were performed using SPSS (Version 19.0. Armonk, NY: IBM Corp).

\section{Results}

\section{Patients and microbiology}

Of the 125 patient samples analysed, 27 patients (21.6\%) presented with positive blood cultures (Table 1). In ten patients with positive blood cultures, E. coli was identified, in six patients Staphylococcus spp. including one S. aureus isolate, and in three patients Streptococcus spp. More Gram-negative bacteria were recovered (16/27, 59\%) as compared to Gram-positive bacteria (11/27, 41\%). All positive culture sets grew bacterial species, and no fungi were detected. Seven out of the 27 blood culture isolates were considered to contain contaminants of which five belonged to the group of CoNS. When excluding contaminants from the total results, 20/125 patients presented with clinically relevant positive blood cultures (16\%). 
Table 1. Microorganisms grown from positive blood cultures.

\begin{tabular}{ll}
\hline Gram-positive bacteria & Gram-negative bacteria \\
\hline Clostridium paraputrificum & *Brevundimonas diminuta \\
*Propionibacterium spp. & Enterobacter cloacae \\
Staphylococcus aureus & Escherichia coli (10) \\
*Staphylococcus capitis (2) & Klebsiella pneumoniae \\
*Staphylococcus epidermidis & Proteus mirabilis \\
*Staphylococcus hominis (2) & Proteus vulgaris \\
Streptococcus gallolyticus & Salmonella group C \\
Streptococcus pneumoniae & \\
Viridans streptococci & \\
$\mathbf{1 1}$ (41\%) & $\mathbf{1 6}$ (59\%) \\
\hline
\end{tabular}

${ }^{*}$ Considered as contaminant. Number between () indicates number of cultures positive with this pathogen.

Table 2. Data of study population and results for C-reactive protein (CRP), procalcitonin (PCT), soluble urokinase plasminogen activator receptor (suPAR), and neutrophil-lymphocyte count ratio (NLCR).

\begin{tabular}{lllll}
\hline & $\begin{array}{l}\text { Positive Blood } \\
\text { Culture }\end{array}$ & $\begin{array}{l}\text { Negative Blood } \\
\text { Culture }\end{array}$ & p-value & AUC \\
\hline Age (years) mean \pm SD & $68.9( \pm 17.3)$ & $60.4( \pm 18.0)$ & 0.018 & \\
Gender & 17 Male / 10 Female & $\begin{array}{l}57 \text { Male / 41 Female } \\
0.653\end{array}$ & \\
CRP mean $( \pm \mathrm{SD})(\mathrm{mg} / \mathrm{L})$ & $105( \pm 105)$ & $119( \pm 110)$ & 0.886 & 0.485 \\
Median (range) & $88(8-371)$ & $93(1-490)$ & & \\
PCT mean $( \pm \mathrm{SD})(\mathrm{ng} / \mathrm{mL})$ & $11.1( \pm 25.2)$ & $2.0( \pm 10.3)$ & $<0.001$ & 0.806 \\
Median (range) & $1.0(0.039-100)$ & $0.2(0.02-100)$ & & \\
suPAR mean $( \pm \mathrm{SD})(\mathrm{ng} / \mathrm{mL})$ & $10.0( \pm 6.2)$ & $6.2( \pm 3.4)$ & $<0.001$ & 0.793 \\
Median (range) & $8.7(2.9-26.1)$ & $5.5(2.1-29.6)$ & & \\
NLCR mean $( \pm \mathrm{SD})$ & $23.0( \pm 15.0)$ & $12.2( \pm 9.1)$ & $<0.001$ & 0.770 \\
Median (range) & $18.4(7.1-56.5)$ & $9.9(0.89-44.1)$ & & \\
\hline
\end{tabular}

Median and range, mean and standard deviation (SD) are displayed. $p$-value less than 0.05 is statistically significant (Chi-Square and Mann-Whitney $U$ test), area under curve (AUC) is linked to Figure 1. 
Relevant demographic data are depicted in Table 2. No significant difference was found when comparing gender distribution in both groups (with or without positive blood culture). However, patients with a positive blood culture were significantly older (68.9 \pm 17.3 years; mean $\pm \mathrm{SD})$ as compared to patients with a negative blood culture (60.4 \pm 18.0 years) $(p=0.018)$.

\section{Biomarkers}

Mean CRP levels between both groups (positive and negative blood culture) were similar (105 \pm 105 versus $119 \pm 110 \mathrm{mg} / \mathrm{L}, p=0.886$ ) (Table 2 ). PCT levels were significantly different $(p<0.001)$ between patients with and without positive blood cultures $(11.1 \pm 25.2$ versus 2.0 $\pm 10.3 \mathrm{ng} / \mathrm{mL}$ ). The NLCR was also significantly different in both groups, showing a mean of $23.0 \pm 15.0$ in patients with positive blood cultures. In the group with negative cultures a mean of $12.2 \pm 9.1$ was found ( $p<0.001$ ). A mean suPAR level of $10.0 \pm 6.2 \mathrm{ng} / \mathrm{mL}$ was found in the patient group having blood culture proven BSI as compared to a level of $6.2 \pm 3.4 \mathrm{ng} /$ $\mathrm{mL}$ in the patient group with negative blood cultures $(p<0.001)$.

In our study population, 4 patients died as a result of sepsis complications. Blood culture results, NLCR, CRP, PCT, and suPAR levels in these patients were as follows: patient I) K. pneumoniae, 48.0, $228.0 \mathrm{mg} / \mathrm{L}, 100.0 \mathrm{ng} / \mathrm{mL}, 21.6 \mathrm{ng} / \mathrm{mL}$, patient II) S. epidermidis (considered contaminant), 8.9, $165.0 \mathrm{mg} / \mathrm{L}, 1.04 \mathrm{ng} / \mathrm{mL}, 4.0 \mathrm{ng} / \mathrm{mL}$, patient III) S. gallolyticus, 22.3, $76.0 \mathrm{mg} / \mathrm{L}, 0.255 \mathrm{ng} / \mathrm{mL}, 16 \mathrm{ng} / \mathrm{mL}$, and patient IV) negative blood culture, 38.3, 42.0 $\mathrm{mg} / \mathrm{L}, 0.065 \mathrm{ng} / \mathrm{mL}, 37.0 \mathrm{ng} / \mathrm{mL}$.

ROC curve analysis showed that PCT had the highest area under the curve (AUC) for differentiating patients with blood culture proven BSI from patients without: 0.806 (95\% confidence interval $(\mathrm{Cl}) 0.699-0.913)$ (Figure 1). AUC values for the other biomarkers were as follows: suPAR 0.793 (95\% Cl 0.660-0.926), NLCR 0.770 (95\% Cl 0.662-0.879), and CRP 0.485 (95\% Cl 0.344-0.626). Regarding combinations of biomarkers, combining NLCR and suPAR was most promising and resulted in an AUC of 0.815 .

Sensitivity, specificity, positive predictive value (PPV) and negative predictive value (NPV) for predicting blood culture proven BSI for PCT, NLCR and suPAR and combinations are depicted in Table 3. A 100\% sensitivity and NPV were obtained when combining NLCR cutoff $\geq 10$ with suPAR cut-off $\geq 6.2 \mathrm{ng} / \mathrm{mL}$. However, specificity and PPV were low, i.e. $27 \%$ and $22 \%$, respectively. By using only NLCR with a cut-off $\geq 10$, sensitivity, specificity, PPV, and NPV were $85 \%, 51 \%, 26 \%$, and $94 \%$, respectively.

\section{Molecular assays}

In Table 4 the results of the molecular assays are depicted and compared to the blood culture results. When using SepsiTest, only three EDTA blood samples were found positive, S. gallolyticus, S. pneumoniae, and K. pneumoniae were correctly identified as found in 


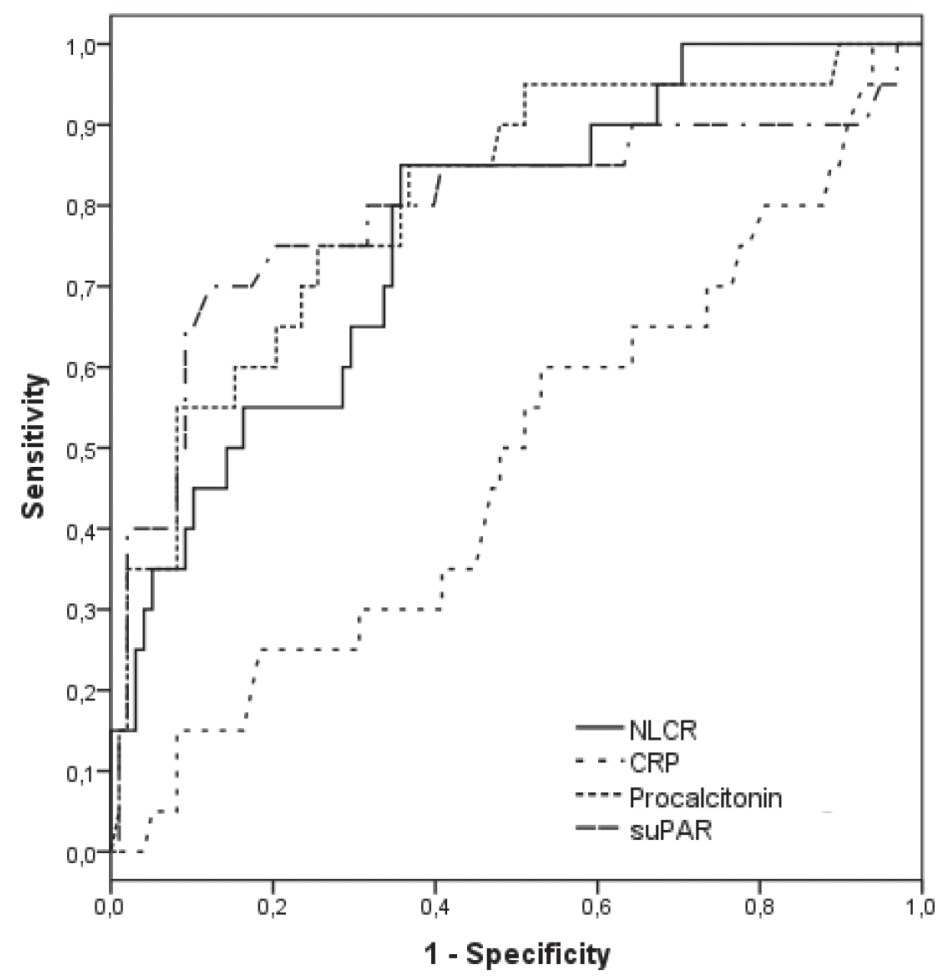

Figure 1. Receiver operating characteristic curves of four biomarkers for differentiating bacteremia from non-bacteremia.

C-reactive protein (CRP), neutrophil-lymphocyte count ratio (NLCR), procalcitonin and soluble urokinase plasminogen activator receptor (suPAR) are compared in respect to prediction of positive blood culture.

blood cultures. In contrast, MagicPlex Sepsis Test resulted in 12 positive PCR samples out of 27 blood culture positives. Detection of $S$. aureus, E. coli (6/10), E. cloacae, and P. mirabilis by MagicPlex Sepsis Test corresponded with blood culture results. The blood sample of one patient with a $S$. capitis blood culture isolate was only positive for mecA gene by MagicPlex Sepsis Test but not for Staphylococcus spp. MagicPlex Sepsis Test detected K. pneumoniae in the blood sample of one patient with a $S$. hominis blood culture isolate, while $S$. epidermidis was identified in blood from a patient with S. gallolyticus BSI.

SepsiTest resulted in four additional PCR positives blood samples from blood culture negative patients, i.e. Corynebacterium tuberculostearicum, Shigella sonnei/flexneri, Malassezia spp., and Cryptococcus spp. MagicPlex Sepsis Test resulted in an additional 23 PCR positive blood 
Table 3. Comparison of performance characteristics of the biomarkers and combinations in predicting bacteremia using different cut-off values.

\begin{tabular}{lllll}
\hline & Sensitivity & Specificity & PPV & NPV \\
\hline $\mathrm{NLCR} \geq 10$ & 85 & 51 & 26 & 94 \\
$\mathrm{NLCR} \geq 12$ & 70 & 65 & 29 & 91 \\
suPAR $\geq 6.2 \mathrm{ng} / \mathrm{mL}$ & 85 & 58 & 29 & 95 \\
suPAR $\geq 7.5 \mathrm{ng} / \mathrm{mL}$ & 80 & 77 & 42 & 95 \\
$\mathrm{PCT} \geq 2$ & 55 & 86 & 44 & 90 \\
$\mathrm{NLCR} \geq 10$ and/or suPAR $\geq 6.2 \mathrm{ng} / \mathrm{mL}$ & 100 & 27 & 22 & 100 \\
$\mathrm{NLCR} \geq 10$ and/or PCT $\geq 2 \mathrm{ng} / \mathrm{mL}$ & 95 & 49 & 28 & 98 \\
$\mathrm{NLCR} \geq 12$ and/or suPAR $\geq 7.5 \mathrm{ng} / \mathrm{mL}$ & 90 & 48 & 26 & 96 \\
$\mathrm{NLCR} \geq 12$ and/or PCT $\geq 2 \mathrm{ng} / \mathrm{mL}$ & 90 & 61 & 32 & 97 \\
\hline
\end{tabular}

Abbreviations, PPV: positive predictive value, NPV: negative predictive value, NLCR: neutrophillymphocyte count ratio, suPAR: soluble urokinase plasminogen activator receptor, PCT: procalcitonin.

Table 4. Overview of the results obtained with the molecular assays (SepsiTest and MagicPlex Sepsis Test) in comparison to blood culture results.

\begin{tabular}{lll}
\hline Blood Cultures & MolYsis + SepsiTest & MolYsis + MagicPlex Sepsis Test \\
\hline Positive Culture (27) & Positive PCR/Sequencing (3) & Positive PCR (12) \\
\hline Streptococcus gallolyticus & S. gallolyticus & Staphylococcus epidermidis \\
Streptococcus pneumoniae & S. pneumoniae & Negative \\
Viridans Streptococcus spp. & Negative & Negative \\
Staphylococcus aureus & Negative & S. aureus \\
Clostridium paraputrificum & Negative & Negative (not in kit) \\
Escherichia coli (10) & Negative (10) & E. coli (5), mix S. aureus/E. coli (1), \\
& & negative (4) \\
Enterobacter cloacae & Negative & E. cloacae \\
Klebsiella pneumoniae & K. pneumoniae & Negative \\
Proteus mirabilis & Negative & P. mirabilis \\
Proteus vulgaris & Negative & Negative \\
Salmonella spp. & Negative & Negative \\
Contaminants (7) & Negative (7) & Negative (5), K. pneumoniae \\
& & (culture Staphylococcus hominis), \\
& & mecA (culture Staphylococcus \\
Additional PCR positives & 4 & capitis) \\
\hline
\end{tabular}


samples from culture negative patients: $S$. epidermidis (13), other CoNS (not in identification kit) (2), S. pneumoniae (2), fungi (2), P. aeruginosa (2), A. baumannii (1), and K. oxytoca (1). In this study, the sensitivity, specificity, PPV, and NPV for SepsiTest and MagicPlex Sepsis Test (using blood culture as "gold standard") were 11\%, 96\%, 43\%, $80 \%$, and $37 \%, 77 \%$, $30 \%, 82 \%$, respectively. Negative and positive controls, included in each assay, showed adequate results indicating that each assay was performed correctly.

\section{Discussion}

Early identification of the pathogen causing BSI is essential for its adequate treatment, and it has been shown that when this treatment is initiated rapidly this will decrease BSI related mortality [20, 21]. Several tools are available which can be used and combined for optimal patient care: blood cultures, serum biomarkers, and potentially molecular assays for whole blood analysis.

Here, we studied the potential discriminating power of several biomarkers in the prediction of $\mathrm{BSI}$ in patients with SIRS and community-acquired infections presenting at the emergency department. In addition the performance of molecular assays was compared to the diagnostic yield of standard blood cultures. PCT, NLCR and suPAR are able to differentiate SIRS patients with and without blood culture proven bacteremia. The additional value of molecular assays in predicting BSI was low.

In this study, all patients fulfilled $\geq 2$ SIRS criteria and showed signs of infection, while only $16 \%$ presented with clinically relevant positive blood cultures (20/125). When including the blood culture contaminants, $22 \%$ of the patients had positive blood cultures (27/125). Other studies describe similar numbers. In a study analysing 104 patients of a surgical intensive care unit (ICU), Lodes et al. found that $20 \%$ of the drawn blood culture sets became positive [22]. Fitting et al. found $23 \%$ positive blood cultures in their ICU patient population [23]. However, Hoenigl et al. reported a positivity rate of $41 \%$ in a patient population from the emergency department [24]. Hoenigl et al. and Lodes et al. both used the BACTEC blood culture system as compared to the BacT/ALERT system used in this study. It has been described that BACTEC media has faster time to detection and increased bacterial recovery over the BacT/ALERT media [25]. Besides differences in culture systems, only two blood culture sets were drawn which were incubated for five days (this study), while Hoenigl et al. collected three pairs of blood cultures per patient and incubated for a maximum of seven days [24]. It remains difficult, however, to directly compare these study results as patient characteristics including disease severity might also differ besides culture methods.

PCT has been described to strongly correlate with the extent and severity of bacterial infections [26]. PCT is most frequently used in the management of infection and sepsis. 
Mencacci et al. have investigated if PCT serum levels could predict a positive PCR result (SeptiFAST, Roche) [27]. They found that PCT (cut-off value $\geq 0.37 \mathrm{ng} / \mathrm{ml}$ ) could be used in an unselected population of patients with fever and suspected sepsis to predict SeptiFAST PCR results. We have shown that PCT, as compared to the other biomarkers in this study, has the highest specificity in predicting bacteremia in SIRS patients. However, as PCT is an expensive biomarker, pre-screening of SIRS patients for further DNA analysis using PCT might be less cost-effective.

The use of suPAR levels at the emergency department has mostly been described in relation to categorizing patients according to their disease severity [28]. In this study, significant differences were found for suPAR levels between SIRS patients with and without positive blood cultures. However, only $2 / 4$ patients who died had high suPAR levels $(>16 \mathrm{ng} / \mathrm{mL}$ ). The suPAR levels of these patients were found between $4.4-26 \mathrm{ng} / \mathrm{mL}$. More studies, investigating suPAR levels in patients from the emergency department, need to be performed to clarify the usefulness of this relatively new biomarker.

De Jager et al. have described the NLCR in relation to predicting bacteremia at the emergency department [15]. NLCR (cut-off $\geq 10$ ) was shown to have a higher prognostic accuracy as compared to other biomarkers. In this study, prediction of community-acquired bacteremia using a NLCR cut-off $\geq 10$ resulted in a sensitivity, specificity, PPV, and NPV of $85 \%, 51 \%$, $26 \%$, and $94 \%$, respectively. Based on NLCR (cut-off $\geq 10$ ), 65/118 patients in our study would have been selected for molecular analysis. A higher cut-off value, i.e. $\geq 12$, results in decreased sensitivity but increased specificity. However, it is important to avoid SIRS patients to be falsely considered as negative BSI cases. For pre-screening purposes high sensitivity is preferred.

In this study, we investigated the performances of two molecular assays as compared to blood cultures. The sensitivities and specificities for SepsiTest were $11 \%$ and $96 \%$ compared to $37 \%$ and $77 \%$ for MagicPlex Sepsis Test. In this study, MagicPlex Sepsis Test and blood cultures showed similar results in $72 \%$ of the samples (both positive and negative samples). No reports were found describing the performance MagicPlex Sepsis Test in adult SIRS patients. Therefore, we can only compare the obtained SepsiTest results to other published studies. Kuhn et al. described a sensitivity of $85 \%$ for SepsiTest in patients with endocarditis [7]. Wellinghausen et al. describe a concordance of $86 \%$ for SepsiTest PCR and blood cultures in ICU patients with SIRS or sepsis [8]. Although, in this study, a concordance of $82 \%$ was found between SepsiTest and blood cultures for patients presenting with SIRS symptoms at the emergency department, the sensitivity compared to the blood culture gold standard was only $11 \%$. Different patient populations were used (ICU versus emergency department), and this might explain the difference in patient-related sensitivity $(85 \%$ and $82.4 \%$ versus $11 \%$ in this study). The performance of molecular assays is mostly investigated in ICU patient populations [8, 23, 29]. In general, more critically ill patients are hospitalized 
at the ICU as compared to the ED. We speculate that the ICU patient population might suffer from higher bacterial loads which can be more easily detected from one $\mathrm{ml}$ whole blood. It would be interesting to study bacterial loads in different patient populations.

Four patients from our study population died, and 3 of those patients had positive blood cultures (Klebsiella pneumoniae, Streptococcus gallolyticus and Staphylococcus epidermidis). SepsiTest correctly identified K. pneumoniae and S. gallolyticus, whereas MagicPlex Sepsis Test did not identify these pathogens from whole blood. As these patients died, this might indicate that these patients were more critically ill and suffered from higher bacterial loads, as has been described by Peters et al. [30]. It is unknown why MagicPlex Sepsis Test did not show positive PCR signals in the whole blood samples of these specific patients.

SepsiTest resulted in four PCR positive samples from blood culture negative patients. Three out of these four can be considered contaminants as these can be found on skin (Corynebacterium tuberculostearicum, Malassezia spp., and Cryptococcus spp). However, Shigella sonnei/flexneri is a clinically significant pathogen that was not detected with blood culture. MagicPlex Sepsis Test resulted in an additional 23 PCR positive samples in blood culture negative patients. CoNS species (15) can be considered contaminants, however, $S$. pneumoniae (2), fungi (2), P. aeruginosa (2), A. baumannii (1), and K. oxytoca (1) are clinical relevant pathogens. Prospective studies are needed to investigate the clinical value of additional positive samples using molecular diagnostics.

Molecular-based technologies are emerging as promising tools, in addition to blood cultures, for rapid identification of the etiological agents of BSI [31, 32]. However, several limitations exist (i.e. costs and the need for special equipment) which negatively affect the implementation of these techniques for routine laboratory diagnostics [33]. A limitation of this study is the use of only one $\mathrm{ml}$ residual whole blood for molecular analysis. It has been shown that detection rates obtained using molecular assays are higher when $5 \mathrm{ml}$ whole blood is used as compared to only one $\mathrm{ml}$ [34]. However, in this study residual whole blood, which was left after performing standard diagnostic tests, was used and only one $\mathrm{ml}$ whole blood could be processed and not $5 \mathrm{ml}$. More detailed investigation is necessary to select the best molecular assay available today. Furthermore, only prospective studies should be performed, using at least $5 \mathrm{ml}$ (or more) whole blood from suspected BSI patients. This should allow more optimal detection of pathogens from whole blood. Besides that, cost effectiveness analysis is needed to study the effect of implementation of a molecular assay on patient samples preselected, for instance based on NLCR results, in addition to blood cultures.

In summary, of all the biomarkers studied, PCT, suPAR and NLCR are suitable to differentiate SIRS patients with and without positive blood cultures. NLCR is a rapidly available, cheap, and easy to determine biomarker. Therefore, NLCR is a promising biomarker to preselect suspected BSI patients for molecular analysis besides blood culture. Unfortunately, the molecular assays available to date are not yet suitable for analysis of one $\mathrm{ml}$ remnant whole 
blood samples from patients at the emergency care unit. In addition, the clinical significance of DNA positivity in blood samples which remain culture negative needs further investigation.

\section{Acknowledgements}

We want to thank Ing. Eric Samuels for determining procalcitonin levels in the serum samples. We thank Biocartis for providing MagicPlex Sepsis Test (Seegene), Molzym (Bremen, Germany) for providing discount on MolYsis SepsiTest, and suPARnostic (Kopenhagen, Denmark) for providing discount on the used suPAR kits. No other funding was obtained. 


\section{References}

1. Emonet, S. and J. Schrenzel, How could rapid bacterial identification improve the management of septic patients? Expert Rev Anti Infect Ther, 2011. 9(9): p. 707-9.

2. Kumar, A., et al., Duration of hypotension before initiation of effective antimicrobial therapy is the critical determinant of survival in human septic shock. Crit Care Med, 2006. 34(6): p. 158996.

3. Vincent, J.L., Clinical sepsis and septic shock--definition, diagnosis and management principles. Langenbecks Arch Surg, 2008. 393(6): p. 817-24.

4. Bone, R.C., et al., Definitions for sepsis and organ failure and guidelines for the use of innovative therapies in sepsis. The ACCP/SCCM Consensus Conference Committee. American College of Chest Physicians/Society of Critical Care Medicine. Chest, 1992. 101(6): p. 1644-55.

5. Grace, C.J., et al., Usefulness of blood culture for hospitalized patients who are receiving antibiotic therapy. Clin Infect Dis, 2001. 32(11): p. 1651-5.

6. McKenzie, R. and L.G. Reimer, Effect of antimicrobials on blood cultures in endocarditis. Diagn Microbiol Infect Dis, 1987. 8(3): p. 165-72.

7. Kuhn, C., et al., Evaluation of commercial universal rRNA gene PCR plus sequencing tests for identification of bacteria and fungi associated with infectious endocarditis. J Clin Microbiol, 2011. 49(8): p. 2919-23.

8. Wellinghausen, N., et al., Diagnosis of bacteremia in whole-blood samples by use of a commercial universal 16S rRNA gene-based PCR and sequence analysis. J Clin Microbiol, 2009. 47(9): p. 2759-65.

9. Serra, J., et al., Clinical evaluation of the Magicplex Sepsis Real-time Test (Seegene) to detect Candida DNA in pediatric patients. Crit Care, 2012. 16: p. 21.

10. Uzzan, B., et al., Procalcitonin as a diagnostic test for sepsis in critically ill adults and after surgery or trauma: a systematic review and meta-analysis. Crit Care Med, 2006. 34(7): p. 1996-2003.

11. Assicot, M., et al., High serum procalcitonin concentrations in patients with sepsis and infection. Lancet, 1993. 341(8844): p. 515-8.

12. Giamarellos-Bourboulis, E.J., et al., Assessment of procalcitonin as a diagnostic marker of underlying infection in patients with febrile neutropenia. Clin Infect Dis, 2001. 32(12): p. 171825.

13. Petrikkos, G.L., et al., Value of measuring serum procalcitonin, C-reactive protein, and mannan antigens to distinguish fungal from bacterial infections. Eur J Clin Microbiol Infect Dis, 2005. 24(4): p. 272-5.

14. Zahorec, R., Ratio of neutrophil to lymphocyte counts--rapid and simple parameter of systemic inflammation and stress in critically ill. Bratisl Lek Listy, 2001. 102(1): p. 5-14.

15. de Jager, C.P., et al., Lymphocytopenia and neutrophil-lymphocyte count ratio predict bacteremia better than conventional infection markers in an emergency care unit. Crit Care, 2010. 14(5): p. R192.

16. Huttunen, R., et al., Plasma level of soluble urokinase-type plasminogen activator receptor as a predictor of disease severity and case fatality in patients with bacteraemia: a prospective cohort study. J Intern Med, 2011. 270(1): p. 32-40.

17. Eugen-Olsen, J., suPAR - a future risk marker in bacteremia. J Intern Med, 2011. 270(1): p. 2931.

18. Schuetz, P., W. Albrich, and B. Mueller, Procalcitonin for diagnosis of infection and guide to antibiotic decisions: past, present and future. BMC Med, 2011. 9: p. 107.

19. Vincent, J.L., K. Donadello, and X. Schmit, Biomarkers in the critically ill patient: C-reactive protein. Crit Care Clin, 2011. 27(2): p. 241-51.

20. Dellinger, R.P., et al., Surviving Sepsis Campaign: international guidelines for management of severe sepsis and septic shock: 2008. Intensive Care Med, 2008. 34(1): p. 17-60. 
21. Levy, M.M., et al., The Surviving Sepsis Campaign: results of an international guideline-based performance improvement program targeting severe sepsis. Intensive Care Med, 2010. 36(2): p. 222-31.

22. Lodes, U., et al., PCR-based rapid sepsis diagnosis effectively guides clinical treatment in patients with new onset of SIRS. Langenbecks Arch Surg, 2012. 397(3): p. 447-55.

23. Fitting, C., et al., DNAemia Detection by Multiplex PCR and Biomarkers for Infection in Systemic Inflammatory Response Syndrome Patients. PLoS One, 2012. 7(6): p. e38916.

24. Hoenigl, M., et al., Diagnostic accuracy of soluble urokinase plasminogen activator receptor (sUPAR) for prediction of bacteremia in patients with systemic inflammatory response syndrome. Clin Biochem, 2013. 46(3): p. 225-9.

25. Zadroga, R., et al., Comparison of 2 blood culture media shows significant differences in bacterial recovery for patients on antimicrobial therapy. Clin Infect Dis, 2013. 56(6): p. 790-7.

26. Gogos, C.A., et al., Pro- versus anti-inflammatory cytokine profile in patients with severe sepsis: a marker for prognosis and future therapeutic options. J Infect Dis, 2000. 181(1): p. 176-80.

27. Mencacci, A., et al., Procalcitonin predicts real-time PCR results in blood samples from patients with suspected sepsis. PLoS One, 2012. 7(12): p. e53279.

28. Koch, A. and F. Tacke, Risk stratification and triage in the emergency department: has this become 'suPAR' easy? J Intern Med, 2012. 272(3): p. 243-6.

29. Schreiber, J., et al., Comparison of three different commercial PCR assays for the detection of pathogens in critically ill sepsis patients. Med Klin Intensivmed Notfmed, 2013. 108(4): p. 311318.

30. Peters, R.P., et al., Streptococcus pneumoniae DNA load in blood as a marker of infection in patients with community-acquired pneumonia. J Clin Microbiol, 2009. 47(10): p. 3308-12.

31. Andrade, S.S., P.J. Bispo, and A.C. Gales, Advances in the microbiological diagnosis of sepsis. Shock, 2008. 30 Suppl 1: p. 41-6.

32. Weile, J. and C. Knabbe, Current applications and future trends of molecular diagnostics in clinical bacteriology. Anal Bioanal Chem, 2009. 394(3): p. 731-42.

33. Mancini, N., et al., The era of molecular and other non-culture-based methods in diagnosis of sepsis. Clin Microbiol Rev, 2010. 23(1): p. 235-51.

34. Loonen, A.J., et al., Comparison of pathogen DNA isolation methods from large volumes of whole blood to improve molecular diagnosis of bloodstream infections. PLoS One, 2013. 8(8): p. e72349. 


\section{Chapter 9}

Tuf mRNA might be a promising marker to detect viable

Staphylococcus aureus in blood of bacteremic patients

Anne J.M. Loonen ${ }^{1-3}$, Petra F.G. Wolffs ${ }^{3}$, Maikel de Bresser ${ }^{1,2}$, Maurice Habraken ${ }^{1,2}$, Cathrien A. Bruggeman ${ }^{3}$, Mirjam H.A. Hermans', and Adriaan J.C. van den Brule ${ }^{1,2}$

1 Jeroen Bosch Hospital, Department of Medical Microbiology and Pathology, Laboratory for Molecular Diagnostics, 's-Hertogenbosch, The Netherlands

${ }^{2}$ Fontys University of Applied Sciences, Department of Medical Molecular Diagnostics, Eindhoven, The Netherlands

${ }_{3}^{3}$ Maastricht University Medical Centre, CAPHRI, Department of Medical Microbiology, Maastricht, The Netherlands 


\section{Abstract}

Bloodstream infections ( $\mathrm{BSI}$ ) pose serious health risks and can be life threatening. To detect the pathogen(s) involved and monitor BSI treatment most molecular methods to date focus on DNA. However, the analyses of RNA in BSI may have several advantages. Due to its shorter half-life, the presence of RNA may better relate to viability of pathogens, and due to its occurrence as multi-copy target, it may increase test sensitivity. We therefore investigated the presence of various nucleic acids targets (DNA, rRNA and mRNA) of Staphylococcus aureus during bacterial growth and antibiotic induced killing in relation to viability.

S. aureus was cultured to log phase and spiked in Todd Hewitt (TH) broth and whole blood of healthy human volunteers. Viability of $S$. aureus after flucloxacillin treatment $(0,1,3$ and 6 days) was assessed by culture on bloodagar plates. In addition, the presence of $S$. aureus DNA, rRNA, and mRNA were determined by real-time PCR of the $16 S$ rDNA and tuf gene.

S. aureus spiked in TH broth without antibiotics grew from day 0-6 and DNA (tuf and 16S), and $16 \mathrm{~S}$ rRNA remained detectable during this whole period. During flucloxacillin treatment $S$. aureus lost viability from day 3 onwards, while the $16 S$ rRNA-gene and its RNA transcripts remained detectable. However, tuf mRNA became undetectable from day 3 onwards. When spiking $S$. aureus in whole blood instead of broth no bacterial growth was seen, neither in the absence nor in the presence of flucloxacillin. Accordingly, no increase in DNA and RNA levels of both $16 S$ rDNA and the tuf gene were detected.

In conclusion, the results indicate that DNA and rRNA can be detected in flucloxacillin treated $S$. aureus cultures that do not further contain culturable bacteria. mRNA, in contrast, was only detectable from samples with culturable bacteria. Thus, mRNA might be a promising marker to measure active $S$. aureus bloodstream infection and possibly monitor antibiotic effects. 


\section{Introduction}

Bacteremia is defined as the presence of viable bacteria in the bloodstream [1]. The current gold standard method for the detection of microorganisms in the bloodstream is blood culture and subsequent identification of the bacteria by conventional (sometimes automated) biochemical techniques or MALDI-TOF MS [2-4]. An important advantage of this method is that only viable microorganisms are detected. A major disadvantage of the method is that the time-to-results is long (24-72 hours) due to the involvement of culturing steps. Because fast and accurate diagnosis is of crucial importance for patients suffering from BSI, molecular (real-time) PCR applications are increasingly being applied to decrease time to pathogen identification, thereby improving patient outcome [5-8]. However, all commercially available sepsis tests (SeptiFAST (Roche), SepsiTest (Molzym), and MagicPlex Sepsis Test (Seegene)) are based on DNA detection. DNA is a stable molecule and the presence of DNA of a certain pathogen does not provide information about the viability status of that pathogen as the DNA can originate from either living or dead pathogens [9-12]. In contrast to DNA, bacterial messenger RNA molecules have a half-live of only minutes [13]. For that reason, several studies have evaluated the detection of mRNA as a marker for the presence of actively growing bacteria $[9,11,14-18]$. Some of these studies have focused on detection of viable pathogens from food and environmental samples [14], while other studies focused on human disease and viability of pathogens from spiked culture broths (i.e. Borrelia burgdorferi, E. coli, Salmonella typhi, Shigella sonnei, Mycobacterium smegmatis) [10, 11, 17, 19]. Few studies used clinical specimens (Mycobacterium tuberculosis from sputum samples, Aspergillus spp from blood samples, Chlamydia trachomatis from cervical smears and urine) $[12,15,16]$. If RNA markers can be used to assess pathogen viability for BSI, the application of PCR based methods on RNA (cDNA) would be of great significance.

BSI can be caused by numerous pathogens [20]. In this study, the most commonly detected Gram-positive bacterium; S. aureus was chosen for reconstruction experiments. To investigate which nucleic acid molecule most favourably correlates to the viability status of S. aureus, DNA and rRNA of 16S rRNA gene, and DNA and mRNA levels of the tuf gene were measured in response to antibiotic therapy. The aim of this work was to find a suitable marker for $S$. aureus viability to be able to improve BSI diagnostics.

\section{Materials and Methods}

\section{Bacterial strain and growth conditions}

S. aureus (ATCC 25923) was used for reconstruction (spiking) experiments. Todd Hewitt $(\mathrm{TH})$ broth was inoculated with $\mathrm{S}$. aureus and cultured overnight at $35^{\circ} \mathrm{C}$. Subsequently, a 
1:100 dilution was made in fresh $\mathrm{TH}$ broth $(5 \mathrm{ml})$ for additional culturing to exponential phase (optical density 0.2 at $600 \mathrm{~nm}$; approximately $1 \times 10^{7}$ cells $/ \mathrm{ml}$ ).

\section{Spiking of S. aureus in TH broth and whole blood}

See Figure 1 for an overview of the experimental setup. S. aureus bacteria grown in exponential phase were diluted in either TH broth or pooled (of similar blood type, i.e. $\mathrm{O}+$ ), 1 day old, residual whole blood from healthy volunteers. The 100 and 10 colony forming units $(\mathrm{CFU}) / \mathrm{ml}$ dilutions were made in 2 tubes with an end volume of $10 \mathrm{ml} \mathrm{TH}$ broth or whole blood, respectively. To one tube an overdose of flucloxacillin (floxapen $5 \mu \mathrm{g} / \mathrm{ml}, 1$ $\mathrm{ml}$, Actavis, Baarn, the Netherlands) was added (to kill the bacteria) and to the other $1 \mathrm{ml}$ physiological salt solution, this was used as a control. Both tubes were placed on a shaker at room temperature. On days $0,1,3$, and 6, $200 \mu$ samples were taken from both tubes (flucloxacillin treated and untreated) for DNA and RNA isolation. Additionally, $100 \mu$ was taken to determine CFU/ml on blood agar plates (Tryptone Soya Agar with sheep blood, Oxoid Deutschland $\mathrm{GmbH}$, Wesel, Germany). Bacterial death was defined as the inability of producing colonies on bloodagar.

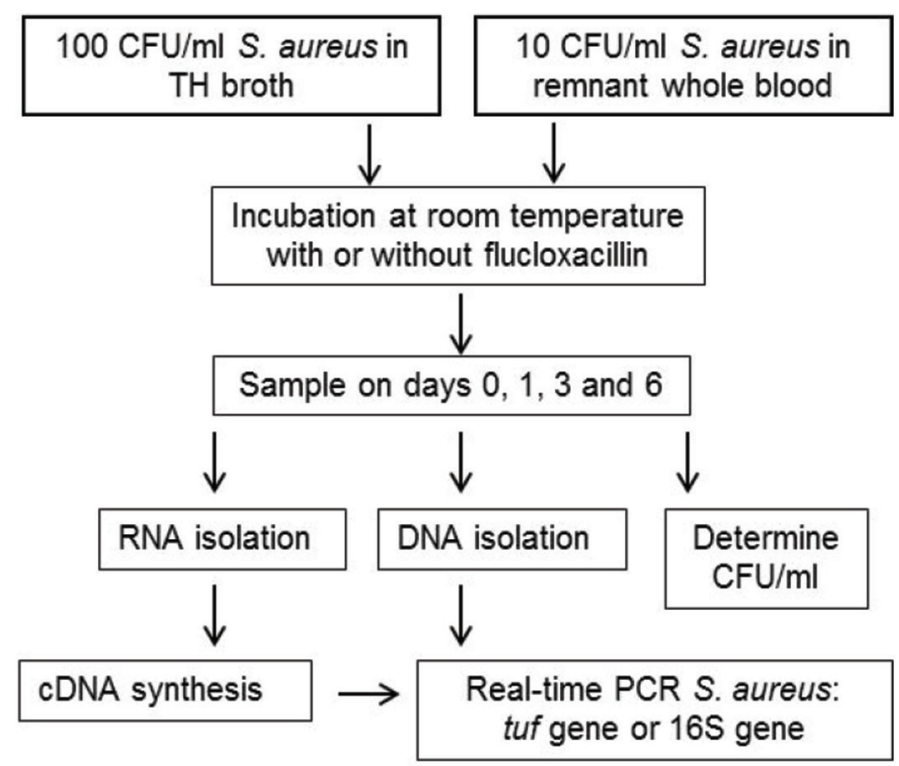

Figure 1. Overview of the experimental setup.

CFU, colony forming unit. 


\section{DNA and RNA isolation}

The obtained $200 \mu$ l samples (TH broth and whole blood) were centrifuged at $14.000 \mathrm{rpm}$ for $2 \mathrm{~min}$. The supernatant was removed and the pellet was washed once with $200 \mu \mathrm{l}$ ultra-pure water and centrifuged for 2 minutes at $14.000 \mathrm{rpm}$. The obtained pellet was resuspended in $20 \mu \mathrm{l}$ lysozym (12.5\%) and $75 \mu \mathrm{l}$ lysostaphin $(100 \mu \mathrm{g} / \mathrm{ml})$ and incubated for $30 \mathrm{~min}$ at $37^{\circ} \mathrm{C}$ while shaking (1000 rpm). RLT buffer with ß-mercaptoethanol (1:100) (Qiagen RNA blood mini kit) was added and the samples were stored at $-80^{\circ} \mathrm{C}$ until all time points were collected. The EasyMAG (BioMérieux, Marcy L'Etoile, France) was used for DNA isolation by using the specific B protocol. RNA was isolated by using the RNA blood mini kit (Qiagen), according to manufacturer's instructions. DNAse treatment was performed as described in the manual provided (Qiagen RNA blood mini kit) using columns to degrade the DNA in the samples.

\section{cDNA synthesis with random primers}

Reverse transcription was performed on RNA samples using the SuperScript ${ }^{\mathrm{TM}}$ II First-Strand Synthesis System for RT-PCR (Invitrogen, Carlsbad, CA, USA, according to manufacturer's protocol). Each sample was split in two for the plus and minus reverse transcriptase reaction to check DNA degradation (DNAse treatment on column).

\section{Real-Time PCR for tuf and 16S rRNA}

See Table 1 for an overview of the used primers and probes (tuf and 16S rDNA). The 16S rDNA primers, specific for most clinically relevant staphylococci, were described by Matsuda et al. [21]. However, the 16S rDNA forward primer was slightly modified to adapt to proper annealing temperature. An XS- probe (Biolegio, Nijmegen, The Netherlands) for Staphylococcus spp. detection based on $16 \mathrm{~S}$ was specifically designed. The PCR mix used has been described previously [22]. Additionally, tuf or $16 \mathrm{~S}$ primers (900 nM), tuf or $16 \mathrm{~S}$ probe $(200 \mathrm{nM})$, and $5 \mu \mathrm{l}$ sample were added to obtain an final volume of $20 \mu \mathrm{l}$. The PCR program used was described previously [23]. Both PCRs were run in white plates on the LightCycler 480 II (Roche Diagnostics).

Table 1. Primers and probes used in this study.

\begin{tabular}{|c|c|c|c|c|}
\hline Gene & Forward primer 5'-3' & Reverse primer 5'-3' & Probe 5'-3' & Reference \\
\hline tuf & $\begin{array}{l}\text { tcctggttcaattacaccacat- } \\
\text { actg }\end{array}$ & $\begin{array}{l}\text { ggaaatagaattgtggacga- } \\
\text { tagtttga }\end{array}$ & $\begin{array}{l}\text { FAM- tgata- } \\
\text { atacrtawacttctgc-BHQ1 }\end{array}$ & [23] \\
\hline $16 S$ & acggtcttgctgtcactta & tacacatatgttcttccctaataa & $\begin{array}{l}\text { VIC-gtaacggcttaccaag- } \\
\text { gc-BHQ1 }\end{array}$ & [21] \\
\hline
\end{tabular}




\section{Results}

\section{Detection of S. aureus DNA, rRNA, and mRNA after antibiotic treatment from TH broth}

In absence of flucloxacillin the $S$. aureus bacteria continued to grow. At day 0 , on average $330 \pm 28$ (average \pm SD) CFU/ml were detected on bloodagar plates (Table 2). At days 1, 3, and 6 the $\mathrm{CFU} / \mathrm{ml}$ increased to $>1000 \mathrm{CFU} / \mathrm{ml}$. In contrast, bacterial growth was arrested in flucloxacillin (antibiotic) treated samples and no colonies were detected on bloodagar at days 3 and 6 .

Simultaneously, samples were taken for DNA and RNA isolation. In the absence of flucloxacillin, Ct values of both 16S (DNA and rRNA) and tuf (DNA and mRNA) decreased in time (Figure 2). In the presence of flucloxacillin, DNA of the 16S rDNA gene and the tuf gene were detected until day 6 , while bloodagar plates indicated absence of culturable $S$. aureus on day 3. 16S rRNA also remained detectable up to 6 days of treatment. However, tuf mRNA could not be detected on days 3 and 6 . The data indicate that $S$. aureus DNA and rRNA can still persist in the absence of viable bacteria as demonstrated using culture.

The $\mathrm{Ct}$ values obtained on day zero are similar for both 16S DNA and rRNA with(out) flucloxacillin. This is not true for tuf DNA and mRNA. Ct values obtained for tuf mRNA are on average $2 \mathrm{Ct}$ higher as compared to tuf DNA (day 0 ).

These results demonstrate that tuf mRNA is the nucleic acid target that could only be detected from samples which contain culturable bacteria. DNA and rRNA targets could be detected in flucloxacillin treated $S$. aureus cultures that do not further contain culturable bacteria. This experiment was performed twice on independent days, and showed similar results.

\section{S. aureus viability measurements from spiked whole blood}

In order to mimic a bloodstream infection, whole blood samples instead of $\mathrm{TH}$ broth were spiked with log phase $S$. aureus and growth was measured both in presence and absence

Table 2. Plate counts of Staphylococcus aureus samples with or without flucloxacillin treatment from TH broth.

\begin{tabular}{clllll}
\hline & Flucloxacillin & Yes & No & Yes & No \\
\hline \multirow{4}{*}{ Days } & & Average & SD & Average & SD \\
& 0 & 290 & 14 & 330 & 28 \\
& 1 & 255 & 7 & $\infty$ & ND \\
& 3 & 0 & 0 & $\infty$ & ND \\
6 & 0 & 0 & $\infty$ & ND \\
\hline
\end{tabular}

Numbers represent colony forming units/ml

$\infty$; uncountable plate due to large amount of colonies, ND; not determined 


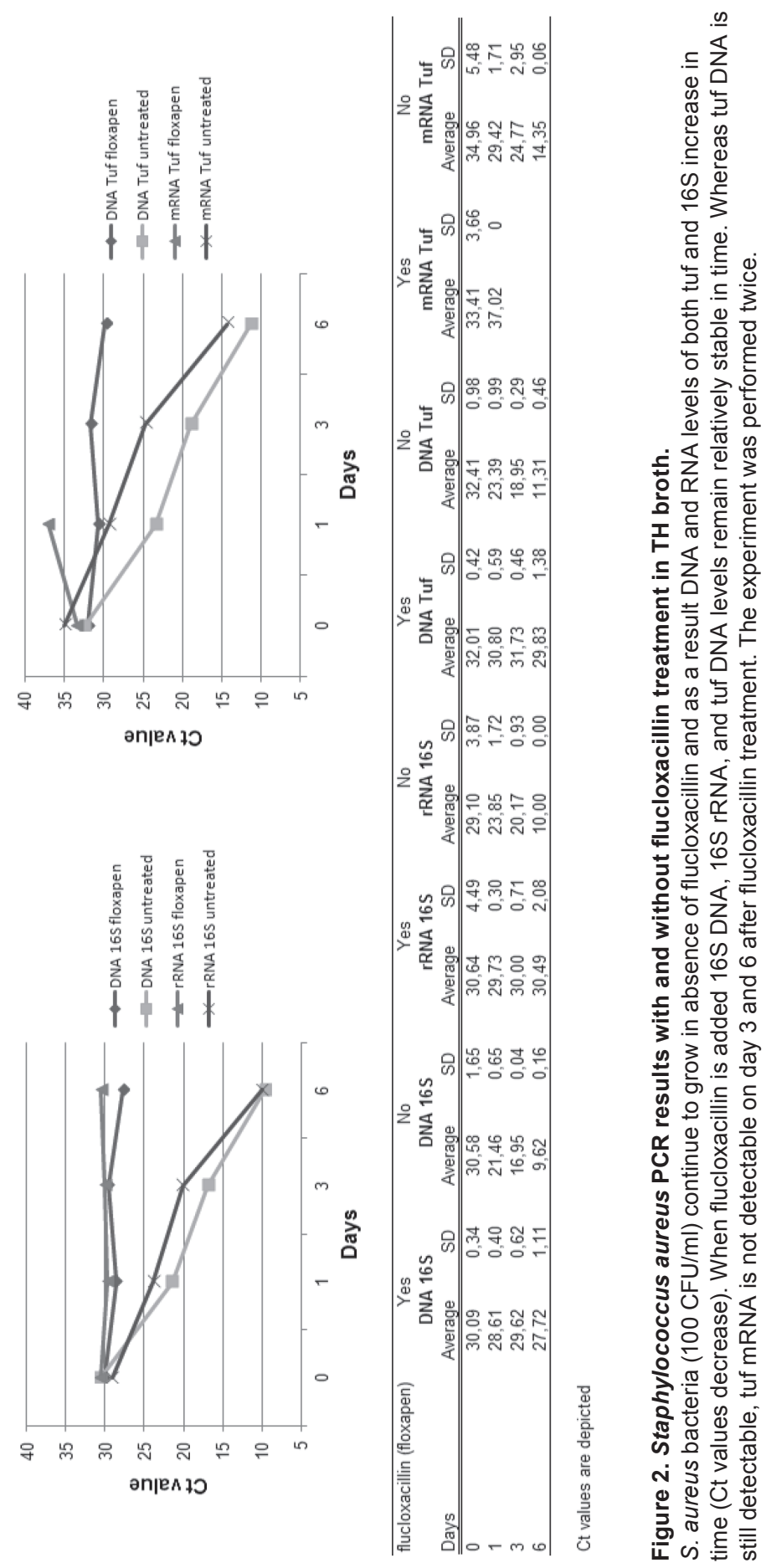




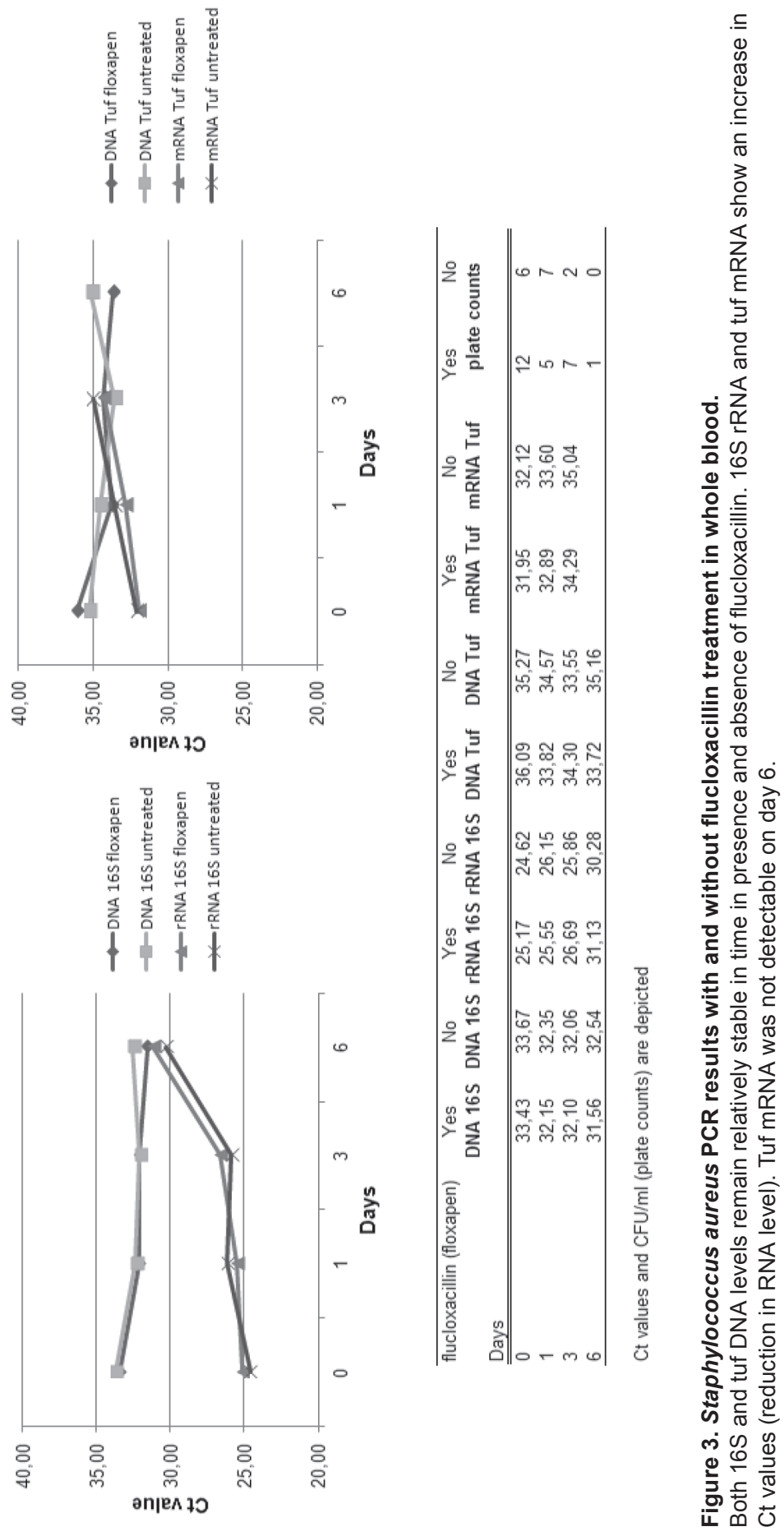


of flucloxacillin. Furthermore, a more clinical significant initial bacterial load was used of 10 $\mathrm{CFU} / \mathrm{ml}$ (instead of $100 \mathrm{CFU} / \mathrm{ml}$ ). The plate counts (Figure 3) show that in both presence and absence of flucloxacillin bacterial numbers decrease. DNA and RNA measurements of $16 \mathrm{~S}$ and tuf also showed growth arrest, both in presence and absence of flucloxacillin, as no decrease in Ct values was observed in time (0-6 days) (Figure 3), this in contrast to the results obtained by using spiked TH broth in absence of flucloxacillin (Figure 2).

The $\mathrm{Ct}$ values obtained by detecting 16S DNA and rRNA are lower as compared to the $\mathrm{Ct}$ values for tuf DNA and mRNA. A difference of at least $6 \mathrm{Ct}$ was observed when comparing 16S rRNA levels to tuf mRNA levels. When comparing Ct values for DNA detection of both genes the differences were less pronounced, but still significant (approximately $3 \mathrm{Ct}$ ). A clear difference was observed between the spiked TH broth samples (Figure 2) and the spiked whole blood samples at day zero (Figure 3). Ct values for 16S DNA and rRNA were comparable in TH broth (day 0), but not in whole blood. For tuf DNA and mRNA this phenomenon was also observed, in whole blood the Ct values for tuf mRNA were lower than for tuf DNA (day 0). Furthermore, there seemed to be a trend towards higher $\mathrm{Ct}$ values from day $0-6$, independent of flucloxacillin, for both $16 \mathrm{~S}$ rRNA and tuf mRNA in whole blood. This confirms the culture results obtained from whole blood.

In addition, it was investigated whether fresh (max. 1 hour) and residual (1 day old) whole blood differed in their performances as medium for bacterial culture. TH broth was used as a control: in the absence of flucloxacillin large amounts of colonies were detected up to 6 days of culture, while in the presence of flucloxacillin colonies were detected on days 0 and 1 , and no colonies were detected on bloodagar plates on days 3 and 6 (Table 3). Plate counts from spiked blood samples did not resemble those obtained from spiked TH broth samples. The initial plate count on day 0 , while initiated from the same log phase culture, was half or less when compared to the count on day 0 for TH broth samples. However, no significant differences were observed between fresh and residual blood samples both in presence or absence of flucloxacillin.

Table 3. Plate counts of Staphylococcus aureus with or without flucloxacillin treatment from TH broth, fresh whole blood ( $1 \mathrm{~h}$ ) and remnant whole blood (1 day).

\begin{tabular}{llllllll}
\hline & & \multicolumn{2}{l}{ TH broth } & \multicolumn{2}{l}{ Fresh whole blood } & \multicolumn{2}{l}{$\begin{array}{l}\text { Remnant whole } \\
\text { blood }\end{array}$} \\
\hline Flucloxacillin & & Yes & No & Yes & No & Yes & No \\
Days & $\mathbf{0}$ & 20 & 22 & 10 & 4 & 3 & 6 \\
& 1 & 14 & $\infty$ & 2 & 3 & 1 & 1 \\
& $\mathbf{3}$ & 0 & $\infty$ & 2 & 1 & 0 & 3 \\
& $\mathbf{6}$ & 0 & $\infty$ & 1 & 0 & 0 & 0 \\
\hline
\end{tabular}

Numbers represent colony forming units/ml

$\infty$; uncountable plate due to large amount of colonies 


\section{Discussion}

For patient survival it is important to provide fast and accurate identification of BSI causing pathogens. Only viable microorganisms can be cultured, and fastidious or damaged organisms can be present in whole blood but often remain culture negative. Molecular diagnostics might provide solutions for these problems, as pathogens in antibiotic treated patients who remain culture negative (due to presence of antibiotics in the bloodstream) can be identified by PCR $[24,25]$. As BSI is defined as the presence of viable pathogens in the bloodstream, it might be important for a molecular assay to allow pathogen viability measurements from whole blood.

The results that were obtained after spiking $S$. aureus in $\mathrm{TH}$ broth, with and without flucloxacillin, indicated that tuf mRNA might be a more promising marker to measure viability than DNA and rRNA. Tuf mRNA levels correlated with the culture results from both TH broth and whole blood, whereas 16S DNA, 16S rRNA and tuf DNA levels did not. These data confirm results from previous studies $[9,10,15,18]$.

The $S$. aureus bacteria used in this study seemed to die in whole blood (growth reduction on agar plates in absence of flucloxacillin) or enter a state in which they are viable but nonculturable (VBNC) [26-28]. Bacteria enter the VBNC state in response to stress, such as starvation, incubation outside the growth temperature range, or oxygen concentration [28]. In this study, several stressful conditions might have been present. S. aureus was cultured in whole blood in which white cells might inhibit bacterial growth [29]. Additionally, incubation took place at room temperature (RT) for 6 days, and waste products were not removed from the culture tube. In future studies, it might be useful to remove the white blood cells from whole blood before spiking (buffy coat), and incubate the samples at $35^{\circ} \mathrm{C}$ instead of RT to create better growth conditions. Furthermore, different $S$. aureus strains need to be tested to confirm our results.

In this study, bacteria were considered dead when they were unable to produce colonies on bloodagar. However, as mentioned before, bacteria can enter a viable by non-culturable state (VBNC) under stressful conditions [26, 30]. Bacteria that are not culturable can potentially still be viable and infective. A limitation of this study is that bacterial viability was only measured by colony formation on bloodagar plates. In future studies additional methods to assess bacterial viability might be included, for instance the Live/Dead BacLight Bacterial Viability Kit (Invitrogen). This kit provides two nucleic acid stains (green-fluorescent SYTO 9 dye and red-fluorescent propidium iodide $(\mathrm{PI})$ ) to be able distinguish live bacteria (intact membranes) from dead bacteria (compromised membranes). PI is a cell membrane impermeable dye and can only enter compromised pathogens [31]. Another option to differentiate live from dead pathogens is exposure to the dye propidium monoazide (PMA) followed by real-time PCR. 
PMA cannot penetrate viable cells with intact cytoplasmic membranes [32]. The PMA dye can enter dead pathogens and bind DNA, thereby inhibiting PCR amplification.

It has clearly been demonstrated that tuf mRNA mostly resembles culture results (in both $\mathrm{TH}$ broth and whole blood). Both DNA and rRNA remain detectable even when culture results are negative. The $\mathrm{Ct}$ values obtained for $S$. aureus spiked in $\mathrm{TH}$ broth are different from those in the whole blood. Because a lower amount of $S$. aureus bacteria (10 CFU/ml) was spiked in whole blood, as compared to TH broth (100 CFU/ml), one would expect the Ct value to be 3,3 $(1 \mathrm{log})$ higher in whole blood samples. This difference of approximately $3 \mathrm{Ct}$ was seen in whole blood as compared to TH broth for DNA (both $16 \mathrm{~S}$ and tuf). However, the $\mathrm{Ct}$ values obtained for tuf mRNA and 16S rRNA ( $t=0)$ were higher in TH broth as compared to whole blood (approximately 2 and $5 \mathrm{Ct}$, respectively). Both RNA targets (tuf and 16S) seem to be expressed at a higher level in whole blood. This unexpected phenomenon might be a result of the difference in environment (blood versus broth). This confirms findings reported by Cenciarini et al. who showed that it is difficult to compare RNA viability markers for one pathogen kept in different conditions [14].

In this study, detection of mRNA and rRNA was performed by using reverse-transcription real-time PCR (RT-PCR). Birch et al. investigated the use of PCR, RT-PCR and nucleic acid sequence based amplification (NASBA) for assessment of bacterial viability [33]. They found that NASBA offered the highest sensitivity of the three methods tested. However, presence of residual fliC DNA and mRNA could be detected by NASBA 30 hours post-death (culture negative). Other studies have shown that RNA detection by NASBA could be used to monitor infections after antibiotic treatment $[12,19]$. These contradictory findings again demonstrate that it is important to thoroughly investigate which RNA target is suitable for viability measurement of a certain pathogen. In future studies, it would be interesting to use the NASBA technology in addition to RT-PCR.

In this study, results were obtained from as little as $200 \mu$ whole blood. Larger volumes of blood are needed to be able to detect clinical relevant bacterial loads [34]. As bacterial enrichment is a prerequisite to be able to detect bacteria from whole blood, RNA isolation methods should include such an approach. Both Polaris (Biocartis, Mechelen, Belgium) and MolYsis (Molzym GmbH, Bremen, Germany) have developed suitable techniques for pathogen DNA enrichment from large volumes of blood [34]. However, these enrichment strategies are not suitable for RNA isolation. A small pilot study indicated that the first steps, of both the MolYsis and the Polaris pathogen enrichment methods, in which human cells and DNA were removed, did not kill the pathogens present in the whole blood samples as shown by positive cultures (data not shown). It would be interesting to investigate if enrichment of pathogens, with either Polaris or MolYsis, can be combined with existing RNA isolation procedures to obtain pathogen RNA from large volumes $(5 \mathrm{ml})$ of blood. 
Several studies have to date been published which describe the superiority of a mRNA target in the detection of viable bacteria $[12,15-17,33]$. BSI can be the result of a broad scala of pathogens and, in this study, $S$. aureus was chosen for initial reconstruction experiments to show proof of principle. At present several commercial sepsis tests are available and it remains to be seen if these can be used for pathogen identification and simultaneous assessment of viability.

In conclusion, this study clearly demonstrated that detection of $S$. aureus tuf mRNA, in contrast to DNA and rRNA, correlates to bacterial viability status as determined by culture. Therefore, tuf mRNA might be a promising marker to measure active $S$. aureus bloodstream infection. After development of RNA isolation procedures from large volumes of whole blood, future clinical studies are needed to validate the preliminary findings obtained in this study. 


\section{References}

1. M, S., in Stedman's Medical Dictionary1995, Md: Lippincott Williams \& Wilkins: Baltimore.

2. Loonen, A.J., et al., An evaluation of three processing methods and the effect of reduced culture times for faster direct identification of pathogens from BacT/ALERT blood cultures by MALDI-TOF MS. Eur J Clin Microbiol Infect Dis, 2012. 31(7): p. 1575-83.

3. Riedel, S. and K.C. Carroll, Blood cultures: key elements for best practices and future directions. J Infect Chemother, 2010. 16(5): p. 301-16.

4. van Veen, S.Q., E.C. Claas, and E.J. Kuijper, High-throughput identification of bacteria and yeast by matrix-assisted laser desorption ionization-time of flight mass spectrometry in conventional medical microbiology laboratories. J Clin Microbiol, 2010. 48(3): p. 900-7.

5. Fraser, A., et al., Benefit of appropriate empirical antibiotic treatment: thirty-day mortality and duration of hospital stay. Am J Med, 2006. 119(11): p. 970-6.

6. Gaibani, P., et al., Blood culture systems: rapid detection--how and why? Int J Antimicrob Agents, 2009. 34 Suppl 4: p. S13-5.

7. Wallet, F., et al., Preliminary clinical study using a multiplex real-time PCR test for the detection of bacterial and fungal DNA directly in blood. Clin Microbiol Infect, 2010. 16(6): p. 774-779.

8. Yanagihara, K., et al., Evaluation of pathogen detection from clinical samples by real-time polymerase chain reaction using a sepsis pathogen DNA detection kit. Crit Care, 2010. 14(4): p. R159.

9. Hellyer, T.J., et al., Detection of viable Mycobacterium tuberculosis by reverse transcriptasestrand displacement amplification of mRNA. J Clin Microbiol, 1999. 37(3): p. 518-23.

10. Iyer, R., et al., Detection of Borrelia burgdorferi Nucleic Acids after Antibiotic Treatment Does Not Confirm Viability. J Clin Microbiol, 2013. 51(3): p. 857-62.

11. Josephson, K.L., C.P. Gerba, and I.L. Pepper, Polymerase chain reaction detection of nonviable bacterial pathogens. Appl Environ Microbiol, 1993. 59(10): p. 3513-5.

12. Morre, S.A., et al., Monitoring of Chlamydia trachomatis infections after antibiotic treatment using RNA detection by nucleic acid sequence based amplification. Mol Pathol, 1998. 51(3): p. 149-54.

13. Arraiano, C.M., S.D. Yancey, and S.R. Kushner, Stabilization of discrete mRNA breakdown products in ams pnp rnb multiple mutants of Escherichia coli K-12. J Bacteriol, 1988. 170(10): p. 4625-33.

14. Cenciarini, C., et al., Influence of long time storage in mineral water on RNA stability of Pseudomonas aeruginosa and Escherichia coli after heat inactivation. PLoS One, 2008. 3(10): p. e3443.

15. Jou, N.T., et al., Single-tube, nested, reverse transcriptase PCR for detection of viable Mycobacterium tuberculosis. J Clin Microbiol, 1997. 35(5): p. 1161-5.

16. Loeffler, J., et al., Nucleic acid sequence-based amplification of Aspergillus RNA in blood samples. J Clin Microbiol, 2001. 39(4): p. 1626-9.

17. Sheridan, G.E., et al., Detection of $m R N A$ by reverse transcription-PCR as an indicator of viability in Escherichia coli cells. Appl Environ Microbiol, 1998. 64(4): p. 1313-8.

18. Simpkins, S.A., et al., An RNA transcription-based amplification technique (NASBA) for the detection of viable Salmonella enterica. Lett Appl Microbiol, 2000. 30(1): p. 75-9.

19. van der Vliet, G.M., et al., Assessment of mycobacterial viability by RNA amplification. Antimicrob Agents Chemother, 1994. 38(9): p. 1959-65.

20. Wisplinghoff, H., et al., Nosocomial bloodstream infections in US hospitals: analysis of 24,179 cases from a prospective nationwide surveillance study. Clin Infect Dis, 2004. 39(3): p. 309-17.

21. Matsuda, K., et al., Sensitive quantitative detection of commensal bacteria by rRNA-targeted reverse transcription-PCR. Appl Environ Microbiol, 2007. 73(1): p. 32-9.

22. Huijsmans, C.J., et al., Comparative analysis of four methods to extract DNA from paraffinembedded tissues: effect on downstream molecular applications. BMC Res Notes, 2010. 3: p. 239. 
23. Loonen, A.J., et al., Acceleration of the direct identification of Staphylococcus aureus versus coagulase-negative staphylococci from blood culture material: a comparison of six bacterial DNA extraction methods. Eur J Clin Microbiol Infect Dis, 2011. 30(3): p. 337-42.

24. Mauro, M.V., et al., Diagnostic utility of LightCycler SeptiFast and procalcitonin assays in the diagnosis of bloodstream infection in immunocompromised patients. Diagn Microbiol Infect Dis, 2012. 73(4): p. 308-11.

25. Wellinghausen, N., et al., Diagnosis of bacteremia in whole-blood samples by use of a commercial universal 16S rRNA gene-based PCR and sequence analysis. J Clin Microbiol, 2009. 47(9): p. 2759-65.

26. Davey, H.M., Life, death, and in-between: meanings and methods in microbiology. Appl Environ Microbiol, 2011. 77(16): p. 5571-6.

27. Keer, J.T. and L. Birch, Molecular methods for the assessment of bacterial viability. J Microbiol Methods, 2003. 53(2): p. 175-83.

28. Oliver, J.D., The viable but nonculturable state in bacteria. J Microbiol, 2005. 43: p. 93-100.

29. Hogman, C.F., et al., White cells protect donor blood against bacterial contamination. Transfusion, 1991. 31(7): p. 620-6.

30. Trevors, J.T., Can dead bacterial cells be defined and are genes expressed after cell death? J Microbiol Methods, 2012

31. Deligeorgiev, T.G., S. Kaloyanova, and J.J. Vaquero, Intercalating Cyanine Dyes for Nucleic Acid Detection. Recent Patents on Materials Science, 2009(2): p. 1-26.

32. Nocker, A., C.Y. Cheung, and A.K. Camper, Comparison of propidium monoazide with ethidium monoazide for differentiation of live vs. dead bacteria by selective removal of DNA from dead cells. J Microbiol Methods, 2006. 67(2): p. 310-20.

33. Birch, L., et al., A comparison of nucleic acid amplification techniques for the assessment of bacterial viability. Lett Appl Microbiol, 2001. 33(4): p. 296-301.

34. Loonen, A.J., et al., Comparison of pathogen DNA isolation methods from large volumes of whole blood to improve molecular diagnosis of bloodstream infections. PLoS One, 2013. 8(8): p. e72349. 


\section{Chapter 10}

Summary, General Discussion and Future Perspectives 
Bloodstream infections (BSIs) still remain increasing in incidence [1-6]. BSI is associated with high mortality and increased healthcare costs [7, 8]. Optimal management of BSI is dependent on several factors including recognition of the disease, laboratory tests and treatment. Rapid and accurate identification of the etiologic agent is crucial to be able to initiate pathogen specific antibiotic therapy and decrease mortality rates $[9,10]$. In case of the most serious bloodstream infections associated with septic shock, time is a crucial factor. The survival rate of severe sepsis decreases from $80 \%$ if appropriate therapy is given within one hour to less than $10 \%$ if effective therapy is not provided within 24 hours [11]. Furthermore, appropriate treatment might slow down the emergence of antibiotic resistant strains.

Culture-based methods are still considered to be the "gold standard" for the detection and identification of pathogens causing BSI. When blood cultures are detected as positive by the automated culture system, Gram staining is performed followed by subculture on solid media, and (semi-automated) biochemical testing for species identification. Finally, a complete antibiotic susceptibility profile can be provided based on cultured colonies, which allows the start of pathogen-tailored antibiotic therapy (Figure 1).

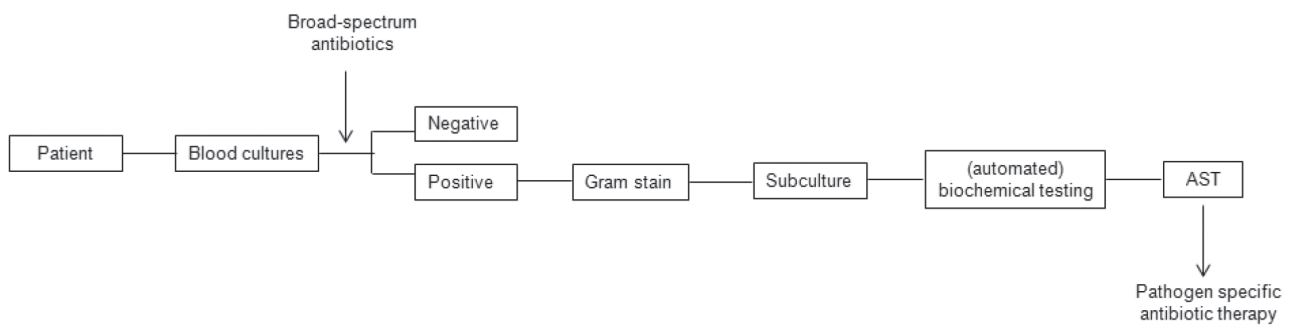

Figure 1. Overview of the conventional diagnostic process for BSI.

When blood culture positivity is detected by the automated culture system, Gram staining is performed, followed by subculture on agar media, and biochemical testing for pathogen identification. A complete antibiotic susceptibility profile is usually obtained by analysing growth inhibition on solid media. AST; antibiotic susceptibility testing.

This conventional workflow is extremely time-consuming and can take up several days. Furthermore, fastidious and slow-growing microorganisms, as well as antibiotic pre-treated samples can be falsely negative. The main aim of this thesis was to study different strategies to improve the conventional laboratory diagnostic steps for BSI (Figure 1). These approaches include protein-based (MALDI-TOF mass spectrometry) and nucleic acid-based (polymerase chain reaction $(\mathrm{PCR})$ ) identification from subculture, blood cultures, and whole blood to decrease time-to results. In addition, also pathogen enrichment and DNA isolation methods, to enable optimal pathogen DNA recovery from these clinical specimens, were investigated. 


\subsection{BSI diagnostics from blood cultures and subcultures}

\section{Polymerase chain reaction}

Gram-positive bacteria (i.e. S. aureus, CoNS, and Enterococcus spp.) are frequently recovered pathogens from blood cultures [12]. Therefore, we developed two real-time PCR duplex assays for detection of $S$. aureus, CoNS, and Enterococcus spp. Subsequently these assays were clinically evaluated by using; 1) growth positive blood cultures containing Grampositive cocci, and 2) one $\mathrm{ml}$ residual whole blood from patients at the intensive care unit (ICU) (Chapter 2). The duplex assays, based on the tuf gene, enabled fast and accurate detection from positive blood cultures. In a small pilot study using one $\mathrm{ml}$ residual whole blood samples of suspected BSI patients from the intensive care unit (ICU), the developed PCR assays combined with a sensitive bacterial DNA isolation method have shown potential value. In 18 out of the 22 patients (82\%) identical results were found when comparing blood culture and PCR.

Simultaneously, we investigated the possibility of reduced blood culture incubation in the automated culture system (Chapter 3). Six different bacterial DNA extraction protocols were compared to be able to find the best method for pathogen DNA isolation from blood cultures. This method was used to isolate pathogen DNA, and subsequently PCR was performed on blood culture bottles after only a few hours of culture. The MolYsis Plus kit (Molzym) in combination with the specific B protocol of the EasyMAG resulted in the best isolation of bacterial DNA from BacT/ALERT (bioMérieux) blood culture bottles. After initial spiking experiments (S. aureus) a detection limit of $10 \mathrm{CFU} / \mathrm{ml}$ could be detected by using the developed tuf based PCR. BACTEC (BD) blood culture bottles resulted in an one log lower detection limit. After an incubation step of only 5 hours $S$. aureus and CoNS could be detected from BacT/ALERT blood culture material by using the in-house PCR assay. By using this reduced incubation time it was possible to identify $S$. aureus and CoNS within one working day. Results were available at least 19 hours faster as compared to the conventional diagnostic process. In a study performed by Gebert et al. [13] similar results were reported. They showed a decrease of 5.0-8.7 hours in spiking experiments and an average time gain of 10.7 hours was obtained when the method was applied on true clinical specimens. Laboratories with a 24 hours setup will benefit most from this approach. More research is needed to determine which incubation time is suitable for most encountered pathogens. The tuf gene based real-time PCR described is only valuable for patients suspected of having a Staphylococcus spp. or Enterococcus spp. bloodstream infection. As BSI can be caused by a variety of pathogens, species-specific PCRs seem less valuable when optimal time-to results are required. The developed tuf gene based real-time PCR can be extended with other targets to be able to detect the most frequently cultured pathogens from blood cultures. This flexibility allows that the assay can be adjusted to the local epidemiology depending on 
the predominant patient group, i.e. covering pathogens found most frequently at a certain department or in a specific patient population [12,14]. Future studies could also make use of e.g. the multi-probe assay as described by Hansen et al. [15] or the commercially available Prove-it Sepsis test (MobiDiag, Helsinki, Finland) [16]. However, as both of these tests are developed for pathogen identification from growth positive blood cultures it remains to be investigated whether they are sensitive enough to be used on blood culture bottles which are not yet growth positive.

\section{MALDI-TOF MS}

Infections due to Gram-positive organisms are one of the leading causes of morbidity and mortality. A US study showed a high prevalence of Gram-positive bacteria in BSI patients; the most frequently isolated pathogens were CoNS (31\%) [12]. CoNS are normal inhabitants of human skin and mucous membranes. They have long been considered as culture contaminants, but as they are increasingly cultured, the role of CoNS as pathogens needs further investigation. Consequently, rapid and reliable determination of CoNS to the species level might be clinically relevant. Phenotypic methods, according to Kloos and Schleifer, have long been the "gold standard" for identification of CoNS to the species level [17]. The API ID 32 Staph strip (bioMérieux) and the automated VITEK 2 (bioMérieux) have improved phenotypic identification of CoNS spp. Furthermore, a variety of genes, 16S rRNA [18], sodA [19], and tuf [20, 21] are described to be suitable targets for genotypic CoNS spp. identification. Matrix Assisted Laser Desorption Ionisation- Time of Flight mass spectrometry (MALDI-TOF MS) allows examination of protein profiles from bacteria, and has been shown to work in a fast and accurate manner $[22,23]$. To be able to find the most suitable method for fast CoNS identification we compared five methods i.e. the VITEK 2 (bioMérieux), the ID 32 Staph strip (bioMérieux), partial 16S rDNA gene sequencing (MicroSeq, Applied Biosystems), partial tuf gene sequencing (in-house), and MALDI-TOF MS (Bruker Daltonics) on the same set of CoNS strains (Chapter 4). The results obtained in this study demonstrated the good performance of MALDI-TOF MS for identification of CoNS, a correct identification rate of $99.3 \%$ was achieved. Tuf gene sequencing is the most suitable alternative for MALDITOF MS. Heikens et al. also demonstrated that tuf gene sequencing is a good approach for identification of CoNS (5/57 incorrect ID) [20]. When fast and accurate identification of CoNS is needed, MALDI-TOF MS is the method of choice. This has also been demonstrated by others $[24,25]$.

It remains a challenge in daily practice to distinguish clinically significant CoNS from contaminant strains. As the most optimal method for CoNS identification was found to be MALDI-TOF MS, this technique was employed to study the clinical relevance of CoNS speciation (Chapter 5). Most CoNS infections are a result of the use of medical devices such as prosthetic devices and (intravenous) catheters [26]. However, S. lugdunensis 
and S. schleiferi have been reported as etiologic agents of BSI and endocarditis, and S. saprophyticus as a cause of urinary tract infections [27]. We have shown that speciation of CoNS could be helpful in establishing whether a patient is suffering from an actual CoNS bloodstream infection. The CoNS species most commonly found in bloodstream infections was S. epidermidis. However, in our study, S. epidermidis was also the most common blood culture contaminant. In addition, we found that $S$. haemolyticus was very often associated with BSI, whereas others were mostly found to be contaminants. This means that CoNS identification can be of clinical value to differentiate true pathogens from contaminants. CoNS speciation combined with the opinion of the clinical microbiologist might prove to be optimal for patient care. Moreover, molecular diagnostic tests should be able to detect the most clinically relevant CoNS species and more in-depth prospective studies are needed to confirm our findings.

More rapid direct identification of microorganisms from positive cultures can have major impact in the clinic. Therefore, we investigated the possibility of direct identification of bacteria from positive aerobe BacT/ALERT blood cultures with MALDI-TOF MS. In addition, it was evaluated if reduced time-to-identification from blood cultures could be achieved with reduced incubation times ( 7 hours) of $S$. aureus-and E. coli-spiked BacT/ALERT blood cultures (Chapter 6). When blood cultures are used in combination with MALDI-TOF MS, human cells need to be removed from the specimen to eliminate interference from human proteins in the analysis. Therefore, different protocols were analysed for their performance in bacterial enrichment and removal of these aspecific proteins. Our results showed that bacterial identification can be available within 20 min by using SepsiTyper (Bruker Daltonics), but also depends on the number of positive cultures that need to be processed. In contrast, the conventional workflow needs approximately 1-2 days to be able to report the bacterial species from positive blood cultures. Reduced incubation $(7 \mathrm{~h})$ of $S$. aureus-and $E$. colispiked aerobe BacT/ALERT cultures did not result in reliable identification. An alternative to the proposed workflow of using positive blood cultures for MALDI-TOF MS identification is the use of spot subculture on agar plates. This subculture entails culturing of positive blood culture material for approximately four hours. In general, after four hours enough bacteria will be available for MALDI-TOF MS analysis via the direct smear method (colonies from solid media). Blood cultures which become positive in the morning can be identified by this spot subculture. Pathogen identification with MALDI-TOF MS, directly from positive blood cultures can be performed on blood cultures which become growth positive later during the day. Szabados et al. reported low sensitivity (30\%) for MALDI-TOF MS for direct identification from positive BacT/ALERT blood culture bottles [28]. But then again, others have demonstrated the successful use of positive blood cultures, both BACTEC as well as BacT/ALERT bottles, as starting point for pathogen identification with MALDI-TOF MS [29-35]. 
MALDI-TOF MS can decrease time-to identification. However, it has been shown that a microbial load of $10^{7}-10^{8} \mathrm{CFU} / \mathrm{ml}$ is necessary to obtain protein spectra [29]. This indicates that pre-culture steps remain necessary for optimal results. Furthermore, it is not yet possible to use MALDI-TOF MS for antibiotic susceptibility testing. The first steps towards antibiotic susceptibility testing with MALDI-TOF MS have been undertaken [36, 37], but it is not possible to provide a complete AST profile yet.

\subsection{BSI diagnostics directly from whole blood}

\section{Sample preparation}

Pathogen detection and identification from whole blood is the only way to circumvent culture steps. Sample preparation is crucial to be able to remove human background cells and DNA, and to simultaneously enrich pathogens to detect the low bacterial loads [38].

Detection of pathogen DNA from whole blood is a promising approach for rapid diagnosis of BSI, permitting an earlier start of pathogen specific therapy. This is important, as it has been described that the use of inappropriate antimicrobial therapy can result in increased mortality [10,39]. In addition, misuse and incorrect administration of antibiotics have led to the development of resistant bacterial strains, and this results in adverse patient outcome [40]. In order to reach similar sensitivities as blood cultures, several pathogen DNA enrichment strategies were evaluated (Chapter 7). Spiking experiments, using S. aureus, Pseudomonas aeruginosa, and Candida albicans, indicated that both Polaris and MolYsis enrichment followed by DNA isolation and species-specific singleplex real-time PCRs enabled reliable and sensitive detection of bacteria and fungi from $5 \mathrm{ml}$ blood. Because the EasyMAG can only process $200 \mu \mathrm{l}$ whole blood, the detection rates with this method were not satisfactory. Polaris was most sensitive (highest detection rates, $100 \%$ for all samples containing $10 \mathrm{CFU} /$ $\mathrm{ml}$ ) and results were available within 3 hours.

In our study, both the MolYsis and the Polaris method removed sufficient human background DNA. No interference with the specific pathogen PCRs was detected. It has been shown by others that selective lysis of blood cells and the subsequent (non)-enzymatic degradation of released human DNA ensures a more sensitive detection of pathogen DNA [41, 42]. Handschur et al, showed, using total DNA extracts from whole blood, unspecific signals occurred in general 16S rDNA PCRs as a result of the amplification of human sequences [41]. Therefore, they developed an enrichment method for the analysis of blood samples. Clinical samples (whole blood) showed similar results as compared to standard blood culture methods. The enrichment method allowed specific and sensitive detection of pathogens in PCR assays. Hansen et al. have shown that the combination of performing an enrichment step and using larger sample volumes increased the detection limit from spiked whole blood to $50 \mathrm{CFU}$ per 
$\mathrm{ml}$ [42]. Our results are also very promising and showed that large volumes of whole blood need to be processed to ensure optimal detection rates. The pathogen enrichment strategies and DNA isolation procedures need to be further developed, integrated and automated to circumvent contamination risks and to decrease turnaround time. Furthermore, the pathogen enrichment strategies need to be investigated in well-defined clinical specimens with often low bacterial loads. Species-specific singleplex PCRs are not very useful for molecular diagnostics of BSI from whole blood, as BSI can be caused by a broad scala of pathogens. Clinical studies need to be conducted in which pathogen enrichment strategies are combined with broad-range (i.e. SepsiTest, Molzym) or multiplex PCRs (i.e MagicPlex Sepsis Test, Seegene) permitting an earlier administration of appropriate therapy. Nevertheless, it was demonstrated that sensitive pathogen detection and identification from whole blood was possible within three hours.

\section{DNA-based BSI diagnostics}

The use of PCR assays for detection and identification of pathogens causing BSI from whole blood, offers the fastest alternative as compared to blood cultures. Results can be available within a few hours as opposed to a few days. Moreover, it has been shown by others that prior antibiotic treatment does not negatively affect the performance of the PCR [43-45]. It has been reported that it is possible to decrease mortality with adequate treatment if sepsis is recognized in an early stage [46, 47].

In Chapter 8, one aspect was to investigate the ability of various biomarkers (C-reactive protein (CRP), procalcitonin (PCT), soluble urokinase plasminogen activator receptor (sUPAR), and neutrophil-lymphocyte count ratio (NLCR)) to predict BSI. PCT, sUPAR and NLCR were capable of differentiating patients with bacteremia from patients without in an emergency care unit (ECU). The discriminative power of PCT is in accordance with other reports which show that PCT is a more specific marker for BSI as compared to CRP [4850]. However, as PCT is an expensive biomarker, pre-screening of SIRS patients using PCT might be less cost-effective. Increased serum levels of suPAR positively correlate with the activation level of the immune system. However, suPAR levels are increased in relation to several conditions besides BSI [51, 52], i.e. cancer [53], HIV [54], malaria [55, 56], tuberculosis [57, 58], arthritis [59,60], and even liver fibrosis [61]. suPAR is a non-specific marker of inflammation, but higher suPAR levels are associated with increased mortality [62]. Although suPAR levels can be measured within 30 minutes, it is still an expensive and unspecific marker for BSI, rendering it unsuitable for pre-screening of suspected BSI patients. NLCR can be readily available when patients with SIRS present at the emergency department. De Jager et al. have described the NLCR in relation to predicting bacteremia at the emergency department [63]. NLCR was reported to have a higher prognostic accuracy as compared to other biomarkers. In our study, prediction of community-acquired bacteremia 
using a NLCR cut-off $\geq 10$ resulted in a sensitivity and negative predictive value of $85 \%$ and $94 \%$, respectively. NLCR is a cheap and easy to determine biomarker making it the most promising for future studies.

Also, the performance of two different commercially available molecular assays was examined and compared to blood culture results (Chapter 8). One $\mathrm{ml}$ residual whole blood of patients with suspected community-acquired BSI from the ECU was collected and used for selective pathogen DNA isolation with MolYsis (Molzym). DNA was analysed with SepsiTest (Molzym) and MagicPlex Sepsis Test (Seegene). When compared to blood culture results the performance of both tests was very poor (sensitivity, specificity, positive predictive value (PPV), and negative predictive value (NPV) for SepsiTest and MagicPlex Sepsis Test were $11 \%, 96 \%, 43 \%, 80 \%$, and $37 \%, 77 \%, 30 \%, 82 \%$, respectively). This poor performance might be due to several causes. Pathogen DNA should be obtained from larger volumes of whole blood, $5-10 \mathrm{ml}$, as we have shown that detection rates are higher as compared to using only 1 $\mathrm{ml}$ whole blood. This indicates that only prospective studies can be performed, because this required whole blood volume is not left after performing standard diagnostic tests. To enable optimal detection rates, it might be necessary, besides the use of larger blood volumes, to use the entire DNA sample (eluate) in PCR analysis. Furthermore, the performance of molecular assays should be studied in different well-defined patient populations. Most studies focus on the performance of molecular assays in critically ill patients from the ICU or even mixed patient populations in which better performances of molecular diagnostics have been found [44, 64-68]. Avolio et al. investigated 144 blood samples from patients presenting at the ECU with suspected sepsis [69]. In total 53 samples out of $144(37 \%)$ were positive by blood cultures or SeptiFAST PCR. In $30 / 53$ positive cases (56.6\%) both methods identified the same organisms. However, in $13 / 53$ cases (24.5\%) blood cultures identified pathogens not detected by PCR, and in 10 cases (18.9\%) SeptiFAST PCR gave positive results, whereas the blood cultures remained negative. Tsalik et al. also evaluated SeptiFAST PCR in 263 patient from the emergency department, and compared results to blood cultures. PCR identified an additional 24 organisms that blood culture failed to detect [70]. These results also support our findings and confirm that future studies are still a necessity to find explanations for discrepant results between blood cultures and PCR. Molecular diagnostics on whole blood facilitates the detection and identification of the etiologic agent of BSI within a few hours. Despite the discrepancies between blood cultures and PCR results, the first steps towards molecular pathogen diagnostics have been undertaken. Currently, blood cultures are still needed, but PCR allows more rapid initiation of pathogen-tailored antimicrobial therapy in patients.

\section{Role of RNA detection in BSI diagnostics}

Bacteremia is defined as the presence of viable bacteria in the bloodstream [71]. Detection of DNA by PCR does not provide information about the viability status of a pathogen as the DNA 
can originate from either living or dead pathogens [72-75]. Therefore, we attempted to find a marker that enabled us to measure $S$. aureus viability to be able to improve BSI diagnostics (Chapter 9). Our results clearly demonstrated that detection of $S$. aureus tuf mRNA correlated best with bacterial viability status (after antibiotic treatment) as determined by culture, while DNA (tuf and 16S rDNA) and rRNA (16S rRNA) detection could not be used to confirm viability. Cenciarini et al. showed that RNA targets differ greatly between bacterial species and different physiological conditions [76]. Future studies should attempt to find a panel of mRNA markers, which can be used to sensitively detect the most important BSI pathogens from whole blood, and indicate viability status at the same time. Additional (clinical) studies are required to confirm our preliminary findings.

\subsection{Conclusions and future perspectives}

All evaluated improvements for the laboratory diagnosis of BSI are displayed in bold black boxes in Figure 2. In summary, the results presented in this thesis clearly show the potential of both MALDI-TOF MS and PCR based methods for improved detection and identification, of pathogens causing BSI, from both blood cultures and subcultures (upper part in Figure 2). Implementation of these methods potentially reduce time-to-results, offer high sensitivity and specificity, and can improve the laboratory process for BSI, as previously discussed.

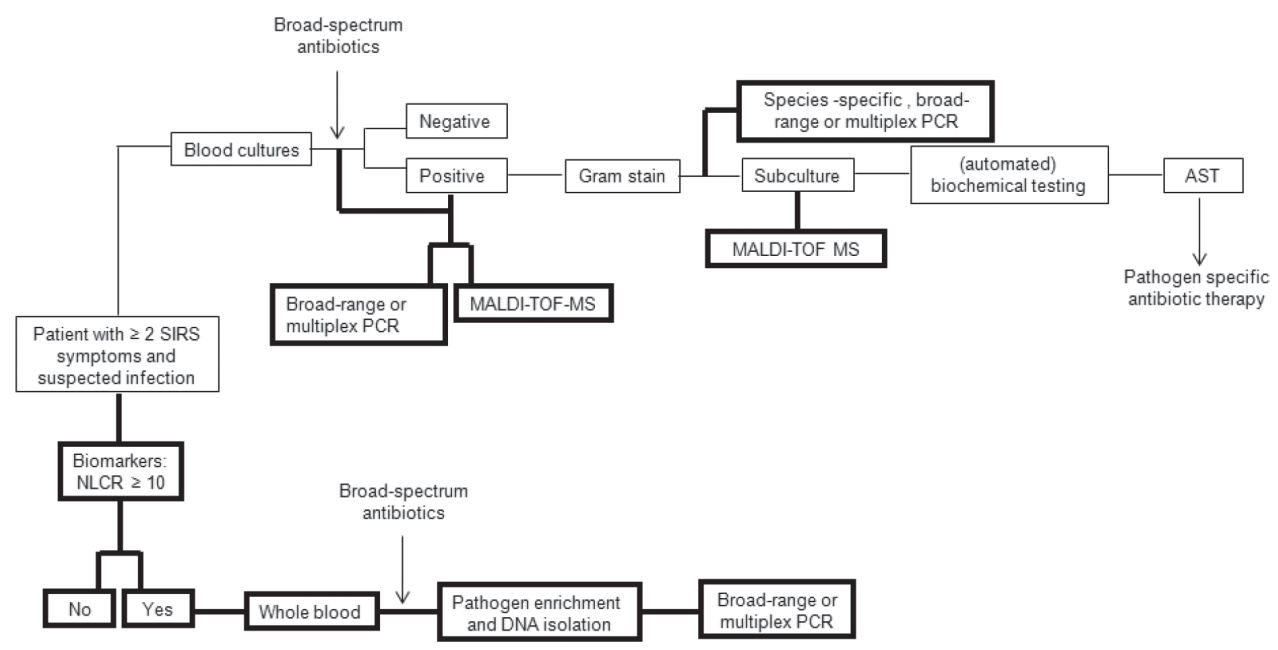

Figure 2. Overview of the evaluated improvements for the laboratory diagnosis of BSI. The bold black boxes represent the new approaches to decrease time-to-results for pathogen detection and identification from colonies (subculture), positive blood cultures and whole blood. SIRS; systemic inflammatory response syndrome, NLCR; neutrophil-lymphocyte count ratio, MALDI-TOF MS; matrix assisted laser desorption/ionisation- time of flight mass spectrometry, PCR; polymerase chain reaction, AST; antibiotic susceptibility testing. 
Direct pathogen detection and identification from whole blood is the only approach which currently enables time-to-results within a few hours (lower part in Figure 2). It has been shown that pathogen enrichment from $5 \mathrm{ml}$ whole blood results in a better detection rate as compared to $1 \mathrm{ml}$. Furthermore, BSI is caused by a broad scala of pathogens and the most frequently detected and most clinically relevant pathogens should be included in molecular assays. At present, AST is mainly determined by growth inhibition on solid media and this is very time-consuming as culture steps are still required. Future diagnostic assays should be able to process at least $5-10 \mathrm{ml}$ whole blood to ensure optimal detection rates. The amount of pathogens that can be identified should be extended, and complete antibiotic resistance screening should be included. Furthermore, our results seem to indicate that future assays should be able to screen for the presence of certain mRNA targets as these molecules seem to be better indicators for viable pathogens rather than the presence of DNA or rRNA, which will reflect dead pathogens as well. These different aspects should, ideally, be incorporated in one rapid and simple point of care diagnostic test which allows both pathogen DNA/RNA detection and AST, and is characterized by a "sample in, results out" principle. Results should be available as soon as possible and no special technical skills should be required. Currently, no commercially available molecular sepsis test for whole blood combines pathogen enrichment for large volumes of whole blood with rapid pathogen detection and identification. Seegene's MagicPlex Sepsis Test, when combined with MolYsis pathogen enrichment, approaches this ideal scenario the closest, but still takes approximately six hours and does not provide complete identification of the pathogens. To be able to create this future diagnostic assay, pathogen DNA enrichment and isolation should first be improved by reducing turnaround time. The commercially available MolYsis pathogen enrichment method takes at least two hours, depending on the number of samples to be processed. Pathogen enrichment combined with DNA isolation needs to be fully automated to reduce hands-ontime and contamination risks. Polaris (Biocartis) is currently being automated in a closed disposable cartridge. When available, this Polaris cartridge needs to be evaluated for $10 \mathrm{ml}$ whole blood, also in a clinical setting.

It will be difficult to incorporate complete AST in one assay, because antibiotic resistance is the result of a large number of genes and complex regulatory mechanisms. Microarray based assays have been developed to study the presence of large panels of antibiotic resistance genes $[77,78]$. However, microarray based tests often require special laboratory equipment and highly skilled personnel. Real-time PCR can be employed to detect clinically relevant antibiotic resistance genes from clinical samples (e.g. whole blood). Several commercially available sepsis test already offer detection of a small panel of antibiotic resistance genes (i.e. VYOO (SIRS-Lab), MagicPlex Sepsis Test (Seegene)). However, these available panels should be extended when more antibiotic resistance markers and virulence factors become 
available. Diagnostic tests that provide a complete antimicrobial susceptibility profile from whole blood are needed to be able to quickly initiate pathogen-specific antibiotic therapy. New developments are being introduced that might be a starting point for rapid pathogen identification and AST at the same time. An example is the next generation sequencing technology. Salipante et al. already demonstrated that next generation sequencing can be used to identify bacterial species in heterogeneous clinical specimens [79]. They used deep sequencing for a collection of sputum samples from cystic fibrosis patients and detected pathogens in specimens that were not positive by standard clinical culture methods, especially when fastidious bacteria were involved. Next generation sequencing technologies will, most likely, soon be able to provide high-resolution genotyping and will eventually be implemented in the field of clinical microbiology.

The current trends in molecular diagnostics for infectious diseases are moving towards automated, high-throughput technologies, such as next generation sequencing, and will provide an enormous amount of data. Bioinformaticians and/or company developed software packages are needed to guide us through these gigantic datasets.

As a result of the improvements presented in this thesis a significant reduction in turnaround time can be achieved for the laboratory diagnosis of BSI. Molecular diagnostic tests on large volumes of whole blood of BSI patients will, when optimised, improve patient care and reduce morbidity and mortality rates. However, future prospective studies are still required to prove our findings and determine if the whole blood approach is cost-effective. Whether either sensitive multiplex PCR assays or next generation sequencing, with pathogen enrichment, will be the basis for a simple point of care test in which rapid pathogen identification and AST from large volumes of whole blood can be combined, remains to be seen in the future. 


\section{References}

1. Bearman, G.M. and R.P. Wenzel, Bacteremias: a leading cause of death. Arch Med Res, 2005. 36(6): p. 646-59.

2. Esel, D., et al., Prospective evaluation of blood cultures in a Turkish university hospital: epidemiology, microbiology and patient outcome. Clin Microbiol Infect, 2003. 9(10): p. 103844.

3. Haug, J.B., et al., Bloodstream infections at a Norwegian university hospital, 1974-1979 and 1988-1989: changing etiology, clinical features, and outcome. Clin Infect Dis, 1994. 19(2): p. 246-56.

4. Martin, M.A., M.A. Pfaller, and R.P. Wenzel, Coagulase-negative staphylococcal bacteremia. Mortality and hospital stay. Ann Intern Med, 1989. 110(1): p. 9-16.

5. Ammerlaan, H.S., et al., Secular trends in nosocomial bloodstream infections: antibioticresistant bacteria increase the total burden of infection. Clin Infect Dis, 2013. 56(6): p. 798805.

6. de Kraker, M.E., et al., The changing epidemiology of bacteraemias in Europe: trends from the European Antimicrobial Resistance Surveillance System. Clin Microbiol Infect, 2012.

7. Klevens, R.M., et al., Estimating health care-associated infections and deaths in U.S. hospitals, 2002. Public Health Rep, 2007. 122(2): p. 160-6.

8. Stone, P.W., D. Braccia, and E. Larson, Systematic review of economic analyses of health care-associated infections. Am J Infect Control, 2005. 33(9): p. 501-9.

9. Kollef, M.H., Inadequate antimicrobial treatment: an important determinant of outcome for hospitalized patients. Clin Infect Dis, 2000. 31 Suppl 4: p. S131-8.

10. Kumar, A., et al., Initiation of inappropriate antimicrobial therapy results in a fivefold reduction of survival in human septic shock. Chest, 2009. 136(5): p. 1237-48.

11. Kumar, A., et al., Duration of hypotension before initiation of effective antimicrobial therapy is the critical determinant of survival in human septic shock. Crit Care Med, 2006. 34(6): p. 158996.

12. Wisplinghoff, H., et al., Nosocomial bloodstream infections in US hospitals: analysis of 24,179 cases from a prospective nationwide surveillance study. Clin Infect Dis, 2004. 39(3): p. 309-17.

13. Gebert, S., D. Siegel, and N. Wellinghausen, Rapid detection of pathogens in blood culture bottles by real-time PCR in conjunction with the pre-analytic tool MolYsis. J Infect, 2008. 57: p. 307-316.

14. Bos, M.M., et al., Bloodstream infections in patients with or without cancer in a large community hospital. Infection, 2013.

15. Hansen, W.L., et al., Molecular probes for diagnosis of clinically relevant bacterial infections in blood cultures. J Clin Microbiol, 2010. 48(12): p. 4432-8.

16. Tissari, P., et al., Accurate and rapid identification of bacterial species from positive blood cultures with a DNA-based microarray platform: an observational study. Lancet, 2010. 375(9710): p. 224-30.

17. Kloos, W.E. and K.H. Schleifer, Simplified scheme for routine identification of human Staphylococcus species. J Clin Microbiol, 1975. 1(1): p. 82-8.

18. Becker, K., et al., Development and evaluation of a quality-controlled ribosomal sequence database for $16 S$ ribosomal DNA-based identification of Staphylococcus species. J Clin Microbiol, 2004. 42(11): p. 4988-95.

19. Poyart, C., et al., Rapid and accurate species-level identification of coagulase-negative staphylococci by using the sodA gene as a target. J Clin Microbiol, 2001. 39(12): p. 4296-301.

20. Heikens, E., et al., Comparison of genotypic and phenotypic methods for species-level identification of clinical isolates of coagulase-negative staphylococci. J Clin Microbiol, 2005. 43(5): p. 2286-90.

21. Martineau, F., et al., Development of a PCR assay for identification of staphylococci at genus and species levels. J Clin Microbiol, 2001. 39(7): p. 2541-7.

22. Carbonnelle, E., et al., MALDI-TOF mass spectrometry tools for bacterial identification in clinical microbiology laboratory. Clin Biochem, 2011. 44(1): p. 104-9. 
23. van Veen, S.Q., E.C. Claas, and E.J. Kuijper, High-throughput identification of bacteria and yeast by matrix-assisted laser desorption ionization-time of flight mass spectrometry in conventional medical microbiology laboratories. J Clin Microbiol, 2010. 48(3): p. 900-7.

24. Dubois, D., et al., Identification of a variety of Staphylococcus species by MALDI-TOF mass spectrometry. J Clin Microbiol, 2010. 48: p. 941-945.

25. Spanu, T., et al., Evaluation of matrix-assisted laser desorption ionization-time-of-flight mass spectrometry in comparison to rpoB gene sequencing for species identification of bloodstream infection staphylococcal isolates. Clin Microbiol Infect, 2011. 17: p. 44-49.

26. Piette, A. and G. Verschraegen, Role of coagulase-negative staphylococci in human disease. Vet Microbiol, 2009. 134(1-2): p. 45-54.

27. von Eiff, C., G. Peters, and C. Heilmann, Pathogenesis of infections due to coagulasenegative staphylococci. Lancet Infect Dis, 2002. 2(11): p. 677-85.

28. Szabados, F., et al., The sensitivity of direct identification from positive BacT/ALERT (bioMerieux) blood culture bottles by matrix-assisted laser desorption ionization time-of-flight mass spectrometry is low. Clin Microbiol Infect, 2011. 17(2): p. 192-195.

29. Christner, M., et al., Rapid identification of bacteria from positive blood culture bottles by use of matrix-assisted laser desorption-ionization time of flight mass spectrometry fingerprinting. J Clin Microbiol, 2010. 48(5): p. 1584-91.

30. Drancourt, M., Detection of microorganisms in blood specimens using matrix-assisted laser desorption ionization time-of-flight mass spectrometry: a review. Clin Microbiol Infect, 2010. 16(11): p. 1620-5.

31. Ferreira, L., et al., Microorganisms Direct Identification from Blood Culture by Maldi-Tof Mass Spectrometry. Clin Microbiol Infect, 2011. 17(4): p. 546-551.

32. La Scola, B. and D. Raoult, Direct identification of bacteria in positive blood culture bottles by matrix-assisted laser desorption ionisation time-of-flight mass spectrometry. PLoS One, 2009. 4(11): p. e8041.

33. Moussaoui, W., et al., Matrix-assisted laser desorption ionization time-of-flight mass spectrometry identifies $90 \%$ of bacteria directly from blood culture vials. Clin Microbiol Infect, 2010. 16(11): p. 1631-8.

34. Prod'hom, G., et al., Matrix-assisted laser desorption ionization-time of flight mass spectrometry for direct bacterial identification from positive blood culture pellets. J Clin Microbiol, 2010. 48(4): p. 1481-3.

35. Stevenson, L.G., S.K. Drake, and P.R. Murray, Rapid Identification of Bacteria in Positive Blood Culture Broths by MALDI-TOF Mass Spectrometry. J Clin Microbiol, 2010. 48(2): p. 4447.

36. Edwards-Jones, V., et al., Rapid discrimination between methicillin-sensitive and methicillinresistant Staphylococcus aureus by intact cell mass spectrometry. J Med Microbiol, 2000. 49(3): p. 295-300.

37. Walker, J., et al., Intact cell mass spectrometry (ICMS) used to type methicillin-resistant Staphylococcus aureus: media effects and inter-laboratory reproducibility. J Microbiol Methods, 2002. 48(2-3): p. 117-26.

38. Yagupsky, P. and F.S. Nolte, Quantitative aspects of septicemia. Clin Microbiol Rev, 1990. 3(3): p. 269-79.

39. Harbarth, S., et al., Inappropriate initial antimicrobial therapy and its effect on survival in a clinical trial of immunomodulating therapy for severe sepsis. Am J Med, 2003. 115(7): p. 52935.

40. Engemann, J.J., et al., Adverse clinical and economic outcomes attributable to methicillin resistance among patients with Staphylococcus aureus surgical site infection. Clin Infect Dis, 2003. 36(5): p. 592-8.

41. Handschur, M., et al., Preanalytic removal of human DNA eliminates false signals in general $16 S$ rDNA PCR monitoring of bacterial pathogens in blood. Comp Immunol Microbiol Infect Dis, 2008 
42. Hansen, W.L., C.A. Bruggeman, and P.F. Wolffs, Evaluation of new preanalysis sample treatment tools and DNA isolation protocols to improve bacterial pathogen detection in whole blood. J Clin Microbiol, 2009. 47(8): p. 2629-31.

43. Corless, C.E., et al., Simultaneous detection of Neisseria meningitidis, Haemophilus influenzae, and Streptococcus pneumoniae in suspected cases of meningitis and septicemia using real-time PCR. J Clin Microbiol, 2001. 39(4): p. 1553-8.

44. Casalta, J.P., et al., Evaluation of the LightCycler(R) SeptiFast test in the rapid etiologic diagnostic of infectious endocarditis. Eur J Clin Microbiol Infect Dis, 2009. 28(6): p. 569-73.

45. Mauro, M.V., et al., Diagnostic utility of LightCycler SeptiFast and procalcitonin assays in the diagnosis of bloodstream infection in immunocompromised patients. Diagn Microbiol Infect Dis, 2012. 73(4): p. 308-11.

46. Dellinger, R.P., et al., Surviving Sepsis Campaign: international guidelines for management of severe sepsis and septic shock: 2008. Intensive Care Med, 2008. 34(1): p. 17-60.

47. Levy, M.M., et al., The Surviving Sepsis Campaign: results of an international guideline-based performance improvement program targeting severe sepsis. Intensive Care Med, 2010. 36(2): p. 222-31.

48. Chirouze, C., et al., Low serum procalcitonin level accurately predicts the absence of bacteremia in adult patients with acute fever. Clin Infect Dis, 2002. 35(2): p. 156-61.

49. Giamarellos-Bourboulis, E.J., et al., Assessment of procalcitonin as a diagnostic marker of underlying infection in patients with febrile neutropenia. Clin Infect Dis, 2001. 32(12): p. 171825.

50. Simon, L., et al., Serum procalcitonin and C-reactive protein levels as markers of bacterial infection: a systematic review and meta-analysis. Clin Infect Dis, 2004. 39(2): p. 206-17.

51. Hoenigl, M., et al., Diagnostic accuracy of soluble urokinase plasminogen activator receptor (sUPAR) for prediction of bacteremia in patients with systemic inflammatory response syndrome. Clin Biochem, 2013. 46(3): p. 225-9.

52. Molkanen, T., et al., Elevated soluble urokinase plasminogen activator receptor (suPAR) predicts mortality in Staphylococcus aureus bacteremia. Eur J Clin Microbiol Infect Dis, 2011. 30(11): p. 1417-24.

53. Sier, C.F., et al., The level of urokinase-type plasminogen activator receptor is increased in serum of ovarian cancer patients. Cancer Res, 1998. 58(9): p. 1843-9.

54. Ostrowski, S.R., et al., Soluble urokinase receptor levels in plasma during 5 years of highly active antiretroviral therapy in HIV-1-infected patients. J Acquir Immune Defic Syndr, 2004. 35(4): p. 337-42.

55. Ostrowski, S.R., et al., Plasma concentrations of soluble urokinase-type plasminogen activator receptor are increased in patients with malaria and are associated with a poor clinical or a fatal outcome. J Infect Dis, 2005. 191(8): p. 1331-41.

56. Perch, M., et al., Serum levels of soluble urokinase plasminogen activator receptor is associated with parasitemia in children with acute Plasmodium falciparum malaria infection. Parasite Immunol, 2004. 26(5): p. 207-11.

57. Eugen-Olsen, J., et al., The serum level of soluble urokinase receptor is elevated in tuberculosis patients and predicts mortality during treatment: a community study from GuineaBissau. Int J Tuberc Lung Dis, 2002. 6(8): p. 686-92.

58. Ostrowski, S.R., et al., Elevated levels of soluble urokinase receptor in serum from mycobacteria infected patients: still looking for a marker of treatment efficacy. Scand J Infect Dis, 2006. 38(11-12): p. 1028-32.

59. Pliyev, B.K. and M.Y. Menshikov, Release of the soluble urokinase-type plasminogen activator receptor (suPAR) by activated neutrophils in rheumatoid arthritis. Inflammation, 2010. 33(1): p. 1-9.

60. Slot, O., et al., Soluble urokinase plasminogen activator receptor in plasma of patients with inflammatory rheumatic disorders: increased concentrations in rheumatoid arthritis. Ann Rheum Dis, 1999. 58(8): p. 488-92. 
61. Andersen, E.S., et al., Twe/ve potential fibrosis markers to differentiate mild liver fibrosis from cirrhosis in patients infected with chronic hepatitis $C$ genotype 1. Eur J Clin Microbiol Infect Dis, 2011. 30(6): p. 761-6.

62. Koch, A., et al., Circulating soluble urokinase plasminogen activator receptor is stably elevated during the first week of treatment in the intensive care unit and predicts mortality in critically ill patients. Crit Care, 2011. 15(1): p. R63.

63. de Jager, C.P., et al., Lymphocytopenia and neutrophil-lymphocyte count ratio predict bacteremia better than conventional infection markers in an emergency care unit. Crit Care, 2010. 14(5): p. R192.

64. Dierkes, C., et al., Clinical impact of a commercially available multiplex PCR system for rapid detection of pathogens in patients with presumed sepsis. BMC Infect Dis, 2009. 9: p. 126.

65. Fitting, C., et al., DNAemia Detection by Multiplex PCR and Biomarkers for Infection in Systemic Inflammatory Response Syndrome Patients. PLoS One, 2012. 7(6): p. e38916.

66. Wellinghausen, N., et al., Diagnosis of bacteremia in whole-blood samples by use of a commercial universal 16S rRNA gene-based PCR and sequence analysis. J Clin Microbiol, 2009. 47(9): p. 2759-65.

67. Westh, H., et al., Multiplex real-time PCR and blood culture for identification of bloodstream pathogens in patients with suspected sepsis. Clin Microbiol Infect, 2009. 15(6): p. 544-51.

68. Yanagihara, K., et al., Evaluation of pathogen detection from clinical samples by real-time polymerase chain reaction using a sepsis pathogen DNA detection kit. Crit Care, 2010. 14(4): p. R159.

69. Avolio, M., et al., Molecular identification of bloodstream pathogens in patients presenting to the emergency department with suspected sepsis. Shock, 2010. 34(1): p. 27-30.

70. Tsalik, E.L., et al., Multiplex PCR to diagnose bloodstream infections in patients admitted from the emergency department with sepsis. J Clin Microbiol, 2010. 48(1): p. 26-33.

71. M, S., in Stedman's Medical Dictionary1995, Md: Lippincott Williams \& Wilkins: Baltimore.

72. Hellyer, T.J., et al., Detection of viable Mycobacterium tuberculosis by reverse transcriptasestrand displacement amplification of mRNA. J Clin Microbiol, 1999. 37(3): p. 518-23.

73. Iyer, R., et al., Detection of Borrelia burgdorferi Nucleic Acids after Antibiotic Treatment Does Not Confirm Viability. J Clin Microbiol, 2013. 51(3): p. 857-62.

74. Josephson, K.L., C.P. Gerba, and I.L. Pepper, Polymerase chain reaction detection of nonviable bacterial pathogens. Appl Environ Microbiol, 1993. 59(10): p. 3513-5.

75. Morre, S.A., et al., Monitoring of Chlamydia trachomatis infections after antibiotic treatment using RNA detection by nucleic acid sequence based amplification. Mol Pathol, 1998. 51(3): p. 149-54.

76. Cenciarini, C., et al., Influence of long time storage in mineral water on RNA stability of Pseudomonas aeruginosa and Escherichia coli after heat inactivation. PLoS One, 2008. 3(10): p. e3443.

77. Call, D.R., et al., Identifying antimicrobial resistance genes with DNA microarrays. Antimicrob Agents Chemother, 2003. 47(10): p. 3290-5.

78. Perreten, V., et al., Microarray-based detection of 90 antibiotic resistance genes of grampositive bacteria. J Clin Microbiol, 2005. 43(5): p. 2291-302.

79. Salipante, S.J., et al., Rapid 16S rRNA Next-Generation Sequencing of Polymicrobial Clinical Samples for Diagnosis of Complex Bacterial Infections. PLoS One, 2013. 8(5): p. e65226. 



\section{Chapter 11}

Samenvatting, Discussie en Toekomstvisies 
Het aantal patiënten met een bloedbaaninfectie (BBI) neemt nog steeds toe. BBIs kenmerken zich door hoge mortaliteit en toenemende zorgkosten. Optimale behandeling van een BBI is afhankelijk van een aantal factoren, zoals het herkennen van de BBI, het uitvoeren van laboratoriumtesten en het initiëren van een correcte (pathogeen specifieke) behandeling. Om een patiënt met een pathogeen specifiek antibioticum te kunnen behandelen, is het van belang het micro-organisme (pathogeen) welke de BBI veroorzaakt snel te detecteren en te identificeren. Wanneer een BBI tot een septische shock leidt, is tijd van cruciaal belang. Als bij een ernstige sepsis niet binnen het uur snel en adequaat behandeld wordt, daalt de overlevingskans van een patiënt van $80 \%$ naar minder dan $10 \%$, wanneer adequate behandeling pas wordt gestart na 24 uur. Tevens zal het toedienen van een pathogeen specifiek antibioticum het ontstaan van resistente stammen afremmen.

De "gouden standaard" voor detectie en identificatie van pathogenen die BBI veroorzaken bestaat nog steeds uit kweekmethoden. Wanneer bloedkweken positief worden bevonden door het geautomatiseerde kweeksysteem, zal vervolgens een Gramkleuring worden uitgevoerd. Dit wordt dan gevolgd door het inzetten van een subkweek op verschillende voedingsbodems. De kolonies die ontstaan op de voedingsbodems, kunnen worden gebruikt voor (geautomatiseerde) biochemische testen. De resultaten van die biochemische testen zorgen in de meeste gevallen voor pathogeen identificatie. Tot slot zullen ook antibiotica gevoeligheidstesten worden ingezet, waarna de patiënt van breedspectrum antibiotica op een pathogeen specifieke behandeling kan worden overgezet (Figuur 1).

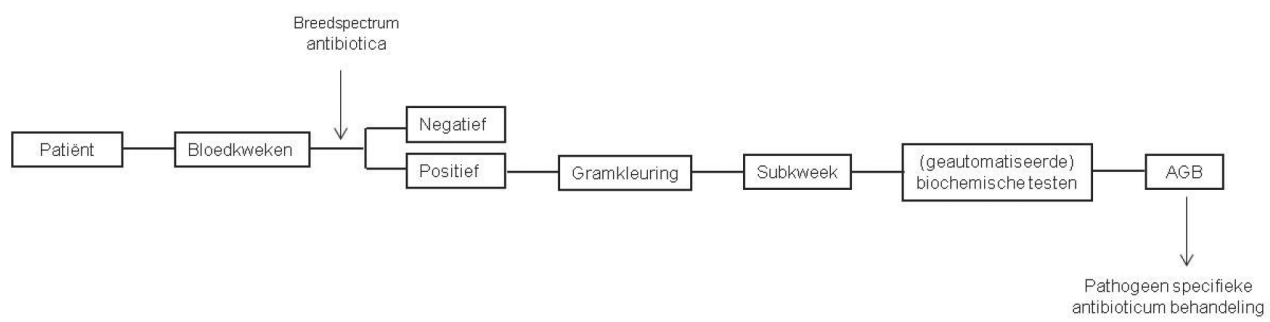

Figuur 1. Het conventionele diagnostiek proces voor bloedbaaninfecties.

Wanneer bloedkweken positief worden bevonden door het geautomatiseerde kweeksysteem, zal een Gramkleuring worden uitgevoerd. Vervolgens zal er een subkweek worden ingezet op verschillende voedingsbodems, waarna (geautomatiseerde) biochemische testen worden ingezet voor pathogeen identificatie. Een volledige antibiotica gevoeligheidsbepaling bestaat vaak uit het onderzoeken van de mate van remming, op de groei van bacteriën in aanwezigheid van verschillende antibiotica. AGB; antibiotica gevoeligheidsbepaling.

Dit conventionele proces neemt vaak minimaal een paar dagen in beslag. Daarnaast is bekend dat bloedkweken, die micro-organismen met complexe voedingseisen, of micro-organismen welke langzaam groeien en/of bloed van patiënten bevatten die al antibioticum behandeling 
ondergaan, vals negatief kunnen blijven. Het belangrijkste doel van dit onderzoek was het bestuderen van verschillende strategieën, om zo het bestaande laboratoriumproces voor een $\mathrm{BBI}$ te verbeteren (Figuur 1). Deze strategieën omvatten eiwit gebaseerde methoden, zoals MALDI-TOF massa spectrometrie, maar ook methoden voor het aantonen van nucleïnezuren (DNA/RNA), zoals de polymerase ketting reactie (PCR). Deze technologieën zijn toegepast op subkweken (kolonies op een voedingsbodem), maar ook op (positieve) bloedkweken en volbloed (direct uit de patiënt), om zo de tijd tot resultaten te verkorten. Daarnaast is ook aandacht besteedt aan monstervoorbereiding voor de verschillende klinische materialen.

\subsection{BBI diagnostiek uit bloedkweken en subkweken}

\section{Polymerase ketting reactie (PCR)}

Grampositieve bacteriën (bijvoorbeeld Stafylococcus aureus, coagulase-negatieve stafylococcen (CoNS) en Enterococcus spp.), worden vaak aangetoond in bloedkweken. We hebben daarom 2 real-time PCR duplex testen ontwikkeld voor detectie van S. aureus, CoNS en Enterococcus spp. Deze testen zijn klinisch geëvalueerd door gebruik te maken van: 1) positieve bloedkweken met Grampositieve kokken en 2) $1 \mathrm{ml}$ restant volbloed van intensive care unit (ICU) patiënten met een BBI verdenking (Hoofdstuk 2). De duplex testen, gebaseerd op het tuf gen, maakten het mogelijk om snel en accuraat pathogenen te identificeren vanuit positieve bloedkweken. In een kleine studie waarin $1 \mathrm{ml}$ restbloed werd gebruikt van ICU patiënten met een BBI verdenking, werden de ontwikkelde PCR testen gecombineerd met een gevoelige bacterieel DNA isolatie methode. In 18/22 patiënten (82\%) werden identieke resultaten verkregen tussen bloedkweek en PCR.

Tegelijkertijd hebben we onderzocht of het mogelijk was om pathogenen te identificeren uit bloedkweken, die nog niet positief waren bevonden door het automatische kweeksysteem

(Hoofdstuk 3). ZesverschillendepathogeenDNAisolatiemethoden voorbloedkweekmateriaal werden vergeleken, om zo de beste methode te vinden voor DNA isolatie uit bloedkweken. De beste methode werd gebruikt om pathogeen DNA te isoleren uit bloedkweek flessen, welke maar enkele uren in het kweeksysteem hadden gestaan. Op dit geïsoleerde DNA werd de ontwikkelde PCR ingezet. De MolYsis Plus methode (Molzym GmbH, Bremen, Duitsland) gecombineerd met het specifiek B programma op de EasyMAG resulteerde in de beste isolatie van bacterieel DNA uit BacT/ALERT bloedkweek flessen (bioMérieux, Marcy L'Etoile, Frankrijk). Reconstructie experimenten met $S$. aureus resulteerde in een detectielimiet van 10 kolonie vormende eenheden (CFU)/ml volbloed met de ontwikkelde tuf gebaseerde PCR. BACTEC (BD) bloedkweek flessen resulteerde in een 1 log lagere detectielimiet. Na een incubatiestap van de BacT/ALERT bloedkweek flessen van 5 uur, konden zowel S. aureus als CoNS worden aangetoond, dit door gebruik te maken van de real-time PCR testen. 
Door deze verkorte incubatiestap toe te passen, konden $S$. aureus en CoNS in 1 werkdag geïdentificeerd worden. In vergelijking met het conventionele diagnostiek proces, waren de resultaten 19 uur eerder beschikbaar. Gebert et al. hebben vergelijkbare resultaten laten zien. Zij hebben ook reconstructie experimenten uitgevoerd en een reductie van 5.0-8.7 uur bereikt, echter, in klinische monsters lieten ze zelfs een gemiddelde reductie in tijd tot pathogeen identificatie van 10.7 uur zien. Laboratoria die 24-uur per dag actief zijn zouden de meeste winst kunnen behalen met deze strategie. Meer onderzoek is nodig, om vast te stellen welke incubatietijd geschikt is voor de meest voorkomende pathogenen. De tuf realtime PCR is enkel inzetbaar voor patiënten met een verdenking op een BBI veroorzaakt door Stafylococcus spp. of Enterococcus spp. BBIs kunnen echter veroorzaakt worden door een breed scala aan pathogenen en dit maakt soortspecifieke PCRs minder waardevol, wanneer er een optimale tijd tot resultaten nodig is. De ontwikkelde real-time PCR kan aangepast worden, zodat de meest voorkomende BBI pathogenen aangetoond kunnen worden uit bloedkweken. Deze flexibiliteit zorgt ervoor, dat de test aangepast kan worden aan de lokale epidemiologie, of een specifieke patiëntengroep. Toekomstige studies zouden ook gebruik kunnen maken van de multi-probe test, zoals beschreven door Hansen et al., of de commercieel verkrijgbare Prove-it Sepsis test (MobiDiag, Helsinki, Finland). Deze testen zijn echter ontwikkeld voor pathogeen identificatie uit positieve bloedkweken. Het zal moeten blijken of deze testen gevoelig genoeg zijn, om gebruikt te kunnen worden op bloedkweken, die nog niet positief zijn bevonden door het automatische kweeksysteem.

\section{MALDI-TOF MS}

Infecties door Grampositieve bacteriën kenmerken zich door hoge morbiditeit en mortaliteit. Een Amerikaanse studie heeft aangetoond, dat in patiënten met een BBI vaak Grampositieve bacteriën worden gevonden. De meest geïsoleerde groep van pathogenen uit bloedkweken waren CoNS (31\%). CoNS komen voor op de huid en de slijmvliezen van de mens. Ze zijn lange tijd beschouwd als bron van besmetting van bloedkweken, maar omdat ze steeds meer worden gekweekt hebben de CoNS meer aandacht gekregen en wordt hun rol als pathogeen beter onderzocht. Snelle en betrouwbare determinatie van CoNS op species niveau zou daarom klinisch relevant kunnen zijn. Fenotypische methoden (biochemische testen) volgens Kloos en Schleifer zijn lange tijd de gouden standaard geweest voor identificatie van CoNS op species niveau. De API 32 Staph strip (bioMérieux) en de geautomatiseerde VITEK 2 (bioMérieux) hebben de fenotypische manier van identificeren verbeterd. Een aantal genen, zoals $16 S$ rRNA, sodA en tuf, zijn geschikt voor genotypische identificatie van CoNS. Analyse van eiwitprofielen van micro-organismen is mogelijk met Matrix Assisted Laser Desorption Ionisation-Time, of Flight massa spectrometrie (MALDI-TOF MS). Deze nieuwe techniek is geschikt om snel en accuraat micro-organismen te identificeren. We hebben 5 methoden vergeleken op dezelfde CoNS stammen, om zo de beste methode te vinden voor CoNS 
identificatie: 1) VITEK 2, 2) ID 32 Staph strip, 3) 16S rDNA gen sequencing (MicroSeq, Applied Biosystems), 4) tuf gen sequencing en 5) MALDI-TOF MS (Bruker Daltonics) (Hoofdstuk 4). De verkregen resultaten toonden aan, dat MALDI-TOF MS de beste methode was voor CoNS species identificatie, $99.3 \%$ van de CoNS stammen werden correct geïdentificeerd. Sequensen van het tuf gen is het meest geschikte alternatief. Heikens et al. hebben ook aangetoond, dat tuf gen sequencing een goede manier is, om CoNS te identificeren (5/57 correct geïdentificeerd). Wanneer snelle en accurate identificatie van CoNS nodig is, blijkt MALDI-TOF MS de meest geschikte methode. Dit is ook door anderen aangetoond.

Het blijft een uitdaging in de dagelijkse praktijk, om klinisch relevante CoNS te onderscheiden van CoNS stammen die de bloedkweek fles hebben besmet. De meest optimale methode voor CoNS identificatie was MALDI-TOF MS. Deze technologie werd ingezet om de klinische relevantie van het identificeren van CoNS op species niveau in kaart te brengen (Hoofdstuk 5). De meeste CoNS infecties zijn het gevolg van het gebruik van medische hulpmiddelen, zoals protheses en (intraveneuze) catheters. S. lugdunensis en S. schleiferi worden echter ook gevonden als veroorzaker van $\mathrm{BBI}$ en endocarditis en $S$. saprofyticus als veroorzaker van urineweginfecties. We hebben aangetoond, dat identificatie van CoNS op species niveau kan bijdragen aan het vaststellen, of een patiënt daadwerkelijk lijdt aan een CoNS BBI. De CoNS species die het meest gevonden werd bij BBIs was S. epidermidis, maar deze CoNS soort was tevens ook de meest gevonden bloedkweekfles besmetting. Daarnaast hebben we aangetoond, dat $S$. haemolyticus het meest geassocieerd werd met $\mathrm{BBI}$ en de meeste andere CoNS soorten toch vaak als besmetting van de kweekfles konden worden bestempeld. Dit betekent dat CoNS identificatie van klinisch belang kan zijn, om zo een pathogeen van potentiële besmetting te kunnen onderscheiden. CoNS identificatie gecombineerd met het medisch inzicht van de arts-microbioloog, zou kunnen leiden tot een optimalere patiëntenzorg. Moleculaire diagnostische testen zouden alle klinisch relevante CoNS soorten moeten kunnen detecteren. Vervolgstudies zijn nodig om onze bevindingen te staven.

Snellere identificatie van micro-organismen direct uit positieve bloedkweken, kan grote impact hebben in de kliniek. Om die reden hebben we onderzocht, of het mogelijk was om bacteriën direct te identificeren uit positieve aerobe BacT/ALERT bloedkweken met behulp van MALDI-TOF MS. Daarnaast is geëvalueerd of verkorte incubatie (7 uur) van S. aureus en Escherchia coli bevattende BacT/ALERT bloedkweekflessen, gecombineerd met identificatie door middel van MALDI-TOF MS, mogelijk was (Hoofdstuk 6). Wanneer bloedkweken worden gebruikt als startmateriaal voor MALDI-TOF MS zullen de humane cellen moeten worden verwijderd, om storing van humane eiwitten in de analyse te voorkomen. Daarom zijn verschillende methoden geanalyseerd en is onderzocht welke methode het meest geschikt is voor het verwijderen van deze aspecifieke eiwitten. Uit de resultaten bleek dat bacteriële identificatie mogelijk is binnen 20 minuten uit positieve bloedkweken, wanneer er 
gebruik wordt gemaakt van SepsiTyper (Bruker Daltonics). Dit is natuurlijk ook afhankelijk van de te verwerken hoeveelheid bloedkweken. Het conventionele diagnostiekproces neemt ongeveer 1-2 dagen in beslag. In de reconstructie experimenten heeft verkorte incubatie (7 uur) van aerobe $S$. aureus, of E. coli bevattende BacT/ALERT bloedkweken geen betrouwbare identificatie opgeleverd. Een alternatief, voor het gebruik van positieve bloedkweken in combinatie met MALDI-TOF MS pathogeen identificatie, is het gebruiken van spot-subkweken op voedingsbodems. Deze spot-subkweek bestaat uit het kweken van positief bloedkweek materiaal gedurende ongeveer 4 uur op een voedingsbodem. Over het algemeen zijn er na 4 uur genoeg micro-organismen op de voedingsbodem aanwezig, welke gebruikt kunnen worden voor MALDI-TOF MS. Bloedkweken die 's ochtends positief worden, zouden via deze spot-subkweek geanalyseerd kunnen worden en bloedkweken die later op de werkdag positief worden kunnen direct worden gebruikt (m.b.v. SepsiTyper). Szabados et al. beschreef een lage sensitiviteit (30\%) wanneer positieve BacT/ALERT bloedkweek flessen werden gebruikt voor MALDI. Anderen toonden aan dat MALDI-TOF MS zeer geschikt is voor pathogeen identificatie uit positieve bloedkweken, zowel BacT/ALERT als BACTEC flessen. MALDI-TOF MS kan zorgen voor een snellere tijd tot identificatie, maar de hoeveelheid pathogenen aanwezig in de bloedkweekfles zijn daarbij van cruciaal belang. Er moeten minimaal $10^{7}-10^{8} \mathrm{CFU} / \mathrm{ml}$ aanwezig zijn, om een goed eiwitprofiel te verkrijgen. Dit betekent dat kweekstappen nog noodzakelijk zijn om resultaten te kunnen genereren. Het is tevens nog niet mogelijk om MALDI-TOF MS te gebruiken voor antibiotica gevoeligheidsbepalingen (AGB). De eerste stappen richting AGB met MALDI-TOF MS zijn gezet, maar het is nog niet mogelijk een compleet antibiogram te verkrijgen.

\subsection{BBI diagnostiek direct uit volbloed}

\section{Monstervoorbereiding}

Pathogeen detectie en identificatie direct uit volbloed is de enige manier om kweekstappen te omzeilen. Monstervoorbereiding is dan van cruciaal belang om humane cellen en DNA te kunnen verwijderen, en tegelijkertijd pathogenen te verrijken, om zo de kleine hoeveelheid bacteriën aan te kunnen tonen.

Detectie van pathogeen DNA uit volbloed is een veelbelovende strategie, om snel een BBI te kunnen diagnosticeren en dus snel pathogeen specifieke therapie te kunnen starten. Dit is heel belangrijk omdat bekend is, dat het gebruik van een onjuist antibioticum kan resulteren in toenemende mortaliteit. Daarnaast is beschreven dat gebruik van een inadequaat antibioticum en fout gebruik van een antibioticum, leiden tot de ontwikkeling van resistente stammen, wat weer schadelijk is voor de patiënt. Om gelijkwaardige gevoeligheden te bereiken als met de bloedkweken zijn verschillende pathogeen DNA verrijkingmethoden onderzocht 
(Hoofdstuk 7). Reconstructie-experimenten met S. aureus, Pseudomonas aeruginosa en Candida albicans hebben aangetoond, dat pathogeen verrijking en DNA isolatie uit $5 \mathrm{ml}$ volbloed, met zowel Polaris (Biocartis) als MolYsis (Molzym), gevolgd door soortspecifieke real-time PCRs betrouwbare en gevoelige detectie tot gevolg had. De EasyMAG kan maar $200 \mu \mathrm{l}$ volbloed verwerken en daarom waren de detectiepercentages met deze methode teleurstellend. Polaris was de meest gevoelige methode (hoogste detectiepercentages, $100 \%$ score voor de monsters met 10 CFU/ml). Resultaten waren binnen 3 uur beschikbaar. In onze studie hebben we laten zien, dat zowel Polaris, als MolYsis voldoende humane achtergrond verwijderde. Er is geen storing geobserveerd in de soortspecifieke PCRs. Anderen hebben ook laten zien, dat de selectieve lysis van bloedcellen en (niet)-enzymatische degradatie van humaan DNA een meer betrouwbare detectie van pathogeen DNA tot gevolg heeft. Handschur et al. toonden in een 16S rDNA PCR, dat wanneer ze gebruik maakten van DNA monsters waar de humane achtergrond niet was verwijderd, ze veel aspecifieke signalen oppikten door de aanwezigheid van humaan DNA. Daarom hebben ze een verrijkingsmethode ontwikkeld voor de analyse van bloedmonsters. Klinische monsters (volbloed) lieten vergelijkbare resultaten zien, wanneer ze die vergeleken met de bloedkweek uitslagen. De ontwikkelde verrijkingsmethode zorgde voor specifieke en gevoelige detectie van pathogenen in PCR testen. Hansen et al. hebben aangetoond, dat een betere detectielimiet (50 CFU/ $\mathrm{ml}$ ) kan worden verkregen, wanneer een verrijkingsmethode en het gebruik van grotere volumes volbloed werden gecombineerd. De resultaten van onze studie zijn veelbelovend en hebben duidelijk laten zien, dat grote volumes volbloed moeten worden gebruikt om goede detectiepercentages te bewerkstelligen. De pathogeen verrijkingsmethoden en DNA isolatie procedures moeten verder worden ontwikkeld, geïntegreerd en geautomatiseerd, dit om sneller resultaat te verkrijgen en besmettingsrisico's te verlagen. Daarnaast moeten deze methoden worden onderzocht in goed gedefinieerde klinische monsters, waar vaak lage hoeveelheden pathogenen aanwezig zijn. Soortspecifieke PCRs zijn niet nuttig voor moleculaire diagnostiek van BBls uit volbloed, omdat BBls kunnen worden veroorzaakt door vele verschillende micro-organismen. Er moeten klinische studies worden uitgevoerd waarin pathogeen verrijkingsmethoden worden gecombineerd met breed dekkende (SepsiTest, Molzym), of multiplex PCRs (MagicPlex Sepsis Test, Seegene). Enkel op die manier kan er sneller doelgericht behandeld worden. Niettemin zijn we momenteel in staat, om binnen drie uur pathogenen te detecteren en identificeren uit volbloed.

\section{DNA gebaseerde diagnostiek voor BBI}

In vergelijking met bloedkweken bieden PCR testen voor detectie en identificatie van BBI veroorzakende pathogenen het snelste alternatief. Resultaten kunnen binnen enkele uren beschikbaar zijn, in plaats van na een paar dagen. Bovendien beïnvloed voorafgaand antibioticum gebruik de PCR niet. Men heeft aangetoond dat het mogelijk is, om mortaliteit 
te verlagen met adequate antibioticum therapie, wanneer sepsis snel wordt herkend. In Hoofdstuk 8 is onderzocht, of er een biomarker (C-reactive protein (CRP), procalcitonine (PCT), soluble urokinase plasminogen activator receptor (suPAR) en neutrofiel-lymfocyt count ratio (NLCR)) in staat is $B B I$ te voorspellen in patiënten, die behandeld worden op de eerste hulp. PCT, suPAR en NLCR kunnen allen ingezet worden om patiënten met en zonder bacteriëmie te onderscheiden. Het onderscheidende vermogen van PCT komt overeen met de literatuur, waarin ook bevestigd wordt dat PCT een specifiekere biomarker is voor $\mathrm{BBI}$ dan CRP. PCT is echter wel een dure biomarker bepaling en pre-screening van SIRS patiënten met PCT, zou daarom minder kosteneffectief kunnen zijn. Hoge serum waarden van suPAR correleren met verschillende aandoeningen naast BBI, bijvoorbeeld kanker, HIV, malaria, tuberculose, artritis en zelfs leverfibrose. suPAR is een aspecifieke marker voor ontsteking, maar hoge suPAR waarden zijn geassocieerd met hogere kans op overlijden. suPAR waarden kunnen binnen 30 minuten beschikbaar zijn, maar omdat het een dure en aspecifieke marker voor $\mathrm{BBI}$ is, is deze biomarker ongeschikt voor pre-screening van $B B I$ verdachte patiënten. NLCR is snel beschikbaar nadat patiënten zich melden bij de eerste hulp (met SIRS symptomen en infectie verdenking). De Jager et al. hebben onderzoek gedaan naar NLCR in relatie tot het voorspellen van bacteriëmie op de eerste hulp en deze biomarker kwam als beste uit de studie. Onze studie heeft aangetoond dat het voorspellen van bacteriëmie, met NLCR waarden $\geq 10$, resulteert in een sensitiviteit en negatief voorspellende waarde van respectievelijk $85 \%$ en $94 \%$. NLCR is een goedkope en simpel te bepalen biomarker en daarom het meest geschikt voor vervolg studies.

Ook zijn twee verschillende commercieel verkrijgbare moleculaire testen geëvalueerd, en de resultaten zijn vergeleken met bloedkweek uitslagen van dezelfde patiënt (Hoofdstuk 8). Eén $\mathrm{ml}$ restant volbloed van eerste hulp patiënten met een BBI verdenking is verzameld en gebruikt voor selectieve pathogeen DNA isolatie met MolYsis (Molzym). Het verkregen DNA werd geanalyseerd met SepsiTest (Molzym) en MagicPlex Sepsis Test (Seegene). De resultaten werden vergeleken met de uitslagen van de bloedkweken en waren teleurstellend (sensitiviteit, specificiteit, positief voorspellende waarde en negatief voorspellende waarde voor SepsiTest en MagicPlex Sepsis Test waren respectievelijk 11\%, 96\%, 43\%, 80\% en $37 \%, 77 \%, 30 \%, 82 \%)$. Er zijn verschillende verklaringen voor deze resultaten. Pathogeen DNA zou moeten worden geïsoleerd uit grotere volumes volbloed, namelijk 5-10 ml. We hebben eerder laten zien dat detectiepercentages hoger zijn, wanneer meer dan $1 \mathrm{ml}$ bloed wordt gebruikt. Dit betekent dat enkel prospectieve studies kunnen worden uitgevoerd, omdat er niet voldoende restbloed over is na het uitvoeren van standaard diagnostiek testen. Om optimale detectiepercentages te kunnen garanderen, zou naast het gebruik van een hoger volume bloed, ook het volledige DNA monster gebruikt moeten worden voor PCR analyse. Bovendien moeten de prestaties van moleculaire testen bestudeerd worden in verschillende goed gedefinieerde patiëntengroepen. De meeste studies onderzoeken de 
mogelijkheden van moleculaire testen in de meest kritisch zieke patiënten, bijvoorbeeld van de ICU en soms wordt zelfs een mix van patiëntengroepen gebruikt. In die studies, presteren de moleculaire testen vaak wel goed. Avolio et al. heeft 144 bloedmonsters van eerste hulp patiënten onderzocht en in totaal waren er 53 positief $(37 \%)$ met bloedkweek en SeptiFAST (Roche). In 30/53 gevallen hebben de bloedkweken pathogenen opgeleverd, die niet met PCR aangetoond waren en in 10 gevallen leidde SeptiFAST PCR tot positieve resultaten, die niet bevestigd werden met kweek. Tsalik et al. heeft ook de SeptiFAST PCR geëvalueerd en gebruikte daarvoor bloedmonsters van 263 eerste hulp patiënten. Ook in deze studie werden de PCR resultaten vergeleken met bloedkweek uitslagen. PCR heeft 24 extra positieve monsters opgeleverd, welke niet zijn opgepikt met bloedkweek. De resultaten van Avolio en Tsalik ondersteunen de gegevens uit ons onderzoek en bevestigen dat meer onderzoek nodig is, om een verklaring te kunnen geven voor de discrepanties tussen PCR en kweek. Moleculaire diagnostiek op volbloed faciliteert de detectie en identificatie van het pathogeen welke de BBI veroorzaakt binnen enkele uren. Ondanks de discrepanties tussen PCR en bloedkweken kunnen we stellen, dat de eerste stappen naar moleculaire detectie van pathogenen gezet zijn. Momenteel blijven bloedkweken nog noodzakelijk, maar PCR zou ervoor kunnen zorgen, dat patiënten sneller pathogeen specifieke antibioticum behandeling kunnen krijgen.

\section{De rol van RNA detectie in BBI diagnostiek}

Bacteriëmie is gedefinieerd, als de aanwezigheid van levende bacteriën in de bloedbaan. Detectie van pathogeen DNA met behulp van PCR geeft echter geen informatie over de levensvatbaarheid van een pathogeen. Dit DNA kan namelijk afkomstig zijn van zowel dode, als levende pathogenen. We hebben daarom getracht een marker te vinden die ons in staat stelt om de levensvatbaarheid van $S$. aureus te meten, om zo de BBI diagnostiek te verbeteren (Hoofdstuk 9). Onze resultaten lieten duidelijk zien, dat de detectie van $S$. aureus tuf mRNA het best correleerde met de bacteriële levensvatbaarheid (na antibioticum behandeling), zoals gedemonstreerd met kweek. DNA (Tuf en 16S rDNA) en rRNA (16S rRNA) konden niet worden gebruikt, om de levensvatbaarheid te onderzoeken.

Cenciarini et al. hebben aangetoond, dat verscheidene RNA transcripten erg variëren tussen verschillende bacteriesoorten en fysiologische omstandigheden. Toekomstige studies zouden moeten trachten een mRNA panel samen te stellen welke gebruikt kan worden, om zo gevoelig mogelijk de meest belangrijke BBI pathogenen aan te tonen uit volbloed en daarnaast kan aantonen of de micro-organismen nog levensvatbaar zijn. Er zijn meer (klinische) studies nodig om onze voorlopige resultaten te bevestigen. 


\subsection{Conclusies en toekomstvisies}

Alle geëvalueerde methoden voor verbetering van het diagnostiekproces voor BBI zijn weergegeven in de dikke zwarte boxen in Figuur 2. Samenvattend, de gepresenteerde resultaten in dit proefschrift hebben duidelijk de potentie van zowel MALDI-TOF MS en PCR gebaseerde methoden laten zien. Beide strategieën leiden tot verbeterde detectie en identificatie van pathogenen, die BBls veroorzaken uit zowel bloedkweken, als subkweken (bovenste deel Figuur 2). Implementatie van deze methoden kan leiden tot verkorte tijd tot resultaten, ze bieden hoge sensitiviteit en specificiteit. Tevens kunnen deze methoden het laboratoriumproces van BBI verbeteren.

Momenteel is directe detectie en identificatie van pathogenen uit volbloed de enige benadering, die het mogelijk maakt binnen een paar uur resultaten te genereren (onderste deel Figuur 2). Pathogeen verrijking uit $5 \mathrm{ml}$ bloed resulteert in een beter detectiepercentage in vergelijking tot $1 \mathrm{ml}$ bloed. Omdat BBls het gevolg kunnen zijn van een breed scala aan pathogenen, zou een moleculaire test de meest voorkomende en de meest klinische relevante moeten kunnen aantonen. Momenteel wordt de AGB nog steeds uitgevoerd door middel van het meten van groeiremming op voedingsbodems. Aangezien hier kweekstappen voor nodig

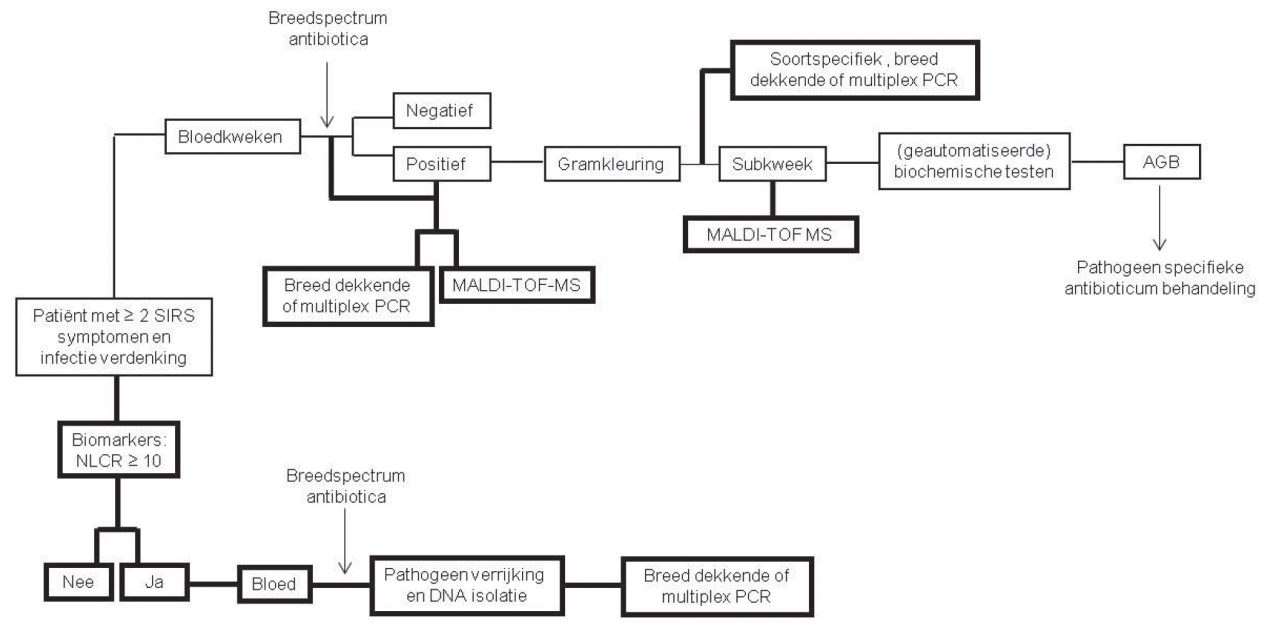

Figuur 2. Overzicht van de geëvalueerde stappen voor verbetering van de laboratoriumdiagnostiek voor bloedbaaninfecties.

De dikke zwarte boxen in het flowschema tonen de manieren, onderzocht in dit proefschrift, welke kunnen leiden tot snellere pathogeen detectie en identificatie vanuit subkweek (kolonies op een voedingsbodem), positieve bloedkweken en bloed. Afkortingen; SIRS; systemic inflammatory response syndrome (algehele ontstekingsreactie van het lichaam), NLCR; neutrophil-lymphocyte count ratio, MALDI-TOF MS; matrix assisted laser desorption/ionisation- time of flight mass spectrometry (techniek voor analyse op basis van eiwitspectrum), PCR; polymerase chain reaction (DNA vermeerderingstechniek), AGB; antibiotica gevoeligheidsbepaling. 
zijn, is dit erg tijdrovend. Toekomstige diagnostische testen zouden in staat moeten zijn, om minimaal 5-10 $\mathrm{ml}$ volbloed te verwerken, dit om optimale detectiepercentages te kunnen garanderen. Het aantal pathogenen dat aangetoond kan worden moet worden uitgebreid en volledige AGB zou moeten worden toegevoegd. De verkregen resultaten in dit onderzoek, wijzen tevens uit, dat toekomstige testen zouden moeten kunnen screenen op bepaalde mRNA transcripten. mRNA lijkt een betere marker, om levende pathogenen aan te tonen. Dit in tegenstelling tot DNA of rRNA, welke ook van dode pathogenen afkomstig kunnen zijn. Deze verschillende aspecten zouden idealiter moeten worden gebundeld in 1 simpele point of care test, welke zowel in staat is pathogeen DNA/RNA te detecteren en identificeren en een volledige antibiotica gevoeligheidbepaling uit kan voeren. De test zou gekenmerkt moeten worden door een simpel "monster in, resultaten uit" principe. Resultaten moeten zo snel mogelijk beschikbaar zijn en tevens zouden geen speciale technische vaardigheden noodzakelijk moeten zijn voor het uitvoeren van deze toekomstige test. Momenteel is er nog geen sepsis test commercieel verkrijgbaar, die pathogeen verrijking voor grote volumes volbloed combineert met snelle pathogeen detectie en identificatie. De test die dit ideale scenario het beste benaderd, is de MagicPlex Sepsis Test van Seegene, gecombineerd met pathogeen verrijking met MolYsis (Molzym). Deze combinatie neemt echter ook nog zeker 6 uur in beslag en levert geen volledige identificatie van alle pathogenen. Om deze toekomstige diagnostische test te creëren, zal eerst pathogeen verrijking en pathogeen DNA isolatie uit volbloed moeten worden geoptimaliseerd. De commercieel verkrijgbare MolYsis pathogeen verrijking methode neemt minimaal 2 uur tijd in beslag, afhankelijk van het te verwerken aantal monsters, om pathogeen DNA te verkrijgen uit volbloed. Pathogeen verrijking gecombineerd met DNA isolatie moet volledig geautomatiseerd worden, dit om handelingen en besmettingrisico's te reduceren. Polaris (Biocartis) wordt momenteel volledig geautomatiseerd in een gesloten wegwerp cassette. Wanneer deze cassette beschikbaar wordt gesteld voor onderzoek, zal deze eerst geëvalueerd moeten worden voor $10 \mathrm{ml}$ volbloed, ook in een klinische omgeving.

Het zal erg moeilijk worden om volledige AGB te incorporeren in 1 test, omdat antibiotica resistentie het resultaat is van een groot aantal genen en complexe regulatiemechanismen. Microarray gebaseerde testen zijn al ontwikkeld, om grote aantallen antibiotica resistentie genen te onderzoeken. Het nadeel van microarrays is, dat ze speciale laboratoriumapparatuur vereisen en daarnaast is het vereist, dat technisch vaardige mensen deze testen inzetten. Real-time PCR kan ook ingezet worden, om klinisch relevante antibiotica resistentie genen aan te tonen uit klinische monsters, bijvoorbeeld uit volbloed. Enkele commercieel verkrijgbare sepsis testen bieden al de mogelijkheid, om een klein panel van antibiotica resistentie genen aan te tonen. Voorbeelden zijn VYOO (SIRS-Lab) en MagicPlex Sepsis Test (Seegene). Deze bestaande panels moeten worden uitgebreid, wanneer meer antibiotica resistentie markers en virulentiefactoren beschikbaar komen. Diagnostische testen die een volledige 
AGB uit volbloed bewerkstelligen, zijn nodig om snel een pathogeen specifiek antibioticum toe te kunnen dienen aan een patiënt met een BBI.

Nieuwe ontwikkelingen doen hun intree in de medische wereld en zouden de start kunnen zijn voor snelle pathogeen identificatie gecombineerd met een volledige AGB. Een voorbeeld hiervan is next generation sequencing (NGS). Salipante et al. heeft al aangetoond, dat NGS kan worden gebruikt, om bacteriën te identificeren uit heterogene klinische monsters. Ze gebruikte de diep sequensen technologie op een collectie van sputum monsters van patiënten met cystische fibrose en toonden pathogenen aan in monsters, die negatief waren met de standaard kweekmethoden (hier waren vooral micro-organismen in het spel met complexe voedingseisen). NGS methoden zullen waarschijnlijk snel in staat zijn, om met hoge resolutie genotypes te bepalen en zullen uiteindelijk geïmplementeerd worden in de klinische microbiologie.

De huidige trends in het veld van moleculaire diagnostiek voor infectieziekten, bewegen richting geautomatiseerde high-throughput technologieën, zoals NGS, en zullen enorme hoeveelheden data gaan genereren. Meer mensen met bioinformatica achtergrond en/of ontwikkelde software pakketen, zullen nodig zijn om deze gigantische hoeveelheid aan data te verwerken.

De resultaten gepresenteerd in dit proefschrift laten zien, dat de laboratorium diagnostiek voor BBI significant sneller kan. Moleculaire testen op grote volumes volbloed van BBI patiënten kunnen, wanneer ze geoptimaliseerd zijn, de patiëntenzorg verbeteren en potentieel de morbiditeit en mortaliteit verlagen. Toekomstige prospectieve studies zijn echter nog nodig, om onze bevindingen te bevestigen en om vast te stellen of de volbloed strategie kosteneffectief is. De toekomst zal moeten uitwijzen of gevoelige multiplex PCR testen of NGS, gecombineerd met pathogeen verrijking uit grote volumes bloed, de basis zal gaan vormen voor een simpele point of care test. 


\section{Dankwoord}

Curriculum Vitae

List of Publications 


\section{Dankwoord}

Het einde van deze fase in mijn leven is in zicht en dit zijn de laatste woorden en zinnen, die ik mag schrijven om mijn proefschrift af te ronden. Het is een heftige periode geweest met veel dieptepunten, maar gelukkig ook vele hoogtepunten. Ik heb deze tijd mogen delen met veel mensen die stuk voor stuk belangrijk voor mij zijn geweest, zonder hun was dit proefschrift er waarschijnlijk nooit gekomen.

Allereerst wil ik mijn promotor prof. dr. Cathrien Bruggeman, en copromotoren Adriaan en Petra bedanken voor de goede begeleiding en kritische houding de afgelopen 5 jaar. Ik weet nog goed dat ik voor het eerst met Adriaan van Eindhoven naar Maastricht ben gereisd, om kennis te maken met Cathrien en Petra. Er moest nog een onderzoek opgezet gaan worden en iedereen was nog wat onzeker. Bedankt voor het vertrouwen dat jullie in me hebben gesteld. Ik heb het ervaren als een bijzonder prettige samenwerking. Adriaan, de afgelopen 5 jaar heb jij me vooral onder je vleugels genomen en ik wil jou dan ook in het bijzonder bedanken voor de goede samenwerking. De vele adviezen en motiverende woorden hebben me er doorheen gesleept. Zoals je weet, was het vaak echt een uitdaging om de vele werkplekken en mijn onderwijstaak te combineren met het onderzoek. Ik snap nu eigenlijk nog steeds niet hoe jij al je taken en je grote gezin met elkaar combineert.

Dan natuurlijk een welgemeend woord van dank voor Fontys Hogeschool Toegepaste Natuurwetenschappen, in het bijzonder oud-directeur Mw. dr. Marthie Meester. Beste Marthie, dankzij jou sta ik hier vandaag natuurlijk ook. Je hebt altijd vertrouwen gehad in een goede afloop en me binnen Fontys gesteund daar waar mogelijk. Ook wil ik graag Paul Boelens bedanken, jij hebt me wegwijs gemaakt in het onderwijs en antwoord gegeven op de vele vragen die ik had tijdens mijn eerste jaar als docent. Mijn collega IBIS kantoorgenootjes, Carla, Joost, Yvonne, Wilfried, Vincent, Ömür, Jan, Gertruud en Lilian wil ik bedanken voor de geboden flexibiliteit en het bieden van een luisterend oor. Speciaal voor Jan en Vincent, nog even een Chuck Norris fact: "Chuck Norris's tears could cure all infectious diseases, too bad he never cries". Els, ook jij bedankt voor het invallen als ik weer eens een congres had op mijn onderwijsdag. Gies Evens en Ron Dankers wil ik beide bedanken, omdat ze onmisbaar waren voor mij, als promovenda, binnen Fontys.

Tijdens mijn promotieonderzoek ben ik op verschillende locaties werkzaam geweest. De ene helft van het werk heb ik uitgevoerd op de laboratoria voor Pathologie en Medische Microbiologie van Stichting PAMM, en de andere helft binnen het Jeroen Bosch Ziekenhuis (JBZ). Op alle locaties ben ik hartelijk ontvangen en ik ben dan ook altijd met veel plezier naar het lab gegaan. Bij Stichting PAMM heb ik vooral samengewerkt met arts-microbioloog Arjan Jansz, data-informatiemanager Gerrit Janssen en de laboratorium medewerkers Jitske, Christel, Mariëlle, Marion, en Elna. Ik heb veel van jullie mogen leren. Verder wil ik natuurlijk ook alle andere medewerkers bedanken voor de gezellige tijd. In het bijzonder 
Mark, jij behoorde tenslotte bij de "harde kern" borrelaars. In december 2010 heb ik al mijn "labspullen" verhuist naar Den Bosch, om daar verder te klussen aan mijn onderzoek. Daar ben ik vooral samen gaan werken met moleculair bioloog Mirjam Hermans, artsmicrobioloog Peter Wever, intensive care arts Peter de Jager, klinisch epidemioloog Mirrian Hilbink, arts in opleiding Janna Tosserams en klinisch chemicus Ron Kusters. Dank voor al jullie wetenschappelijke input. Ronald, onze gesprekjes (vaak in de gang), waarin frustraties en blijdschap over artikelen, maar ook andere promotiezaken de revue passeerden, vond ik altijd erg waardevol. Jij hebt alles net achter de rug. Heel veel succes in je verdere carrière. Alle medewerkers van de afdelingen Medische Microbiologie, Moleculaire Diagnostiek en Klinische Chemie verdienen een woord van dank. Jullie hebben dit onderzoek op allerlei fronten ondersteund.

Ook het Catharina ziekenhuis in Eindhoven mag niet onvermeld blijven. Hier zijn belangrijke eerste discussies gevoerd over hoe de diagnostiek voor bloedbaaninfecties eruit zou moeten gaan zien. Herman Kreeftenberg en Arnoud Roos hebben hier een belangrijke rol in gespeeld en ik wil jullie daar dan ook hartelijk voor bedanken. Er is na 5 jaar werk echter nog steeds geen soort van "lakmoes test" beschikbaar. Op de afdeling klinische chemie ben ik ook heel veel geweest en daar hebben Paul van Kaathoven, Jan Michielse en Gitta Stienen altijd voor mij klaargestaan. Bedankt voor de fijne samenwerking.

Ik heb dit promotieonderzoek veelal alleen uitgevoerd. Ik was niet werkzaam in een grote onderzoeksgroep en heb dus veel gehad aan Judith en Wendy, twee collega promovendi van de afdeling Medische Microbiologie van het MUMC. Ik wil jullie graag bedanken voor de leuke samenwerking, jullie mening tijdens werkoverleggen en de gezelligheid op congressen. Dat geldt ook voor René en Suzanne van het Erasmus MC.

Tijdens mijn onderzoek heb ik ook veel samengewerkt met Philips Research en Biocartis. Ik wil Paul vd Wiel, Sigi, Irene, Bart, Roel en Geert hartelijk danken voor de samenwerking en steun. Ook Martine Bos en Paul Savelkoul van het VUMC wil ik bedanken voor de prettige samenwerking en de wetenschappelijke input.

Het vele werk beschreven in dit proefschrift was nooit verzet geweest dankzij de vele enthousiaste stagiaires die ik heb mogen begeleiden. Het begon allemaal met Jeandery, een Antilliaanse schone die bijna 1 jaar lang in dit onderzoek heeft meegedraaid. Jeandery, je hebt mega veel werk verzet en dat heeft ook geleidt tot mooie artikelen, bedankt. Desiree en Wouter konden tegelijk aan de slag op verschillende projecten binnen Stichting PAMM. Jullie hebben ervaren dat het niet altijd gemakkelijk is op meerdere plaatsen werkzaam te zijn. Eenmaal in het JBZ kwam Maurice de boel versterken. Een oude bekende van de PAMM. Maurice, je hebt een start gemaakt met het RNA project. Daarnaast hebben we genoten van je raps. Maikel kon een vliegende start maken binnen het RNA project en daarnaast hebben we veel (om je) gelachen. Ik hoop dat je nu al wat beter kunt koken. Mike was de laatste en heeft ook in 5 maanden veel werk verzet. Ik ben super trots op Jeandery, Maurice, Maikel 
en Mike die hun werk hebben mogen presenteren op het BaMa symposium van de NvMM. Dankzij jullie allemaal is dit boekje zo compleet. Daarnaast ben ik ook blij dat de meeste van jullie een leuke baan hebben gevonden. Bedankt voor de tomeloze inzet en al het harde werken.

Ik heb de afgelopen 5 jaar veel kamergenootjes gehad. Te beginnen met Arantza. Arantza, jij was mijn collega docent/onderzoeker, die ook via Fontys een promotieonderzoek startte. Ondanks het feit, dat we totaal verschillende onderzoeken hadden, konden we elkaar wel steunen qua frustraties, die zijn bij alle promovendi toch veelal gelijk. Daarnaast natuurlijk bedankt voor de gezelligheid bij de etentjes en borrels. Wij vormden met Mark toch de "harde kern" bij de PAMM. Je bent een andere weg ingeslagen en nu bezig als patholoog in opleiding in Leiden. Weer een heel pittig traject en dat doe ik je dus niet na. Dan natuurlijk Lieke, mijn vaste kamergenootje op het JBZ. Ik rond mijn onderzoek iets eerder af dan jij, maar het gaat jou ook zeker lukken. Ik ben blij dat we elkaar zijn tegen gekomen, twee vreemde (een Brabantse en een Limburgse) eendjes in de bijt zeg maar. Bedankt voor al je input aangaande statistische analyses in SPSS, het luisterende oor, maar vooral de gezelligheid. We moeten denk ik wel concluderen dat het ons in het JBZ niet gelukt is een "borrelcultuur" te creëren. Dan wil ik ook de tijdelijke kamergenootjes, dus eigenlijk alle arts-assistenten in opleiding, waarmee ik in Eindhoven of Den Bosch een kamer heb mogen delen: Miranda, Saara, Wouter, An-Emmy, Bas, en Aletta bedanken voor de leuke tijd.

Alle vrienden en kennissen van sportschool BodyLine te Tilburg verdienen een woordje van dank. Ik wil daarbij Bart en Mieke in het bijzonder noemen. Jullie hebben gezorgd voor de broodnodige ontspanning en gezelligheid in de avonduren en weekenden. Natuurlijk moest ik zelf bewegen, maar de motiverende woorden tijdens een van jullie lessen zijn altijd welkom geweest. De aerobic lessen en de vele uren op de spinningfiets hebben me door de drukke tijden heen gesleept. Jan en Noud, bedankt voor de gezelligheid bij het hardlopen in de Oude Warande op zaterdagochtend. Frans, bij onze kennismaking een paar jaar geleden heb ik niet overzien, dat we nu zoveel tijd met elkaar zouden doorbrengen. Je hebt me overgehaald eens mee te gaan fietsen en moet je ons nu eens zien. Je hebt me besmet met het "Van Poppel fietsvirus". Ik hoop dat Bart, ik en jij nog vele mooie tochten mogen maken op de racefiets. Jij staat daarnaast altijd voor ons klaar. Hiervoor ontzettend veel dank.

Mijn lieve vrienden en vriendinnen mogen natuurlijk ook niet ontbreken in dit hoofdstuk van mijn boekje. Enkele van jullie ken ik al zowat mijn hele leven. Ik ben blij dat sommige van jullie ook een promotieonderzoek hebben doorlopen, dan wel er zelf nog mee bezig zijn. Dat praat toch wat makkelijker en creëert begrip voor afwezigheid op feestjes i.v.m. "het werk". Daarnaast hebben de vele feestjes, dinner-dates, en stapavondjes gezorgd voor de nodige ontspanning tijdens de weekenden. Bedankt hiervoor. Linda (dr. Loonen), ondertussen kennen we elkaar al een hele tijd. Samen begonnen aan de HLO in Etten-Leur en een leuke tijd in Vermont (Amerika) beleefd wat onze band alleen maar sterker heeft gemaakt. Geen 
familie maar toch dezelfde achternaam. Ik ben blij dat je mijn paranimf wilt zijn. Steffen, jij nog bedankt voor de mooie foto van het doek welke mijn kaft siert. Het was een minder makkelijke opdracht dan ik dacht.

Lieve pap en mam, ik wil jullie bedanken voor de onvoorwaardelijke steun en jullie vertrouwen in mij. Jullie zijn nog altijd echt geïnteresseerd in mijn dagelijkse bezigheden, inclusief de vele frustraties (al hoop ik wel dat die nu in hoeveelheid gaan afnemen). Mam, ik ben blij dat je zo creatief bent. Dankzij jou heb ik nu een heel bijzondere kaft om mijn boekje zitten. Ik hoop dat je schilder-maatjes nu ook begrijpen wat je geschilderd hebt (ik blijf anders die dochter van dat rare schilderij). Mijn lieve broertje Paul, zusje Lieke, Marcella en Bennie mogen ook niet ontbreken in dit dankwoord. Ik ben blij dat we het altijd zo gezellig kunnen hebben met z'n allen. Lieke, als mijn paranimf heb je een belangrijke rol bij mijn promotie en ik ben blij dat je dit belangrijke moment in mijn leven op deze manier met me wil delen. De rest van mijn familie en schoonfamilie wil ik bedanken voor de interesse in mijn onderzoek. Ik hoop dat na het lezen van dit boekje het allemaal nog duidelijker is geworden.

Dan nog een laatste woord van dank aan de belangrijkste persoon in mijn leven, Bart. We zijn ondertussen al een hele tijd samen en ik heb met jou dus ook bijna alle "ups and downs" in mijn leven gedeeld. Deze bijzondere periode in mijn leven sluit ik nu af en ik ben blij dat jij gedurende dit promotietraject aan mijn zijde hebt gestaan. Je hebt me veel steun gegeven, gemotiveerd en gelukkig ook voor de broodnodige relativering gezorgd. Als registeraccountant sta je toch wat anders tegenover een promotieonderzoek. Nu hebben we hopelijk iets meer tijd om met onze Engelse Bulletjes Mike en Tygo erop uit te gaan in de avonden en weekenden.

Mocht ik toch nog iemand vergeten zijn persoonlijk te noemen, vul dan gerust je naam in op de stippellijn bedankt! 


\section{Curriculum Vitae}

Anne Loonen werd geboren op 18 januari 1983 in Goirle. Na het behalen van het VWO diploma aan het St. Odulphus Lyceum te Tilburg startte zij in 2001 aan de Avans Hogeschool (Etten-Leur en later Breda) met de studie Biologie en Medische Laboratoriumonderzoek. Zij heeft stage gelopen op de Universiteit van Vermont (Burlington, Vermont, USA) onder begeleiding van prof. dr. Nick Heintz en op het Josephine Nefkens Instituut (Rotterdam) onder begeleiding van dr. Ronald de Krijger en dr. Winand Dinjens. Na drie jaar behaalde zij haar diploma en startte ze in 2004 met de Master Medische Biologie aan de Radboud Universiteit te Nijmegen. Hiervoor heeft ze stage gelopen op de afdeling Moleculaire Biologie van het Nijmegen Centre for Molecular Life Sciences (NCMLS) onder begeleiding van dr. Marion Lohrum en op de afdeling Fertiliteit van Organon (Oss) onder begeleiding van Ruud Lagemaat en dr. Sietse Mosselman. In 2007 begon zij aan een promotieonderzoek in het NCMLS, afdeling (cel)fysiologie, onder begeleiding van prof. dr. Peter Deen. Hier deed zij onderzoek naar verstoorde waterhuishouding in het lichaam als gevolg van mutaties in de vasopressine V2 receptor. In 2008 startte zij als docent/onderzoeker bij Fontys Hogeschool Toegepaste Natuurwetenschappen (TNW) te Eindhoven. In samenwerking met het Maastricht Universitair Medische Centrum, Stichting PAMM, en het Jeroen Bosch Ziekenhuis (JBZ) is dit promotieonderzoek tot stand gekomen. Hierbij werd zij begeleid door dr. Adriaan van den Brule (PAMM en later JBZ), prof. dr. Cathrien Bruggeman (MUMC), en dr. ir. Petra Wolffs (MUMC). Naast het uitvoeren van haar promotieonderzoek is zij 2 dagen per week actief geweest als docent op Fontys Hogeschool TNW. In januari 2013 behaalde ze binnen Fontys de basis kwalificatie onderwijs (BKO) en sinds augustus 2013 is ze hier fulltime werkzaam. 


\section{List of publications}

1. Peter M. Burch, Ziqiang Yuan, Anne J. Loonen, and Nicholas H. Heintz, An extracellular signal-regulated kinase 1- and 2-dependent program of chromatin trafficking of c-Fos and Fra-1 is required for cyclin D1 expression during cell cycle re-entry, Molecular and Cellular Biology, 2004.

2. Anne J. Loonen, Nine V. Knoers, Carel H. van Os, and Peter M. Deen, Aquaporin 2 mutations in nephrogenic diabetes insipidus, Seminars in Nephrology, 2008.

3. Esther Korpershoek, Anne J. Loonen, Suzanne Corvers, Francien H. van Nederveen, Jos Jonkers, Xiaoqian Ma, Angelique Ziel-van der Made, Hanneke Korsten, Jan Trapman, Winand N. Dinjens, and Ronald R. de Krijger, Conditional Pten knock-out mice: a model for metastatic phaeochromocytoma, Journal of Pathology, 2009.

4. Anne J. Loonen, Arjan R. Jansz, Herman Kreeftenberg, Cathrien A. Bruggeman, Petra F. Wolffs, and Adriaan J. van den Brule, Acceleration of the direct identification of Staphylococcus aureus versus coagulase-negative staphylococci from blood culture material: a comparison of six bacterial DNA extraction methods, European Journal of Clinical Microbiology and Infectious Diseases, 2010.

5. Anne J. Loonen, Arjan R. Jansz, Jitske Stalpers, Petra F. Wolffs, and Adriaan J. van den Brule, An evaluation of three processing methods and the effect of reduced culture times for faster direct identification of pathogens from BacT/ALERT blood cultures by MALDI-TOF MS, European Journal of Clinical Microbiology and Infectious Diseases, 2011.

6. Anne J. Loonen, Rob Schuurman, and Adriaan J. van den Brule, Highlights from the 7th European meeting on molecular diagnostics, Expert Review of Molecular Diagnostics, 2012.

7. Anne J. Loonen, Arjan R. Jansz, Jeandery N. Bergland, Marion Valkenburg, Petra F. Wolffs, and Adriaan J. van den Brule, Comparative study using phenotypic, genotypic and proteomics methods for identification of staphylococci, Journal of Clinical Microbiology, 2012.

8. Anne J. Loonen, Martine P. Bos, Bart van Meerbergen, Arnold Catsburg, Irene Dobbelaer, Roel Penterman, Geert Maertens, Sigi Neerken, Paul van de Wiel, Paul Savelkoul, and Adriaan J. van den Brule, Comparison of pathogen DNA isolation methods from large volumes of whole blood to improve molecular diagnosis of bloodstream infections, Plos One, 2013.

9. Anne J. Loonen, Arjan R. Jansz, Jeandery N. Bergland, Herman Kreeftenberg, and Adriaan J. van den Brule, Development and clinical evaluation of a tuf gene based real-time PCR for Gram positive cocci: a study using blood cultures and whole blood, manuscript submitted.

10. Anne J. Loonen, Cornelis P. de Jager, Janna Tosserams, Ron Kusters, Mirrian Hilbink, Peter C. Wever, and Adriaan J. van den Brule, Biomarkers and molecular analysis to improve bloodstream infection diagnostics in an emergency care unit, manuscript submitted.

11. Judith Beuving, Anne J. Loonen, Margreet C. Vos, Adriaan J.C. van den Brule, Annelies Verbon, and Petra F.G. Wolffs, Speciation of coagulase-negative staphylococci from positive blood cultures using MALDI-TOF MS: clinical relevance, manuscript submitted.

12. Anne J. Loonen, Petra F. Wolffs, Maurice Habraken, Maikel de Bresser, Mirjam Hermans, and Adriaan J. van den Brule, Tuf mRNA might be a promising marker to detect viable Staphylococcus aureus in blood of bacteremic patients, manuscript in preparation. 
